\title{
1 Cell transcriptomic atlas of the non-human primate Macaca
}

2 fascicularis

4 Lei Han ${ }^{1,2,3,35}$, Xiaoyu Wei ${ }^{1,4,35}$, Chuanyu Liu ${ }^{1,3,35}$, Giacomo Volpe ${ }^{5,35}$, Zhenkun 5 Zhuang ${ }^{1,6,35}$, Xuanxuan Zou ${ }^{1,4,35}$, Zhifeng Wang ${ }^{1,2,35}$, Taotao Pan ${ }^{1}$, Yue Yuan ${ }^{1,4}$, Xiao

6 Zhang $^{7}$, Peng Fan ${ }^{7}$, Pengcheng Guo ${ }^{7}$, Yiwei Lai ${ }^{8}$, Ying Lei ${ }^{1,2,3}$, Xingyuan Liu ${ }^{7}$, Feng $7 \mathrm{Yu}^{8}$, Shuncheng Shangguan ${ }^{9}$, Guangyao Lai ${ }^{9}$, Qiuting Deng ${ }^{1,4}$, Ya Liu ${ }^{1,2}$, Liang Wu ${ }^{1,2,4}$, 8 Quan $\mathrm{Shi}^{1,10}$, Hao $\mathrm{Yu}^{1}$, Yunting Huang ${ }^{1,11}$, Mengnan Cheng ${ }^{1,4}$, Jiangshan $\mathrm{Xu}^{1,4}$, Yang 9 Liu ${ }^{1,4}$, Mingyue Wang ${ }^{1}$, Chunqing Wang ${ }^{1,4}$, Yuanhang Zhang ${ }^{1,4}$, Duo Xie ${ }^{1,4}$, Yunzhi Yang $^{12}$, Yeya Yu ${ }^{12}$, Huiwen Zheng ${ }^{12}$, Yanrong Wei ${ }^{12}$, Fubaoqian Huang ${ }^{1,6}$, Junjie Lei ${ }^{1,4}$, 11 Waidong Huang ${ }^{1,4}$, Zhiyong Zhu ${ }^{1,4}$, Haorong $\mathrm{Lu}^{1,11}$, Bo Wang ${ }^{1,11}$, Xiaofeng Wei ${ }^{1,11}$, 12 Fengzhen Chen ${ }^{1,11}$, Tao Yang ${ }^{1,11}$, Wensi Du ${ }^{1,11}$, Jing Chen ${ }^{1,11}$, Shibo Xu ${ }^{13}$, Juan $\mathrm{An}^{8,14}$, 13 Carl Ward ${ }^{8}$, Zongren Wang ${ }^{15}$, Zhong Pei ${ }^{16}$, Chi-WaiWong ${ }^{17}$, Xiaolei Liu ${ }^{7}$, Huafeng 14 Zhang ${ }^{18}$, Mingyuan Liu ${ }^{7}$, Baoming Qin ${ }^{19}$, Axel Schambach ${ }^{20,21}$, Joan Isern ${ }^{22}$, Liqiang 15 Feng $^{23}$, Yan Liu ${ }^{13}$, Xiangyu Guo ${ }^{24}$, Zhen Liu ${ }^{25}$, Qiang Sun ${ }^{25}$, Patrick H. Maxwell ${ }^{26}$, 16 Nick Barker $^{27}$, Pura Muñoz-Cánoves ${ }^{28}$, Ying Gu${ }^{1}$, Jan Mulder ${ }^{29,30}$, Mathias Uhlen ${ }^{29,30}$, 17 Tao Tan ${ }^{31}$, Shiping Liu ${ }^{1,2,3}$, Huanming Yang ${ }^{1,32}$, Jian Wang ${ }^{1,32}$, Yong Hou ${ }^{1,2,3,12,}$, Xun $18 \mathrm{Xu}^{1,33,12,}$, Miguel A. Esteban ${ }^{7,8,34}$, Longqi Liu ${ }^{1,3,12}$

${ }^{1}$ BGI-ShenZhen, Shenzhen 518103, China.

$21{ }^{2}$ Shenzhen Key Laboratory of Single-Cell Omics, BGI-Shenzhen, Shenzhen 518120, 22 China.

$23{ }^{3}$ Shenzhen Bay Laboratory, Shenzhen 518000, China.

$24{ }^{4}$ College of Life Sciences, University of Chinese Academy of Sciences, Beijing 100049, 25 China.

$26{ }^{5}$ Hematology and Cell Therapy Unit, IRCCS-Istituto Tumori 'Giovanni Paolo II', Bari 27 70124, Italy.

$28{ }^{6}$ School of Biology and Biological Engineering, South China University of Technology, 
Guangzhou 510006, China.

${ }^{7}$ State Key Laboratory for Zoonotic Diseases, Key Laboratory for Zoonosis Research of Ministry of Education, Institute of Zoonosis, College of Veterinary Medicine, Jilin University, Changchun 130062, China.

${ }^{8}$ Laboratory of Integrative Biology, Guangzhou Institutes of Biomedicine and Health, Chinese Academy of Sciences, Guangzhou 510530, China.

${ }^{9}$ Joint School of Life Sciences, Guangzhou Institutes of Biomedicine and Health and Guangzhou Medical University, Guangzhou 510530, China.

${ }^{10}$ Department of Biology, University of Copenhagen, Copenhagen DK-2200, Denmark. ${ }^{11}$ China National GeneBank, BGI-Shenzhen, Shenzhen 518120, China.

${ }^{12}$ BGI College and Henan Institute of Medical and Pharmaceutical Sciences, Zhengzhou University, Zhengzhou 450000, China.

${ }^{13}$ Institute for Stem Cells and Neural Regeneration, School of Pharmacy, State Key Laboratory of Reproductive Medicine, Nanjing Medical University, Nanjing 211166, China.

${ }^{14}$ University of Science and Technology of China, Hefei 230026, China.

${ }^{15}$ Department of Urology, First Affiliated Hospital, Sun Yat-sen University, Guangzhou 510000, China.

${ }^{16}$ Department of Neurology, First Affiliated Hospital, Sun Yat-sen University, Guangzhou 510000, China.

${ }^{17}$ Huazhen Biosciences, Guangzhou 510900, China.

${ }^{18}$ Department of Orthopedics, Tianjin Medical University General Hospital, Tianjin 300052, China.

${ }^{19}$ Laboratory of Metabolism and Cell Fate, Guangzhou Institutes of Biomedicine and Health, Chinese Academy of Sciences, Guangzhou 510530, China.

$4{ }^{20}$ Institute of Experimental Hematology, Hannover Medical School, Hannover 30625, Germany. 
${ }^{21}$ Division of Hematology/Oncology, Harvard Medical School, Boston MA 02115, USA.

${ }^{22}$ Spanish National Center on Cardiovascular Research (CNIC), Madrid E-28029, Spain. ${ }^{23}$ State Key Laboratory of Respiratory Diseases, Guangzhou Institutes of Biomedicine and Health, Chinese Academy of Sciences, Guangzhou 510530, China.

${ }^{24}$ Jinan University, Guangzhou 510632, China.

${ }^{25}$ Institute of Neuroscience, State Key Laboratory of Neuroscience, CAS Key Laboratory of Primate Neurobiology, CAS Center for Excellence in Brain Science and Intelligence Technology, Chinese Academy of Sciences, Shanghai 200031, China.

${ }^{26}$ Cambridge Institute for Medical Research, Department of Medicine, University of Cambridge, Cambridge CB2 0XY, United Kingdom.

${ }^{27} \mathrm{~A}$ *STAR Institute of Molecular and Cell Biology, Singapore 138648, Singapore.

${ }^{28}$ Department of Experimental and Health Sciences, Pompeu Fabra University (UPF), ICREA and CIBERNED, Barcelona E-08003, Spain.

${ }^{29}$ Department of Protein Science, Science for Life Laboratory, KTH-Royal Institute of Technology, Stockholm 17121, Sweden.

${ }^{30}$ Department of Neuroscience, Karolinska Institute, Stockholm 17177, Sweden.

${ }^{31}$ State Key Laboratory of Primate Biomedical Research, Institute of Primate Translational Medicine, Kunming University of Science and Technology, Kunming 650500, China.

${ }^{32}$ James D. Watson Institute of Genome Sciences, Hangzhou 310058, China.

${ }^{33}$ Guangdong Provincial Key Laboratory of Genome Read and Write, Shenzhen 518120 , China.

${ }^{34}$ Institute of Stem Cells and Regeneration, Chinese Academy of Sciences, Beijing 100101, China.

${ }^{35}$ These authors contributed equally to this work.

Corresponding authors. Email: houyong@genomics.cn (Y.H.), xuxun@genomics.cn (X.X.), miguel@gibh.ac.cn (M.A.E.), liulongqi@genomics.cn (L.L.). 
85 Studying tissue composition and function in non-human primates (NHP) is crucial to

86 understand the nature of our own species. Here, we present a large-scale single-cell and

87 single-nucleus transcriptomic atlas encompassing over one million cells from 43 tissues

88 from the adult NHP Macaca fascicularis. This dataset provides a vast, carefully

89 annotated, resource to study a species phylogenetically close to humans. As proof of

90 principle, we have reconstructed the cell-cell interaction networks driving Wnt

91 signalling across the body, mapped the distribution of receptors and co-receptors for

92 viruses causing human infectious diseases and intersected our data with human genetic

93 disease orthologous coordinates to identify both expected and unexpected associations.

94 Our Macaca fascicularis cell atlas constitutes an essential reference for future single-

95 cell studies in human and NHP.

96

97

98

99

100

101

102

103

104

105

106

107

108

109

110

111

112 


\section{MAIN TEXT}

115 Global initiatives such as the Human Cell Atlas are aiming to chart the cell types and

116 cell states of all tissues in the human body using high-throughput single-cell/nucleus

117 RNA-sequencing (sc/snRNA-seq) and other technologies ${ }^{1-5}$. The ultimate goal of these

118 efforts is to create complete reference maps across different ethnic groups, ages, 119 environmental conditions and pathologies. A major obstacle in this endeavour is that 120 accessing a wide range of 'high quality' human samples and obtaining enough sample 121 size is complicated by relevant practical and ethical considerations. Model animals (e.g., 122 mouse and rat) are a useful resource to fill knowledge gaps ${ }^{6-8}$, in particular the effects 123 of experimental perturbation, but due to profound phylogenetic differences many 124 developmental, physiological and pathological aspects are not mimicked in humans. 125 Given the evolutionary proximity, NHP present an excellent alternative (the nearest-to126 human) when no other suitable models exist. Generating a NHP cell atlas will produce 127 an extensive catalogue of human disease and age-related features that can be modelled 128 in NHP. It will also provide unique insights into the evolutionary and adaptative 129 mechanisms underlying changes in body function between the two species. In this 130 regard, it could for example discover tissue regenerative capacities selectively 131 maintained in NHP and potential ways to boost them in human.

132 NHP encompass a large and very diverse group of species with major ecological, 133 dietary, locomotor and behavioural differences ${ }^{9-11}$. Because of their close evolutionary 134 proximity to humans among NHP, overall characteristics and wider availability, 135 macaques are primarily employed for research purposes worldwide including human 136 disease modelling and preclinical safety assessment studies ${ }^{12,13}$. Here, we have used 137 adult Macaca fascicularis (cynomolgus monkey) to generate the largest single-cell 138 transcriptomic NHP dataset to date, encompassing over 1 million individual cells/nuclei 139 from 43 tissues covering all major systems (nervous, immune, endocrine, 140 cardiovascular, respiratory, digestive, skeletal, reproductive and urinary), all performed 
141 with the same droplet-based approach ${ }^{14}$. To facilitate the exploration of this dataset, we

142 have created the first version of the Non-Human Primate Cell Atlas or NHPCA, an open

143 and interactive database (https://db.cngb.org/nhpca/) that will be regularly updated with

144 subsequent sc/snRNA-seq Macaca fascicularis datasets focused on development, aging,

145 disease and drug responses, as well as other omics datasets and data from other NHP

146 species.

\section{Generation of an adult monkey single-cell transcriptomic atlas}

149 We isolated cells/nuclei from 43 different tissue samples from three male and three 150 female six-year-old Macaca fascicularis monkeys (Fig. 1a and Supplementary Table

151 1a). Bladder (two), cerebellum (two), diaphragm (two), gallbladder (two), kidney (two),

152 liver (three), lung (two), salivary gland (two), subcutaneous (two) and visceral adipose

153 tissue (two) were analyzed as biological replicates to assess individual and gender

154 variability, observing good overlap in all cases (Extended Data Fig. 1). Most of the

155 tissues were profiled by snRNA-seq ${ }^{15-17}$, which allows both to circumvent

156 complications associated with stressful dissociation protocols that can alter the cell

157 transcriptome and to profile cells from frozen tissues for removing the need of sample

158 processing immediately after tissue acquisition. However, due to technical limitations

159 in obtaining high quality nuclei, scRNA-seq was performed for colon, duodenum,

160 spleen, stomach, lymph node and bone marrow. Peripheral blood mononuclear cells

161 (PBMC) were also profiled using scRNA-seq. All experiments used the DNBelab C4

162 droplet-based platform for library generation ${ }^{14}$. To ensure quality, all cells with a gene

163 count lower than 500 and/or mitochondrial content higher than $10 \%$ were excluded. We

164 also applied DoubletFinder to detect and remove doublets, which accounted for roughly

$1655 \%$ of the estimated total cell/nuclei. Overall, we retained transcriptomic data for a total

166 of 1,084,164 cells/nuclei (Fig. 1a), with numbers ranging from 99,123 in the cerebellum

167 to 2,039 in the duodenum (Supplementary Table 1a). Global visualization of cell

168 clustering using Uniform Manifold Approximation and Projection (UMAP) showed 
169 that each tissue clusters separately, with tissues from the same system generally

170 clustering closer (Fig. 1a, b and Extended Data Fig. 2-6). We then performed

171 individual UMAP representations for each tissue and applied unbiased graph-based

172 Seurat clustering, which identified 463 cell clusters among all tissues (Extended Data

173 Fig. 7-10). Based on the expression levels of cell type-specific markers (Extended

174 Data Fig. 11), we identified 106 cell types in the global UMAP view of all tissues (Fig.

175 1c and Supplementary Table 1b, c). These were roughly categorized into epithelial

176 cells (40 clusters), immune cells (13 clusters), endocrine cells ( 11 clusters), muscle cells

177 (9 clusters), stromal cells ( 7 clusters), endothelial cells ( 7 clusters), neurons ( 7 clusters),

178 glia (7 clusters), mesothelial cells (3 clusters), adipocytes (1 cluster) and unknown cells

179 (1 cluster from carotid). On average, we detected 1,368 genes and 3,024 unique

180 molecular identifiers (UMI) per cell. The median gene count per tissue varied between

1813,016 in the neocortex and 736 in the case of PBMC, while UMI ranged between 8,015

182 for the neocortex and 1,313 for the prostate (Extended Data Fig. 12). The number of

183 cells for each of these 106 cell types ranged from 87,890 granule cells in the cerebellum

184 to 37 bone marrow stromal cells (Extended Data Fig. 13). Reassuringly, many of the

185106 clusters were largely composed of a cell type belonging to a specific tissue, such

186 as cerebellar granule cells in cluster 45 , hepatocytes in clusters 87 and 88 , epididymis

187 stereociliated cells in cluster 29 and salivary acinar cells in cluster 83 (Fig. 1c and

188 Extended Data Fig. 14a). However, cell types such as endothelial, stromal and various

189 immune cells were shared between different tissues, as expected (Extended Data Fig.

190 14b). A detailed annotation of all cell populations detected in every tissue is provided

191 in Extended Data Figure 7-10 and Supplementary Table 1d, e. Our Macaca

192 fascicularis atlas is the largest NHP single-cell transcriptome dataset to date and can be

193 explored interactively by tissue, cell type and gene through our NHPCA database.

195 Common cell types across monkey tissues 
196 We inspected whether common cell types distributed throughout different tissues in the

197 monkey body display tissue-specific transcriptional programs $s^{3,18-20}$. First, we

198 selectively clustered stromal cells, macrophages (including microglia), endothelial cells

199 and smooth muscle cells from all sequenced tissues. While observing a considerable

200 diversity, many cell clusters grouped together on the basis of tissue origin, such as

201 stromal cells from the female reproductive system, microglia from the central nervous

202 system, endothelial cells from the respiratory system and smooth muscle cells from the

203 male reproductive system (Extended Data Fig. 15a-d). We also performed

204 differentially expressed gene (DEG) analysis to obtain tissue-specific signatures,

205 revealing a substantial heterogeneity among these common cell types across all tissues

206 (Extended Data Fig. 15e-h and Supplementary Table 2a-d).

207 Our transcriptomic profiling of single nuclei offers the possibility of studying cell 208 populations that cannot be characterized by conventional scRNA-seq analysis, such as 209 myonuclei from multinucleated skeletal muscle fibers. We grouped and re-clustered 210 cells from tissues in our atlas known to contain skeletal muscle cells (diaphragm, tongue, 211 esophagus and abdominal wall). This showed two distant populations in abdominal wall 212 and diaphragm, whereas nuclei from esophagus and tongue where more concentrated 213 (Fig. 2a). The separation of nuclei in abdominal wall and diaphragm corresponded to $214 \mathrm{MYH}^{+}$type I (slow-twitch) and $\mathrm{MYH}^{+}$type II (fast-twitch) myofibers ${ }^{21}$ (Fig. 2b, c 215 and Supplementary Table 2e-g). In contrast, type I and type II tongue myonuclei were 216 in close vicinity, which may be related to the tongue being a highly innervated muscle ${ }^{22}$.

217 Differential threshold of $M Y H 2$ and GPD2 further subdivided type II myonuclei into 218 type IIa $\left(M Y H 2^{\text {high }}\right)$ and type IIb $\left(M Y H 2^{\text {low }} G P D 2^{+}\right)$. In addition, we discriminated, 219 albeit at low proportions, $N A V 3^{+}$neuromuscular junction (NMJ) nuclei in the 220 diaphragm and ETV $^{+}$myotendinous junction (MTJ) nuclei in both tongue and 221 diaphragm (Fig. 2b-d). Moreover, we detected $P A X 7^{+}$nuclei from satellite cells (the 222 stem cells from the skeletal muscle lineage), and a small cluster of $L V R N^{+}$ 223 fibroadipogenic progenitors (FAP) could be annotated in the diaphragm. Skeletal 
224 muscle nuclei displayed subtype-specific and tissue-specific gene expression signatures 225 and gene ontology (GO) terms (Fig. 2e, f and Extended Data Fig 16a-c). We also 226 noticed substantial myonuclei heterogeneity within the same subtype and tissue (Fig. 227 2f).

228 Next, to explore the heterogeneity between different types of adipocytes, we 229 grouped and re-clustered cells from subcutaneous and visceral adipose tissues, resulting 230 in 10 major clusters (Extended Data Fig. 17a). We observed a marked distinction 231 between mature adipocytes and adipocyte progenitors, as reflected by the differential 232 expression of $A D I P O Q$ and $C D 34$ (Extended Data Fig. 17b). Visceral mature 233 adipocytes and adipocyte progenitors displayed enriched expression of ITLN1, in 234 agreement with visceral adipocytes having mesothelial origin ${ }^{23}$, and also high 235 mitochondrial activity exemplified by high expression of ND4, ATP6 and COX3 ${ }^{24,25}$ 236 (Extended Data Fig. 17c, d). In contrast, subcutaneous mature adipocytes and 237 adipocyte progenitors were enriched in FOS. Likewise, SLC11A1 and SPOCK3 marked 238 mature subcutaneous and visceral adipocytes, respectively. Adipocyte progenitors 239 contained two populations for visceral tissue $\left(W T 1^{+}\right.$and $\left.C F D^{\text {high }}\right)$, three for 240 subcutaneous tissue $\left(E S R 1^{+}, C X C L 14^{+} A P O D^{+}\right.$and $\left.D P P 4^{+}\right)$and one shared between 241 both tissues $\left(\mathrm{NOX}^{+}\right)($Extended Data Fig. 17a, c and d). Within the subcutaneous $242 C X C L 14^{+} A P O D^{+}$progenitor cluster, we observed a population of $C F D^{\text {high }}$ cells that also 243 co-expressed DPP4, a marker of highly proliferative adipocyte progenitors in both 244 mouse and human ${ }^{26}$. However, we did not detect significant proliferation in any of the 245 monkey adipocyte progenitor populations based on the expression of the pan-cycling

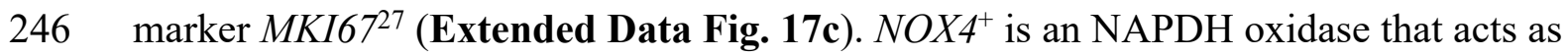
247 a switch from insulin-induced proliferation to adipocyte differentiation, suggesting that 248 the shared cluster is a converging route for both adipose tissues towards adipocytic 249 maturation $^{28}$.

250 Finally, we grouped and re-clustered all tissues that contain mesothelial cells, a type 251 of specialized epithelial cells. Mesothelial cells from bladder, ovary and fallopian tube 
252

were in close proximity while those from other tissues clustered more separately (Fig. 2g). We also observed intra-tissue heterogeneity, in particular for visceral adipose tissue and ovary. In the former, we observed a cluster of immune-like mesothelial cells that, aside from the expression of the typical mesothelial markers (MSLN, ITLN1 and PKHD1L1), express high levels of immune cell markers (e.g., PTPRC, IL 7R and TRAC)

(Fig. 2h). This is in agreement with the emerging concept that structural cells display immune cell properties ${ }^{3,18}$ and the known immunomodulatory role of visceral adipose tissue in responses to bacteria in the gut ${ }^{29}$. Interestingly, in the ovary, we identified a classical mesothelial population and two close $P A X 8^{+}$epithelial-like populations (one mature and one progenitor-like) of mesothelial origin ${ }^{30}$ (Fig. 2i-k). Progenitor-like ovarian epithelial cells expressed well-known stem cell markers such as LGR5, $M E C O M$ and $C D 44^{31}$.

These findings add up to the growing understanding of common cell type heterogeneity and tissue-specific molecular signatures ${ }^{3,18-20}$. Our data provide a new resource for further dissecting these differences, clarifying the underlying mechanisms and studying interspecies differences ${ }^{32}$.

\section{Analysis of Wnt signaling components identifies potential stem cell populations}

A single-cell body atlas of large dimensions like ours is ideal for the systematic investigation of multifaceted cell-cell interactions including those occurring in cytokine or growth factor-mediated signaling pathways such as the Wnt (wingless-related integration site) pathway ${ }^{33,34}$. Besides playing essential roles in embryonic development, Wnt factors control growth and maintenance of numerous tissues throughout life. Consistently, Wnt signaling effects are associated with the regulation of adult stem cell function ${ }^{35}$. To exert this role, Wnt factors bind to specific receptors (FZD, frizzled) and co-receptors (LRP, low-density lipoprotein receptor related protein). In addition, LGR (leucine rich repeat containing G protein-coupled receptor) proteins (LGR4, 5 and 6 ) act as amplifiers of Wnt signals by inhibiting negative regulators ${ }^{36}$. Accordingly, 
280 LGR5 and 6 often mark and regulate adult homeostatic and facultative stem cells, 281 mostly of epithelial origin, in multiple mammalian tissues, whereas LGR4 has a 282 widespread distribution and less clear function. We thus performed a survey of LGR 283 proteins throughout the monkey body to thoroughly dissect cells targeted by the Wnt 284 pathway and identify previously unappreciated stem cell populations. In this regard, it 285 is worth noting that the majority of reports of LGR5-expressing cells to date have been 286 performed with genetically engineered mouse models due to the lack of specific tools 287 and reagents to study other mammals ${ }^{36}$.

288 LGR5 was detected across several monkey tissues, unexpectedly with the highest 289 expression in type I skeletal muscle myonuclei, epithelial cells of the uterus and 290 fallopian tube, oligodendrocyte progenitor cells (OPC) and renal distal convoluted 291 tubule cells (DCTC) (Fig. 3a). With the exception of epithelial cells in the uterus and 292 fallopian tube ${ }^{36}$, these tissues have not previously been reported to contain $\mathrm{LGR}^{+}$cells 293 in mammalian adulthood. The expression of LGR6 appeared to be more restricted 294 (Extended Data Fig. 18a), with higher abundance in cardiomyocytes, thyroid follicular 295 cells, folliculostellate cells of the pituitary gland and the previously reported smooth 296 muscle cells ${ }^{37}$ (Extended Data Fig. 19-22). We also detected $L G R 5^{+}$or $L G R 6^{+}$cells in 297 selected cell populations of numerous other tissues including both previously reported 298 (e.g., ovary epithelial cells ${ }^{31}$, hepatocytes ${ }^{38}$ and colon enterocytes ${ }^{39}$ ) and unreported 299 (e.g., $L G R 5^{+}$cells in bipolar cells of the retina ${ }^{40}$ ) (Fig. 3a, Extended Data Fig. 18a and 300 19-22). In general, $L G R 5$ and $L G R 6$ did not overlap, apart from fallopian tube epithelial 301 cells and vagina smooth muscle cells (Extended Data Fig. 18b). Moreover, we 302 observed little overlap between $L G R 5^{+}$or $L G R 6^{+}$cells with those expressing MKI67, 303 apart from epithelial cells of the fallopian tube and uterus and basal cells of the salivary 304 gland (Extended Data Fig. 19-22 and Supplementary Table 3a-c). In contrast to $305 L G R 5$ and 6, LGR4 was ubiquitously expressed across most tissues, with the highest 306 expression in pancreatic acinar, beta and ductal cells, Müller cells of the retina and 307 adipocytes (Extended Data Fig. 18c). 
In the kidney, $L G R 5^{+}$cells were mostly enriched in the DCTC and to a lesser extent

309 in the descending and ascending loop of Henle (Fig. 3a and Extended Data Fig. 20).

310 To support this observation, we performed single-cell Assay for Transposase

311 Accessible Chromatin sequencing (scATAC-seq) of monkey kidney and integrated the

312 results with our kidney snRNA-seq data dataset $(\mathrm{N}=6,879)$ (Fig. 3b, c and Extended

313 Data Fig. 23a, b). The analysis showed peaks of open chromatin at the $L G R 5$ promoter

314 and a putative enhancer open in the same cell types expressing LGR5 (Fig. 3d). As

315 validation, we performed single-molecule fluorescence in-situ hybridization (smFISH)

316 for LGR5, which showed strong expression in selected kidney tubules (Fig. 3e).

317 Moreover, GO analysis of DEG comparing the $L G R 5^{+}$fractions of DCTC, ascending

318 and descending loop of Henle revealed the enrichment of pathways involved in kidney

319 development in DCTC (Fig. 3f), suggesting the possibility that these are progenitor

320 cells. This was strengthened by the observation that DCTC $L G R 5^{+}$cells co-express

321 renal progenitor cell markers such as PAX2, LHX1 and TNFRSF1941,42. We also

322 integrated our data with available human ${ }^{43}$ and mouse ${ }^{44}$ kidney snRNA-seq datasets.

323 Despite observing good integration, we noticed very little, or no, LGR5 expression in

324 those adult human or mouse kidney datasets ${ }^{45}$ (Extended Data Fig. 24a-c).

325 In the neocortex, integration of available human ${ }^{46}$ and our own mouse snRNA-seq

326 data with our monkey data pointed as well at differential LGR5 expression patterns

327 between species. LGR5 expression was highest in OPC in monkey and in

328 oligodendrocytes in human, whereas in mouse it was higher in inhibitory neurons than

329 OPC and oligodendrocytes (Extended Data Fig. 25a-c). Pseudotime ordered by

330 Monocle 2 of the OPC maturation trajectory towards oligodendrocyte showed

331 concentration of $L G R 5$ in monkey OPC (Extended Data Fig. 25d, e). Likewise, double

332 immunofluorescence for the OPC marker PDGFRA and LGR5 confirmed their co-

333 expression in OPC from monkey neocortex (Extended Data Fig. 25f). The observation

334 that type I skeletal myonuclei and cardiomyocytes ranked first in expression of $L G R 5$

335 and $L G R 6$ in monkey tissues, respectively, was intriguing (Fig. 3a and Extended Data 
336 Fig. 18a). To inspect this further, we grouped and re-clustered all types of muscle cells 337 (skeletal, smooth and cardiac) in our atlas (Fig. 3g). $L G R 5$ was more enriched in $M Y H 7^{+}$ 338 slow-twitch myonuclei of the abdominal wall and diaphragm (Fig. 3h), whereas LGR6 339 was higher in cardiomyocytes and smooth muscle cells (aorta, ovary, carotid and vagina) 340 (Extended Data Fig. 26a). LGR5 and LGR6 expression in slow-twitch skeletal 341 myonuclei and in cardiomyocytes, respectively, were validated by smFISH (Fig. 3i and 342 Extended Data Fig. 26b). In mouse, LGR5 is known to be expressed in NMJ 343 myonuclei ${ }^{47}$ and a subset of satellite cells activated upon injury ${ }^{48}$, but we did not detect 344 LGR5 enrichment in either cell type in our monkey dataset (Extended Data Fig. 19). 345 The lack of LGR5 enrichment in monkey satellite cells is unsurprising given that we 346 did not apply any injury to the skeletal muscle tissues profiled. Yet, we could detect 347 LGR6 in cardiomyocytes using previously reported mouse and human snRNA-seq 348 datasets $^{49,50}$ (Extended Data Fig. 26c, d). Similarly, LGR6 was enriched in several 349 monkey pituitary cell populations, being most highly expressed in folliculostellate cells, 350 which have been reported to be pituitary gland stem cells ${ }^{51}$ (Extended Data Fig. 26e). 351 Consistently, those cells also expressed other progenitor markers such as SOX2, PAX6, $352 C D 44$ and $C X C R 4$ (Extended Data Fig. 26f). Moreover, GO analysis of DEG specific 353 to this $L G R 5^{+}$population compared to other pituitary cells showed enrichment of terms 354 related to development (Extended Data Fig. 26g).

355 Next, we profiled the genes encoding Wnt factors and the R-spondin family 356 (RSPO1-4) of ligands for LGR proteins ${ }^{35,36}$ in a panel of monkey tissues containing 357 cells with high LGR5 (kidney, epididymis, fallopian tube, liver, ovary, neocortex and 358 diaphragm) and LGR6 (heart and pituitary gland) expression (Extended Data Fig. 27a, 359 b and 28-31). This allowed us to dissect the potential cell-cell interaction networks 360 driving Wnt signalling throughout the monkey body. Notably, RSPO cytokines were 361 widely distributed but displayed higher expression in mesenchymal-like cells (e.g., 362 smooth muscle cells of epididymis, hepatic stellate cells and folliculostellate cells of 363 the pituitary gland) and mesothelial cells (e.g., of diaphragm, fallopian tube and ovary) 
364 of different tissues. Interestingly, $\mathrm{RSPO} 2$ was also high in inhibitory neurons of the 365 neocortex (Extended Data Fig. 30). The expression of Wnt factors was more limited 366 and in general lower than RSPO cytokines but we noticed high levels of WNT9B in 367 principal cells of the collecting duct in kidney (Extended Data Fig. 27a, c), WNT2B in 368 mesothelial cells of the fallopian tube (Extended Data Fig. 29a) and ovary (Extended 369 Data Fig. 30c), and as expected WNT2 in endothelial cells of the $\operatorname{liver}^{52}$ (Extended 370 Data Fig. 29c). Wnt9b is an essential regulator of kidney embryonic development in 371 multiple species and of kidney regeneration in lower vertebrates ${ }^{53}$. Supporting the 372 snRNA-seq data, scATAC-seq analysis of the WNT9B locus revealed increased 373 enhancer accessibility in monkey principal cells compared to other kidney cell types 374 (Extended Data Fig. 27d). In contrast, we detected low WNT9B expression in available 375 mouse $^{44}$ and human ${ }^{46}$ snRNA-seq datasets (Extended Data Fig. 27e). WNT9B may be 376 responsible for inducing LGR5 (a Wnt pathway target) in a fraction of DCTC, 377 potentially creating a feedback loop that amplifies WNT9B signals to keep those cells 378 in a progenitor state. In fact, Wnt factors are known to act predominantly on 379 neighbouring cells ${ }^{33,35}$, and cells of the collecting duct and DCTC are in closer 380 proximity than other nephron structures (Extended Data Fig. 27f). We further included 381 Wnt receptors and other co-receptors ${ }^{54}$ in the analysis, and also the TCF family of 382 transcription factors bound by $\beta$-catenin ${ }^{55}$, as a resource for additional exploration in 383 these tissues (Extended Data Fig. 27a,b and 28-31).

384 Therefore, we have reconstructed the Wnt signaling network in monkey tissues and 385 identified cell types with potential progenitor or homeostatic characteristics. Additional 386 signaling pathways and/or ligand-receptor interactions can be explored through our 387 NHPCA database.

Prediction of viral infection vulnerability in monkey tissues

390 To demonstrate the utility of our atlas for advancing the knowledge of disease 391 pathogenesis, we first mapped the expression of the main viral receptors/co-receptors 
392 for a panel of 126 viruses including respiratory ones across all monkey tissues. As

393 expected, NCAM1 (cytomegalovirus receptor) was enriched in astrocytes,

394 oligodendrocytes and neurons, consistent with the knowledge of this virus attacking the 395 central nervous system ${ }^{56}$. In contrast, $C D 46^{57}$ (receptor for Measles and Herpes viruses)

396 was enriched in epithelial cells from bladder, female and male reproductive system, and 397 liver endothelial cells (Fig. 4a, Extended Data Fig. 32 and Supplementary Table 4a). 398 Given the emergency state of the current COVID-19 pandemic caused by SARS-CoV$3992^{58}$, we focused on its receptor ACE2 and co-receptor TMPRSS2 $2^{59}$ to assess how 400 widespread and homogeneous their expression is in monkey tissues. This offers the 401 major advantage of studying COVID-19 pathogenesis in a species phylogenetically 402 close to humans ${ }^{60}$, and also provides the possibility of profiling cell types and/or tissues 403 that have not been studied in human. In this regard, although the lung is the 404 predominantly affected tissue in COVID-19, it is important to clarify what other tissues 405 are targeted to better understand the disease course and its transmissibility ${ }^{61}$. TMPRSS2 406 displayed a broad expression across multiple monkey tissues, whereas $A C E 2$ had a 407 more restricted pattern. The highest ACE2 expression was found in epithelial cells from 408 gallbladder (glandular cells), kidney (mostly proximal tubule cells), lung (ciliated, club 409 and alveolar type 2 [AT2] cells) and liver (hepatocytes and cholangiocytes) (Fig. 4b, 410 Extended Data Fig. 33, 34 and Supplementary Table 4b). ACE2 in these tissues was 411 remarkably heterogeneous, suggesting that regulatory mechanisms fine-tune its 412 expression levels. Notably, double positive $\left(A C E 2^{+} T M P R S S 2^{+}\right)$cells have a higher risk 413 of infection by SARS-CoV-2 $2^{59}$ but it remains unclear what tissues and cell types 414 throughout the human body co-express these genes. We noticed the largest overlap 415 between ACE2 and TMPRSS2 in monkey gallbladder cells in agreement with reports of 416 COVID-19 patients developing acute cholecystitis ${ }^{62}$. Significant co-expression was 417 also observed in ciliated and club cells of the lung, as expected ${ }^{63,64}$, and, interestingly, 418 proximal and connecting tubule cells of the kidney. A smaller overlap was observed in 419 hepatocytes, bladder epithelial cells and pancreatic beta and ductal cells (Fig. 4c). Next, 
we performed a comparative analysis of $A C E 2$ and TMPRSS2 distribution in human $3,6,43$ and monkey. A similar distribution was seen in both the gallbladder and liver in the two species, while distinct patterns were observed for proximal tubule cells of the kidney and for ciliated and AT2 cells of the lung (Extended Data Fig. 35a). This is important because it implys a mechanism by which the infection with SARS-CoV-2 in the two species could have different consequences.

As a representative tissue with high but heterogeneous $A C E 2$ expression and a significant proportion of $A C E 2^{+} T M P R S S 2^{+}$cells, we studied the kidney in more detail by looking at the integration of snRNA-seq and scATAC-seq data. Analysis of open chromatin regions revealed discrete peaks in the ACE2 locus with the highest signal detected in a population of proximal tubule cells that also contains the highest proportion of $A C E 2$-expressing cells (Fig. 4d). Motif analysis demonstrated that $A C E 2$ promoter and enhancer regions are enriched in STAT1 and 3, FOXA1, JUNB and several $I R F$ (interferon response factor) binding sites (Fig. 4e). These transcription factors have important immune functions and are targets of tissue protective and innate immune responses such as those mediated by interleukin-6 (IL6), interleukin-1 (IL1) and interferons ${ }^{65}$. In this regard, dysregulation of both IL6 and IL1 $\beta$ has been implicated in the pathogenesis of severe COVID-1966. Thus, we investigated the co-expression of their receptors $(I L 6 R, I L 1 R 1$ and ILIRAP) with ACE2 in monkey kidney, only observing good correlation with $A C E 2$ in proximal tubule cells for IL6R (Extended Data Fig. 35b). These observations imply a potential link between IL6, STAT transcription factors and enhanced $A C E 2$ expression in specific tissues such as the kidney that can either facilitate the existence of viral reservoirs or exacerbate COVID19 disease progression due to increased viral dissemination (Extended Data Fig. 35c). In addition to ACE2 and TMPRSS2, numerous other molecules have been implicated in facilitating SARS-CoV-2 binding to the cell surface or in COVID-19 pathogenesis $^{67,68}$. Their expression or co-expression in monkey tissues, as well as other 
447 potential associations and other virus-host interactions can be explored using our

448 NHPCA database.

\section{Investigation of common human traits and genetic diseases in monkey}

451 We next assessed the effect of genetic variation linked to complex human traits and 452 diseases by applying Genome Wide Association Studies (GWAS) to our monkey 453 dataset. We linked human single-nucleotide polymorphisms from 163 GWAS taken 454 from the UK Biobank to orthologous coordinates in the monkey single-cell 455 transcriptome to calculate the enrichment of traits across the genes expressed in each 456 cell cluster annotated in our dataset. As a general trend, we observed enriched 457 heritability for neurological traits such as 'schizophrenia', 'depression' or 'autism' in 458 clusters corresponding to neuronal and glial cells (Fig. 5a, Extended Data Fig. 36 and 459 Supplementary Table 5a). Similarly, we observed enrichment of Alzheimer's disease 460 traits in immune cells, in line with the knowledge that immune dysfunction contributes 461 to the pathogenesis of this disease ${ }^{69}$. Consistent with expectations, we also noticed 462 enrichment of immunological-related traits ('lymphocyte count', 'monocyte count' and 463 traits related to immune disorders) in myeloid cells and B and T lymphocytes. Likewise, 464 blood related traits such as 'mean sphered cell volume' and 'red blood cell distribution 465 width' were enriched in erythrocytes and bone marrow progenitor cells. Interestingly, 466 however, we observed some unexpected trends for traits like 'body mass index' or 467 'waste ratio'. Despite showing the expected highest enrichment in adipocytes, these 468 trends additionally revealed an enrichment in smooth muscle cells, melanocytes and 469 stromal cells. Similarly, type 2 diabetes and cholesterol-related traits revealed not only 470 the expected association with hepatocytes but also with several kidney cell 471 populations ${ }^{70}$. Our analysis also pointed at the enrichment of attention deficit and 472 hyperactive disorder (ADHD) in skeletal muscle type I and type II myonuclei but not 473 in neuronal cell types, suggesting an intriguing link between this pathology and motor 474 abnormalities (Fig. 5a). In this regard for example, ocular muscle hyperactivity is an 
475 accompanying sign of ADHD and might be a major trigger for the disease rather than

476 a consequence ${ }^{71}$.

477 Besides the association of complex human traits to cell types stated above, we 478 also generated a correlation map of mutant genes causing human genetic diseases with 479 all cell types annotated in our monkey dataset (Extended Data Fig. 37 and 480 Supplementary Table 5b). As expected, genes related to retinitis pigmentosa were 481 specifically expressed in monkey photoreceptors, while genes related to porphyria were 482 found associated to erythroblasts. This shows that our dataset can predict cell types that 483 are directly affected in human genetic diseases. In addition, we compared the 484 interspecies distribution of a panel of genes related to human neurological diseases 485 using snRNA-seq data for mouse, monkey and human neocortex ${ }^{46}$. Notably, for most 486 genes, we observed a generally higher correlation of the expression in specific cell types 487 between human and monkey than between human and mouse (Fig. 5b). However, some 488 diseases also appeared to be related to different cell types in monkey compared to 489 human. For instance, distal neuropathy caused by mutations in $H S P B 8^{72}$ was enriched 490 in $\mathrm{CNRI}^{+}$inhibitory neurons in human while being enriched in astrocytes in monkey 491 and mouse. Similarly, ataxia telangiectasia caused by mutations in $A T M$ was mostly 492 enriched in oligodendrocytes ${ }^{73}$ in human while in monkey and mouse it was enriched 493 in $P V A L B^{+}$and $L A M P 5^{+}$inhibitory neurons, respectively.

494 Our analysis thus highlights the potential for modelling human diseases in 495 species phylogenetically closer to humans and underlines that differences will still exist. 496 Further scrutiny of GWAS datasets and gene mutations and wider comparisons between 497 species will provide additional relevant observations.

\section{DISCUSSION}

500 Despite the enormous potential, few NHP tissues have been profiled to date at the 501 single-cell level and the use of different species, experimental conditions and platforms makes comparisons challenging ${ }^{20,74,75}$. To address this, we have generated the first 
503 version of a large single-cell transcriptomic atlas for a NHP widely used in research

504 studies, Macaca fascicularis, and an expandable and interactive database 505 (https://db.cngb.org/nhpca/) to facilitate its exploration. The current version of our atlas 506 provides a comprehensive and integrated overview of gene expression in 106 cell types 507 extracted from 43 tissue types. Specialized tissues such as skin, thymus, testis and some 508 parts of the gastrointestinal tract, as well as increased cell numbers for some of the 509 already profiled ones, will be added in future releases. Cell type identification relied on 510 previously reported markers and gene expression profiles. Therefore, although we 511 identified most (if not all) known cell types in these tissues, our current annotations are 512 likely to benefit from deeper sub-clustering and further revision.

513 We provide a detailed description of individual tissue single-cell composition and 514 a comparison of common cell types across all sequenced tissues. This information will 515 be particularly valuable for understanding tissues that have either not been profiled at 516 all at the single-cell level in human (e.g., diaphragm, tongue and salivary gland) or lack 517 enough cell numbers (e.g., liver, gallbladder and substantia nigra), and for prediction 518 of human disease susceptibilities. Regarding the latter, we have identified an 519 unexpected link between ADHD and muscle function. ADHD is a polygenic and 520 multifactorial disorder associated with hyperactivity and motor coordination 521 abnormalities that are thought to have a neurological origin ${ }^{76}$. Our data support the 522 possibility that skeletal muscle rather than the nervous system may be a direct driver of 523 ADHD pathogenesis ${ }^{77}$. Similarly, as part of the analysis for virus receptors and co524 receptors, we provide a comprehensive map of $A C E 2^{+} / T M P R S S 2^{+}$double positive cells 525 throughout the monkey body that may be useful to understand COVID-19 pathogenesis 526 in human ${ }^{59,61}$. In particular, the link between IL6, STAT transcription factors and ACE2 527 expression could explain the reported positive effects of tocilizumab, a humanized 528 monoclonal antibody against IL6R for the treatment of patients with severe COVID$52919^{78}$. On the other hand, our study shows significant interspecies differences in cell 530 type-specific gene expression with potentially important functional consequences. For 
531 example, the distribution of ACE2 and TMPRSS2 across different cell types is not

532 identical between monkey and human and this could influence the disease course.

533 Moreover, in the context of the survey of Wnt pathway components we have identified

$534 L G R 5^{+}$renal cells with progenitor characteristics that are seemingly absent in human

535 and mouse based on analysis of reported datasets. This is relevant because the kidney

536 has limited regenerative capacity in mammals ${ }^{79}$. During embryonic development

$537 L G R 5^{+}$cells located at the junction between the ureteric bud (source of the collecting

538 tubule and connecting tubule) and the metanephric blastema are responsible for

539 nephrogenesis, but they quickly disappear after birth ${ }^{45}$. Their persistence in adult

540 monkey kidney suggests a higher regenerative capacity compared to other species,

541 which if true raises the hope of activating a similar mechanism in human ${ }^{80}$. Similarly,

$542 \mathrm{LGR5}^{+}$cells in the neocortex correspond mainly to OPC in monkey and to

543 oligodendrocytes and to a lesser extent OPC in human, whereas in mouse inhibitory

544 neurons are more highly enriched. This finding is consistent with the knowledge that

545 Wnt activity regulates OPC and oligodendrocyte function and differentiation ${ }^{81}$ but

546 suggest interspecies differences in the mode of action. Likewise, the expression of

547 LGR5 in skeletal slow-twitch myofibers, and LGR6 in the pituitary gland and heart, is

548 intriguing. During development, Wnt activity regulates skeletal myogenesis and

549 myofiber typing ${ }^{82}$, cardiomyocyte proliferation ${ }^{83}$ and pituitary gland growth ${ }^{84}$, but little

550 is known about the adult. The functional implications of these and other related findings

551 and the extent to which the patterns differ between monkey and other mammalian

552 species will require further study. Finally, interspecies comparison of single-cell gene

553 expression in neocortex highlights the problems associated with modelling neurological

554 diseases in rodents and suggests that a cautious approach should also be taken when

555 studying NHP. Additional comparisons with other human and mouse single-cell/nuclei

556 datasets will provide a more comprehensive, body-wide picture of differences in

557 disease vulnerability among the three species. 
In the future, with efforts from us and scientists worldwide, the NHPCA database

559 will be extended with additional single-cell datasets generated from disease modelling

560 studies, spontaneously developed diseases (e.g., diabetes or cardiomyopathy) and aging.

561 Adding other layers of single-cell -omics studies, in particular scATAC-seq and

562 spatially resolved transcriptomics ${ }^{85}$ for all tissues presented here, will help characterize

563 cell states and the interactions between different cell types more accurately. Proof of

564 principle is the kidney scATAC-seq dataset included here. In addition, it will be

565 important to compare our Macaca fascicularis atlas with datasets from other non-

566 endangered NHP species such as Macaca mulatta (rhesus monkey), Callithrix jacchus

567 (marmoset monkey) $)^{86}$ and Microcebus murinus (mouse lemur) ${ }^{10,13}$. Altogether, this

568 information will be instrumental for understanding primate evolution and human

569 disease.

FIGURE LEGENDS

Figure 1. Generation of a single-cell atlas across 43 tissues of Macaca fascicularis monkey.

(a) Schematic representation of monkey tissues analyzed in this study (top left panel). A total of 43 tissues were collected from three male and three female 6year-old monkeys. UMAP visualization of the global clustering indicating all single cells from the dataset colored by tissue (top middle panel) and bar plot showing the number of cells/nuclei profiled for every tissue after passing the quality control (top right panel). $\mathrm{N}=1,084,164$ individual nuclei/cells analyzed.

(b) UMAP visualization of tissues grouped by specific systems such as immune system (bone marrow, peripheral blood, spleen, tonsil and lymph node), digestive system (colon, duodenum, esophagus, gallbladder, liver, stomach and 
tongue) and female reproductive system (fallopian tube, ovary, uterus and vagina).

(c) UMAP visualization of all clusters colored by major cell types. A total of 106 cell clusters were identified in the dataset. Cell type annotation for all major clusters is provided in the right-hand side legend. SERPINE1 was used to

Figure 2. Characterization of skeletal myofibers and mesothelial cells.

(a) UMAP visualization of the global clustering of skeletal muscle cells annotated discriminate two distinct cluster of hepatocytes. in our dataset. Clusters are colored by tissue (abdominal wall, diaphragm, esophagus and tongue). Due to their low number, fallopian tube, vagina and tonsil skeletal cells were excluded from this analysis. Endothelial and immune cells were not included in this analysis.

(b) UMAP representation of all re-clustered skeletal muscle cells colored by subtype.

(c) UMAP visualization of specific markers used to identify type I (MYH7), type

(d) Stacked bar plot representing the proportion of skeletal muscle nuclei (myonulcei subtypes type I, type IIa, type IIb, MTJ and NMJ, and also satellite IIa (MYH2) and type IIb myonuclei (GPD2), FAP (LVRN), MTJ (NAV3 and COL22A1), NMJ (ETV5 and MUSK) and satellite cells (PAX7), as shown in b. Due to their small proportions, the latter three populations are highlighted by a red arrow. cells and FAP) in the indicated tissues.

(e) Heatmap showing DEG among the skeletal muscle populations highlighted in d.

(f) Bubble plot showing DEG for each of the myonuclei subtypes comparing different tissues. 
(g) UMAP visualization of mesothelial cells from the selected tissues (bladder, diaphragm, fallopian tube, lung, ovary and visceral adipose tissue). Two different clusters of mesothelial cells in visceral adipose tissue are indicated by the red dotted line.

(h) Violin plot showing the differential expression of mesothelial and immune markers in the two visceral adipose tissue clusters highlighted by the red dotted line in panel $\mathbf{g}$.

(i) UMAP visualization of three different clusters of mesothelial cells from the ovary (left panel). Mesothelial cells (Meso), surface epithelial (Surface epi) and progenitor-like epithelial (Prog-like epi) cells are highlighted in red, blue and yellow, respectively.

(j) UMAP visualization of $L G R 5$ expression in ovarian cells.

(k) Violin plot showing the DEG among the three populations of ovarian cells highlighted in the UMAP.

\section{Figure 3. Analysis of $L G R 5^{+}$cells across all monkey tissues.}

(a) UMAP visualization of $L G R 5$ expression across all tissues profiled in this study. The bubble plot on the right shows the LGR5 expression ratio in the indicated cell types.

(b) Co-embedding of kidney snRNA-seq (highlighted in blue) and scATAC-seq (highlighted in red) datasets.

(c) UMAP visualization of integrated kidney snRNA- and scATAC-seq data. Cell clusters are colored according to cell identity. Abbreviations: DCTC, distal convoluted tubule cells; Endo, endothelial cells; LOH, loop of Henle; mDC, myeloid-derived dendritic cells; Myofibro, myofibroblasts.

(d) UMAP visualization of LGR5 across kidney cell types and ArchR track visualization of aggregate scATAC-seq signals on the LGR5 locus in each cell 

$L G R 5^{+}$cells in each cell type of kidney. (scale bar $20 \mu \mathrm{m}$ ). The bottom panel represents a magnification of the area indicated by the white box in the top panel.

(f) GO analysis showing the pathways associated to the DEGs obtained by comparing $L G R 5^{+}$cells from DCTC, ascending and descending LOH. The UMAP and the barplot on the right highlight the presence and the percentage of $L G R 5^{+}$cells co-expressing the progenitor markers PAX2, TNFRSF19 and LHX2.

(h) UMAP visualization of $L G R 5, M Y H 2$ and $M Y H 7$ across all skeletal muscle cell types. The blue dotted line in the left panel indicates all clusters belonging to the diaphragm while the one in the right panel indicates $L G R 5^{+}$cells.

(i) Representative image of smFISH detection for $L G R 5, M Y H 7$ and their coexpression in skeletal myonuclei of the diaphragm (scale bar $20 \mu \mathrm{m}$ ). The panel of the right is a magnification of the area indicated by the white box.

\section{Figure 4. Global analysis of $A C E 2$ and TMPRSS2 across monkey tissues.}

(a) Heatmap showing the expression of entry receptors for a selection of the most common viruses (indicated on the left) in all cell clusters annotated in our dataset (indicated at the bottom).

(b) UMAP visualization of $A C E 2$ (top) and TMPRSS2 (bottom) expression in all single cells from our dataset. The bubble plot next to each UMAP shows the expression levels of ACE2 and TMPRSS2 in the indicated cell types. The color 

proportion of expressing cells.

(c) UMAP projection of $A C E 2^{+} / T M P R S S 2^{+}$cells (highlighted in yellow). The bar plot on the right represents the ratio of cells that co-express both genes.

(d) UMAP visualization of $A C E 2$ in the integrated scATAC-seq and snRNA-seq from monkey kidney.

(e) ArchR track visualization of aggregate scATAC-seq signals on the ACE2 locus in each on the annotated cell types of the kidney. Predicted binding of human

Figure 5. Association of monkey transcriptomic profiles with human common

(a) Heatmap showing the association of selected common human traits and diseases (indicated on the right) with the cell types (indicated at the bottom) annotated in our dataset. The colored boxes indicate enriched specific patterns related to human traits/diseases subtypes.

(b) Heatmap showing the enrichment of genetic diseases related to the central nervous system in human, monkey and mouse neocortex snRNA-seq datasets.

Extended Data Figure 1. Quality control analysis of gender and individual effect. UMAP visualization of single-cell profiles for selected tissues to calculate the 
diaphragm (F1 and M2), gallbladder (F1 and F3), kidney (F1 and F2), lung (F1 and F3), salivary gland (F1 and F3), subcutaneous (F1 and M2) and visceral adipose (F1 and M2) tissues, and three for liver (F1, F2 and F3).

\section{Extended Data Figure 2. Global clustering of different systems.}

UMAP visualization of cell clusters in selected tissues grouped by system: cardiovascular (aorta, carotid and heart), endocrine (adrenal, pancreas, pineal, pituitary and thyroid glands), skeletal (abdominal wall and diaphragm), central nervous (cerebellum, neocortex, pigmentary epithelium choroid plexus, retina and spinal cord), respiratory (bronchus, lung and trachea) and urinary (bladder and kidney). Adipose tissues (subcutaneous and visceral) are also shown grouped. Clusters shown in every plot are colored by tissue. Abbreviation: pigmentary epi, pigmentary epithelium and choroid plexus.

\section{Extended Data Figure 3. Global profiling of individual monkey tissues - 1.}

UMAP projection of the global clustering indicating the distribution of all single cells (highlighted in yellow) from individual tissues for abdominal wall, adrenal gland, aorta, bladder, bone marrow, bronchus, carotid, cerebellum, colon, diaphragm, duodenum and epididymis.

\section{Extended Data Figure 4. Global profiling of individual monkey tissues - 2.} UMAP projection of the global clustering indicating the distribution of all single cells (highlighted in yellow) from individual tissues for esophagus, fallopian tube, gallbladder, heart, kidney, liver, lung, lymph node, neocortex, ovary, pancreas and PBMC.

\section{Extended Data Figure 5. Global profiling of individual monkey tissues - 3 .}


UMAP projection of the global clustering indicating the distribution of all single cells (highlighted in yellow) from individual tissues for pigmentary epithelium choroid plexus, pineal gland, pituitary gland, prostate, retina, salivary gland, spermaduct, spinal cord, spleen, stomach, subcutaneous adipose tissue and substantia nigra.

Extended Data Figure 6. Global profiling of individual monkey tissues - 4 . cells (highlighted in yellow) from individual tissues for thyroid, tongue, tonsil,

\section{Extended Data Figure 7. Cluster annotations - 1.}

UMAP visualization of cell clusters in the abdominal wall, adrenal gland, aorta, bladder, bone marrow, bronchus, carotid, cerebellum, colon, diaphragm, duodenum and epididymis. The name of the population in each cluster and the total number of cells profiled for every tissue are indicated in every plot. Abbreviations: Adipo, adipocytes; Astro, astrocytes; AT1, alveolar type 1 cells; AT2, alveolar type 2 cells; BC, B cells; CLP, common lymphoid progenitors; CMP, common myeloid progenitors; Endo, endothelial cells; Epi, epithelial cells; Ery, erythroblasts; FAP, fibroadipogenic progenitors; GMP, granulocyte monocyte progenitors; Macro, macrophages; mDC, myeloid derived dendritic cells; MEP, megakaryocyte erythrocyte progenitors; Meso, mesothelial cells; Mol interneu, molecular interneurons; Mono, monocytes; MTJ, myotendinous junction; Myofibro, myofibroblasts; NK, natural killers; NKT, natural killer T cells; NMJ, neuromuscular junction; Oligo, oligodendrocytes; OPC, oligodendrocyte progenitor cells; SMC, smooth muscle cells; TC, T cells. 
UMAP visualization of cell clusters in the esophagus, fallopian tube, gallbladder, heart, kidney, liver, lung, lymph node, neocortex, ovary, pancreas and PBMC. The name of the population in each cluster and the total number of cells profiled for every tissue are indicated in every plot. Abbreviations: Adipo, adipocytes; Astro, astrocytes; AT1, alveolar type 1 cells; AT2, alveolar type 2 cells; BC, B cells; Endo, endothelial cells; Epi, epithelial cells; EX, excitatory neurons; Hep, hepatocytes; IN, inhibitory neurons; IDC, lymphoid derived dendritic cells; LOH, loop of Henle cells; Lymph prog, lymphoid progenitors; Macro, macrophages; mDC, myeloid derived dendritic cells; Meg, megakaryocytes; Meso, mesothelial cells; Mono, monocytes; Myofibro, myofibroblasts; NK, natural killers; NKT, natural killer T cells; NMJ, neuromuscular junction; Oligo, oligodendrocytes; OPC, oligodendrocyte progenitor cells; Prog-like epi, progenitor-like epithelial cells; SMC, smooth muscle cells; TC, T cells.

\section{Extended Data Figure 9. Cluster annotations - 3.}

UMAP visualization of cell clusters in the pigmentary epithelium choroid plexus, pineal gland, pituitary gland, prostate, retina, salivary gland, spermaduct, spinal cord, spleen, stomach, subcutaneous adipose tissue and substantia nigra. The name of the population in each cluster and the total number of cells profiled for every tissue are indicated in every plot. Abbreviations: Adipo, adipocytes; Astro, astrocytes; BC, B cells; DAN, dopaminergic neurons; DC, conventional dendritic cells; Endo, endothelial cells; Epi, epithelial cells; EX, excitatory neurons; IN, inhibitory neurons; Macro, macrophages; Mono, monocytes; Myofibro, myofibroblasts; Neutro, neutrophils; NK, natural killers; NKT, natural killer T cells; Oligo, oligodendrocytes; OPC, oligodendrocyte 
UMAP visualization of cell clusters in the thyroid, tongue, tonsil, trachea, uterus, vagina and visceral adipose tissue. The name of the population in each cluster and the total number of cells profiled for every tissue are indicated in every plot. Abbreviations: Adipo, adipocytes; BC, B cells; Endo, endothelial cells; IDC, lymphoid derived dendritic cells; LOH, loop of Henle; Macro, macrophages; mDC, myeloid derived dendritic cells; Meso, mesothelial cells; Mono, monocytes; NK, natural killers; NMJ, neuromuscular junction; SMC, smooth muscle cells; TC, T cells.

Extended Data Figure 11. Selected markers for cell cluster annotations. all cell clusters identified in every tissue of this dataset.

Extended Data Figure 12. UMI and gene numbers of the sequenced tissues and annotated cell types.

(a) Boxplot indicating the number of UMI (top) and genes (bottom) in each tissue of the dataset.

(b) Boxplot indicating the number of UMI (top) and genes (bottom) detected in each of the major annotated cell types shown in Figure 1c.

Extended Data Figure 13. Cell numbers and proportions among the sequenced tissues. ratio of each cell type detected in every tissue.

\section{Extended Data Figure 14. Unique and shared cell populations.}


(a) UMAP projection of the global clustering showing the expression of specific markers for cerebellum granule cells $(G A B R A 6)$, hepatocytes $(A L B)$, salivary gland acinar cells (PRR27), epididymis stereociliated cells (ROS1), pancreatic alpha cells $(G C G)$ and fasciculata cells of the adrenal gland (CYP11A1).

(b) UMAP projection of the global clustering showing the expression of panmarkers of endothelial (FLT1), stromal $(D C N)$, immune (PTPRC), skeletal myonuclei $(T T N)$, adipocytes (ADIPOQ) and mesothelial cells (ITLN1) that are shared across tissues.

\section{Extended Data Figure 15. Global analysis of common cell types.}

UMAP visualization of (a) stromal cells $(\mathrm{n}=35,415)$, (b) macrophages $(\mathrm{n}=$ 10,929), (c) endothelial cells $(\mathrm{n}=37,640)$ and (d) smooth muscle cells $(\mathrm{n}=$ $24,175)$ from all analyzed monkey tissues. Tissues with low numbers of the selected cell types were excluded. Cell clusters are colored by tissue. The heatmap on the right shows tissue-specific DEG for (e) stromal cells, (f) macrophages, (g) endothelial cells and (h) smooth muscle cells.

\section{Extended Data Figure 16. Analysis of skeletal myonuclei molecular signatures.}

(a) Bubble plot indicating tissue-specific enriched GO terms in type I myonuclei from abdominal wall, diaphragm and tongue.

(b) Bubble plot indicating tissue-specific enriched GO terms in type IIa myonuclei from diaphragm, esophagus and tongue.

(c) Bubble plot indicating tissue-specific enriched GO terms in type IIb myonuclei from abdominal wall and diaphragm.

\section{Extended Data Figure 17. Global analysis of adipocyte populations.}

(a) UMAP visualization of mature adipocyte and adipocyte progenitors from visceral (VAT) and subcutaneous (SAT) adipose tissues. Data were grouped 
together and re-clustered either by tissue type (on the left) or by cell type (on the right).

(b) UMAP visualization of specific markers for mature adipocytes $(A D I P O Q)$ or adipocyte progenitors (CD34).

(c) UMAP visualization of markers for tissue-specific (ITLN1 and FOS), cell-type specific (SLC11A1, SPOCK3,WT1, ESR1, CXCL14, APOD, CFD, DPP4 and NOX4) or cycling markers (MKI67).

(d) Heatmap indicating the DEG in all clusters identified in $\mathbf{a}$.

Extended Data Figure 18. Global analysis of LGR4, LGR6 and LGR5/LGR6 coexpression across monkey tissues.

(a) UMAP visualization of LGR6 across all tissues profiled in this study. The bubble plot on the right shows the LGR6 expression ratio in the indicated cell types.

(b) UMAP visualization of LGR5 and LGR6 co-expression across all tissues profiled in this study. The barplot on the right shows the co-expression ratio in the indicated cell types.

(c) UMAP visualization of $L G R 4$ across all tissues profiled in this study. The bubble plot on the right shows the LGR4 expression ratio in the indicated cell types.

Extended Data Figure 19. Global analysis of $L G R 5$ and $L G R 6$ across monkey tissues - 1 . Bubble plot (left) showing the ratio of $L G R 5^{+}, L G R 6^{+}$and $M K I 67^{+}$cells in the annotated cell types for each tissue and UMAP visualization (right) of $L G R 5$, LGR6 and MKI67 in abdominal wall, adrenal gland, aorta, bladder, bone marrow, bronchus, carotid, cerebellum, colon, diaphragm and duodenum. 
861 Extended Data Figure 20. Global analysis of LGR5 and LGR6 across monkey

862 tissues -2 .

863 Bubble plot (left) showing the ratio of $L G R 5^{+}, L G R 6^{+}$and $M K I 67^{+}$cells in the 864 annotated cell types for each tissue and UMAP visualization (right) of $L G R 5$, 865 LGR6 and MKI67 in epididymis, esophagus, fallopian tube, gallbladder, heart, 866 kidney, liver, lung, lymph node and ovary.

867

Extended Data Figure 21. Global analysis of $L G R 5$ and $L G R 6$ across monkey tissues -3 . Bubble plot (left) showing the ratio of $L G R 5^{+}, L G R 6^{+}$and $M K I 67^{+}$cells in the annotated cell types for each tissue and UMAP visualization (right) of $L G R 5$, LGR6 and MKI67 in pancreas, PBMCs, pigmentary epithelium choroid plexus (indicated as pigmentary epi), pineal gland, pituitary gland, prostate, retina, salivary gland, spermaduct, spinal cord and spleen.

Extended Data Figure 22. Global analysis of LGR5 and LGR6 across monkey Bubble plot (left) showing the ratio of $L G R 5^{+}, L G R 6^{+}$and $M K I 67^{+}$cells in the annotated cell types for each tissue and UMAP visualization (right) of $L G R 5$, LGR6 and MKI67 in stomach, subcutaneous adipose tissue, substantia nigra,

Extended Data Figure 23. Kidney snRNA-seq and scATAC-seq dataset integration. thyroid, tongue, tonsil, trachea, uterus, vagina and visceral adipose tissue.

(a) Violin plot showing the expression of selected markers used to annotate the kidney cell clusters from snRNA-seq data.

(b) ArchR track visualization of aggregate scATAC-seq signals on the locus of the selected marker genes indicated in a. Abbreviations: DCTC, distal convoluted 
888

889

890

891

892

893

894

895

896

897

898

899

900

901

902

903

904

905

906

907

908

909

910

911

912

913

tubule cells; Endo, endothelial cells; LOH, loop of Henle; Myofibro, myofibroblasts.

\section{Extended Data Figure 24. LGR5 analysis in integrated human, monkey and mouse}

\section{kidney data.}

(a) UMAP visualization of cell clusters in human (left), monkey (middle) and mouse (right) kidney snRNA-seq datasets. The annotation of each cluster in provided in the legend at the bottom. Abbreviations: Endo, endothelial cells; LOH, loop of Henle; mDC, myeloid dendritic cells; Myofibro, myofibroblasts.

(b) UMAP visualization of LGR5 in human (left), monkey (middle) and mouse (right) kidney.

(c) Bubble plot showing the ratio and expression levels of $L G R 5$ and DCTC marker SLC12A3 in human, monkey and mouse kidney datasets. The color of each bubble represents the level of expression and the size indicates the proportion of expressing cells.

\section{Extended Data Figure 25. LGR5 analysis in integrated human, monkey and mouse} neocortex data.

(a) UMAP visualization of cell clusters in human (left), monkey (middle) and mouse (right) neocortex snRNA-seq datasets. The annotation of each cluster is provided in the legend at the bottom. Abbreviations: Astro, astrocytes; Endo, endothelial cells; IN, inhibitory neurons; OPC, oligodendrocyte progenitor cells; EX, excitatory neurons; Oligo, oligodendrocytes.

(b) UMAP visualization of LGR5 in human (left), monkey (middle) and mouse (right) neocortex. OPC and oligodendrocytes are indicated by a red and yellow dotted circle, respectively. 
(c) Bubble plot showing the ratio and expression levels of $L G R 5$ and PDGFRA in human, monkey and mouse neocortex. The color of each bubble represents the level of expression and the size indicates the proportion of expressing cells.

(d) Monocle 2 pseudotime-ordered trajectory of OPC (labelled in orange) maturation towards mature oligodendrocytes (labelled in blue).

(e) Monocle 2 pseudotime analysis showing the expression of OPC markers (LGR5, $O L I G 2$ and $P D G F R A$ ) and the oligodendrocytes marker PLP1.

(f) Representative image of immunofluorescence staining for PDGFRA (red) and LGR5 (green), respectively, and their co-expression in OPC of monkey neocortex (scale bar $20 \mu \mathrm{m}$ ). The smaller panel at the bottom is a magnification

Extended Data Figure 26. Analysis of LGR6 expression in monkey heart and

(a) UMAP visualization of LGR6 across all muscle cell types annotated in our dataset, as displayed in Figure 3g. The dotted red line indicates a cluster of muscle cells belonging to the heart. The red arrows indicate $L G R \sigma^{+}$cells in aorta, carotid, ovary and vagina.

(b) Representative image of smFISH detection for LGR6 in heart myonuclei (scale

(c) UMAP visualization of cell clusters in human (left), monkey (middle) and bar $40 \mu \mathrm{m}$ ). The bottom right panel is a magnification of the area indicated by the white box.

$$
\text { mouse (right) heart snRNA-seq datasets. The annotation of each cluster is }
$$
provided in the legend at the bottom. Abbreviations: Endo, endothelial cells; Macro, macrophages; SMC, smooth muscle cells.

(d) UMAP visualization of LGR6 in human (left), monkey (middle) and mouse (right) heart. 
(e) UMAP visualization of LGR6 expression in pituitary gland highlighting the highest expression in folliculostellate cells.

(f) UMAP visualization of $S O X 2, P A X 6, C D 44$ and $C X C R 4$ in folliculostellate cells as indicated by the black box.

(g) Barplot showing GO terms associated to the DEGs in folliculostellate cells of pituitary gland.

Extended Data Figure 27. Analysis of $W N T 9 B$ and Wnt pathway gene module in

(a) Heatmap showing the expression of all receptors and ligands of the Wnt pathway in the annotated cell populations of the kidney.

(b) Network plots showing cell-cell communications based on ligand-receptor interactions calculated by CellphoneDB.

(c) UMAP visualization of $W N T 9 B$ expression in monkey kidney.

(d) ArchR track visualization of aggregate scATAC-seq signals on the $W N T 9 B$ locus in each on the annotated cell types. The bar plot at the bottom indicates the ratio $(\%)$ of $W N T 9 B^{+}$cells in each cell type of kidney.

(e) Bubble plot showing the ratio and expression levels of $W N T 9 B$ and principal

Extended Data Figure 28. Global analysis of the Wnt pathway gene module in monkey diaphragm and epididymis. pathway in the annotated cell populations of the diaphragm. 
(b) Network plots showing cell-cell communication based on ligand-receptor interactions calculated by CellphoneDB in the diaphragm dataset. Abbreviations: Adipo, adipocytes; Endo, endothelial cells; Macro, macrophages; Meso, mesothelial cells; NMJ, neuromuscular junctions.

(c) Heatmap showing the expression of all receptors and ligands of the Wnt pathway in the annotated cell populations of the epididymis.

(d) Network plots showing cell-cell communication based on ligand-receptor interactions calculated by CellphoneDB in the epididymis dataset. Abbreviations: Adipo, adipocytes; Endo, endothelial cells; SMC, smooth muscle cells; TC, T cells.

Extended Data Figure 29. Global analysis of the Wnt pathway gene module in monkey fallopian tube and liver.

(a) Heatmap showing the expression of all receptors and ligands of the Wnt pathway in the annotated cell populations of the fallopian tube.

(b) Network plots showing cell-cell communication based on ligand-receptor interactions calculated by CellphoneDB in the fallopian tube dataset. Abbreviations: Endo, endothelial cells; epi, epithelial cells; Meso, mesothelial cells; Mono, monocytes; SMC, smooth muscle cells; TC, T cells.

(c) Heatmap showing the expression of all receptors and ligands of the Wnt pathway in the annotated cell populations of the liver.

(d) Network plots showing cell-cell communication based on ligand-receptor interactions calculated by CellphoneDB in the liver dataset. Abbreviations: BC, B cells; Endo, endothelial cells; hep, hepatocytes; mDC, myeloid derived dendritic cells; Mono, monocytes; TC, T cells.

Extended Data Figure 30. Global analysis of the Wnt pathway gene module in monkey neocortex and ovary. 
(a) Heatmap showing the expression of all receptors and ligands of the Wnt pathway in the annotated cell populations of the neocortex.

(b) Network plots showing cell-cell communication based on ligand-receptor interactions calculated by CellphoneDB in the neocortex dataset. Abbreviations: Astro, astrocytes; Endo, endothelial cells; EX, excitatory neurons; IN, inhibitory neurons; Oligo, oligodendrocytes; OPC, oligodendrocyte progenitor cells.

(c) Heatmap showing the expression of all receptors and ligands of the Wnt pathway in the annotated cell populations of the ovary.

(d) Network plots showing cell-cell communication based on ligand-receptor interactions calculated by CellphoneDB in the ovary dataset. Abbreviations:

Extended Data Figure 31. Global analysis of the Wnt pathway gene module in other monkey heart and pituitary gland.

(a) Heatmap showing the expression of all receptors and ligands of the Wnt pathway in the annotated cell populations of the heart.

(b) Network plots showing cell-cell communication based on ligand-receptor interactions calculated by CellphoneDB in the pituitary gland dataset. Abbreviations: Endo, endothelial cells; Myofibro, myofibroblasts.

(c) Heatmap showing the expression of all receptors and ligands of the Wnt pathway in the annotated cell populations of the pituitary gland.

(d) Network plots showing cell-cell communication based on ligand-receptor 
1025 Extended Data Figure 32. Global analysis of virus entry receptors across monkey

1026 tissues.

1027 Heatmap showing the expression of entry receptor for most common viruses

1028 (shown on the right) in the indicated cell types (shown at the bottom).

1029

Extended Data Figure 33. Analysis of $A C E 2$ and TMPRSS2 expression across

monkey tissues -1 .

UMAP visualization of $A C E 2$ (left), TMPRSS2 (middle) and $A C E 2^{+} / T M P R S S 2^{+}$

1033

1034

(right) in abdominal wall, adrenal gland, aorta, bladder, bone marrow, bronchus,

1035 carotid, cerebellum, colon, diaphragm, duodenum, epididymis, esophagus,

1036

Extended Data Figure 34. Analysis of $A C E 2$ and TMPRSS2 expression across

monkey tissues -2 .

UMAP visualization of $A C E 2$ (left), TMPRSS2 (middle) and $A C E 2^{+} / T M P R S S 2^{+}$ pigmentary epi), pineal gland, pituitary gland, prostate, retina, salivary gland, spermaduct, spinal cord, spleen, stomach, subcutaneous adipose tissue, substantia nigra, thyroid, tongue, tonsil, trachea, uterus, vagina and visceral

Extended Data Figure 35. Comparative analysis of $A C E 2$ and TMPRSS2 expression in monkey and human.

(a) Bubble plot showing the ratio and expression levels of ACE2 and TMPRSS2 in gallbladder, kidney, liver and lung in monkey and human. The color of each bubble represents the level of expression and the size indicates the proportion of expressing cells. 
(b) UMAP visualization of $I L 6 R, I L 1 R I$ and $I L 1 R A P$ expressing in monkey kidney (top). The UMAP in the bottom represent the co-expression of $A C E 2$ and IL6R, ILIRI and ILIRAP in monkey kidney. Double positive cells are indicated in yellow.

(c) Schematic diagram of the potential mechanism for SARS-CoV-2 spreading through gallbladder, kidney, liver and lung. Kidney proximal tubule cells within the nephron are among the highest ACE2 expressing cells. After virus contact, IL6R stimulates an immune response that, through the activation of STAT transcription factors, potentiates a paracrine positive feedback loop that

Extended Data Figure 36. Expression of genes associated with human common Heatmap showing the association of common human traits and diseases from the UK Biobank (indicated on the right) with the cell types (indicated at the bottom) annotated in our dataset.

Extended Data Figure 37. Association of monkey cell type-specific transcriptomic profiles with human genetic diseases.

1077 Supplementary Table 1. Description of all tissues profiled, cell types and markers 
1080 Supplementary Table 2. Global analysis of common cell types and tissue-specific

$1081 \quad$ signatures

1082

1083 Supplementary Table 3. Global distribution of LGR5, LGR6 and MKI67

1084 expression

1085

1086 Supplementary Table 4. Analysis of the expression of common virus and SARS-

1087 Cov-2 receptors

1088

1089

Supplementary Table 5. Correlation of GWAS traits and human genetic diseases

1090

with monkey cell types

1091

1092

1093

METHODS

1094

\section{Ethics statement}

1096 This study was approved by the Institutional Review Board on Ethics Committee of

1097 BGI (permit no. BGI-IRB19125).

1098

\section{Collection of monkey tissues}

1100 A total of three females and three males, approximately 6-year-old, cynomolgus

1101 monkeys were obtained from Huazhen Laboratory Animal Breeding Centre and Hubei

1102 Topgene Biotechnology (Guangzhou, China). Animals were anesthetized with

1103 ketamine hydrochloride $(10 \mathrm{mg} / \mathrm{kg})$ and sodium pantabarbital $(40 \mathrm{mg} / \mathrm{kg})$ injection

1104 before being euthanized by exsanguination. Tissues were isolated and placed on the

1105 ice-cold board for dissection. A total of 43 whole tissues were isolated: abdominal wall,

1106 adrenal gland, aorta and carotid arteries, bladder, bone marrow, bronchia, cerebellum,

1107 colon, diaphragm, duodenum, epididymis, esophagus, fallopian tube, gallbladder, heart, 
1108 kidney, liver, lung, lymph node, neocortex, ovary, pancreas, PBMC, pigmentary

1109 epithelium choroid plexus, pineal gland, pituitary gland, prostate, retina, salivary gland,

1110 spermaduct, spinal cord, spleen, stomach, subcutaneous adipose tissue, substantia nigra,

1111 thyroid gland, tongue, tonsil, trachea, uterus, vagina and visceral adipose tissue. Each

1112 tissue (except for bone marrow, peripheral blood and tissues on which enzymatic

1113 digestion was performed) was cut into 5-10 pieces of roughly 50-200 mg each. Samples

1114 were transferred to cryogenic vials (Corning, \#430488), then quickly frozen in liquid

1115 nitrogen and finally stored until nuclear extraction was performed. PBMC and bone

1116 marrow cells were isolated from heparinized venous blood using a Lymphoprep ${ }^{\mathrm{TM}}$

1117 medium (STEMCELL Technologies, \#07851) according to standard density gradient

1118 centrifugation methods. Cells from those two tissues were resuspended in $90 \%$ FBS,

1119 10\% DMSO (Sigma Aldrich, \#D2650) freezing media and frozen using a Nalgene ${ }^{\circledR}$

1120 Mr. Frosty ${ }^{\circledR}$ Cryo $1^{\circ} \mathrm{C}$ Freezing Container (Thermo Fisher Scientific, \#5100-0001) in

$1121 \mathrm{a}-80^{\circ} \mathrm{C}$ freezer for 24 hours before being transferred to liquid nitrogen for long-term

1122 storage.

1123

\section{Single-nucleus/cell suspension preparation}

1125 Single nucleus isolation was performed as described previously ${ }^{87}$. Briefly, tissues were

1126 thawed, minced and transferred to a $1 \mathrm{ml}$ Dounce homogenizer (TIANDZ) with $1 \mathrm{ml}$

1127 of homogenization buffer A containing $250 \mathrm{mM}$ sucrose (Ambion), $10 \mathrm{mg} / \mathrm{ml}$ BSA

1128 (Ambion), $5 \mathrm{mM} \mathrm{MgCl} 2$ (Ambion), $0.12 \mathrm{U} / \mu \mathrm{l}$ RNasin Plus (Promega, \#N2115), 0.12

$1129 \mathrm{U} / \mu 1$ RNasein (Promega, \#N2115) and 1x Protease Inhibitor (Roche, \#11697498001).

1130 Tissues were kept in an ice box and homogenized by 25-50 strokes of the loose pestle

1131 (Pestle A) after which the mixture was filtered using a $100 \mu \mathrm{m}$ cell strainer in to a 1.5

$1132 \mathrm{ml}$ tube (Eppendorf). The mixture was then transferred to a clean $1 \mathrm{ml}$ dounce

1133 homogenizer to which 750 ul of buffer A containing 1\% Igepal (Sigma, \#CA630) was

1134 added and the tissue was further homogenized by 25 strokes of the tight pestle (Pestle

1135 B). After this, the mixture was filtered through a $40 \mu \mathrm{m}$ strainer in a $1.5 \mathrm{ml}$ tube and 
1136 centrifuged at $500 \mathrm{~g}$ for five minutes at $4^{\circ} \mathrm{C}$ to pellet nuclei. At this stage, the pellet was

1137 resuspended in $1 \mathrm{ml}$ of buffer B containing $320 \mathrm{mM}$ Sucrose, $10 \mathrm{mg} / \mathrm{ml} \mathrm{BSA}, 3 \mathrm{mM}$

$1138 \mathrm{CaCl}_{2}, 2 \mathrm{mM} \mathrm{MgAc} 2,0.1 \mathrm{mM}$ EDTA, $10 \mathrm{mM}$ Tris-HCl, $1 \mathrm{mM}$ DTT, 1x Protease

1139 Inhibitor and $0.12 \mathrm{U} / \mu \mathrm{l}$ RNasein. This was followed by a centrifugation at $500 \mathrm{~g}$ for

1140 five minutes at $4^{\circ} \mathrm{C}$ to pellet nuclei. Nuclei were then resuspended with cell

1141 resuspension buffer at a concentration of 1,000 nuclei/ $\mu$ l for single-nucleus library

1142 preparation. Cells from lymph node, spleen, duodenum, stomach and colon were

1143 obtained from fresh tissues by enzymatic digestion. Briefly, tissues were rinsed in PBS,

1144 minced into small pieces by mechanical dissociation and incubated for 1 hour in $10 \mathrm{ml}$

1145 of DS-LT buffer $\left(0.2 \mathrm{mg} / \mathrm{ml} \mathrm{CaCl}_{2}, 5 \mu \mathrm{M} \mathrm{MgCl}_{2}, 0.2 \% \mathrm{BSA}\right.$ and $0.2 \mathrm{mg} / \mathrm{ml}$ Liberase

1146 in HBSS) at $37^{\circ} \mathrm{C}$. After this, the tissue digestion was stopped by addition of $3 \mathrm{ml}$ of

1147 FBS, followed by filtration through a $100 \mu \mathrm{m}$ cell strainer and centrifugation for 5

1148 minutes at $500 \mathrm{~g}$ at $4^{\circ} \mathrm{C}$. Samples were then filtered through a $40 \mu \mathrm{m}$ cell strainer and

1149 centrifuged for five minutes at $500 \mathrm{~g}$ at $4^{\circ} \mathrm{C}$. Pellets were then resuspended in cell

1150 resuspension buffer at 1,000 cells/ $\mu 1$ for single-cell library preparation.

\section{Single-cell/single-nucleus RNA-seq (sc/snRNA-seq)}

1153 DNBelab C Series Single-Cell Library Prep Set was utilized as previously described ${ }^{14}$.

1154 In brief, single-nucleus/cell suspensions were used for droplet generation, emulsion 1155 breakage, beads collection, reverse transcription and cDNA amplification to generate 1156 barcoded libraries. Indexed sc/snRNA-seq libraries were constructed according to the 1157 manufacturer's protocol. The concentration of sc/snRNA-seq sequencing libraries was 1158 quantified by Qubit ${ }^{\mathrm{TM}}$ ssDNA Assay Kit (Thermo Fisher Scientific, \#Q10212). The 1159 resulting libraries were sequenced using a DIPSEQ T1 or DIPSEQ T7 sequencers at 1160 the China National GeneBank (Shenzhen, China). 
1163 ScATAC-seq libraries were prepared using DNBelab C Series Single-Cell ATAC

1164 Library Prep Set ${ }^{14}$. DNA nanoballs were loaded into the patterned Nano arrays and 1165 sequenced on a BGISEQ-500 sequencer using the following read length: $50 \mathrm{bp}$ for read

11661,76 bp for read 2, inclusive of 50 bp insert DNA, 10 bp cell barcode 1, 6 bp constant 1167 sequence and $10 \mathrm{bp}$ cell barcode 2.

1169 Immunofluorescence

1170 Staining of monkey neocortex sample was conducted following standard protocol ${ }^{88}$. In

1171 brief, paraffin embedded sections were deparaffinized, incubated with primary 1172 antibodies for PDGFRa (Cell Signaling \#3174S) and LGR5 (Abcam \#ab273092) 1173 overnight at $4{ }^{\circ} \mathrm{C}$, followed by an incubation with a secondary antibody (Alexa Fluor 1174488 and Cy3, Jackson ImmunoResearch) for 30 minutes at room temperature. Slides 1175 were mounted with Slowfade Mountant+DAPI (Life Technologies, \#S36964) and 1176 sealed.

\section{Single-molecule fluorescence in situ hybridization (smFISH)}

1179 SmFISH in monkey kidney, diaphragm and heart tissues was performed using

1180 RNAScope Fluorescent Multiplex and RNAScope Multiplex Fluorescent v2

1181 (Advanced Cell Diagnostics) according to manufacturer's instructions. The following 1182 alterations were added: the thickness of paraffin section was adjusted to $5 \mu \mathrm{m}$ and target 1183 retrieval boiling time was adjusted to 15 minutes while the incubation time of Protease 1184 plus at $40^{\circ} \mathrm{C}$ was adjusted to 30 minutes. RNA smFISH probes used: LGR5 (C1), LGR6 1185 (C2), MYH7 (C2).

\section{Sc/snRNA-seq data processing}

1188 Raw sequencing reads from DIPSEQ-T1 or DIPSEQ-T7 were filtered and 1189 demultiplexed using PISA (version 0.2) (https://github.com/shiquan/PISA). Reads 1190 were aligned to Macaca_fascicularis_5.0 genome using STAR (version 2.7.4a) ${ }^{89}$ and 
1191 sorted by sambamba (version 0.7 .0$)^{90}$. For tissues sequenced with scRNA-seq, reads

1192 were aligned to the exon of mRNA as normal. For tissues sequenced with snRNA-seq,

1193 a custom 'pre-mRNA' reference was created for alignment of count reads to introns as

1194 well as to exons because of large amount of unspliced pre-mRNA and mature mRNA

1195 in the cell nucleus. Thus, each gene's transcripts in snRNA-seq was counted out by

1196 including exon and intron reads together ${ }^{91}$. In the end, cell/nucleus versus gene UMI 1197 count matrix was generated with PISA.

\section{Doublet removal}

1200 For each library, we performed doublet removal using DoubletFinder ${ }^{92}$. DoubletFinder

1201 first averages the transcriptional profile of randomly chosen cell pairs to create pseudo

1202 doublets and then predicts doublets according to each real cell's similarity in gene

1203 expression to the pseudo doublets. The doublet removal was performed according to

1204 the default parameter of DoubletFinder and the top 5\% of cells most similar to the

1205 "pseudo doublets" were excluded.

\section{Cell clustering and identification of cell types}

1208 Clustering analysis of the complete cynomolgus monkey tissue dataset was performed 1209 using Scanpy (version 1.6.0) ${ }^{93}$ in a Python environment (version 3.6). Parameters used 1210 in each function were manually curated to portray the optimal clustering of cells. In the 1211 preprocessing, cells or nuclei were filtered based on the criteria of expressing a 1212 minimum of 500 genes and genes expressed by at least three cells or nuclei were kept 1213 for the following analysis. In addition, cells or nuclei with more than $10 \%$ 1214 mitochondrial gene counts were removed. Filtered data were ln (counts per million $1215(\mathrm{CPM}) / 100+1)$ transformed. 3,000 highly variable genes were selected according to 1216 their average expression and dispersion. The number of UMI and the percentage of 1217 mitochondrial genes were regressed out and each gene was scaled by default options. 1218 Dimension reduction starts with principal component analysis and the number of 
1219 principal components used for UMAP depended on the importance of embeddings.

1220 Louvain method is then used to detect subgroups of cells. Distinguishing differential 1221 genes among clusters were ranked (Benjamini-Hochberg, Wilcoxon rank-sum test).

1222 Cell types were manually and iteratively assigned based on overlap of literature, curated 1223 and statistically ranked genes. Each tissue dataset was portrayed using the Seurat 1224 package (version 3.2.2) ) $^{94}$ in R environment (version 3.6). Data from different replicates 1225 were integrated following the standard integrated pipeline by default parameters for 1226 filtering, data normalization, dimensionality reduction, clustering and gene differential 1227 expression analysis. Finally, we annotated each cell type by extensive literature reading 1228 and searching for the specific gene expression patterns.

Differentially expressed gene (DEG) and gene ontology (GO) term enrichment analysis

1232 In the global clustering, we performed DEG analysis using the sc.pl.rank_genes_groups 1233 function in Scanpy (V1.6.0). In other studies, we used the FindMarker or 1234 FindAllMarker function in the Seurat R package (V3.2.2). Analysis of DEG comparing specific populations was performed by calculating the fold-change of the mean expression level of genes between the selected populations. DEG were defined as those

1237 with a fold-change $>2$ and adjusted $P<0.01$. GO enrichment analysis was performed 1238 using the CompareCluster function fun $=$ "enrichGO", pvalueCutoff $=0.1$, 1239 pAdjustMethod = "BH", OrgDb = org.Hs.eg.db,ontBP") of ChIPseeker R package $1240 \quad$ (v.1.22.1 $)^{95}$. Only GO terms with adjusted $P<0.05$ were retained.

\section{Analysis of inter-species differences}

1243 For tissue inter-species analysis, in order to get more accurate comparisons, we 1244 specifically chose three tissues with snRNA-seq data, namely kidney, neocortex and 1245 heart, and processed the raw sequencing data using our pipeline described below in the 1246 'Sc/snRNA-seq data processing' section. Kidney ${ }^{43,44}$, neocortex ${ }^{46}$ and heart ${ }^{49,50}$ data 
1247 were downloaded from NCBI Gene expression omnibus (human kidney: GSE121862,

1248 mouse kidney: GSE119531, human neocortex: GSE97942, human heart: ERP123138,

1249 mouse heart: E-MTAB-7869). For each tissue we preprocessed the UMI matrix of the

1250 three species following three steps: 1 . only orthologs genes among three species were

1251 kept. 2. only genes expressed in at least one cell in one species were kept. 3. the gene

1252 names of the human and mouse UMI matrix were converted into orthologs in Macaca

1253 fascicularis. After preprocessing, the UMI matrices of the three species were integrated

1254 together and the clustering was performed following the standard integrated pipeline

1255 using Seurat (V3.2.2) with the addition of one additional criterion for which only cells

1256 expressing more than 500 genes were kept. Also, we downsampled the cells of human

1257 and macaque neocortex to 10,000 to get a better clustering result. The Seurat clusters

1258 were then annotated into different cell types using cell type-specific markers as

1259 described above. In addition, for the comparison presented in Extended Data Figure 35

1260 we retrieved the publicly available single-cell data for gallbladder, liver and lung from

1261 GEO GSE134355², GEO GSE108098 ${ }^{6}$ and GSE124395 ${ }^{96}$, respectively. Data from the

1262 three species were integrated, clustered and annotated in the same way as described.

\section{Common cell analysis}

1265 We performed common cell analysis for 7 cell types across all the 43 tissues, those

1266 being stromal cells, macrophages/microglia, endothelial cells, smooth muscle cells,

1267 skeletal muscle cells, mesothelial cells and adipocytes. For each cell type, we extracted

1268 those cells from all tissues in our dataset according to the cell type annotation presented

1269 in Extended Data Figure 7-10. For the downstream analysis, we excluded cell types

1270 with numbers lower than 200. Data from different replicates were integrated following

1271 the standard integrated pipeline using Seurat (V3.2.2).

1272

1273 Single-cell trajectory analysis 
1274 Cell lineage trajectory was inferred using Monocle $2^{97}$ following the tutorial. We used

1275 the "differentialGeneTest" function to derive DEG from each cluster and genes

1276 with $q<0.01$ were used to order the cells in a pseudotime analysis. After the cell 1277 trajectories was constructed, DDRtree was used to visualize it in a two-dimensional 1278 space.

1280 Cell-cell interaction network

1281 To assess the cellular crosstalk between different cell types in each tissue, we used 1282 CellPhoneDB, a public repository of ligand-receptor interactions ${ }^{98}$. Cell type-specific 1283 receptor-ligand interactions between cell types were identified based on specific 1284 expression of a receptor by one cell type and a ligand by another cell type. The 1285 interaction score refers to the mean total of all individual ligand-receptor partner 1286 average expression values in the corresponding interacting pairs of cell types. For this 1287 analysis, we applied a statistical method to ensure that only receptors or ligands 1288 expressed in more than $10 \%$ of the cells in the given cluster were considered. The total 1289 mean of the individual partner average expression values in the corresponding 1290 interacting pairs of cell types was calculated. For the cell-cell interaction analysis in 1291 Extended Data Figure 27-31, we plot the figure based on the indicated genes related to $1292 \quad L G R 5$ and LGR6.

1294 Association of GWAS summary data of human diseases and traits with monkey 1295 cell types

1296 To test for the enrichment of human diseases and traits in DEG for each cluster of cells 1297 based on global clustering, we applied LD (linkage disequilibrium) score regression 1298 analysis. For this, we only considered genes with an adjusted $P<0.05$ and fold-change $>$ 12992 in the tested cell types. For accuracy, cell types identified in a number lower than 100 1300 were excluded from this analysis. We converted the gene coordinates of Macaca 1301 fascicularis into hg19 genome coordinates by downloading from Ensembl the 
1302 homologous gene list. Single nucleotide polymorphisms located in gene regions of the

1303 most specific genes in each cell type were added to the baseline model independently

1304 for each cell type (one file for each cell type). We then selected the coefficient $z$-score

$1305 P$ value as a measure of the association of the cell type with the traits. All plots show

1306 the $-\log _{10} P$ value of partitioned LDscore regression.

1308 ScATAC-seq data processing, clustering and cell type identification

1309 Raw sequencing reads from BGISEQ-500 were filtered and demultiplexed using PISA

1310 (version 0.2) (https://github.com/shiquan/PISA). The fragment file of each scATAC-

1311 seq library was used for downstream analysis. TSS (transcription start site) enrichment

1312 score and fragment number of each nuclei was calculated by using ArchR software ${ }^{99}$.

1313 Nuclei with TSS enrichment score lower than five and fragment number lower than

13141,000 were removed. Then, we calculated the doublet score with addDoubletScores

1315 function in ArchR package and filtered doublets by filterDoublets function with

1316 parameter filterRatio $=2$. ScATAC - seq clustering analysis was performed using ArchR

1317 software by first identifying a robust set of peak regions followed by iterative LSI

1318 (latent semantic indexing) clustering. Briefly, we created $500 \mathrm{bp}$ windows tiled across

1319 the genome and determined whether each cell was accessible within each window. Next,

1320 we performed an LSI dimensionality reduction on these windows with addIterativeLSI

1321 function in ArchR packages. We then performed Seurat clustering (FindClusters) on

1322 the LSI dimensions at resolutions of 0.8. Anchors between scATAC-seq and

1323 sc/snRNA-seq datasets were identified and used to transfer cell type labels identified

1324 from the sc/snRNA-seq data. We embedded the data by the TransferData function of

1325 Seurat (version 3.2.2).

\section{Transcription factor motif enrichment analysis}

1328 To predict the motif footprint in peaks within the ACE2 promoter and enhancer 1329 sequences, we extracted genome sequences in the peak region with Seqkit (version 
$1330 \quad 0.7 .0)^{100}$. The sequences were imported into $\mathrm{R}$ and were matched with all Homo sapiens

1331 motifs form JASPAR2018 using matchMotifs function in motifmatchr packages 1332 version 1.8 .0 with default parameter.

\section{Data availability}

1335 All raw data have been deposited to CNGB Nucleotide Sequence Archive (accession code: CNP0001469; https://db.cngb.org/cnsa/project/CNP0001469/reviewlink/).

\section{ACKNOWLEDGMENTS}

1340 We would like to thank Wei Liu and Liangzhi Xu from Huazhen Laboratory Animal

1341 Breeding Centre for helping in the collection of monkey tissues, Dahai Zhu and $\mathrm{Hu} \mathrm{Li}$

1342 from Bioland Laboratory (Guangzhou Regenerative Medicine and Health Guangdong

1343 Laboratory) for technical help, Guoji Guo and Huiyu Sun from Zhejiang University for

1344 providing analytical advice, Guoyi Dong and Chao Liu from BGI research, Xiao Zhang,

1345 Peng Li, and Chen Qi from the Guangzhou Institutes of Biomedicine and Health for

1346 experimental advice. This work was supported by the Shenzhen Key Laboratory of

1347 Single-Cell Omics (ZDSYS20190902093613831), Shenzhen Bay Laboratory

1348 (SZBL2019062801012) and the Guangdong Provincial Key Laboratory of Genome

1349 Read and Write (2017B030301011). Additionally, Longqi Liu was supported by the

1350 National Natural Science Foundation of China (31900466) and Shenzhen Basic

1351 Research Project for Excellent Young Scholars (2020251518), Miguel A. Esteban's was

1352 supported by a Changbai Mountain Scholar award (419020201252), the Strategic

1353 Priority Research Program of the Chinese Academy of Sciences (XDA16030502), and

1354 a Chinese Academy of Sciences-Japan Society for the Promotion of Science joint

1355 research project (GJHZ2093), Giacomo Volpe was supported by a Chinese Academy

1356 of Sciences President's International Fellowship for Foreign Experts (2020FSB0002), 
1357

1358

1359

1360

1361

1362

1363

1364

1365

1366

1367

1368

1369

1370

1371

1372

1373

1374

1375

1376

1377

1378

1379

1380

1381

1382

1383

1384

and Mingyuan Liu was supported by the National Key Research and Development Program of China (2021YFC2600200).

\section{AUTHOR CONTRIBUTIONS}

L.H., Y.H, X.X., M.A.E. and L.L. conceived the idea; Y.H, X.X., M.A.E. and L.L. supervised the work; L.H., X.W., Y.Y., M.A.E. and L.L designed the experiments; L.H., X.W., G.V., Y.Y., X.Zhang., P.F., P.G., X.L., F.Y., S.S., G.L., J.A., Y.Lei., Y. Lai, M.C., C.W., X.G., S.L. and J.M. collected tissue samples; C.L., G.V., Z.W., Y.Y., X.Zhang., P.F., Q.D., Ya. Liu, Y.Huang, H.L., B.W., M.C., J.X., M.W., C. Wang, Y.Z., Y. Yu, H.Z., Y.W. and S.X. performed the experiments. L.H., X.W., G.V., Z.Z., X. Zou, T.P., Y. Lai, L.W., Q.S., H.Y., Yang Liu, D.X., F.H., Z.Zhu and C.Ward performed data analysis. L.H., X.W., C.L., G.V., Z.Z., X.Zou, Z.Wang, T.P., Y.Yang, J.L. and L.L. prepared the figures. H.Y., X.F.W., F.C., T.Y., W.D. and J.C. prepared the website. Zong.W., Z.P., C.W.W., B.Q, A.S., J.I., L.F., Yan Liu, Z.L., X. Liu, H. Zhang, M.L., Q.S., P.M., N.B., P.M.C., Y.G., J.M., M.U., T.T., S.L., H.Y. and J.W. provided relevant advice and reviewed the manuscript. L.H., G.V., M.A.E. and L.L. wrote the manuscript with input from all authors. All other authors contributed to the work. All authors read and approved the manuscript for submission.

\section{COMPETING INTERESTS}

Employees of BGI have stock holdings in BGI. All other authors declare no competing interests.

\section{REFERENCES}

1 Regev, A. et al. The Human Cell Atlas. Elife 6, doi:10.7554/eLife.27041 (2017). 

The Human Cell Atlas: from vision to reality. Nature 550, 451-453, doi:10.1038/550451a (2017).

13883 Han, X. et al. Construction of a human cell landscape at single-cell level. Nature 581, 303-309, doi:10.1038/s41586-020-2157-4 (2020).

13904 Sun, Y. et al. Single-cell landscape of the ecosystem in early-relapse hepatocellular carcinoma. Cell doi:10.1016/j.cell.2020.11.041 (2021).

5 Segerstolpe, A. et al. Single-Cell Transcriptome Profiling of Human Pancreatic Islets in Health and Type 2 Diabetes. Cell Metab 24, 593-607, doi:10.1016/j.cmet.2016.08.020 (2016).

13966 Han, X. et al. Mapping the Mouse Cell Atlas by Microwell-Seq. Cell 172, 10911107 e1017, doi:10.1016/j.cell.2018.02.001 (2018).

13987 Tabula Muris, C. et al. Single-cell transcriptomics of 20 mouse organs creates 1399 a Tabula Muris. Nature 562, 367-372, doi:10.1038/s41586-018-0590-4 (2018).

14008 Cusanovich, D. A. et al. A Single-Cell Atlas of In Vivo Mammalian Chromatin 1401 Accessibility. Cell 174, 1309-1324 e1318, doi:10.1016/j.cell.2018.06.052 1402 (2018).

14039 Carbone, L. et al. Gibbon genome and the fast karyotype evolution of small apes. Nature 513, 195-201, doi:10.1038/nature13679 (2014).

10 Ezran, C. et al. The Mouse Lemur, a Genetic Model Organism for Primate Biology, Behavior, and Health. Genetics 206, 651-664, doi:10.1534/genetics.116.199448 (2017).

11 Mantini, D. et al. Interspecies activity correlations reveal functional correspondence between monkey and human brain areas. Nat Methods 9, 277282, doi:10.1038/nmeth.1868 (2012).

1412

12 Taylor, K. Clinical veterinarian's perspective of non-human primate (NHP) use in drug safety studies. $J$ Immunotoxicol 7, 114-119, doi:10.1080/15476910903213539 (2010). human viral infections. Nat Rev Immunol 18, 390-404, doi:10.1038/s41577018-0005-7 (2018). Distinct Immune Response Landscapes of COVID-19 and Influenza Patients. Immunity 53, 685-696 e683, doi:10.1016/j.immuni.2020.07.009 (2020).

15 Slyper, M. et al. A single-cell and single-nucleus RNA-Seq toolbox for fresh and frozen human tumors. Nat Med 26, 792-802, doi:10.1038/s41591-0200844-1 (2020).

Habib, N. et al. Massively parallel single-nucleus RNA-seq with DroNc-seq. Nat Methods 14, 955-958, doi:10.1038/nmeth.4407 (2017). 
17 Ding, J. et al. Systematic comparison of single-cell and single-nucleus RNAsequencing methods. Nat Biotechnol 38, 737-746, doi:10.1038/s41587-0200465-8 (2020).

$142818 \quad$ Krausgruber, T. et al. Structural cells are key regulators of organ-specific immune responses. Nature 583, 296-302, doi:10.1038/s41586-020-2424-4 (2020).

21 Petrany, M. J. et al. Single-nucleus RNA-seq identifies transcriptional

$22 \mathrm{Mu}, \mathrm{L}$. \& Sanders, I. Human tongue neuroanatomy: Nerve supply and motor

23 Chau, Y. Y. et al. Visceral and subcutaneous fat have different origins and

24 Vijay, J. et al. Single-cell analysis of human adipose tissue identifies depot and

25 Sun, W. et al. snRNA-seq reveals a subpopulation of adipocytes that regulates

27 Kretzschmar, K. et al. Profiling proliferative cells and their progeny in damaged murine hearts. Proc Natl Acad Sci $U$ S A

115, E12245-E12254, doi:10.1073/pnas.1805829115 (2018). of fat grafts via crosstalk between the Nrf2 and TLR4 pathways. Cell Death Dis 7, e2369, doi:10.1038/cddis.2016.261 (2016). Ha, C. W. Y. et al. Translocation of Viable Gut Microbiota to Mesenteric Adipose Drives Formation of Creeping Fat in Humans. Cell 183, 666-683 e617, doi:10.1016/j.cell.2020.09.009 (2020).

Adler, E., Mhawech-Fauceglia, P., Gayther, S. A. \& Lawrenson, K. PAX8 expression in ovarian surface epithelial cells. Hum Pathol 46, 948-956, doi:10.1016/j.humpath.2015.03.017 (2015).

$\mathrm{Ng}$, A. et al. Lgr5 marks stem/progenitor cells in ovary and tubal epithelia. Nat Cell Biol 16, 745-757, doi:10.1038/ncb3000 (2014). 
Hagai, T. et al. Gene expression variability across cells and species shapes innate immunity. Nature 563, 197-202, doi:10.1038/s41586-018-0657-2 (2018).

146833 Nusse, R. \& Clevers, H. Wnt/ $\beta$-Catenin Signaling, Disease, and Emerging Therapeutic Modalities. Cell 169, 985-999, doi:10.1016/j.cell.2017.05.016 (2017).

$147134 \quad$ Clevers, H. \& Nusse, R. Wnt/ $\beta$-catenin signaling and disease. Cell 149, $1192-$ 1205, doi:10.1016/j.cell.2012.05.012 (2012). doi:10.1038/cr.2008.47 (2008). Leung, C., Tan, S. H. \& Barker, N. Recent Advances in Lgr5(+) Stem Cell Research. Trends Cell Biol 28, 380-391, doi:10.1016/j.tcb.2018.01.010 (2018). Kong, Y. et al. LGR6 Promotes Tumor Proliferation and Metastasis through Wnt/ $\beta$-Catenin Signaling in Triple-Negative Breast Cancer. Mol Ther Oncolytics 18, 351-359, doi:10.1016/j.omto.2020.06.020 (2020). Huch, M. et al. In vitro expansion of single Lgr5+ liver stem cells induced by (2013).

Barker, N. et al. Identification of stem cells in small intestine and colon by marker gene Lgr5. Nature 449, 1003-1007, doi:10.1038/nature06196 (2007). Chen, M. et al. $\operatorname{Lgr} 5(+)$ amacrine cells possess regenerative potential in the retina of adult mice. Aging Cell 14, 635-643, doi:10.1111/acel.12346 (2015). Aguilar, A. Development: Pax2 keeps nephron progenitors on track. Nat Rev Nephrol 13, 444, doi:10.1038/nrneph.2017.87 (2017).

Schutgens, F. et al. Troy/TNFRSF19 marks epithelial progenitor cells during mouse kidney development that continue to contribute to turnover in adult kidney. Proc Natl Acad Sci U S A 114, E11190-E11198, doi:10.1073/pnas.1714145115 (2017).

Lake, B. B. et al. A single-nucleus RNA-sequencing pipeline to decipher the molecular anatomy and pathophysiology of human kidneys. Nat Commun 10, 2832, doi:10.1038/s41467-019-10861-2 (2019). Nucleus over Single-Cell RNA Sequencing of Adult Kidney: Rare Cell Types and Novel Cell States Revealed in Fibrosis. J Am Soc Nephrol 30, 23-32, doi:10.1681/ASN.2018090912 (2019).

5 Barker, N. et al. Lgr5(+ve) stem/progenitor cells contribute to nephron formation during kidney development. Cell Rep 2, 540-552, doi:10.1016/j.celrep.2012.08.018 (2012).

6 Lake, B. B. et al. Integrative single-cell analysis of transcriptional and epigenetic states in the human adult brain. Nat Biotechnol 36, 70-80, doi:10.1038/nbt.4038 (2018). 
47 Nakashima, H. et al. R-spondin 2 promotes acetylcholine receptor clustering at the neuromuscular junction via Lgr5. Sci Rep 6, 28512, doi:10.1038/srep28512 (2016).

1509

48 Leung, C. et al. Lgr5 Marks Adult Progenitor Cells Contributing to Skeletal Muscle Regeneration and Sarcoma Formation. Cell Rep 33, 108535, doi:10.1016/j.celrep.2020.108535 (2020).

1512

49 Litvinukova, M. et al. Cells of the adult human heart. Nature 588, 466-472, doi:10.1038/s41586-020-2797-4 (2020).

1514

Vidal, R. et al. Transcriptional heterogeneity of fibroblasts is a hallmark of the aging heart. JCI Insight 4, doi:10.1172/jci.insight.131092 (2019).

1516 Vankelecom, H. Non-hormonal cell types in the pituitary candidating for stem

18, 559-570, doi:10.1016/j.semcdb.2007.04.006 (2007).

Klein, D. et al. Wnt2 acts as a cell type-specific, autocrine growth factor in rat hepatic sinusoidal endothelial cells cross-stimulating the VEGF pathway. Hepatology 47, 1018-1031, doi:10.1002/hep.22084 (2008).

Karner, C. M. et al. Canonical Wnt9b signaling balances progenitor cell expansion and differentiation during kidney development. Development 138, 1247-1257, doi:10.1242/dev.057646 (2011).

Niehrs, C. The complex world of WNT receptor signalling. Nat Rev Mol Cell Biol 13, 767-779, doi:10.1038/nrm3470 (2012).

Zhang, M. et al. $\beta$-Catenin safeguards the ground state of mouse pluripotency by strengthening the robustness of the transcriptional apparatus. Sci Adv 6, eaba1593, doi:10.1126/sciadv.aba1593 (2020). pathogenesis, diagnosis, and management. Lancet Infect Dis 18, e1-e13, doi:10.1016/S1473-3099(17)30398-5 (2018). with the Human Cell Atlas. Nat Rev Mol Cell Biol 21, 415-416, doi:10.1038/s41580-020-0267-3 (2020). and CD46. Rev Med Virol 14, 217-229, doi:10.1002/rmv.430 (2004).

Zhu, N. et al. A Novel Coronavirus from Patients with Pneumonia in China, 2019. N Engl J Med 382, 727-733, doi:10.1056/NEJMoa2001017 (2020).

Hoffmann, M. et al. SARS-CoV-2 Cell Entry Depends on ACE2 and TMPRSS2 and Is Blocked by a Clinically Proven Protease Inhibitor. Cell 181, 271-280 e278, doi:10.1016/j.cell.2020.02.052 (2020).

Rockx, B. et al. Comparative pathogenesis of COVID-19, MERS, and SARS in a nonhuman primate model. Science 368, 1012-1015, doi:10.1126/science.abb7314 (2020).

Ying, M. et al. COVID-19 with acute cholecystitis: a case report. BMC Infect Dis 20, 437, doi:10.1186/s12879-020-05164-7 (2020). 
Ziegler, C. G. K. et al. SARS-CoV-2 Receptor ACE2 Is an InterferonStimulated Gene in Human Airway Epithelial Cells and Is Detected in Specific Cell Subsets across Tissues. Cell 181, 1016-1035 e1019, doi:10.1016/j.cell.2020.04.035 (2020).

155264 Chua, R. L. et al. COVID-19 severity correlates with airway epitheliumimmune cell interactions identified by single-cell analysis. Nat Biotechnol 38, 970-979, doi:10.1038/s41587-020-0602-4 (2020).

65 Tosi, M. F. Innate immune responses to infection. J Allergy Clin Immunol 116, 241-249; quiz 250, doi:10.1016/j.jaci.2005.05.036 (2005). IL-6 activity in COVID-19. doi:10.1016/j.isci.2020.101896 (2021).

Shen, B. et al. Proteomic and Metabolomic Characterization of COVID-19 Patient Sera. Cell 182, 59-72 e15, doi:10.1016/j.cell.2020.05.032 (2020).

1562

Nie, X. et al. Multi-organ proteomic landscape of COVID-19 autopsies. Cell 184, 775-791 e714, doi:10.1016/j.cell.2021.01.004 (2021).

1564

69 Gate, D. et al. Clonally expanded CD8 T cells patrol the cerebrospinal fluid in Alzheimer's disease. Nature 577, 399-404, doi:10.1038/s41586-019-1895-7 (2020). responses in parents of children with attention deficit hyperactivity disorder. Psychiatr Genet 30, 65-72, doi:10.1097/YPG.0000000000000252 (2020).

Ghaoui, R. et al. Mutations in HSPB8 causing a new phenotype of distal myopathy and motor neuropathy. Neurology 86, 391-398, doi:10.1212/WNL.0000000000002324 (2016). Tse, K. H. \& Herrup, K. DNA damage in the oligodendrocyte lineage and its role in brain aging. Mech Ageing Dev 161, 37-50, doi:10.1016/j.mad.2016.05.006 (2017). Wang, S. et al. Single-Cell Transcriptomic Atlas of Primate Ovarian Aging. Cell 180, 585-600 e519, doi:10.1016/j.cell.2020.01.009 (2020).

75 Khrameeva, E. et al. Single-cell-resolution transcriptome map of human, chimpanzee, bonobo, and macaque brains. Genome Res 30, 776-789, doi:10.1101/gr.256958.119 (2020).

76 Stray, L. L. et al. Motor regulation problems and pain in adults diagnosed with ADHD. Behav Brain Funct 9, 18, doi:10.1186/1744-9081-9-18 (2013). problems in ADHD identifies genes for brain and muscle function. World J Biol Psychiatry 13, 211-222, doi:10.3109/15622975.2011.560279 (2012). 

Villiger, P. M. et al. Tocilizumab for induction and maintenance of remission in giant cell arteritis: a phase 2, randomised, double-blind, placebo-controlled trial. Lancet 387, 1921-1927, doi:10.1016/S0140-6736(16)00560-2 (2016).

79 Marcheque, J., Bussolati, B., Csete, M. \& Perin, L. Concise Reviews: Stem Cells and Kidney Regeneration: An Update. Stem Cells Transl Med 8, 82-92, doi:10.1002/sctm.18-0115 (2019).

80 Little, M. H. \& Lawlor, K. T. Recreating, expanding and using nephron progenitor populations. Nat Rev Nephrol 16, 75-76, doi:10.1038/s41581-0190238-0 (2020).

81 Chavali, M. et al. Wnt-Dependent Oligodendroglial-Endothelial Interactions Regulate White Matter Vascularization and Attenuate Injury. Neuron 108, 1130-1145 e1135, doi:10.1016/j.neuron.2020.09.033 (2020).

82 Girardi, F. \& Le Grand, F. Wnt Signaling in Skeletal Muscle Development and Regeneration. Prog Mol Biol Transl Sci 153, 157-179, doi:10.1016/bs.pmbts.2017.11.026 (2018).

83 Heallen, T. et al. Hippo pathway inhibits Wnt signaling to restrain cardiomyocyte proliferation and heart size. Science 332, 458-461, doi:10.1126/science.1199010 (2011).

84 Osmundsen, A. M., Keisler, J. L., Taketo, M. M. \& Davis, S. W. Canonical WNT Signaling Regulates the Pituitary Organizer and Pituitary Gland Formation. Endocrinology 158, 3339-3353, doi:10.1210/en.2017-00581 (2017).

85 Chen, A. et al. Large field of view-spatially resolved transcriptomics at nanoscale resolution. bioRxiv, 2021.2001.2017.427004, doi:10.1101/2021.01.17.427004 (2021).

86 Mitchell, J. F. \& Leopold, D. A. The marmoset monkey as a model for visual neuroscience. Neurosci Res 93, 20-46, doi:10.1016/j.neures.2015.01.008 (2015).

87 Bakken, T. E. et al. Single-nucleus and single-cell transcriptomes compared in matched cortical cell types. PLoS One 13, e0209648, doi:10.1371/journal.pone.0209648 (2018).

88 Laukoter, S. et al. Cell-Type Specificity of Genomic Imprinting in Cerebral Cortex. Neuron 107, 1160-1179 e1169, doi:10.1016/j.neuron.2020.06.031 (2020).

89 Dobin, A. et al. STAR: ultrafast universal RNA-seq aligner. Bioinformatics 29, 15-21, doi:10.1093/bioinformatics/bts635 (2013).

90 Tarasov, A., Vilella, A. J., Cuppen, E., Nijman, I. J. \& Prins, P. Sambamba: fast processing of NGS alignment formats. Bioinformatics 31, 2032-2034, doi:10.1093/bioinformatics/btv098 (2015).

91 Del-Aguila, J. L. et al. A single-nuclei RNA sequencing study of Mendelian and sporadic AD in the human brain. Alzheimers Res Ther 11, 71, doi:10.1186/s13195-019-0524-x (2019). 
162992 McGinnis, C. S., Murrow, L. M. \& Gartner, Z. J. DoubletFinder: Doublet 1630 Detection in Single-Cell RNA Sequencing Data Using Artificial Nearest Neighbors. Cell Syst 8, 329-337 e324, doi:10.1016/j.cels.2019.03.003 (2019).

163293 Wolf, F. A., Angerer, P. \& Theis, F. J. SCANPY: large-scale single-cell gene expression data analysis. Genome Biol 19, 15, doi:10.1186/s13059-017-1382-0 (2018).

94 Stuart, T. et al. Comprehensive Integration of Single-Cell Data. Cell 177, 18881902.e1821, doi:10.1016/j.cell.2019.05.031 (2019).

95 Yu, G., Wang, L. G. \& He, Q. Y. ChIPseeker: an R/Bioconductor package for ChIP peak annotation, comparison and visualization. Bioinformatics 31, 23822383, doi:10.1093/bioinformatics/btv145 (2015).

96 Aizarani, N. et al. A human liver cell atlas reveals heterogeneity and epithelial progenitors. Nature 572, 199-204, doi:10.1038/s41586-019-1373-2 (2019).

97 Trapnell, C. et al. The dynamics and regulators of cell fate decisions are revealed by pseudotemporal ordering of single cells. Nat Biotechnol 32, 381386, doi:10.1038/nbt.2859 (2014). CellPhoneDB: inferring cell-cell communication from combined expression of multi-subunit ligand-receptor complexes. Nat Protoc 15, 1484-1506, doi:10.1038/s41596-020-0292-x (2020).

99 Granja, J. M. et al. ArchR is a scalable software package for integrative singlecell chromatin accessibility analysis. Nat Genet, doi:10.1038/s41588-02100790-6 (2021). Toolkit for FASTA/Q File Manipulation. PLoS One 11, e0163962, doi:10.1371/journal.pone.0163962 (2016). 
bioRxiv preprint doi: https://doi.org/10.1101/2021.12.13.472311; this version posted December 13, 2021. The copyright holder for this

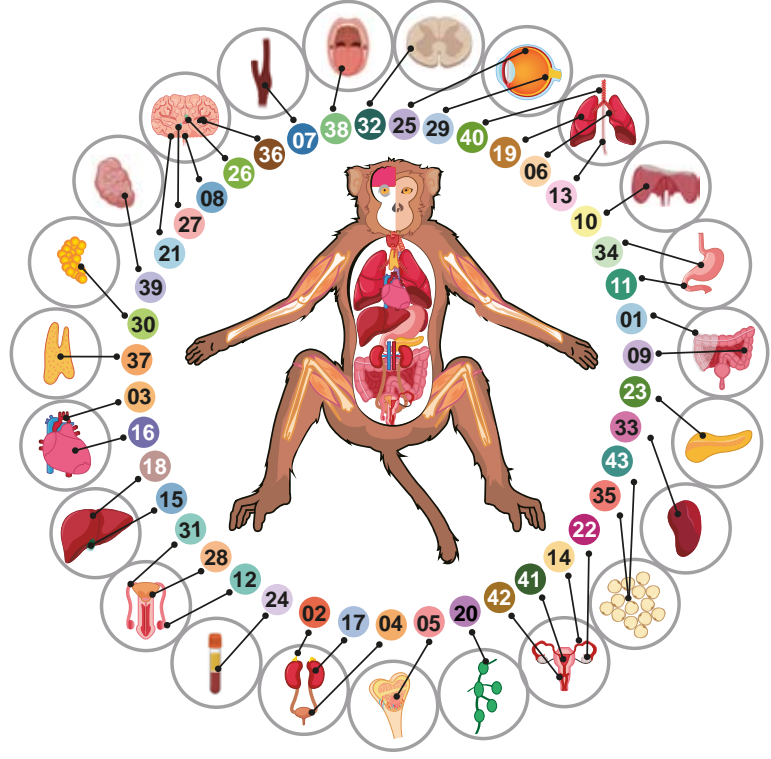

$\begin{array}{llll}01 \text { Abdominal wall } & 06 \text { Bronchus } & \text { (11) Duodenum } & 16 \text { Heart } \\ 02 \text { Adrenal gland } & 07 \text { Carotid } & 12 \text { Epididymis } & 17 \text { Kidney } \\ 03 \text { Aorta } & 08 \text { Cerebellum } & 13 \text { Esophagus } & 18 \text { Liver } \\ 04 \text { Bladder } & 09 \text { Colon } & 14 \text { Fallopian tube } & 19 \text { Lung } \\ 05 \text { Bone marrow } & 10 \text { Diaphragm } & 15 \text { Gallbladder } & 20 \text { Lymph node }\end{array}$
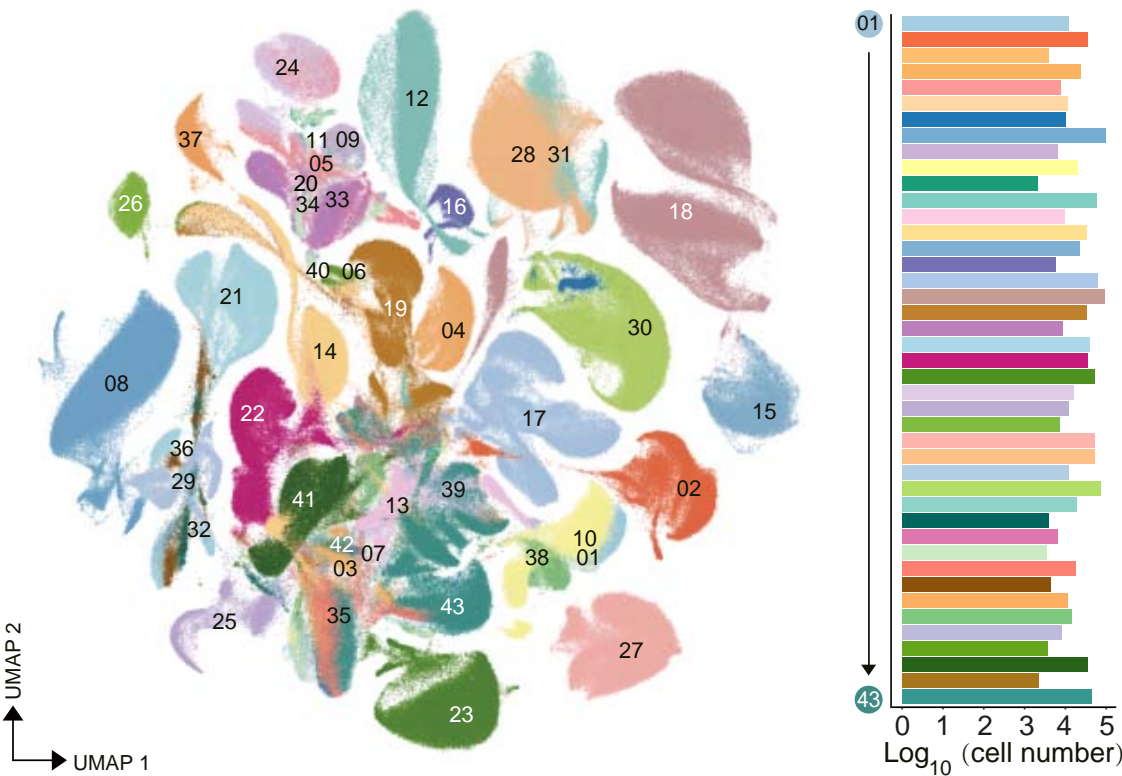

21 Neocortex

22 Ovary

23 Pancreas

24 PBMC

25 Pigmentary epi

\begin{abstract}
26) Pineal gland
27 Pituitary gland

28 Prostate

29 Retina

30 Salivary gland

31 Spermaduct

32 Spinal cord

33 Spleen

34 Stomach
\end{abstract}

35 Subcutaneous adipose

\begin{abstract}
36 Substantia nigra 41 Uterus
37 Thyroid 42 Vagina

38 Tongue

39 Tonsil

(40) Trachea
\end{abstract}

b Immune

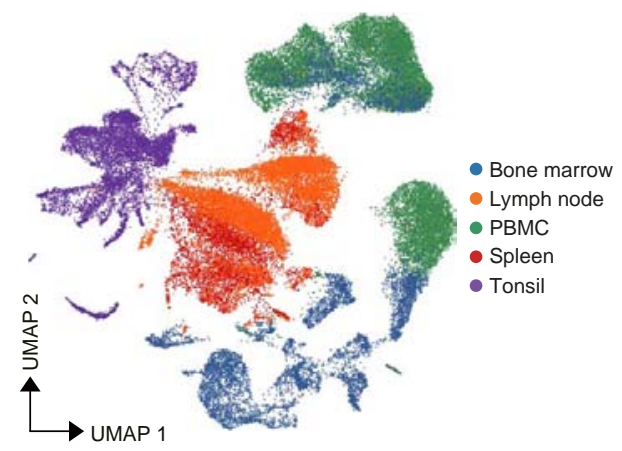

C

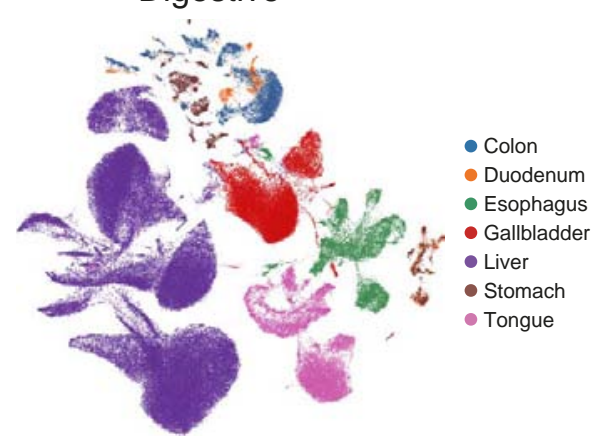

02 Adrenal endothelial cell 3 Adrenal smooth muscle cell Alveolar epithelial type 1 cell Alveolar epithelial type 2 cell 6 Ascending loop of Henle cell Astrocyte

8 B cell 1

B cell 2 adder epithelial cell

Bone marrow lymphoid progenito

2 Bone marrow myeloid progenitor

13 Bone marrow stromal cell

14 Cardiomyocyte

15 Cerebellum astrocyte

16) Cerebellum microglia

Cerebellum oligodendrocyte

Chromaffin cell

19 Club/goblet cell

Connecting tubule cell

1 Corticotrope cell

Diaphragm mesothelial cell

Distal convoluted tubule cell

24 Endothelial cell 1

25 Endothelial cell 2

26 Enteric glial cell

27 Epididymis basal cell
Epididymis smooth muscle cell

29 Epididymis sterociliated cell

29 Epididymis stero

Esophagus skeletal muscle myonucle

32 Esophagus squamous epithelial cell

Esophagus stromal cell

5 Fallopian tube ep

36 Fasciculata cell

Female reproductive ciliated cell

Female reproductive strom cell

Folliculostellate/gonadotrope cell

Gallbladder glandular cell

1 Gallbladder glandular cell/hepatocyte

42 GI tract monocyte

43 GI tract stromal cell

Glomerulosa cell

45 Granule cell

Granulosa cell
Hepatic stellate cell

Hepatic stellate cell

Kidney endothelial cell

Kidney endothelial cell

Lactotrope cell

Liver endothelial ce

3 Macrophage

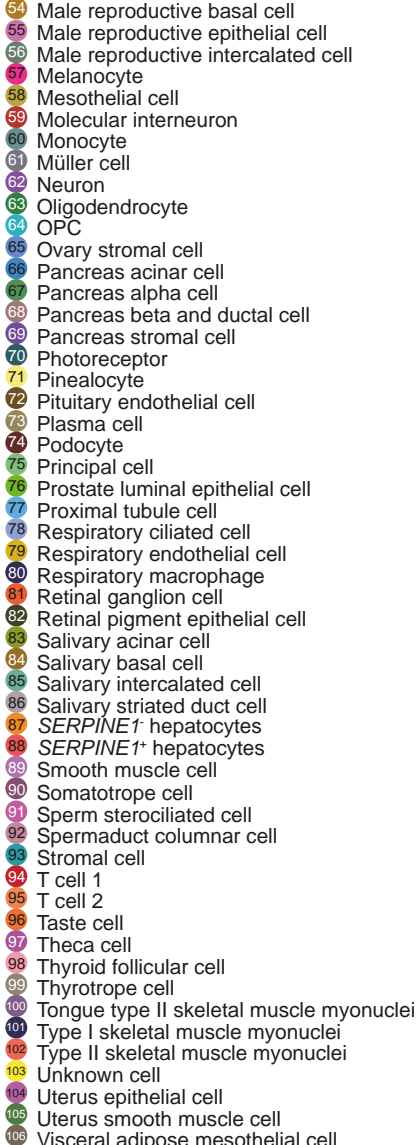

Figure 1 
bioRxiv preprint doi: https://doi.org/10.1101/2021.12.13.472311; this version posted December 13, 2021. The copyright holder for this

Diaphrágm Abdomin

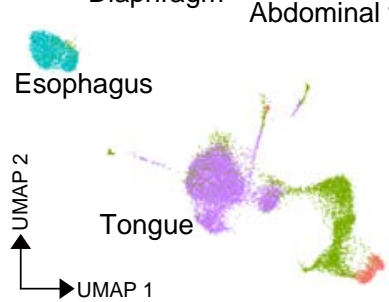

- Abdominal wall Esophagus

- Diaphragm Tongue

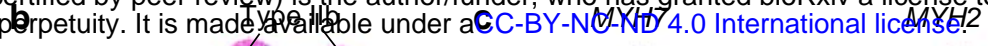

d

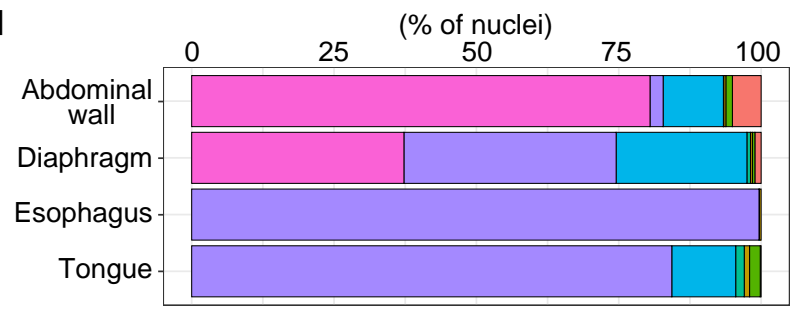

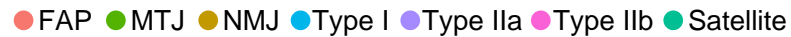
- FAP
- MTJ
- Type I
NMJ
Satellite
- Type Ila
Type Ilb

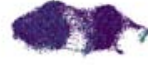

Type lla FAP

MTJ Satellite

\section{Type I}

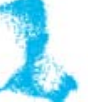

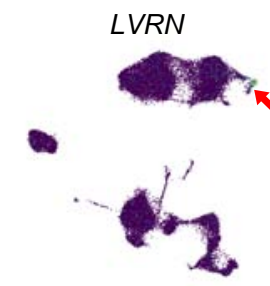

PAX7
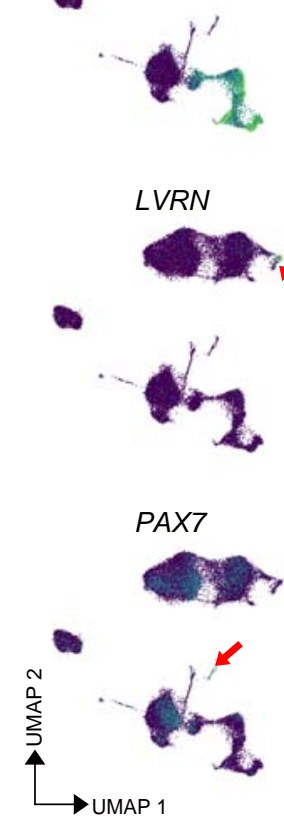

2.
\$

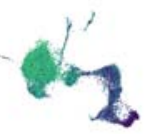

NAV3

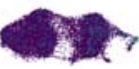

-

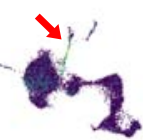

COL22A1

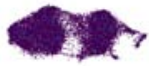

6

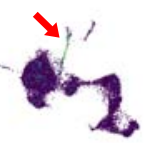

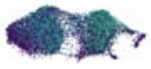

ำ

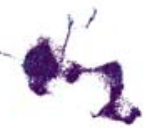

ETV5
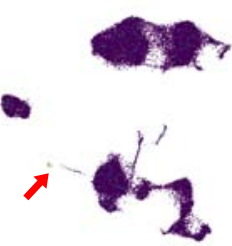

MUSK
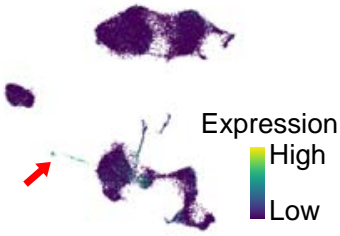

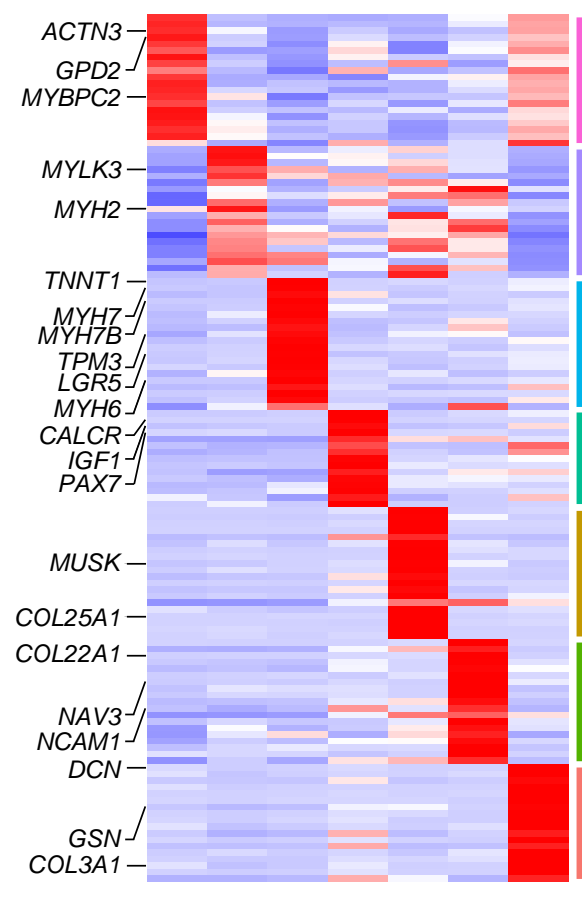

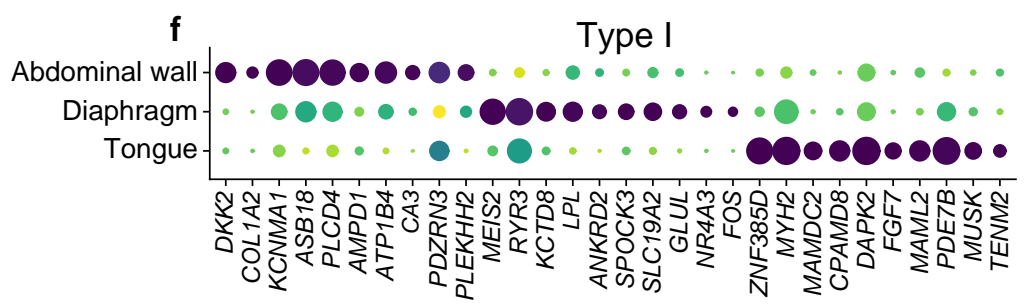

Type I Satellite

NMJ

Type Ila

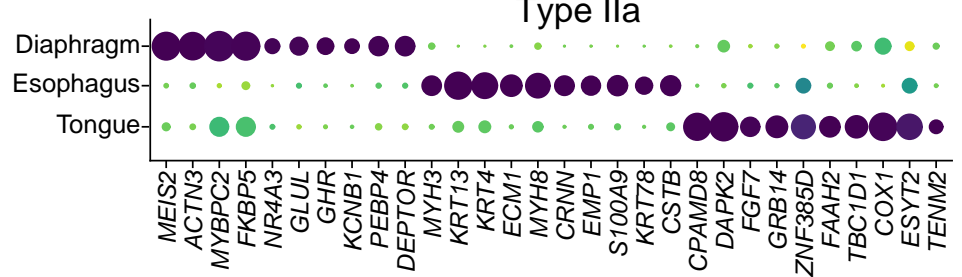

Type IIb g

Mesothelial

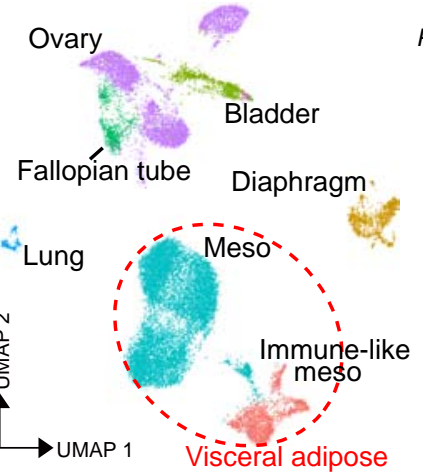

h

Visceral adipose

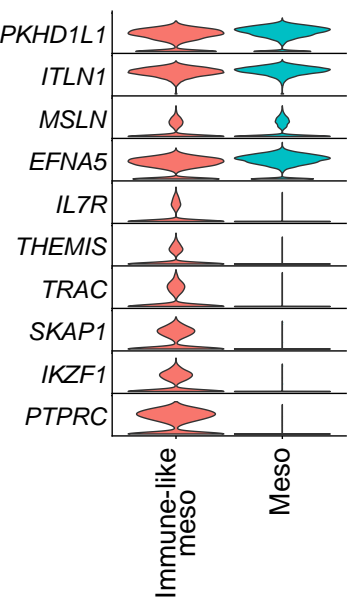

i
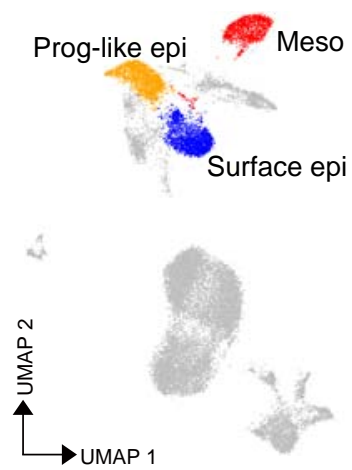

Expression High Ratio (\%)

- 25 ๑50

MTJ

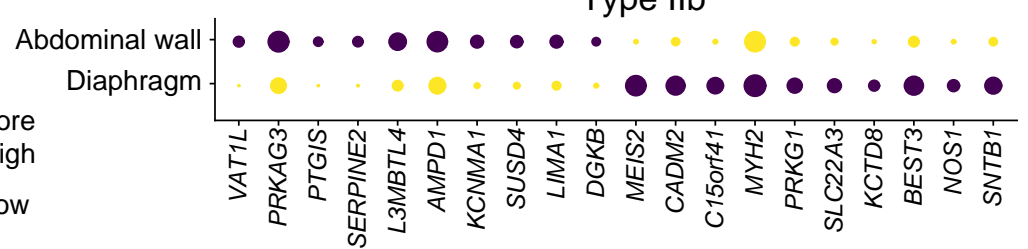

k

j

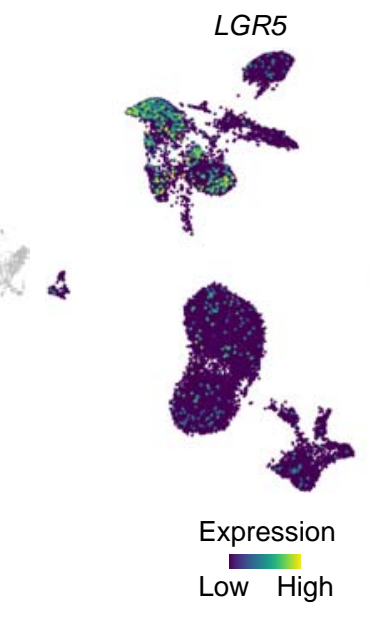

Ovary

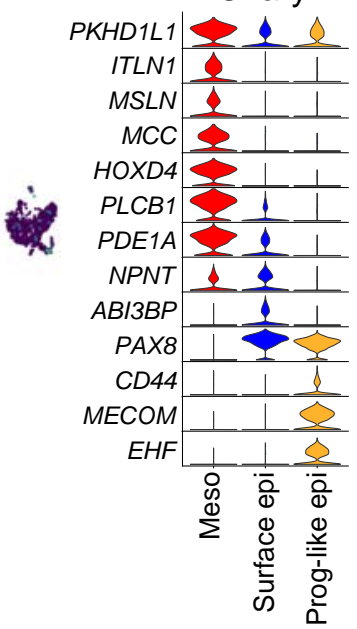

Figure 2 
bioRxiv preprint doi: https://doi.org/10.1101/2021.12.13.472311; this version posted December 13,2021 . The copyright holder for this

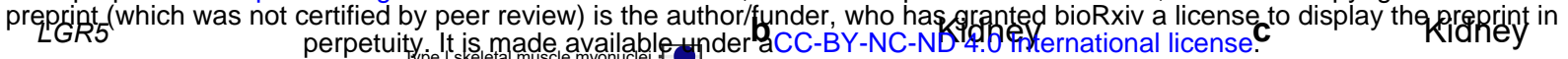
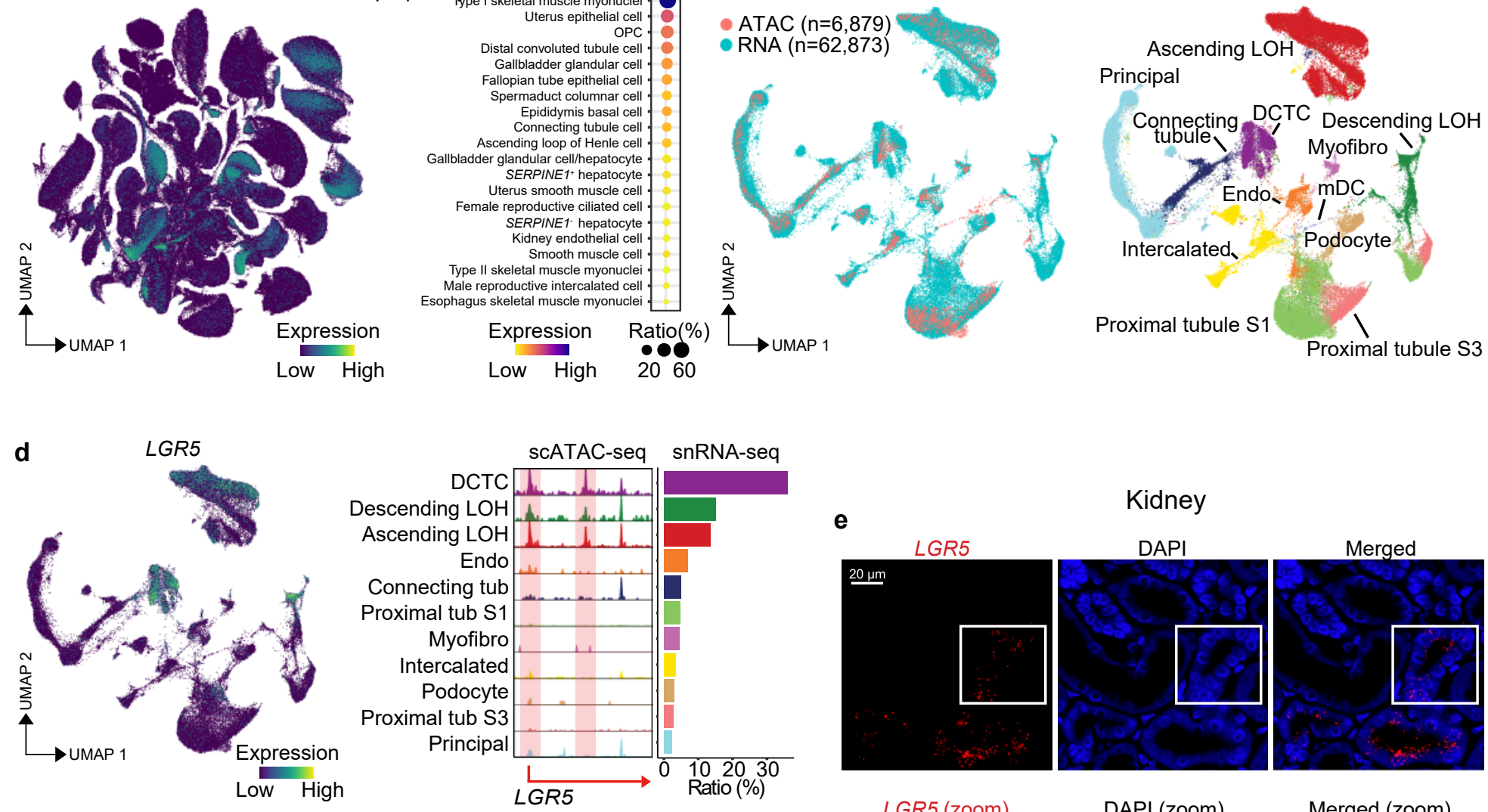

Kidney

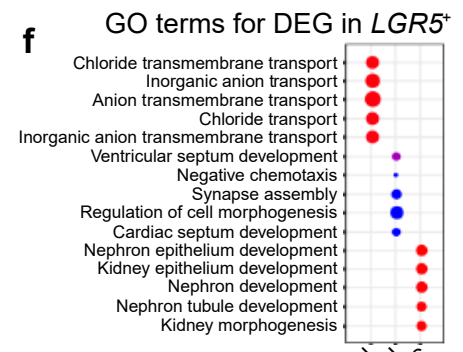

Adjusted $P$ Ratio(\%)

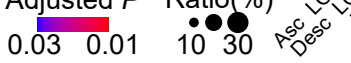

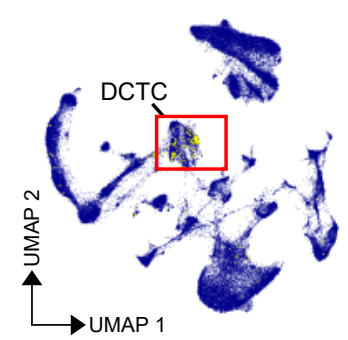

LGR5'TNFRSF19+
LHX1+PAX $2^{+}$

DCTC Connecting tubule Proximal tubule S1 Principal Podocyte Intercalated Endo

Descending LOH

${ }^{0}$ Ratio (\%) ${ }^{2.5}$
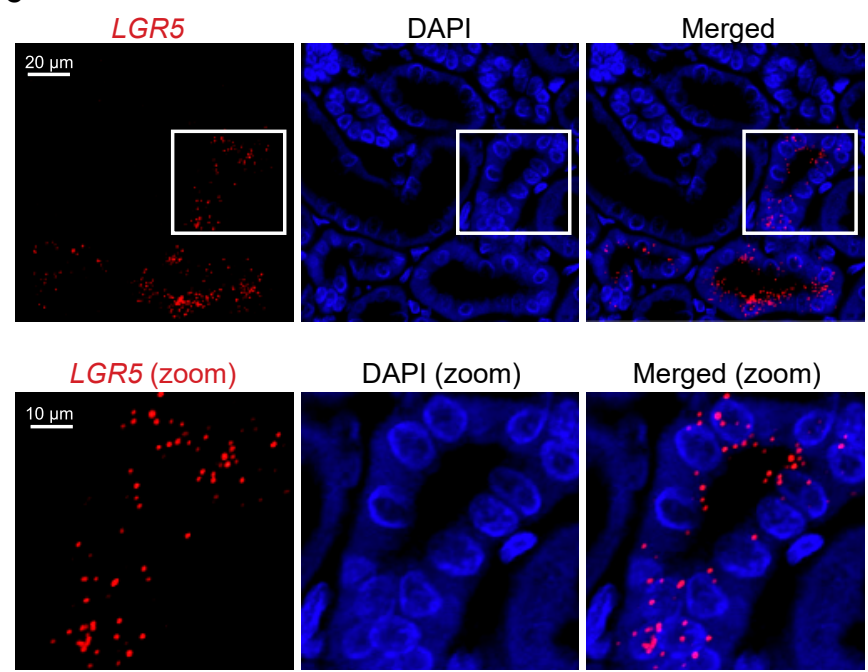

DAPI (zoom)

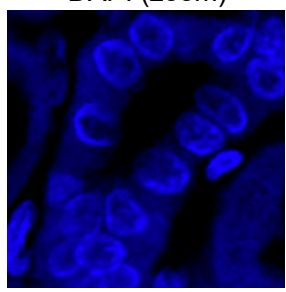

Merged (zoom)

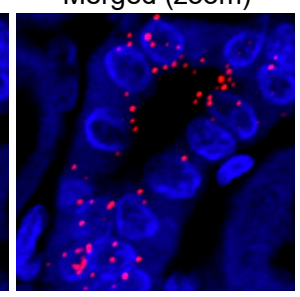

g

Muscle

Skeletal, - - - 'Diaphragm Ābodominal ! 2, Bladder ' Tongue I Esophágus Vagina 'Carotid' 'L', Uterus'

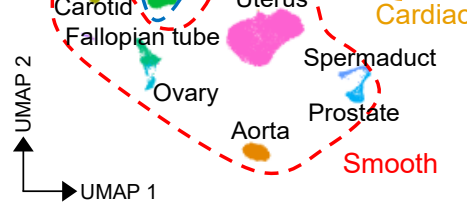

h

LGR5

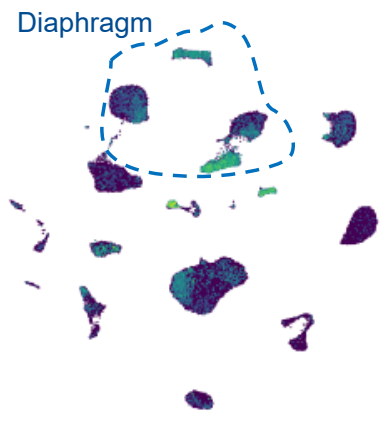

MYH2 (fast-twitch myofibers)

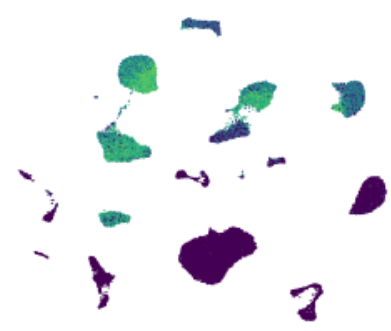

1
MYH7 (slow-twitch myofibers)

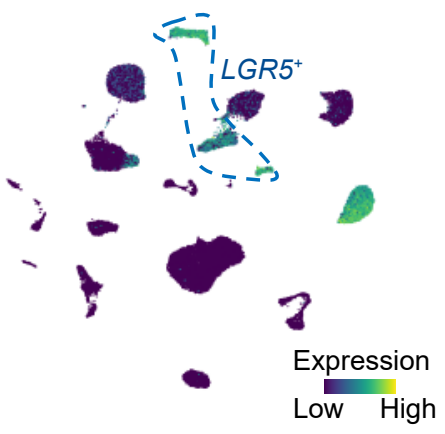

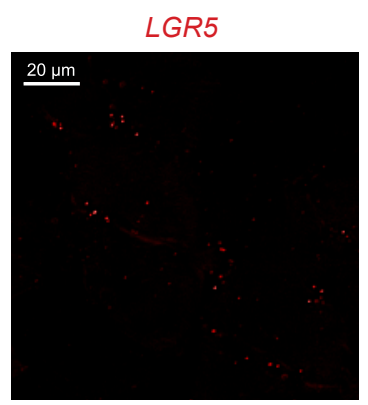
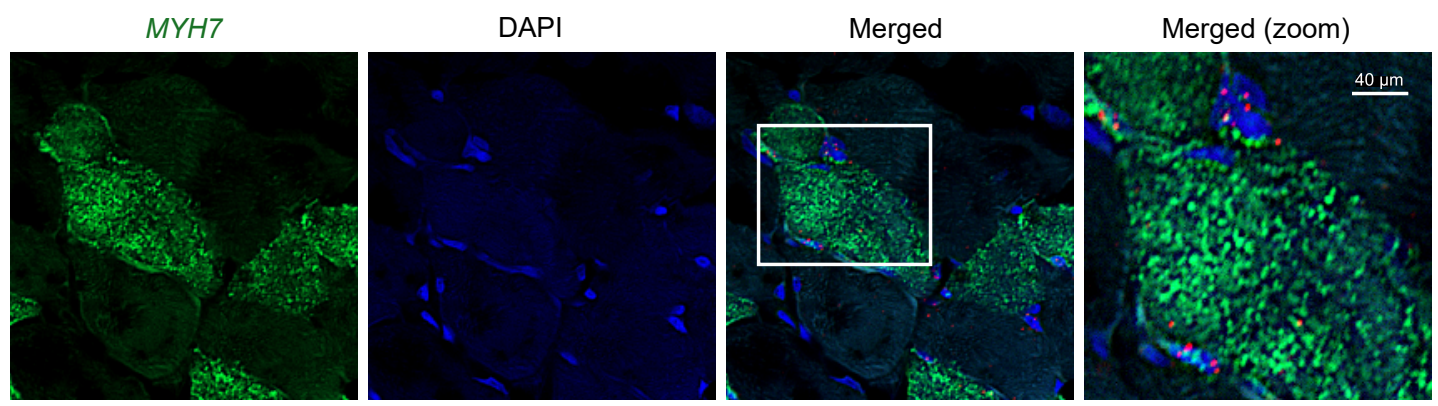


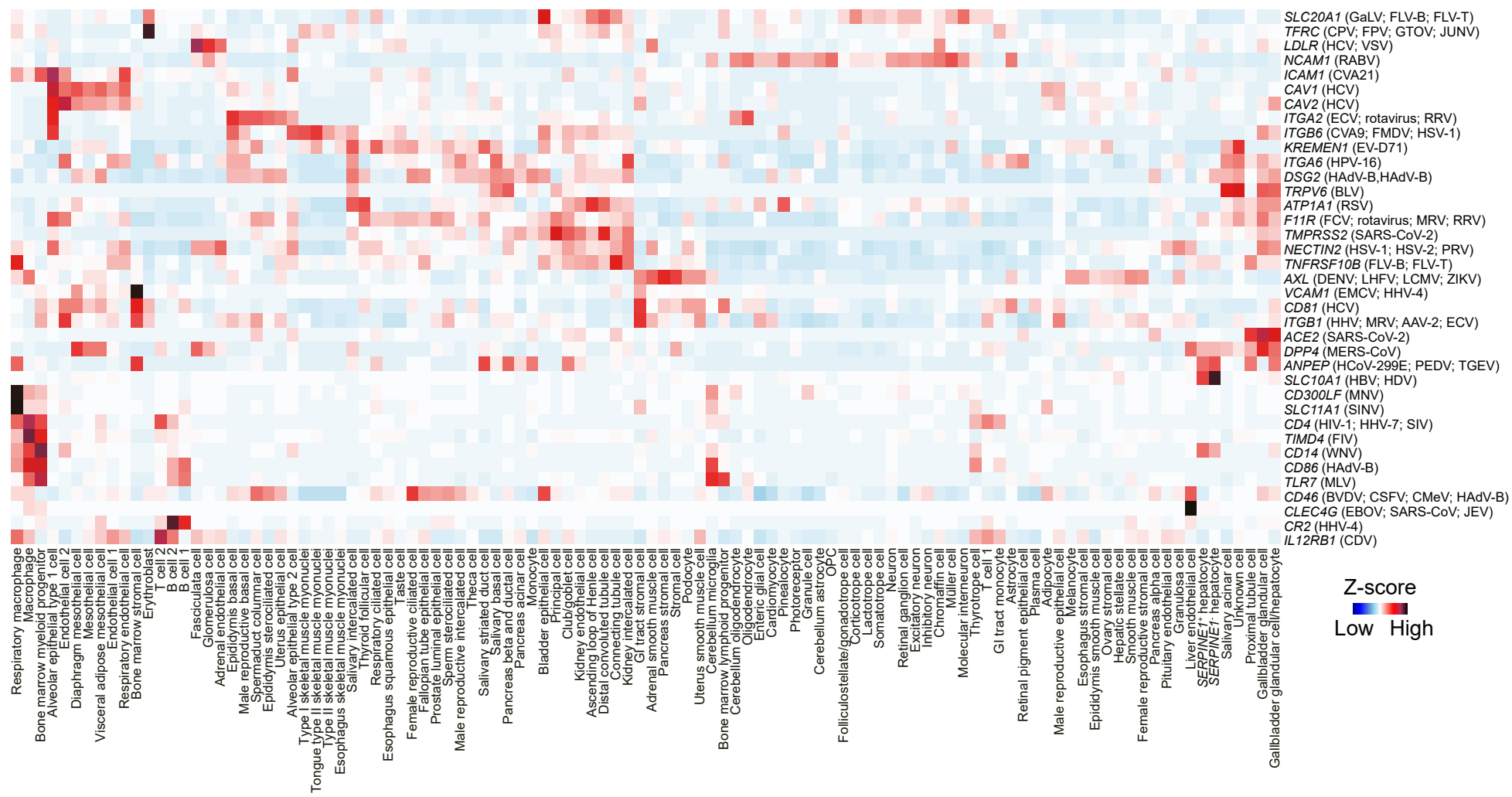

b
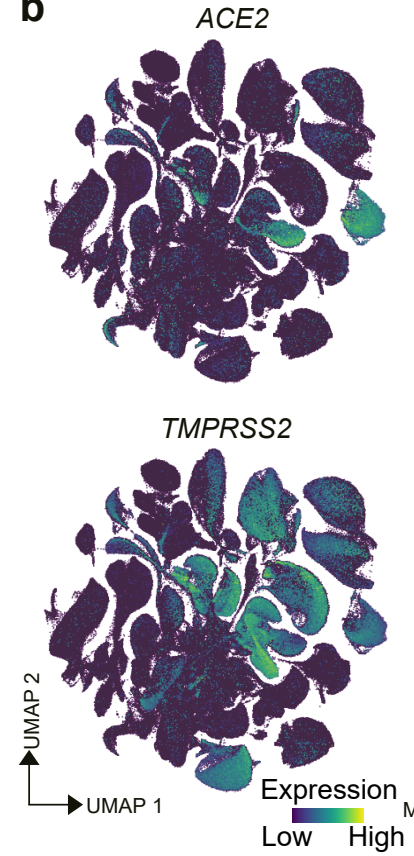

d

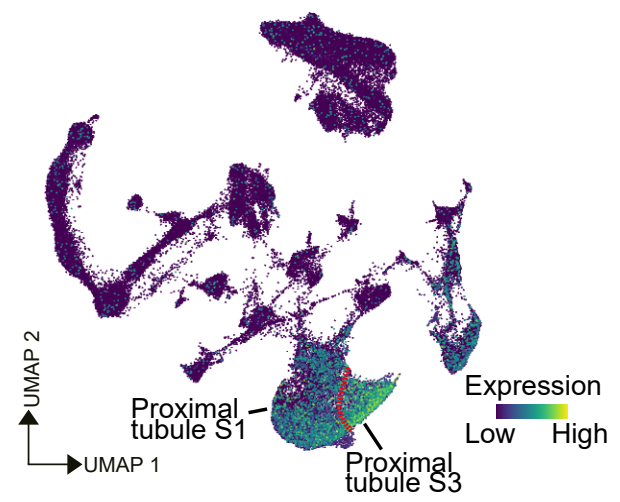

C

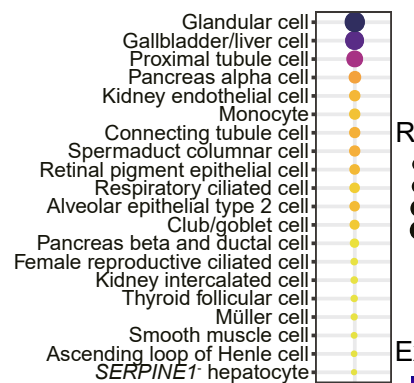

Ratio (\%)
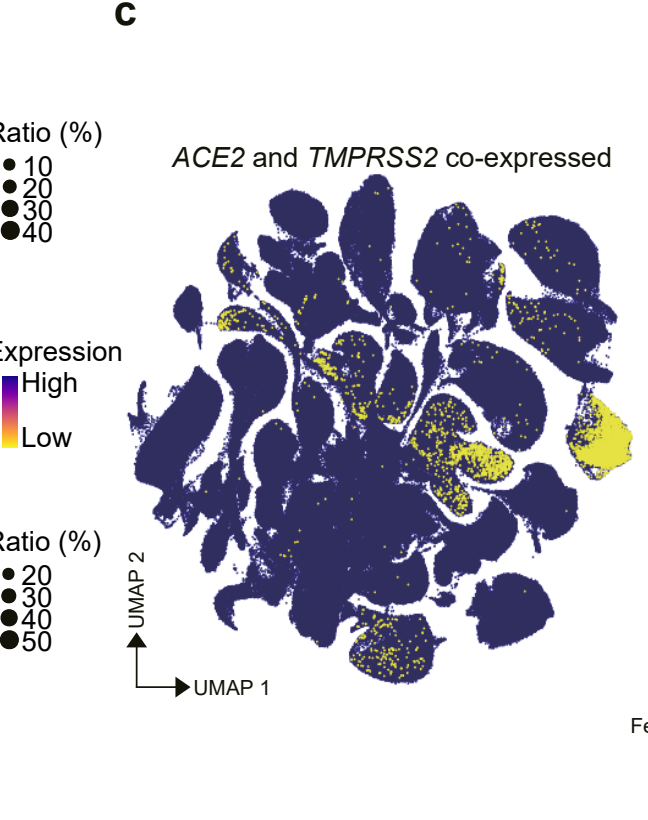

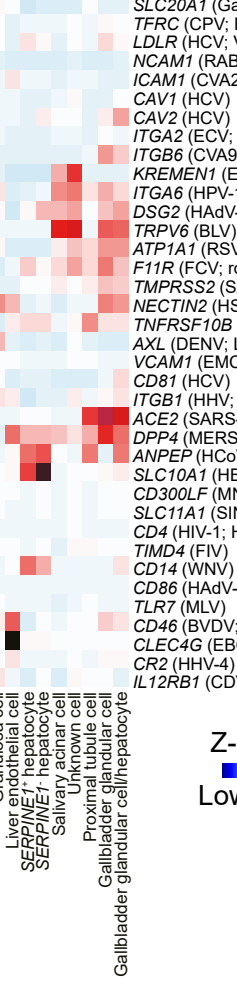

Glandular cell Gallbladder/liver cell Proximal tubule cell

Club/goblet cell Kidney endothelial cell Connecting tubule cell Spermaduct columnar cell Pancreas beta and ductal cell

Respiratory ciliated cell Monocyte Principal cell Alveolar epithelial type 2 cell Kidney intercalated cell Distal convoluted tubule cell Pancreas alpha cell Ascending loop of Henle cell Bladder epithelial cell Pancreas acinar cell Female reproductive ciliated cell Thyroid follicular cell

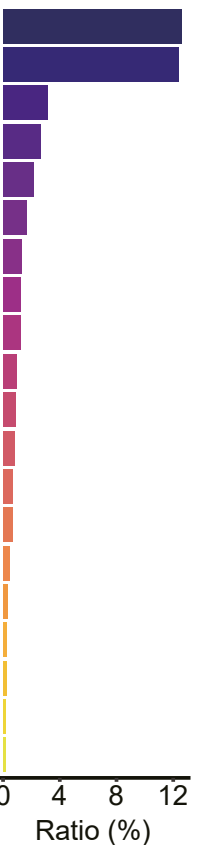

e

IRF1 JUNB FOXA1 IRF4 IRF5 STAT1/3

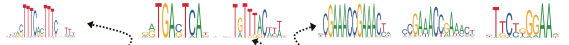
snRNA-seq

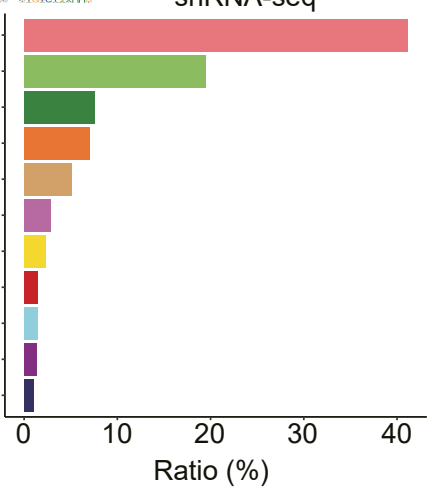


bioRxiv preprint doi: https://doi.org/10.1101/2021.12.13.472311; this version posted December 13, 2021. The copyright holder for this preprint (which was not certified by peer review) is the author/funder, who has granted bioRxiv a license to display the preprint in
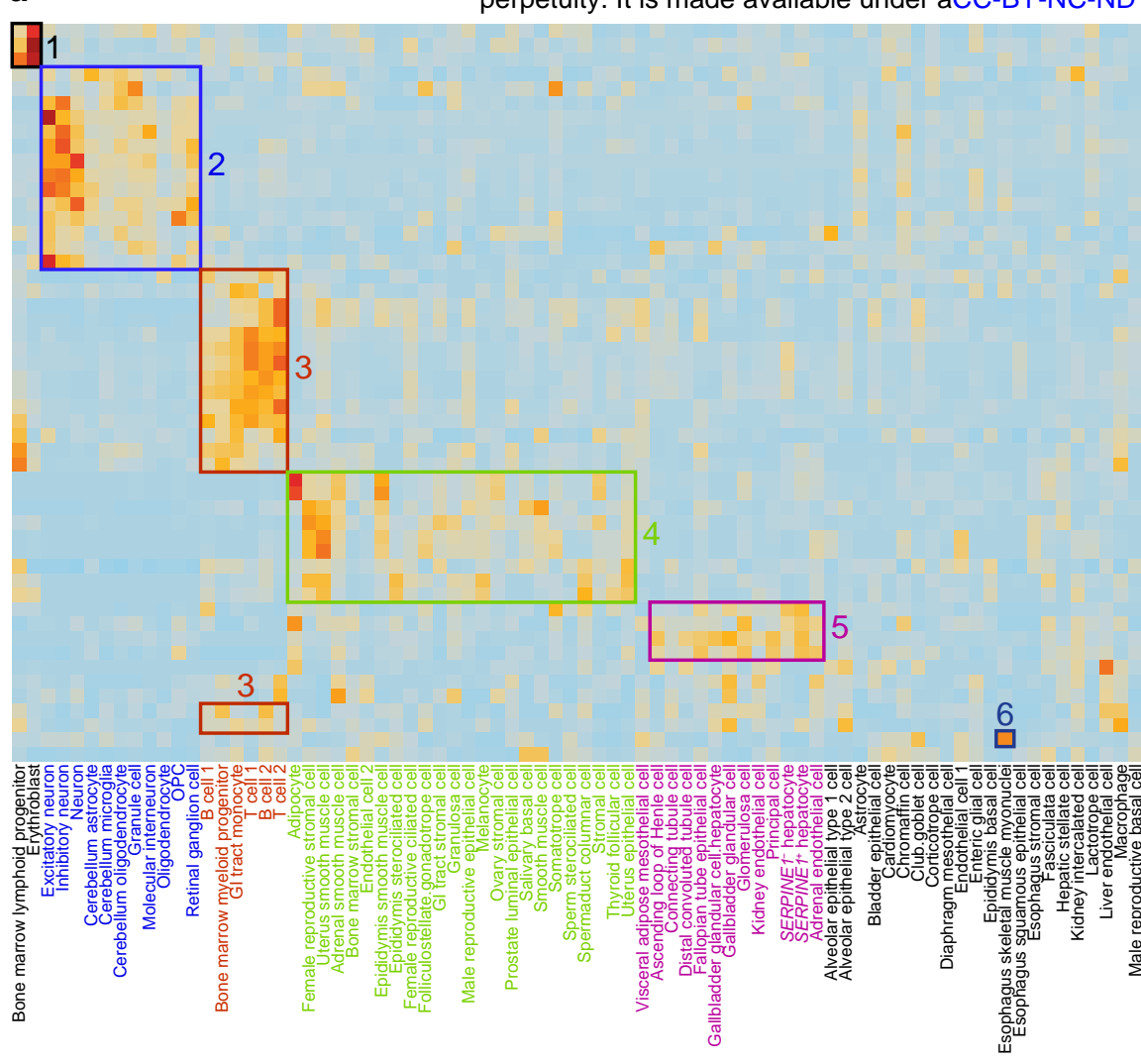

b
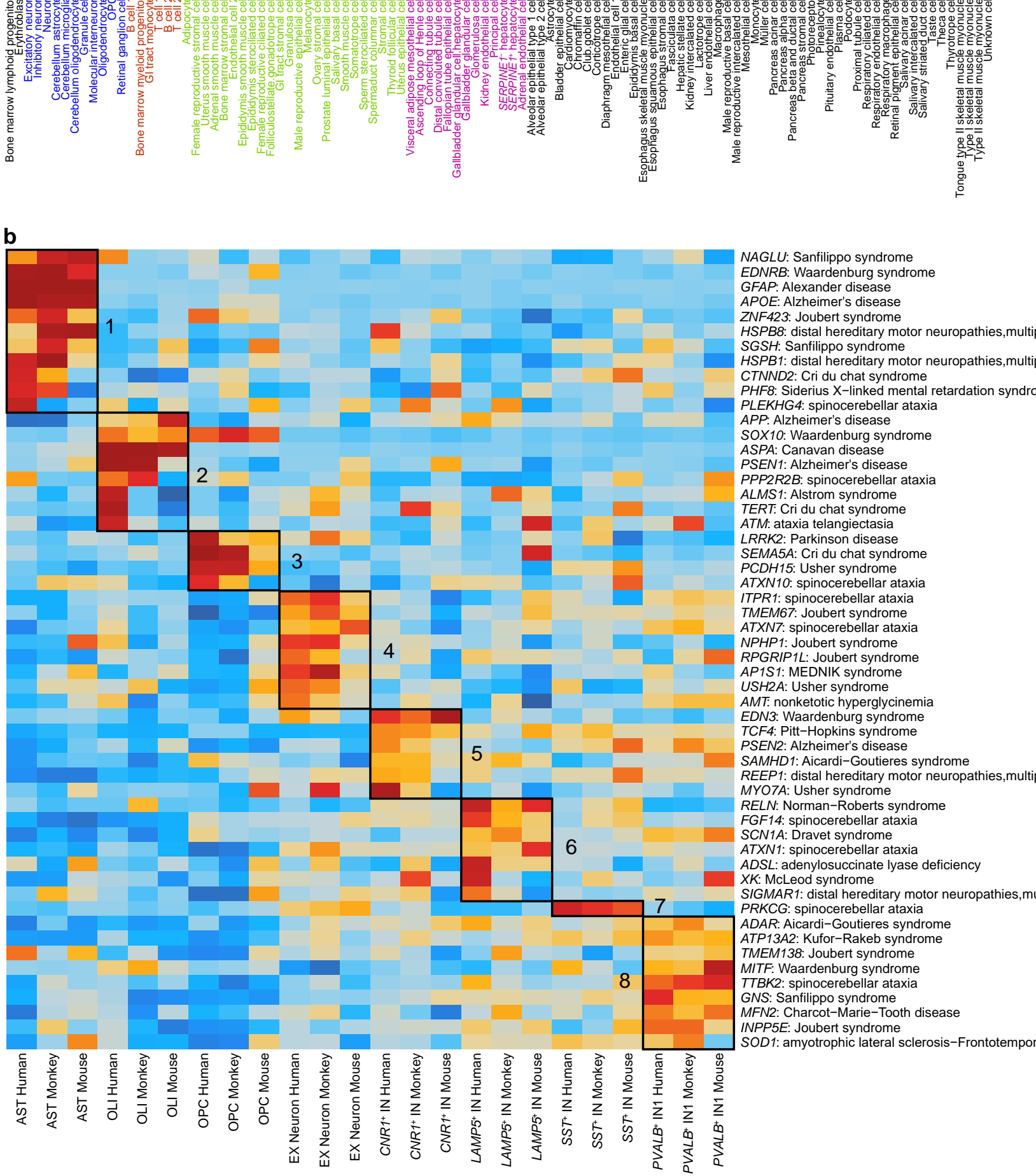

\section{Z-score} Low High Sphered Cell Volu
sal Metabolic Rate Duroticism Pepression Years of Education
College Education norninia Malign
ALS Anheumatoid arthritis
Crohn's disease Respiratory and Ear-nose-throat Diseases Allergy eczema diagnosed
Autolmmune Traits Thyroid any self rep Dypothyroildism
Osinophil Count

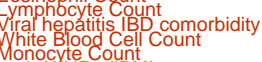
Aaist-nipRatio orced Vital Capacity FVC
ung FVCZSMOKE T2D Dh direct 1 rep Malignant neoplasm of brain 6 AD Kansenet
A Kunkle
ADHD 
bioRxiv preprint doi: https://doi.org/10.1101/2021.12.13.472311; this version posted December 13, 2021. The copyright holder for this

preprint (which was not certified by peer review) is the author/funder, who has granted bioRxiv a license to display the preprint in

ㅇ F1 (11,191 nuclei) ㅇ F3 (13,126 nuclei)

o F3 (40,932 nuclei) 今 M1 (58,139 nuclei)

ㅇ F1 (7,483 nuclei) 今 M2 (12,090 nuclei)

우 F1 (3,300 nuclei)

o F3 (18,479 nuclei)

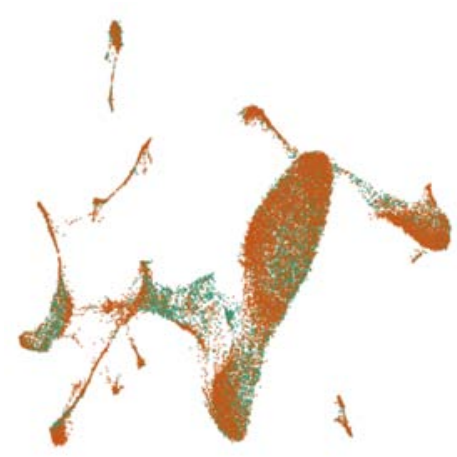

Cerebellum

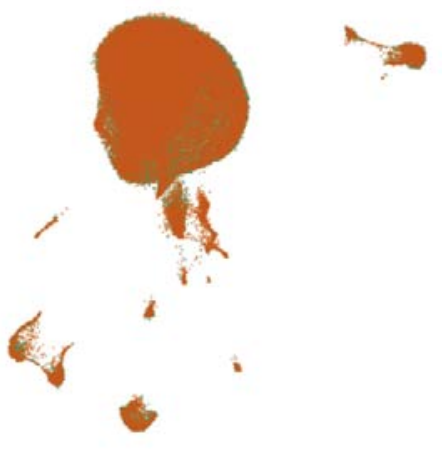

\section{Diaphragm}

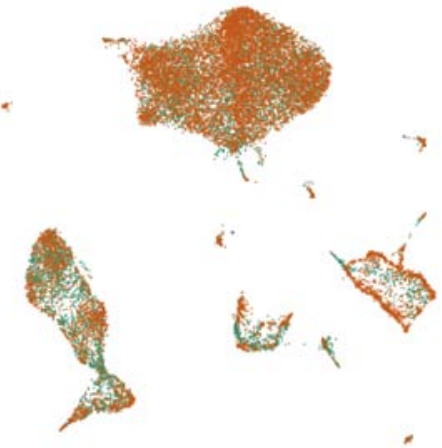

Gallbladder

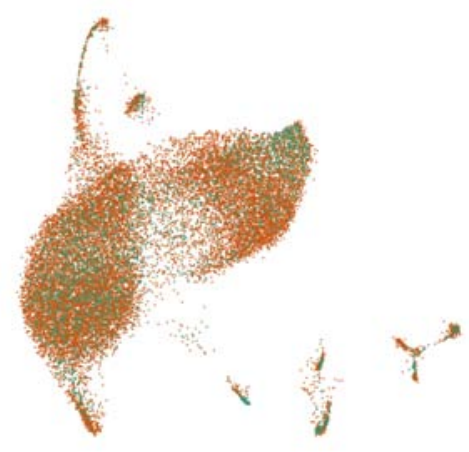

Kidney

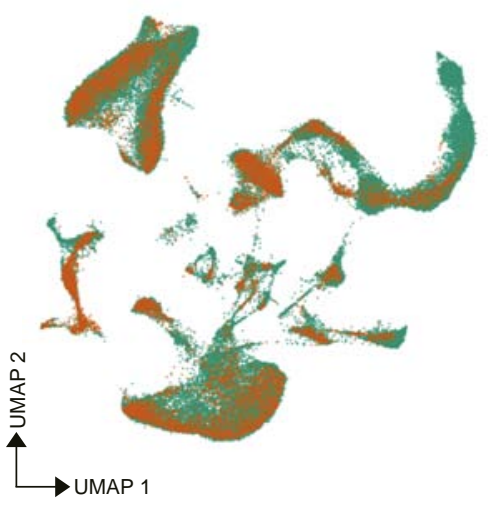

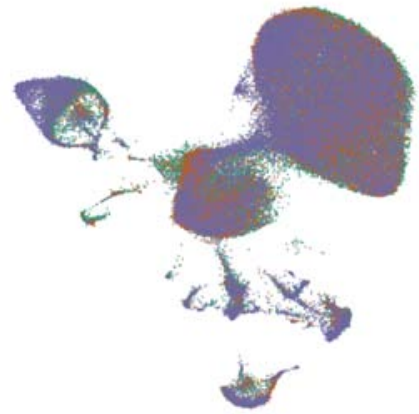

Lung

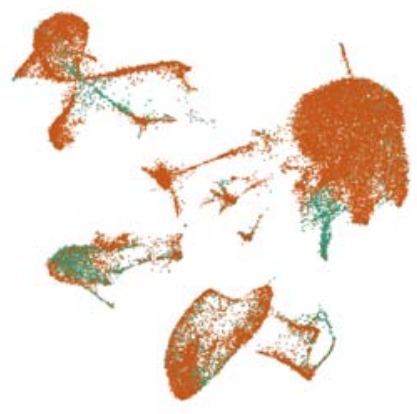

Salivary gland

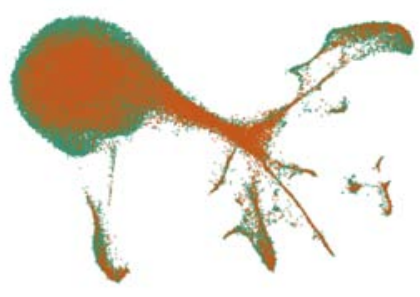

3

Subcutaneous adipose
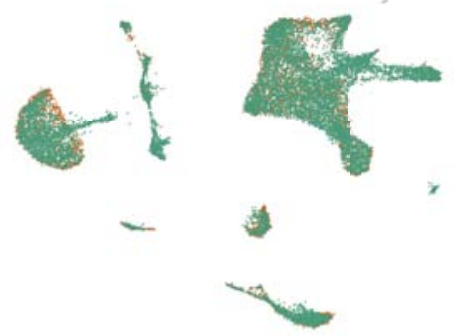

F1 (13,453 nuclei)

\& M2 (4,728 nuclei)

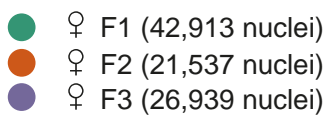

\& F1 (8,059 nuclei)

ㅇ F3 (25,995 nuclei)
- F3 (18,393 nuclei)

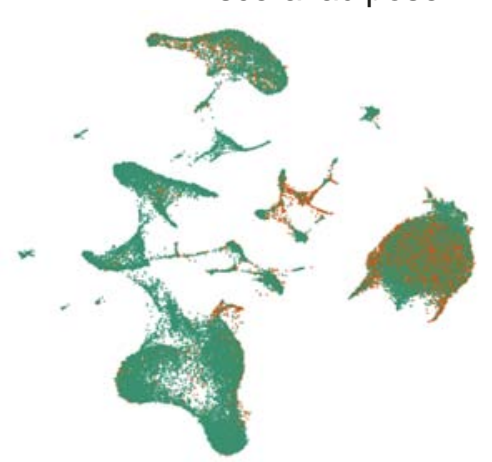

- $q$ F1 (23,658 nuclei)

今 M2 (20,320 nuclei) 
bioRxiv preprint doi: https://doi.org/10.1101/2021.12.13.472311; this version posted December 13,2021 . The copyright holder for this Cardiovascular which was not certified by peer review) is the author/funder, who has granted bioRxiv a license to display the preprint in

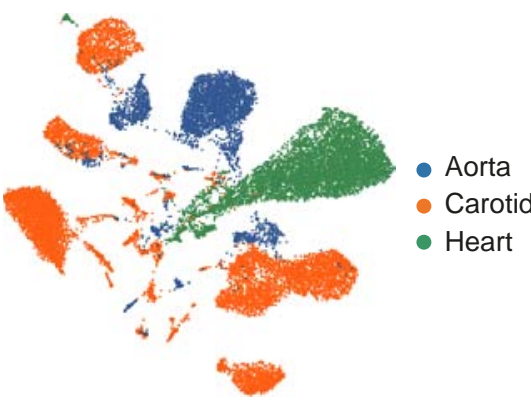

Skeletal

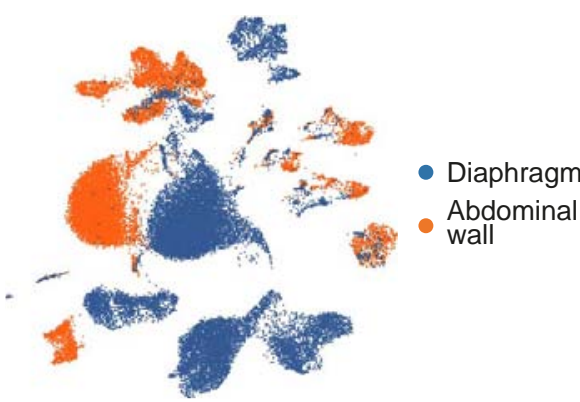

Respiratory

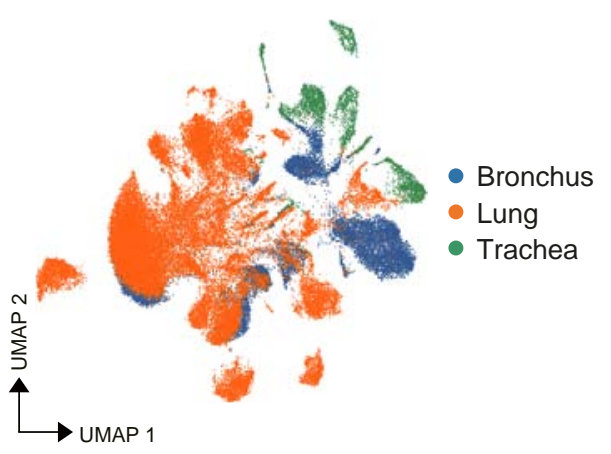

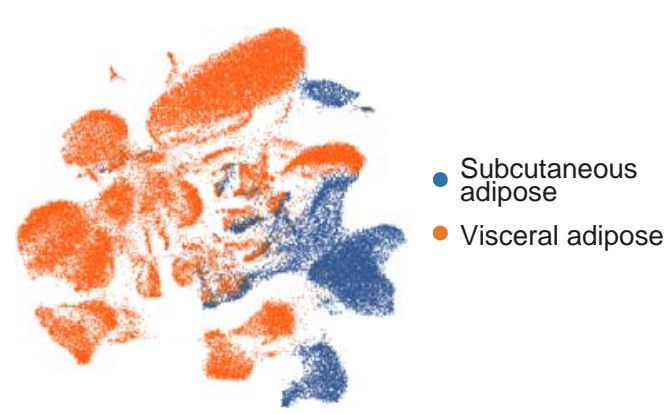

Central nervous

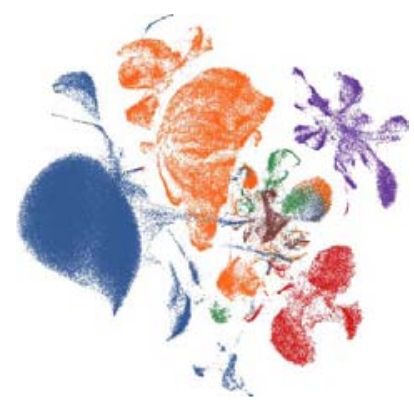

Cerebellum

- Neocortex

- Substantia nigra

- Pigmentary epi

- Retina

- Spinal cord

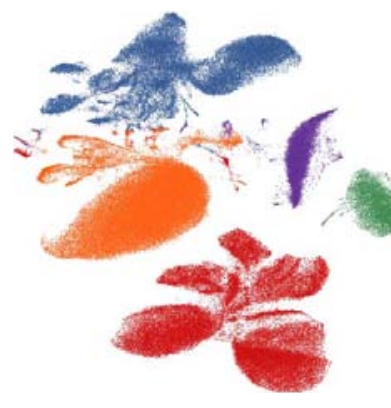

- Adrenal gland

- Pancreas

- Pineal gland

- Pituitary gland

- Thyroid
Reproductive (male)

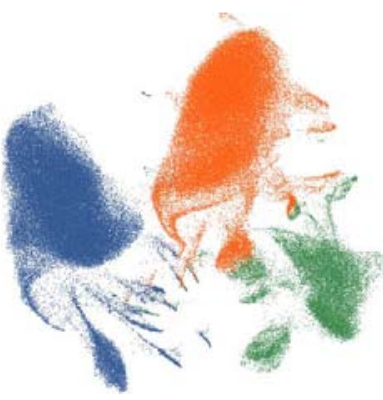

- Epididymis

- Prostate

- Spermaduct 
bioRxiv preprint doi: https://doi.org/10.1101/2021.12.13.472311; this version posted December 13, 2021. The copyright holder for this

preprint (which was not certified by peer review) is the author/funder, who has granted bioRxiv a license to display the preprint in Abdominal wall $(12,117$ perpetuity. It is made available under aCC-BY-NC-ND 4.0 International license.

Aorta (3,960 nuclei)
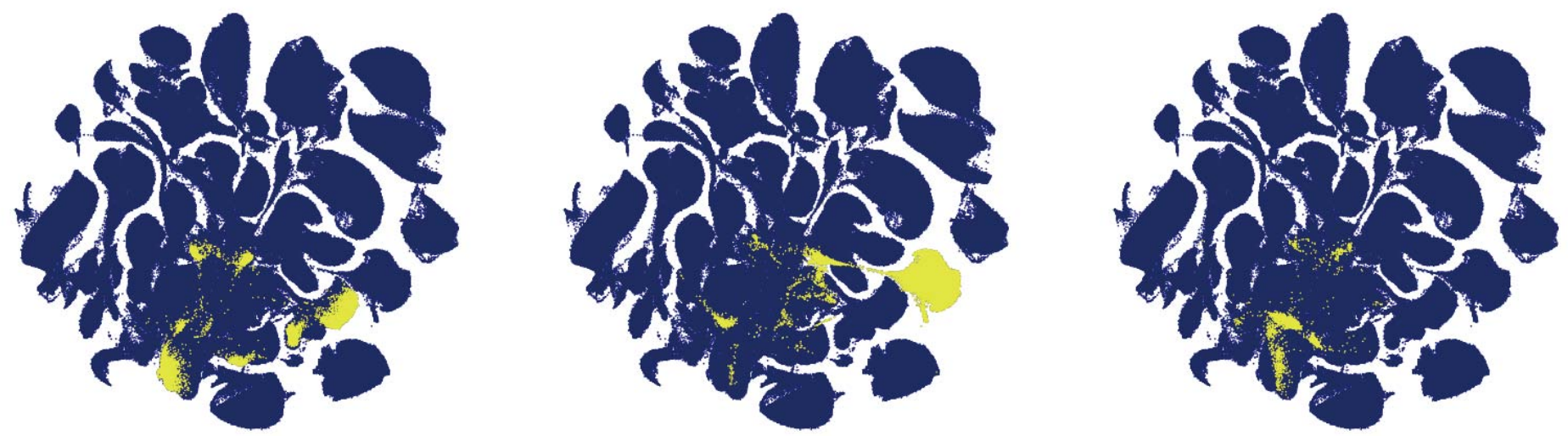

Bladder (24,317 nuclei)

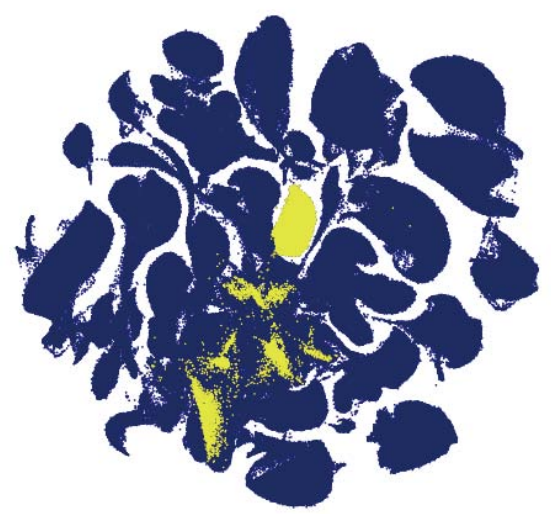

Bone marrow $(7,880$ cells $)$

Bronchus (11,225 nuclei)
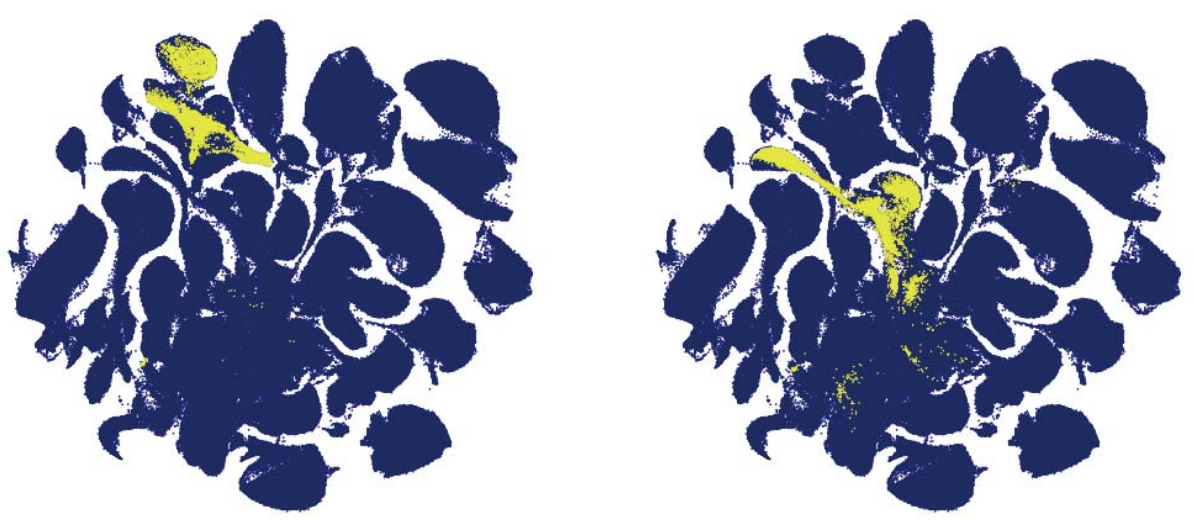

Carotid (10,071 nuclei)

Cerebellum (99,071 nuclei)
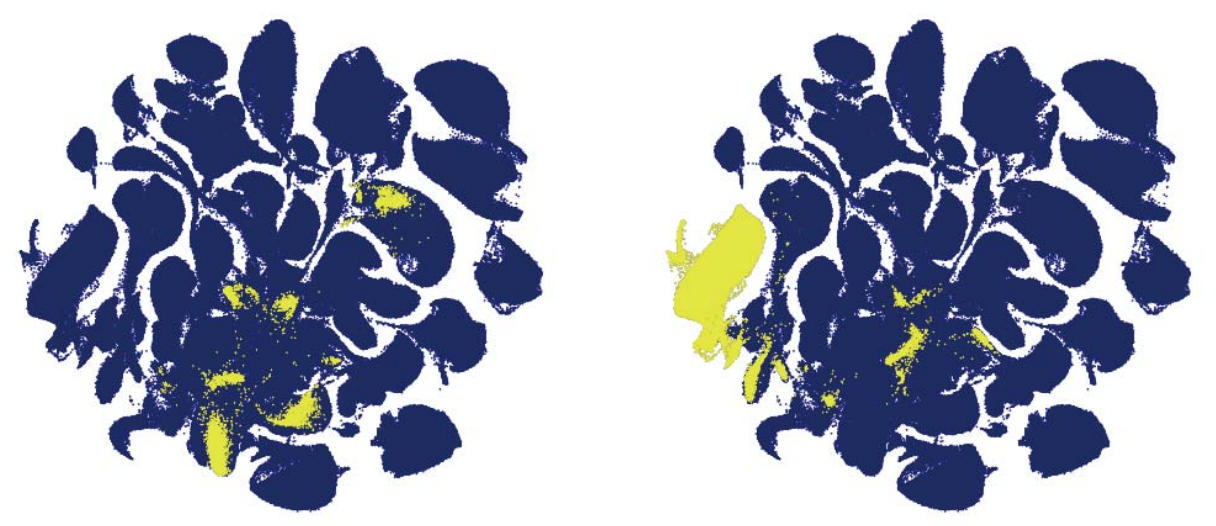

Colon (6,554 cells)

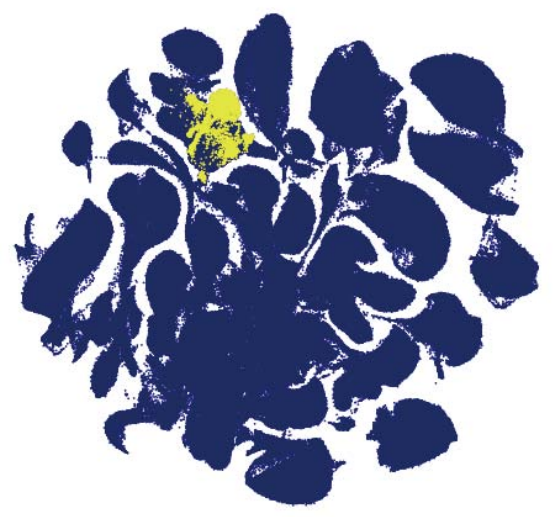

Duodenum (2,039 cells)

Epididymis (59,647 nuclei)
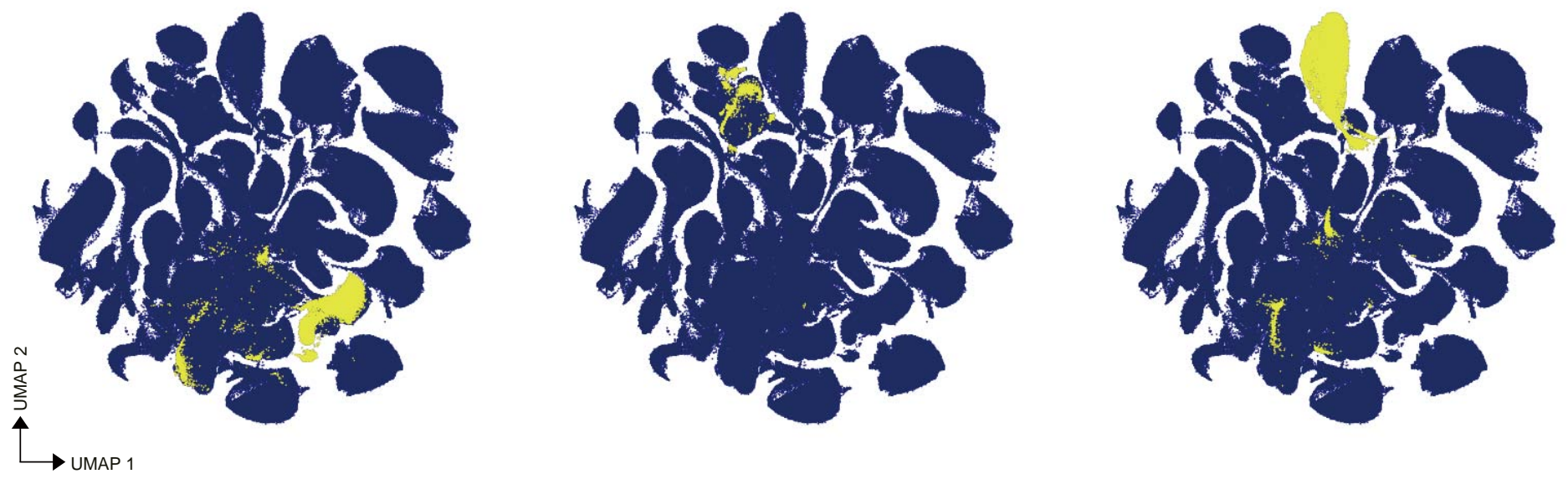
bioRxiv preprint doi: https://doi.org/10.1101/2021.12.13.472311; this version posted December 13,2021 . The copyright holder for this

preprint (which was not certified by peer review) is the author/funder, who has granted bioRxiv a license to display the preprint in

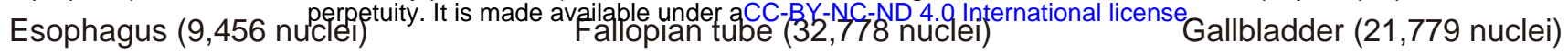

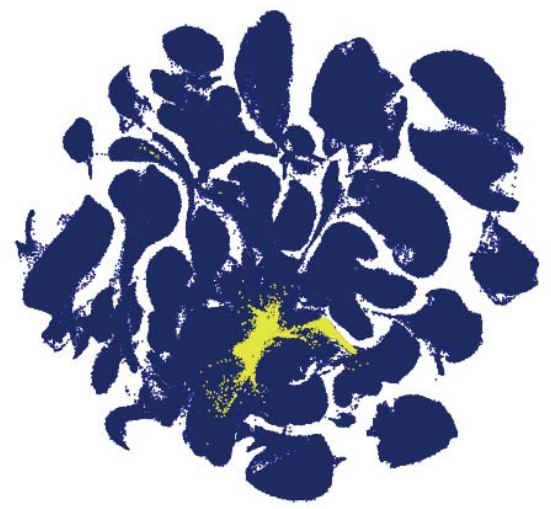

Heart $(5,881$ nuclei)

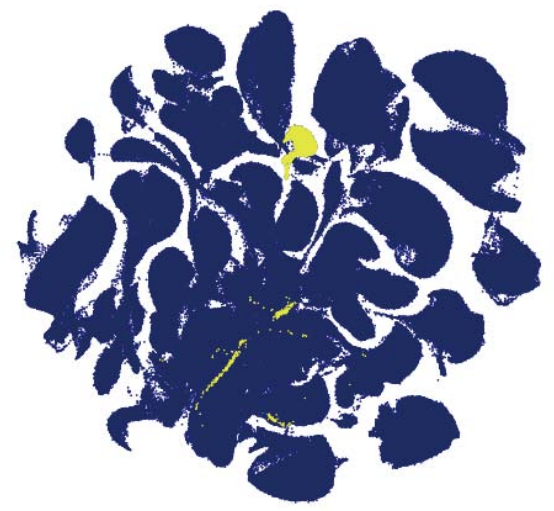

Lung (34,054 nuclei)

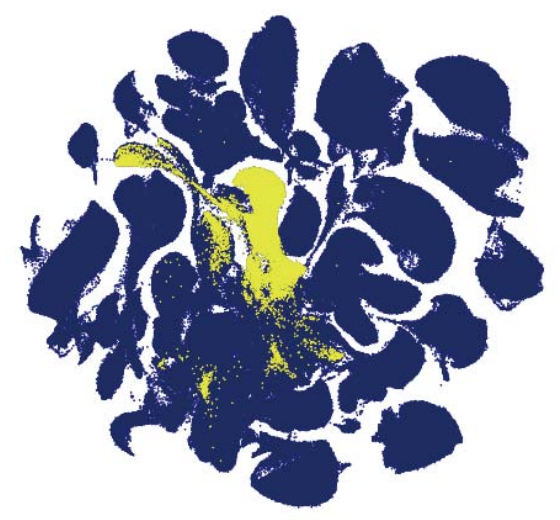

Ovary $(35,764$ nuclei)

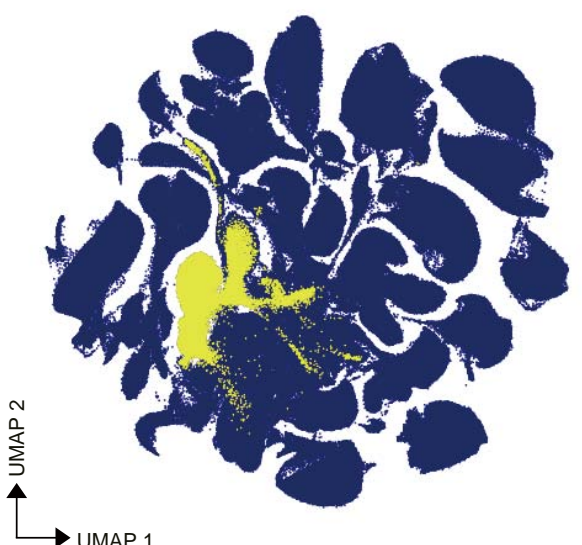

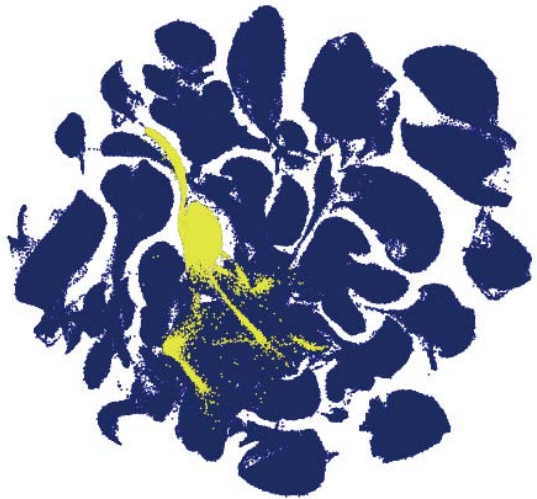

Kidney $(62,873$ nuclei)

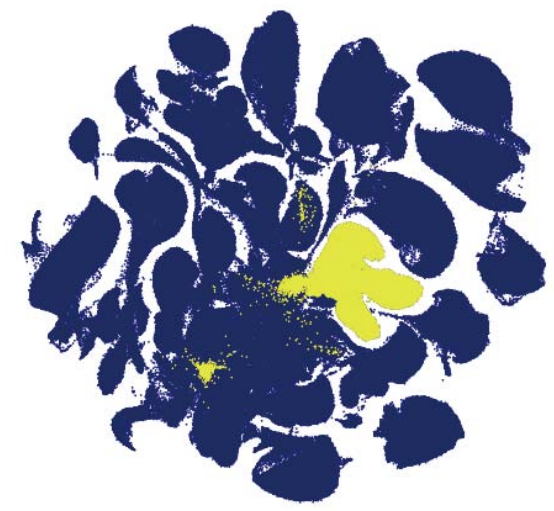

Lymph node (8,413 cells)

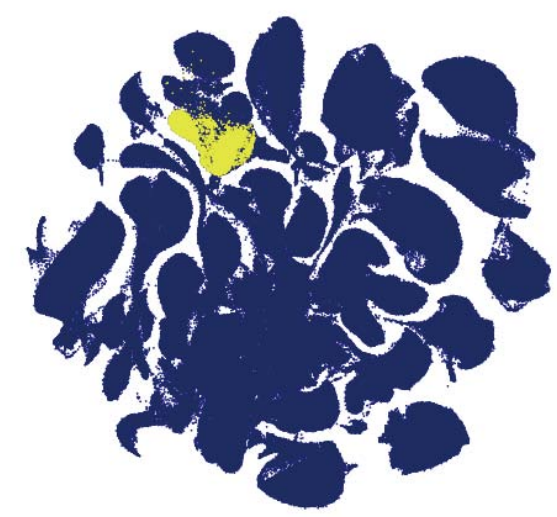

Pancreas (51,480 nuclei)

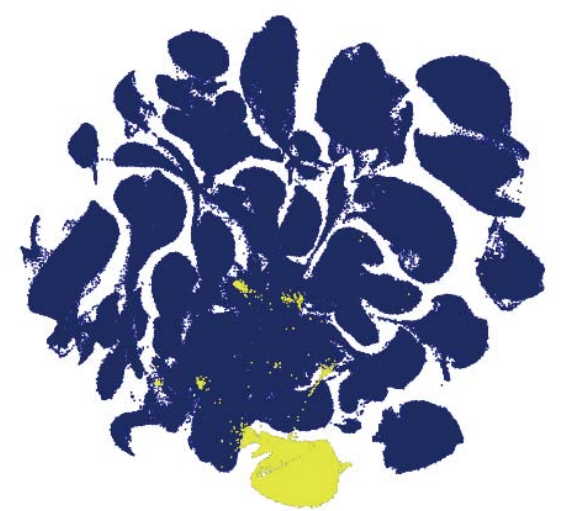

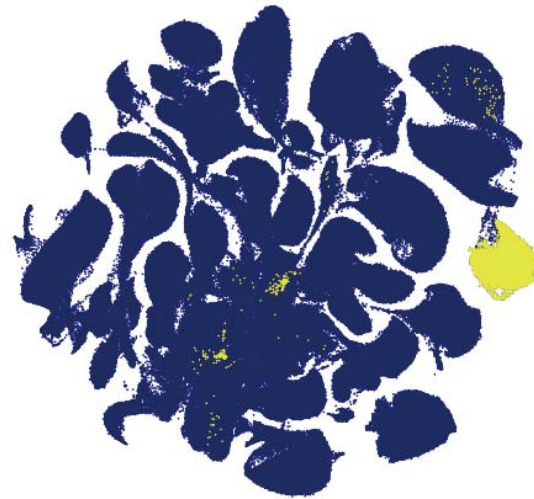

Liver (91,389 nuclei)

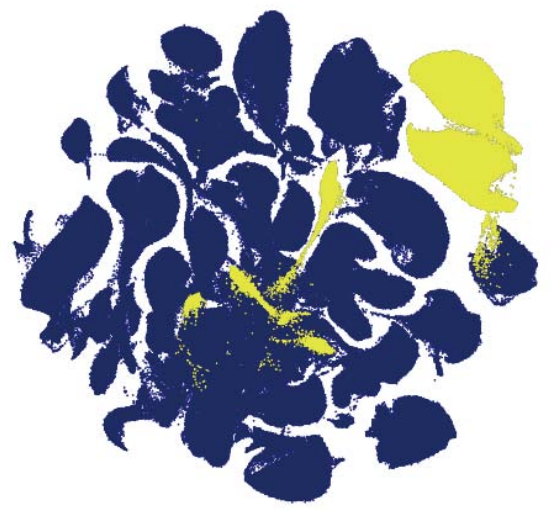

Neocortex (38,367 nuclei)

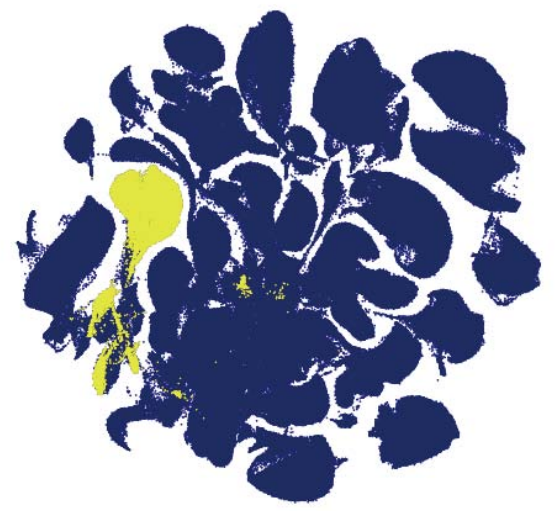

PBMC (15,728 cells)

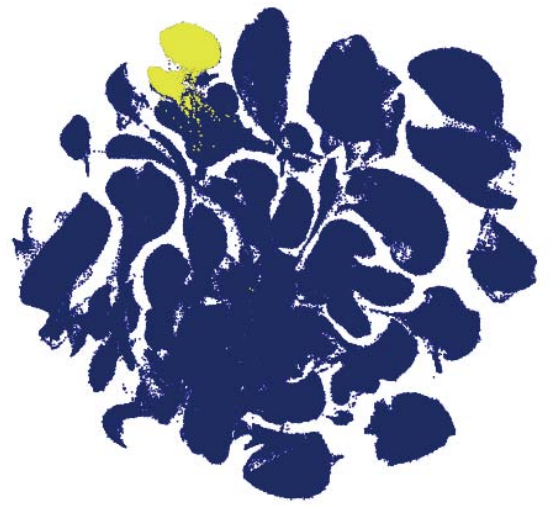


bioRxiv preprint doi: https://doi.org/10.1101/2021.12.13.472311; this version posted December 13, 2021. The copyright holder for this

preprint (which was not certified by peer review) is the author/funder, who has granted bioRxiv a license to display the preprint in

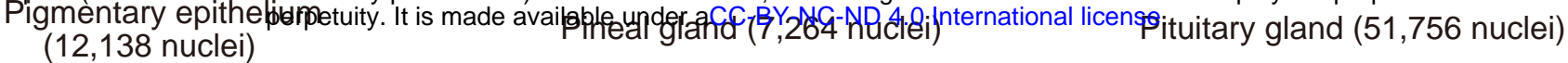

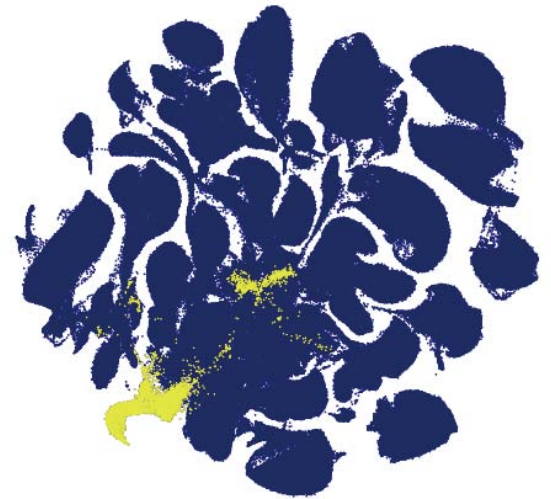

Prostate $(51,928$ nuclei)

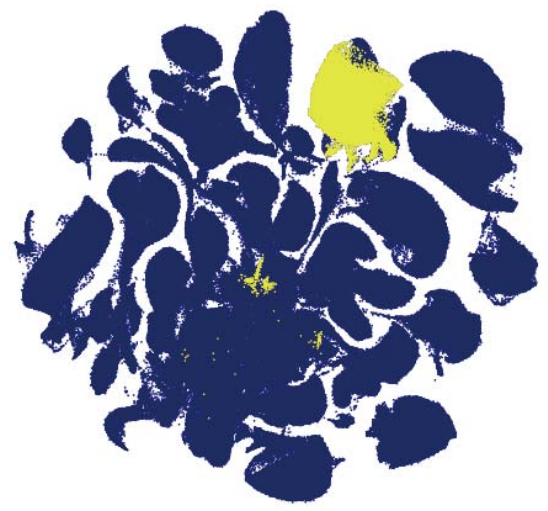

Spermaduct $(18,973$ nuclei)

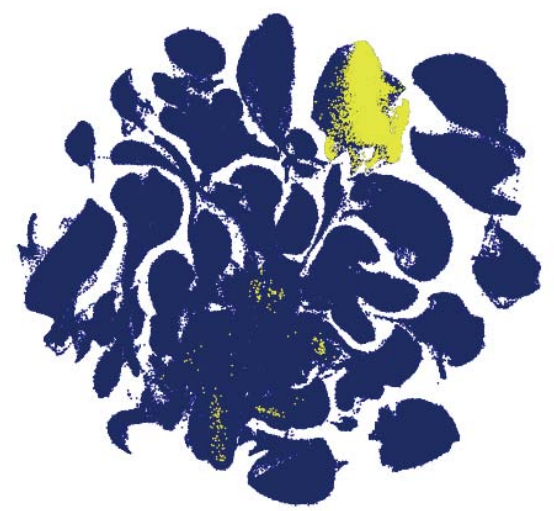

Stomach $(3,419$ cells)

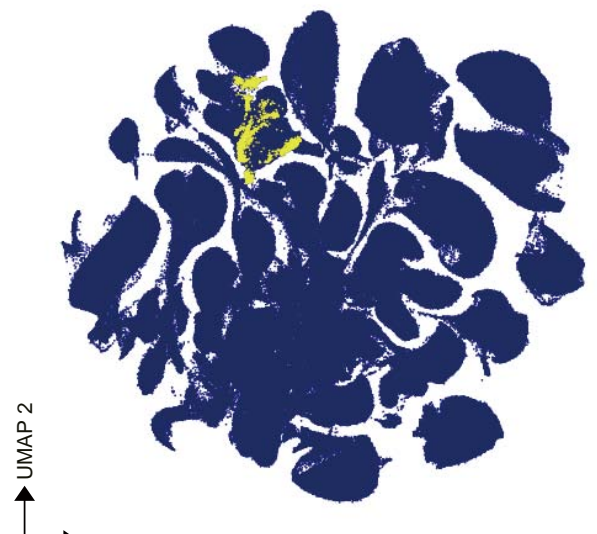

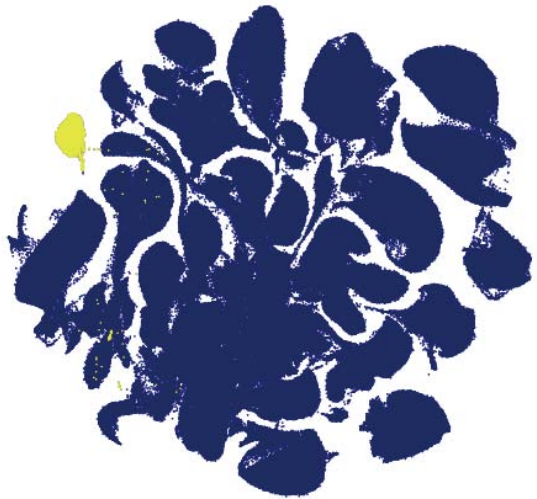

Retina $(12,003$ nuclei)

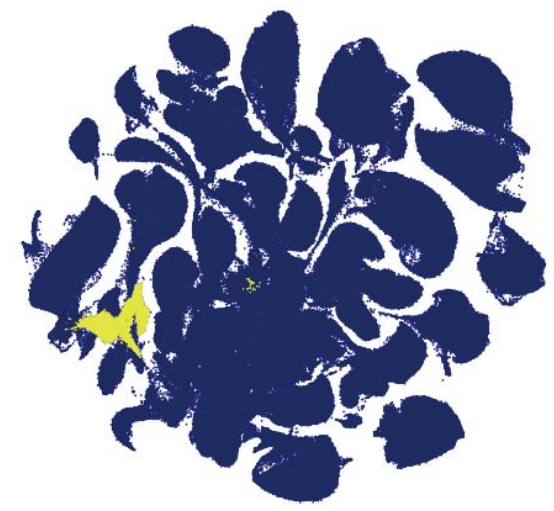

Spinal cord (3,952 nuclei)

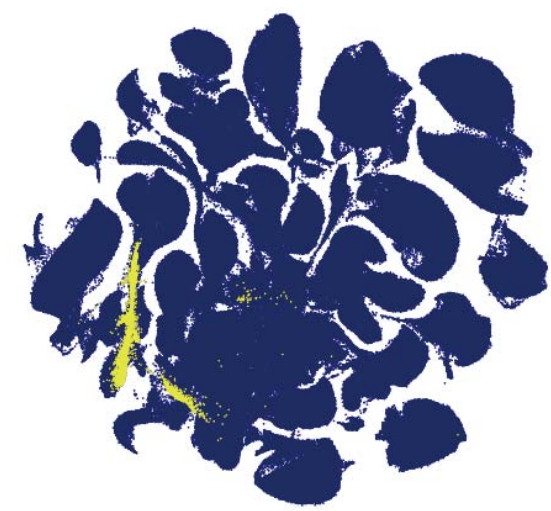

Subcutaneous adipose $(18,181$ nuclei)

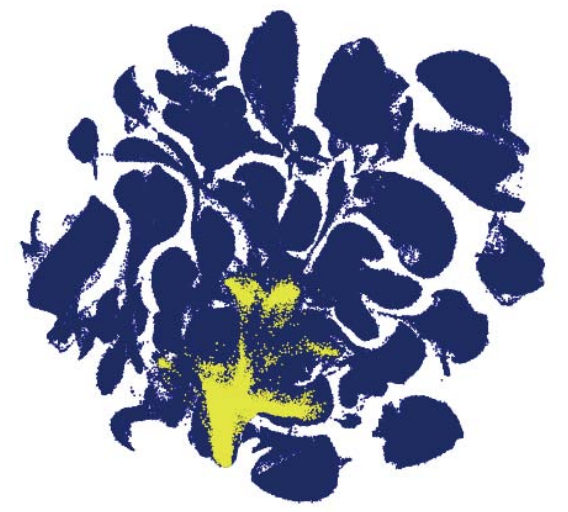

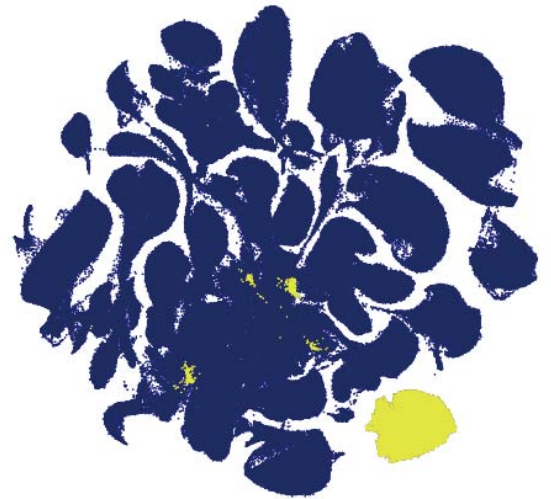

Salivary gland (74,461 nuclei)

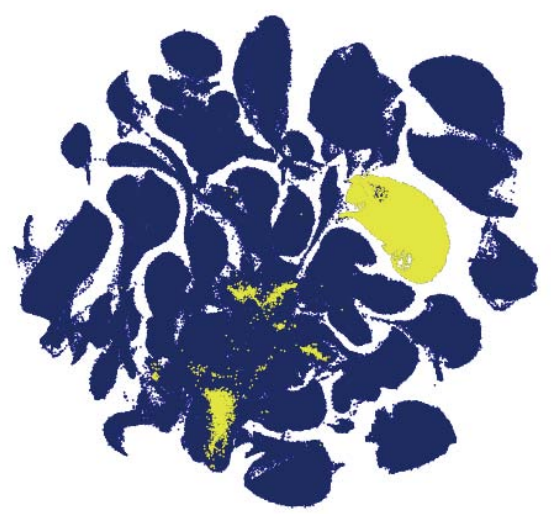

Spleen $(6,464$ cells)

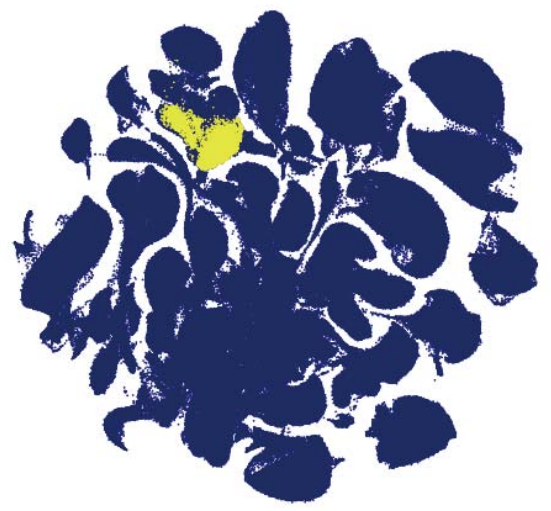

Substantia nigra (4,271 nuclei)

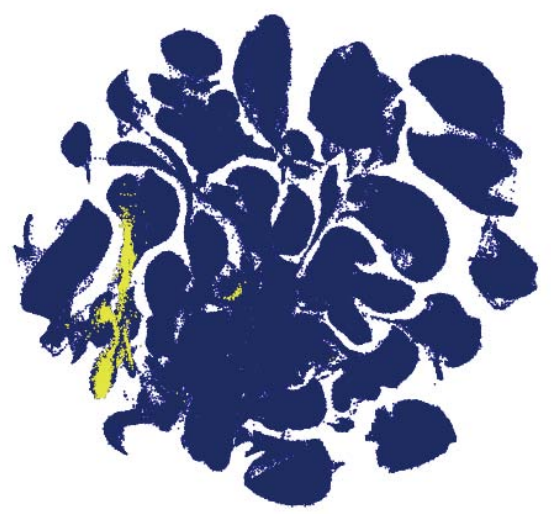


bioRxiv preprint doi: https://doi.org/10.1101/2021.12.13.472311; this version posted December 13, 2021. The copyright holder for this

preprint (which was not certified by peer review) is the author/funder, who has granted bioRxiv a license to display the preprint in

Thyroid (11,643 nuclei) petuity. It is made available under aCG-BY Y-NC-ND 40 O International license.

Tonsil (8,150 nuclei)

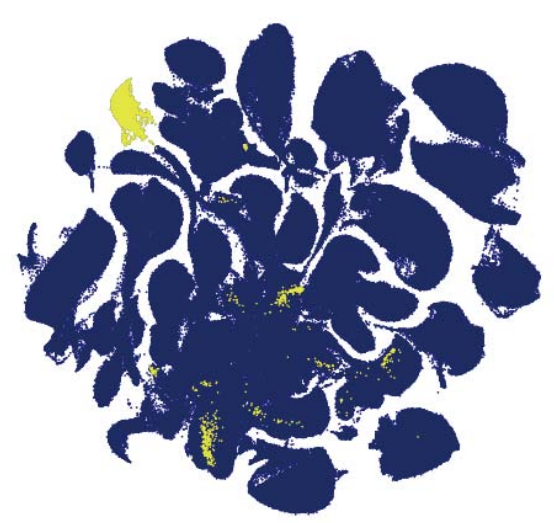

Trachea (3,705 nuclei)

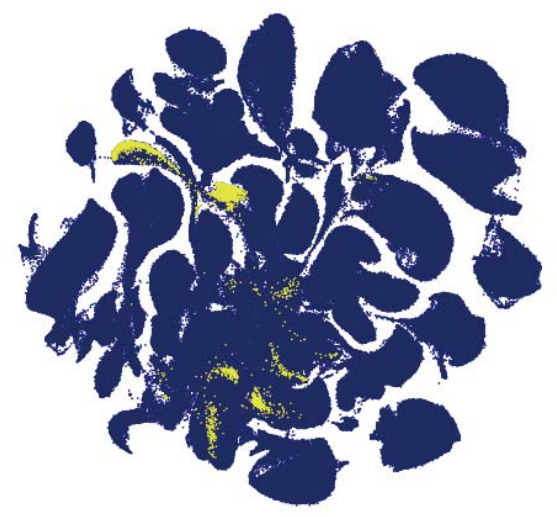

Visceral adipose (43,978 nuclei)

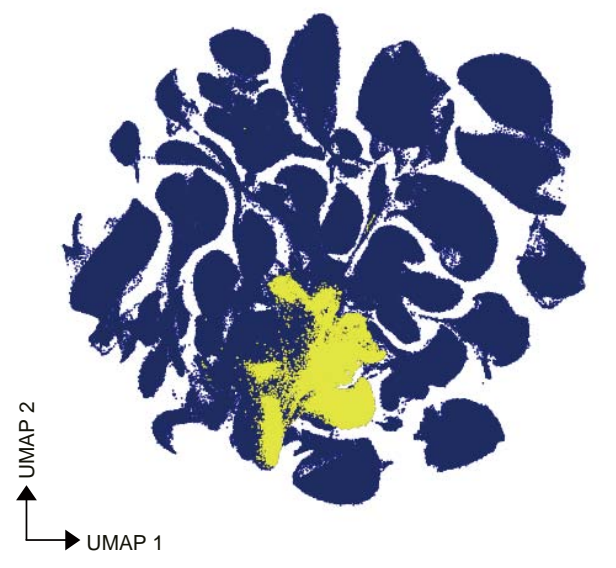

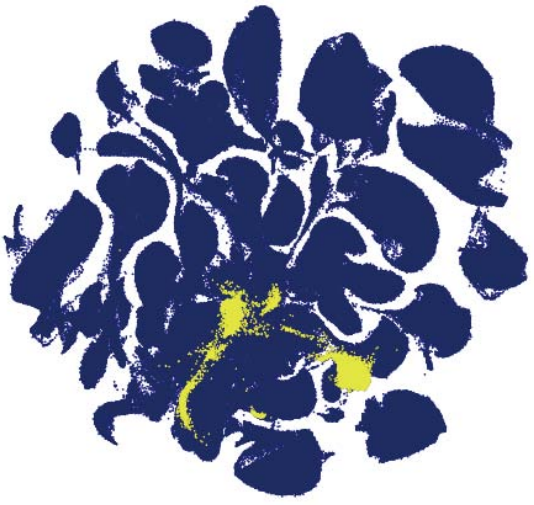

Uterus $(35,964$ nuclei)

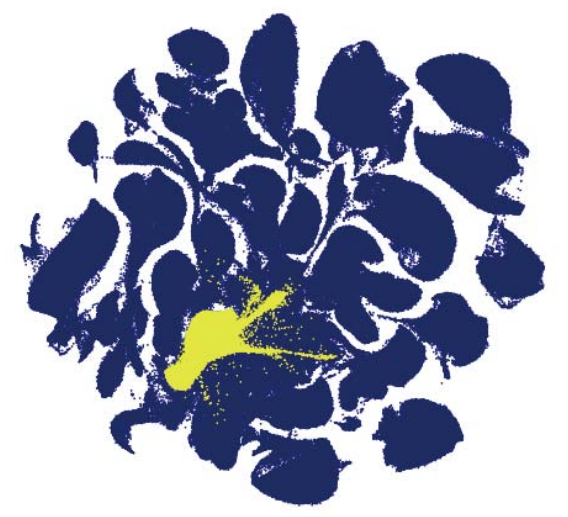

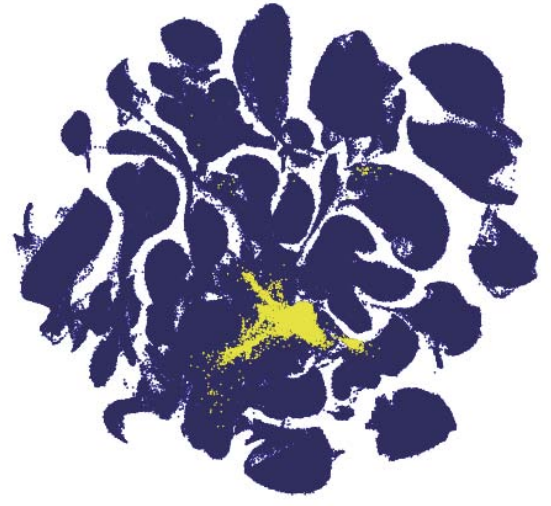

Vagina (2,265 nuclei)

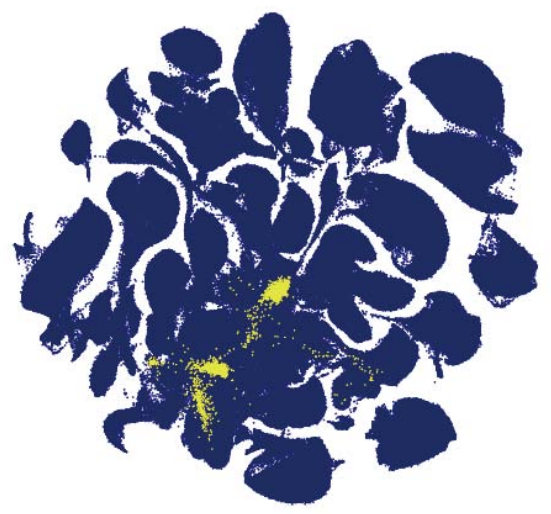


bioRxiv preprint doi: https://doi.org/10.1101/2021.12.13.472311; this version posted December 13,2021 . The copyright holder for this

preprint (which was not certified by peer review) is the author/funder, who has granted bioRxiv a license to display the preprint in

Abdominal wall (12,117 nuclei)
nupetulty. It made available under aCC-BY-NC-ND 4.0 International license.
Adrenal gland (35,296 nuclei)

Aorta (3,960 nuclei)

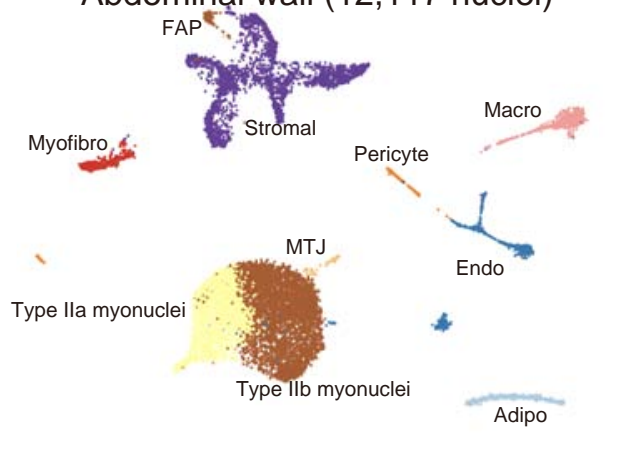

Satellite

Type I myonuclei

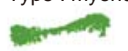

Bladder (24,317 nuclei)

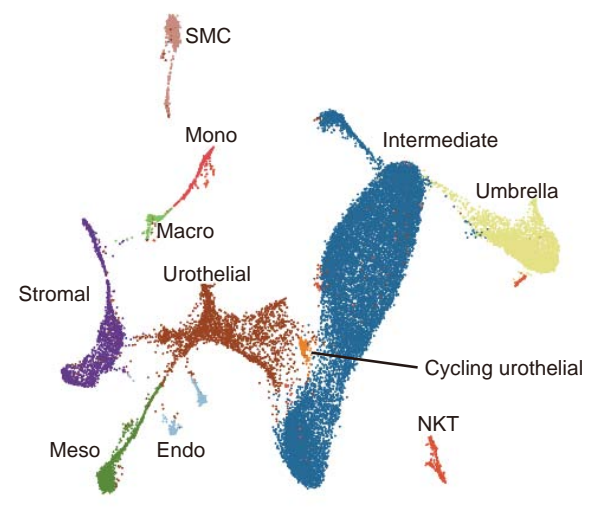

Carotid (10,071 nuclei)

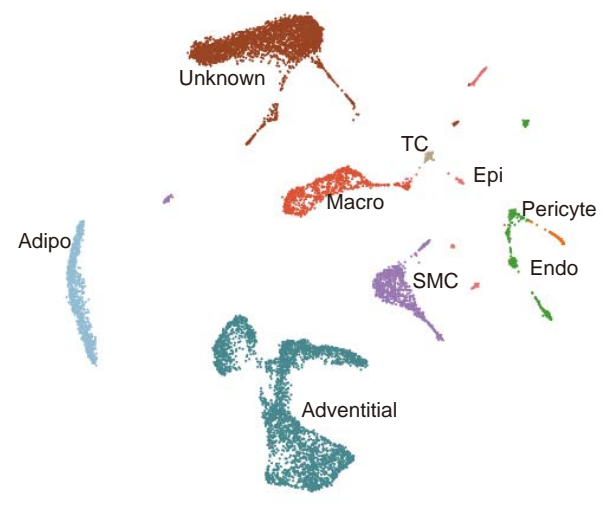

Diaphragm (19,573 nuclei)

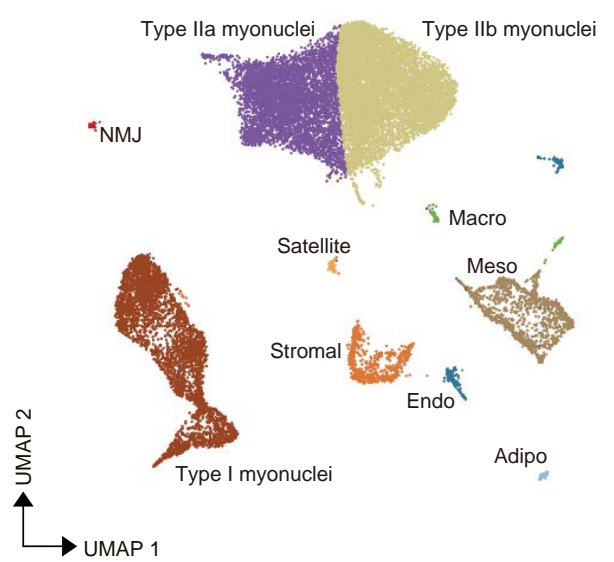

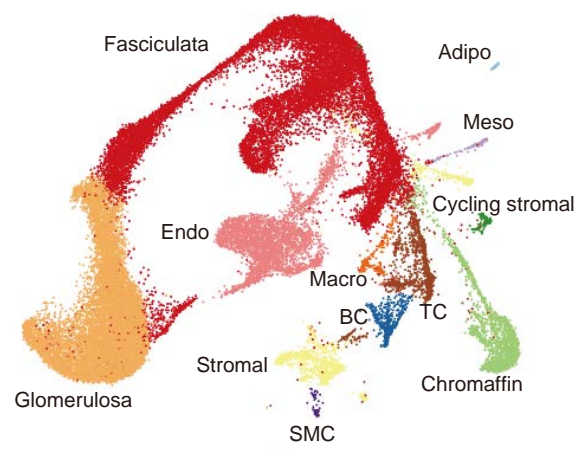

Bone marrow ( 7,880 cells)

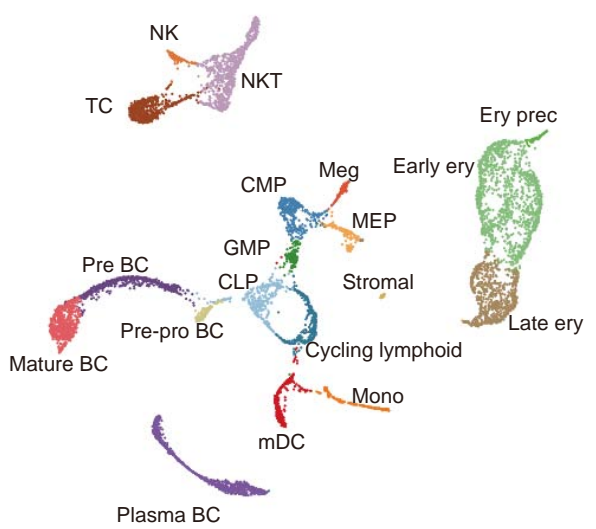

Cerebellum $(99,071$ nuclei)
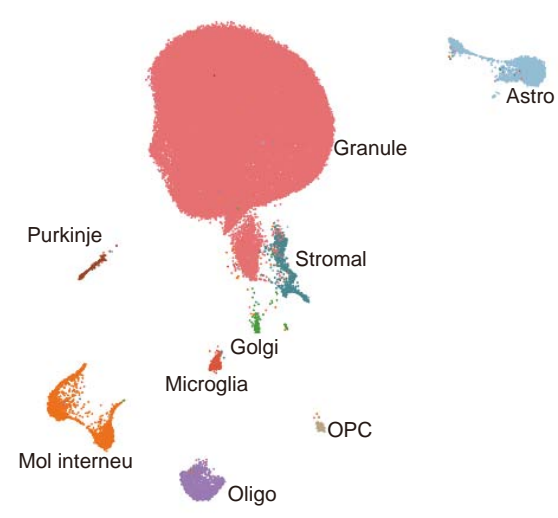

Duodenum (2,039 cells)

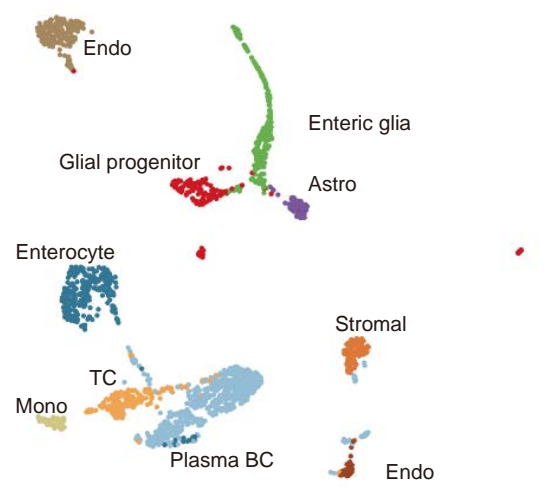

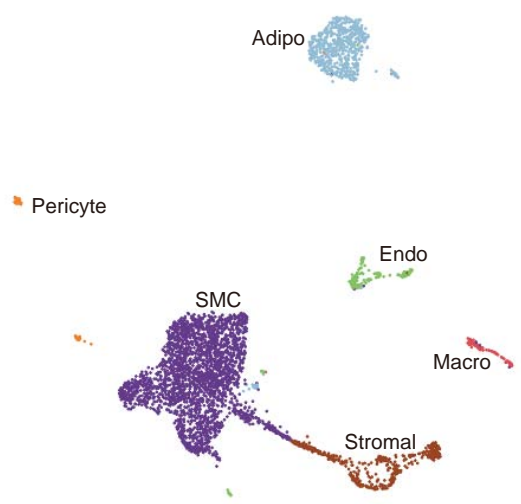

Bronchus (11,225 nuclei) x Neuroendocrine
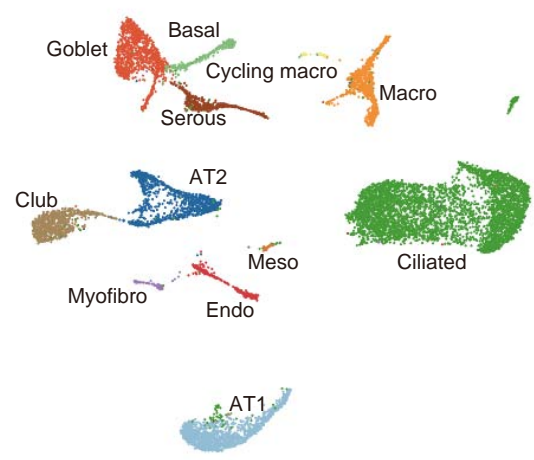

Colon (6,554 cells)

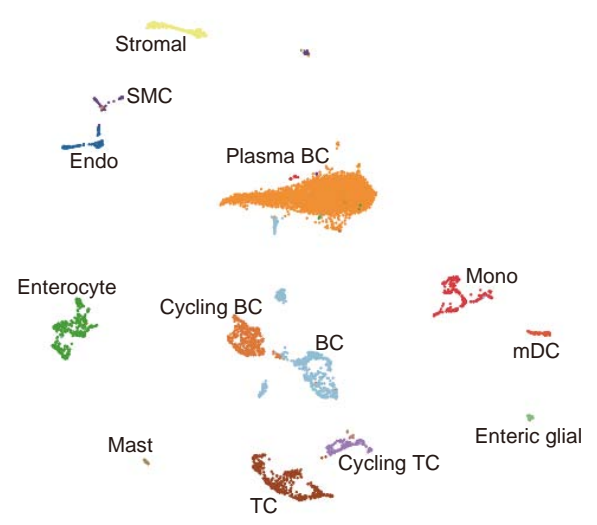

Epididymis (59,647 nuclei)

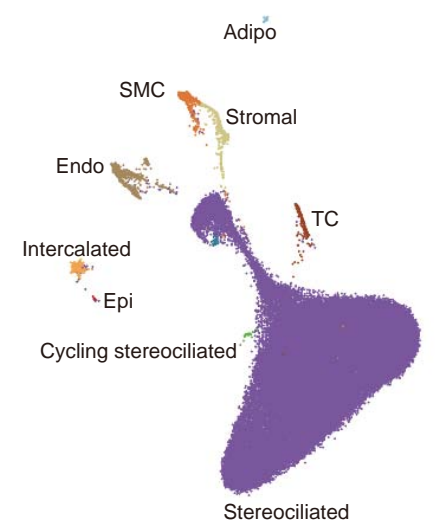


bioRxiv preprint doi: https://doi.org/10.1101/2021.12.13.472311; this version posted December 13,2021 . The copyright holder for this preprint (which was not certified by peer review) is the author/funder, who has granted bioRxiv a license to display the preprint in

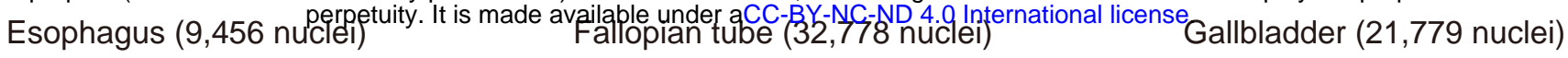

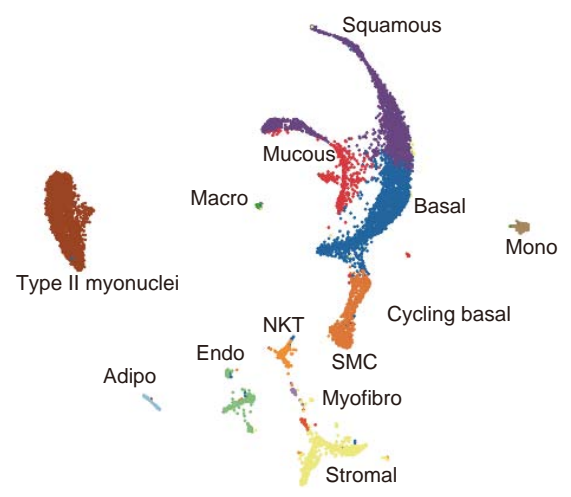

Heart (5,881 nuclei)

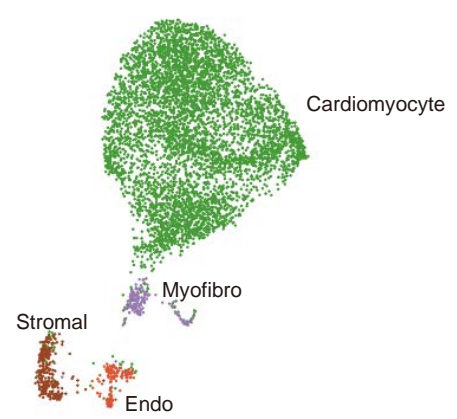

Adipo

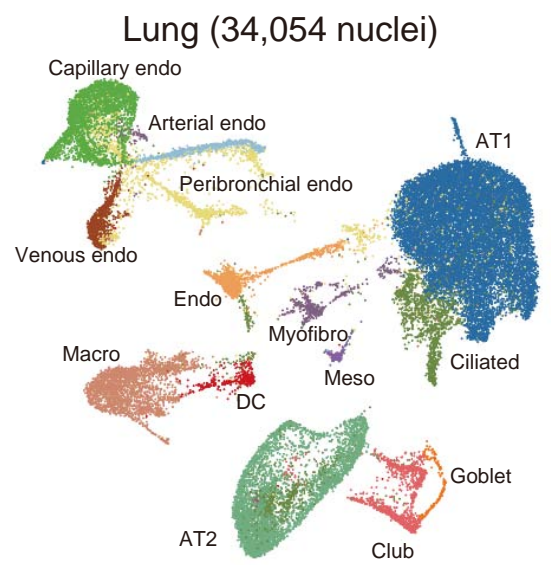

Ovary $(35,764$ nuclei)

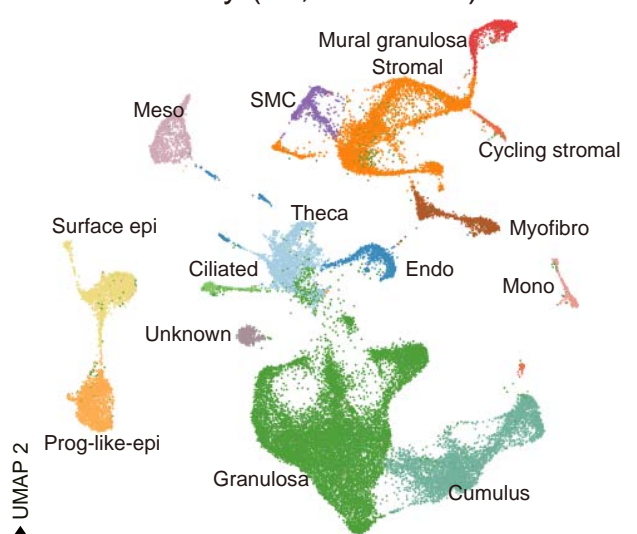

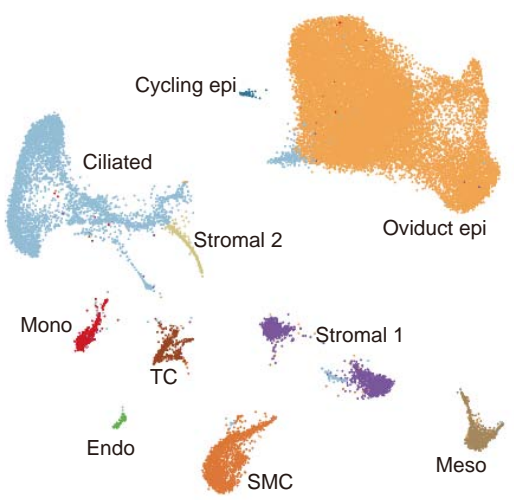

Kidney $(62,873$ nuclei)

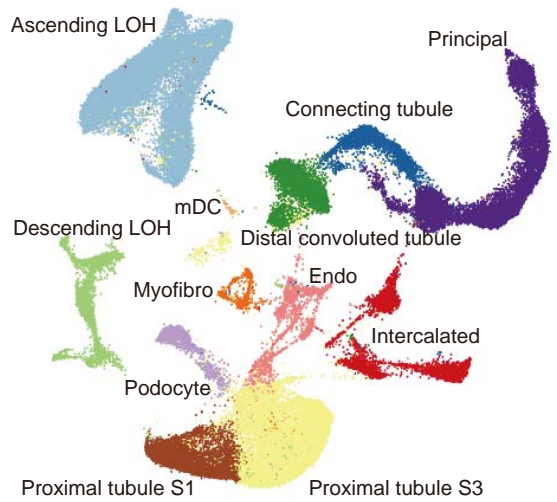

Lymph node (8,413 cells)

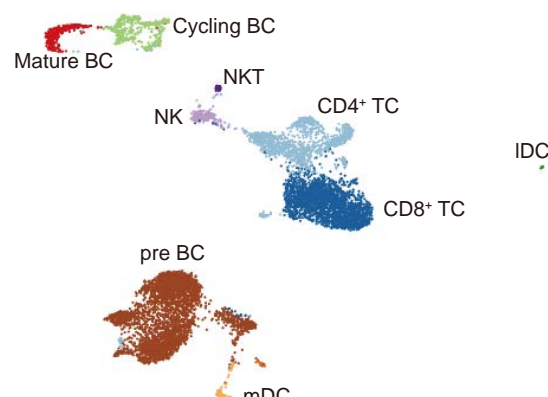

Plasma BC

Mast .

Mono

Pancreas (51,480 nuclei)

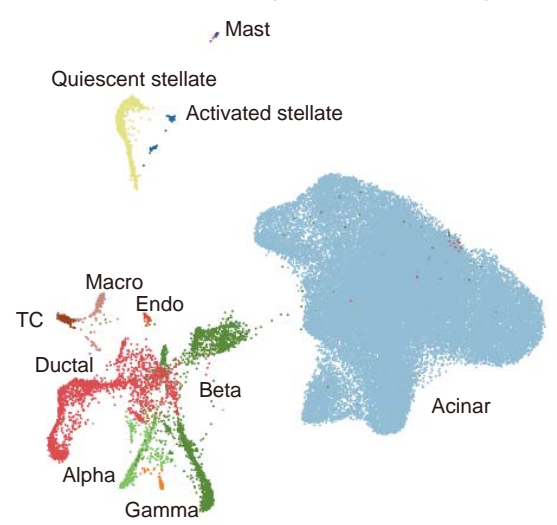

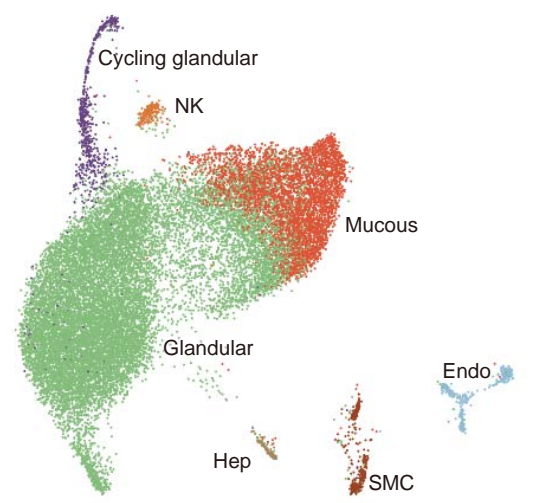

Liver (91,389 nuclei)

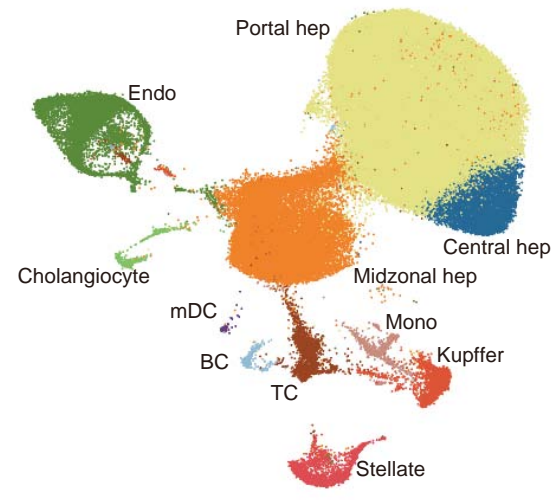

Neocortex (38,367 nuclei)

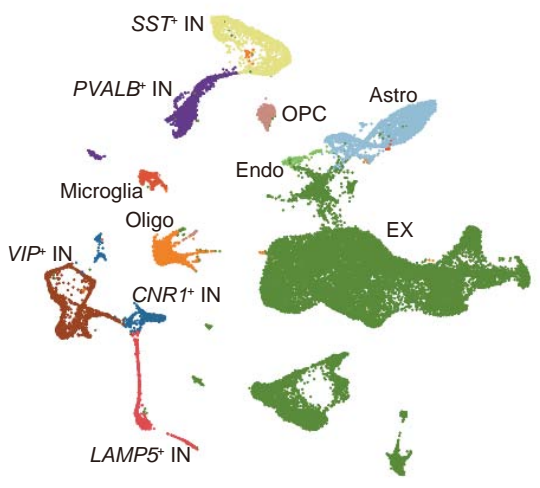

PBMC (15,728 cells)
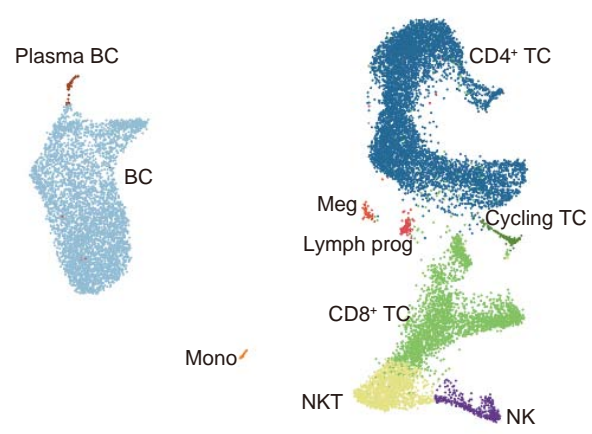

Neutro $=$ 
bioRxiv preprint doi: https://doi.org/10.1101/2021.12.13.472311; this version posted December 13, 2021. The copyright holder for this

preprint (which was not certified by peer review) is the author/funder, who has granted bioRxiv a license to display the preprint in

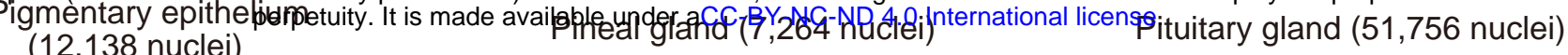
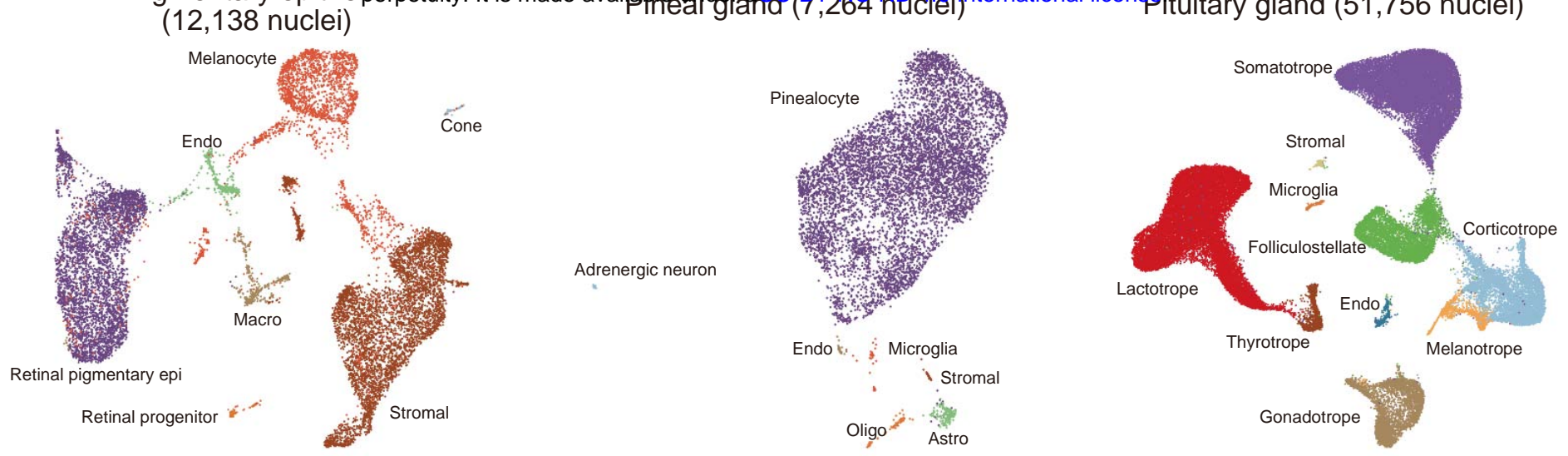

Prostate (51,928 nuclei)

Retina $(12,003$ nuclei)

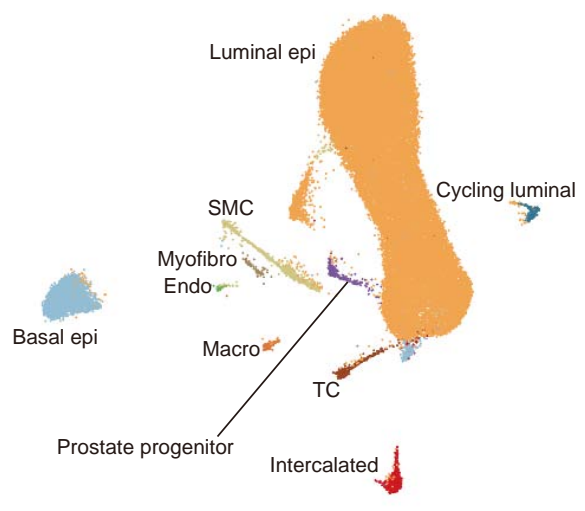

Spermaduct $(18,973$ nuclei)

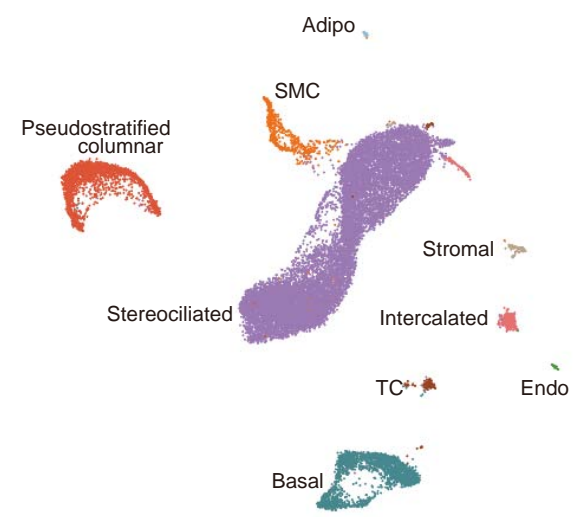

Stomach $(3,419$ cells $)$
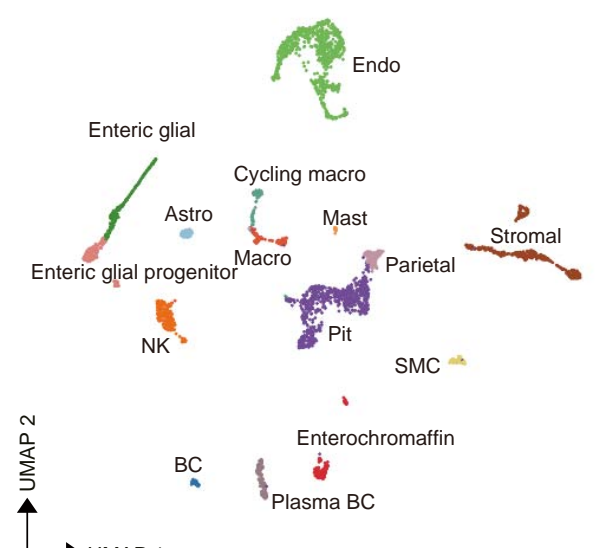

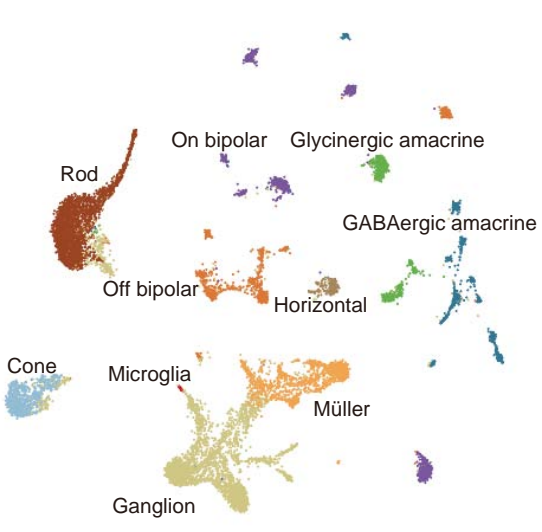

Spinal cord (3,952 nuclei)

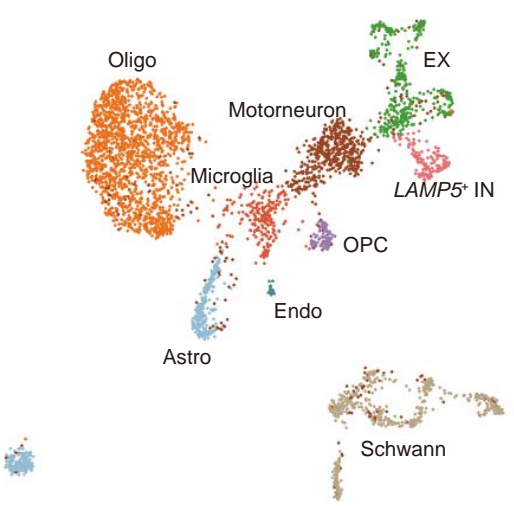

Subcutaneous adipose

$(18,181$ nuclei)

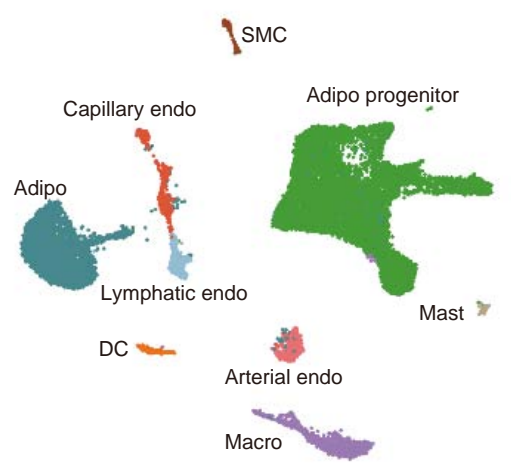

Salivary gland (74,461 nuclei)

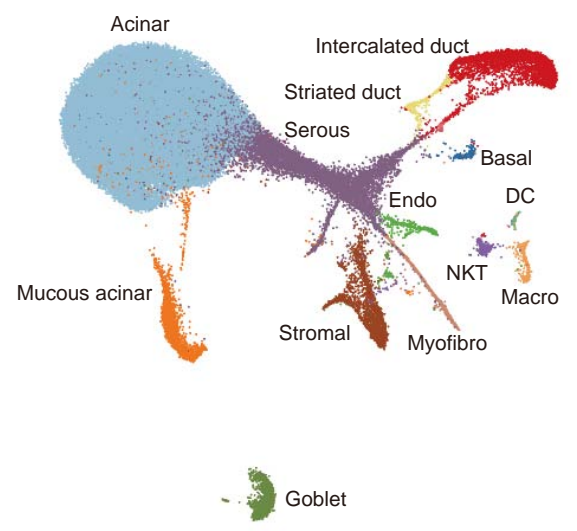

Spleen $(6,464$ cells)

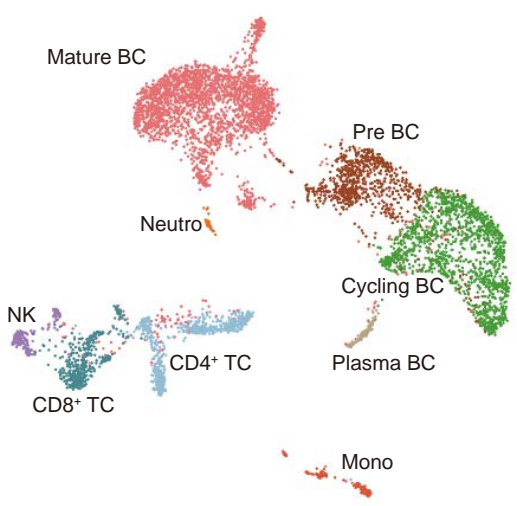

Substantia nigra (4,271 nuclei)

HTR2A+ IN,

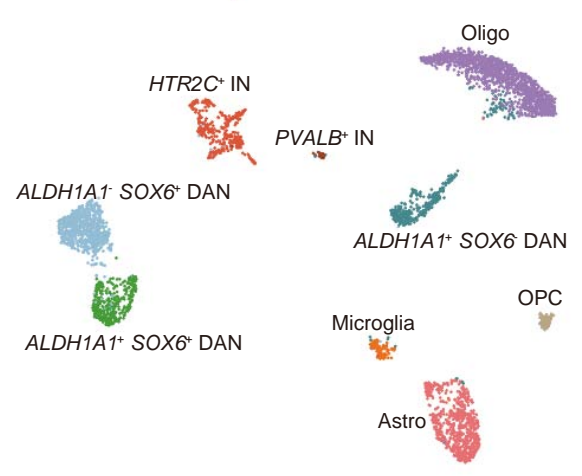


bioRxiv preprint doi: https://doi.org/10.1101/2021.12.13.472311: this version posted December 13, 2021. The copyright holder for this

preprint (which was not certified by peer review) is the author/funder, who has granted bioRxiv a license to display the preprint in

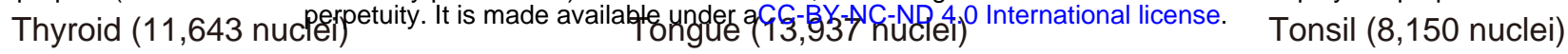

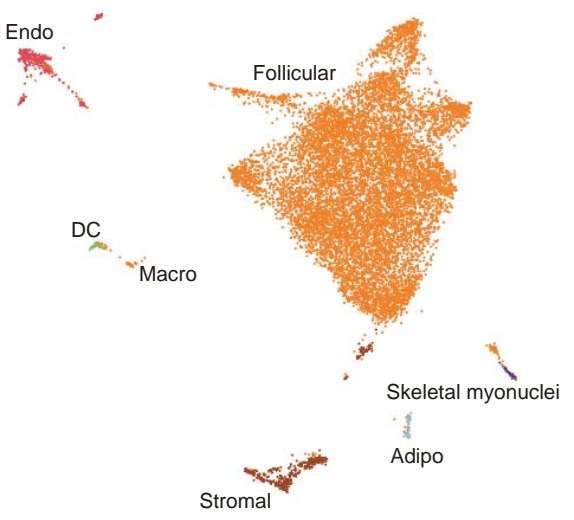

Trachea $(3,705$ nuclei)

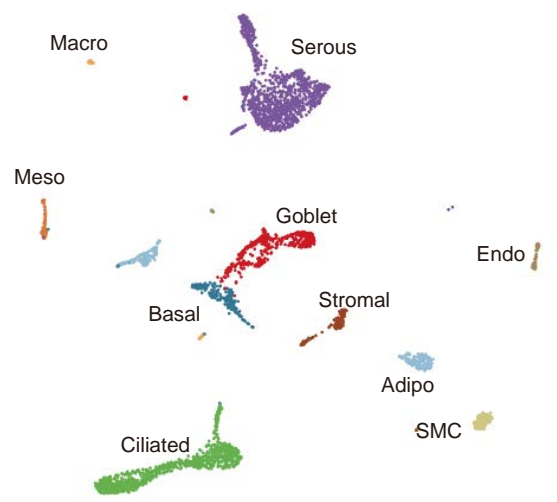

Visceral adipose (43,978 nuclei)

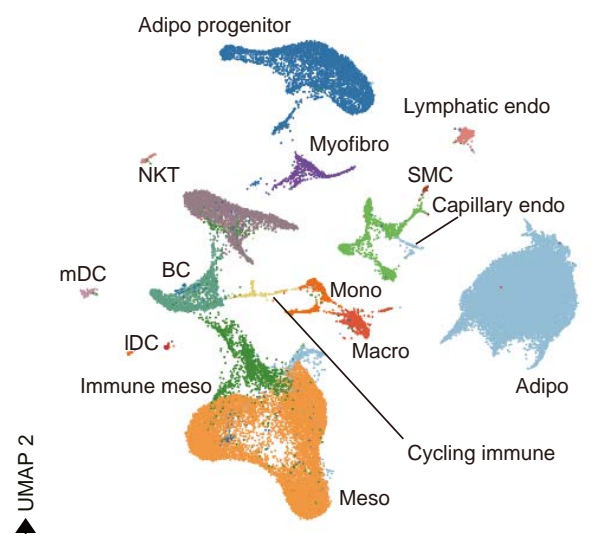

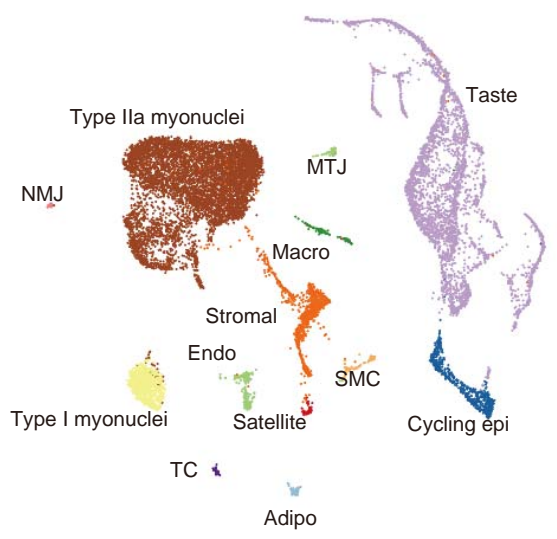

Uterus $(35,964$ nuclei)

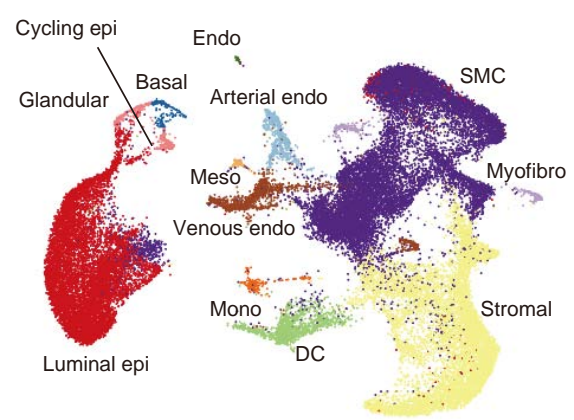

"Treg

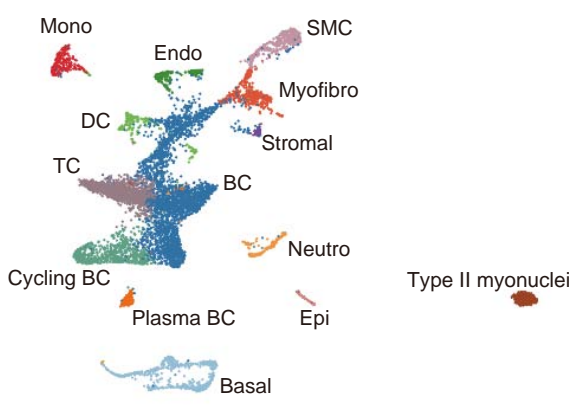

Vagina (2,265 nuclei)

Type Ilb myonuclei
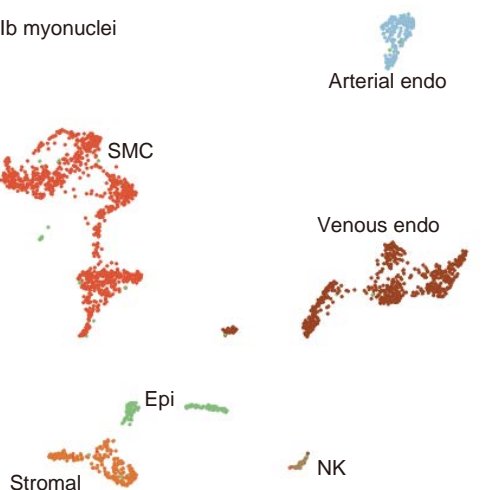
bioRxiv preprint doi: https://doi.org/10.1101/2021.12.13.472311; this version posted December 13, 2021. The copyright holder for this preprint (which was not certified by peer review) is the author/funder, who has granted bioRxiv a license to display the preprintiinoo

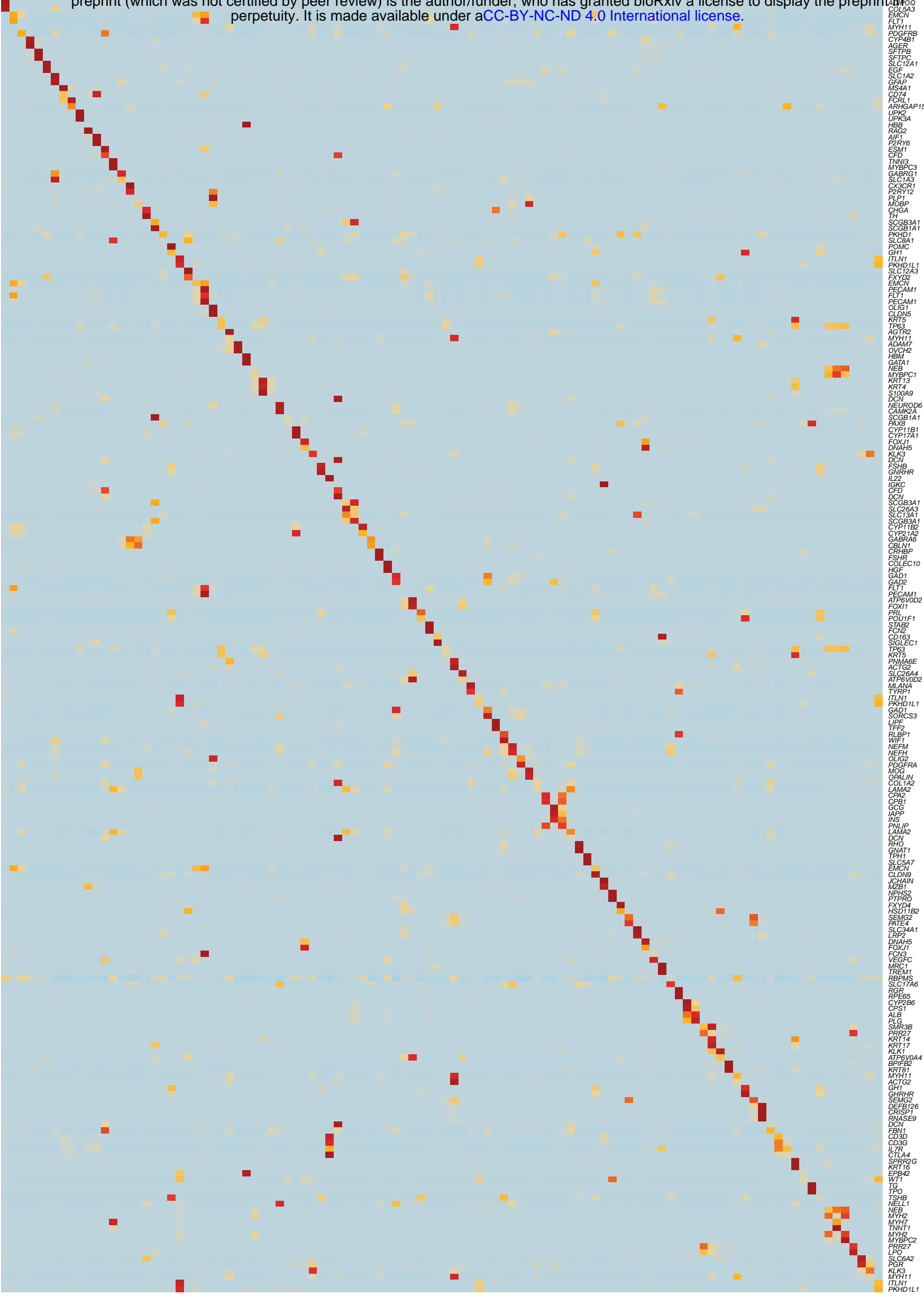

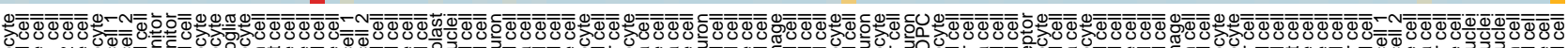

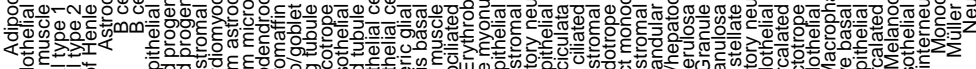

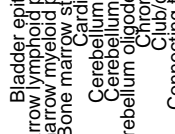

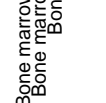

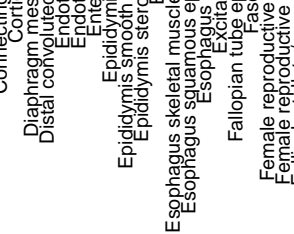

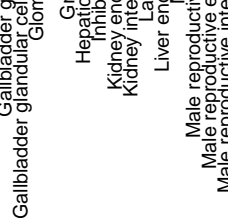

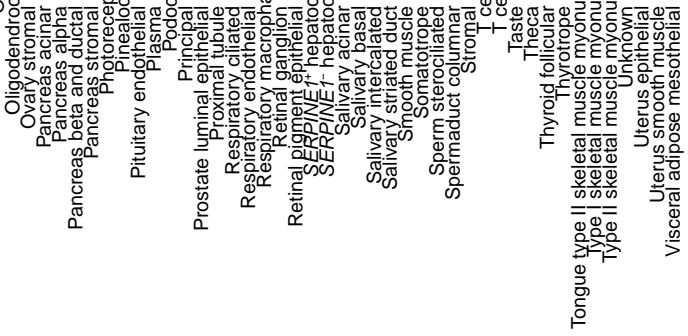

Z-score Zign Low High 
bioRxiv preprint doi: https://doi.org/10.1101/2021.12.13.472311; this version posted December 13,2021 . The copyright holder for this
preprint (which was not certified by peer review) is the author/funder, who has granted bioRxiv a license to display the preprint in
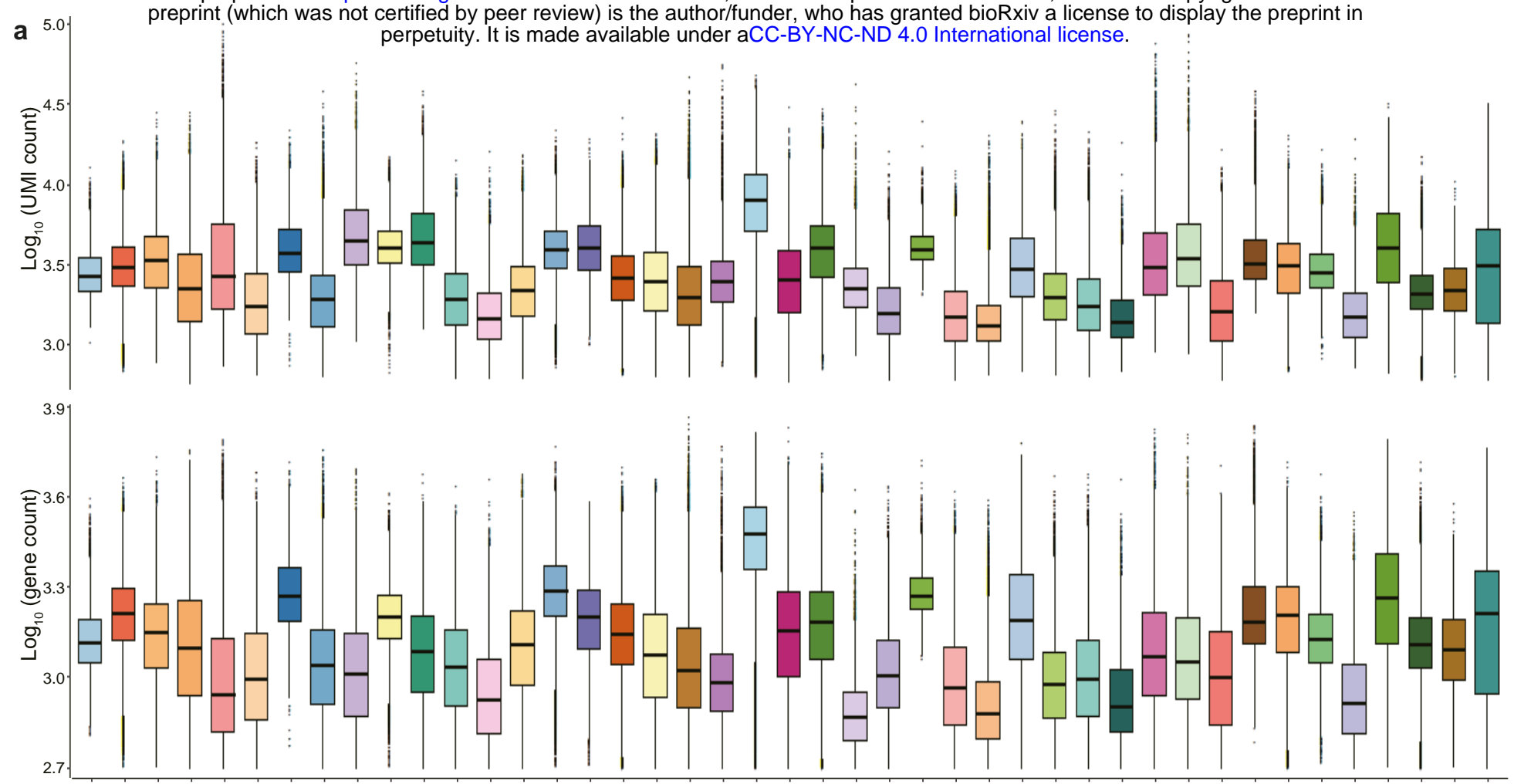

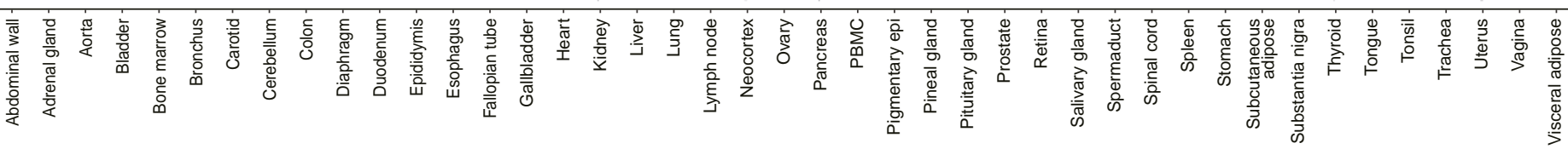
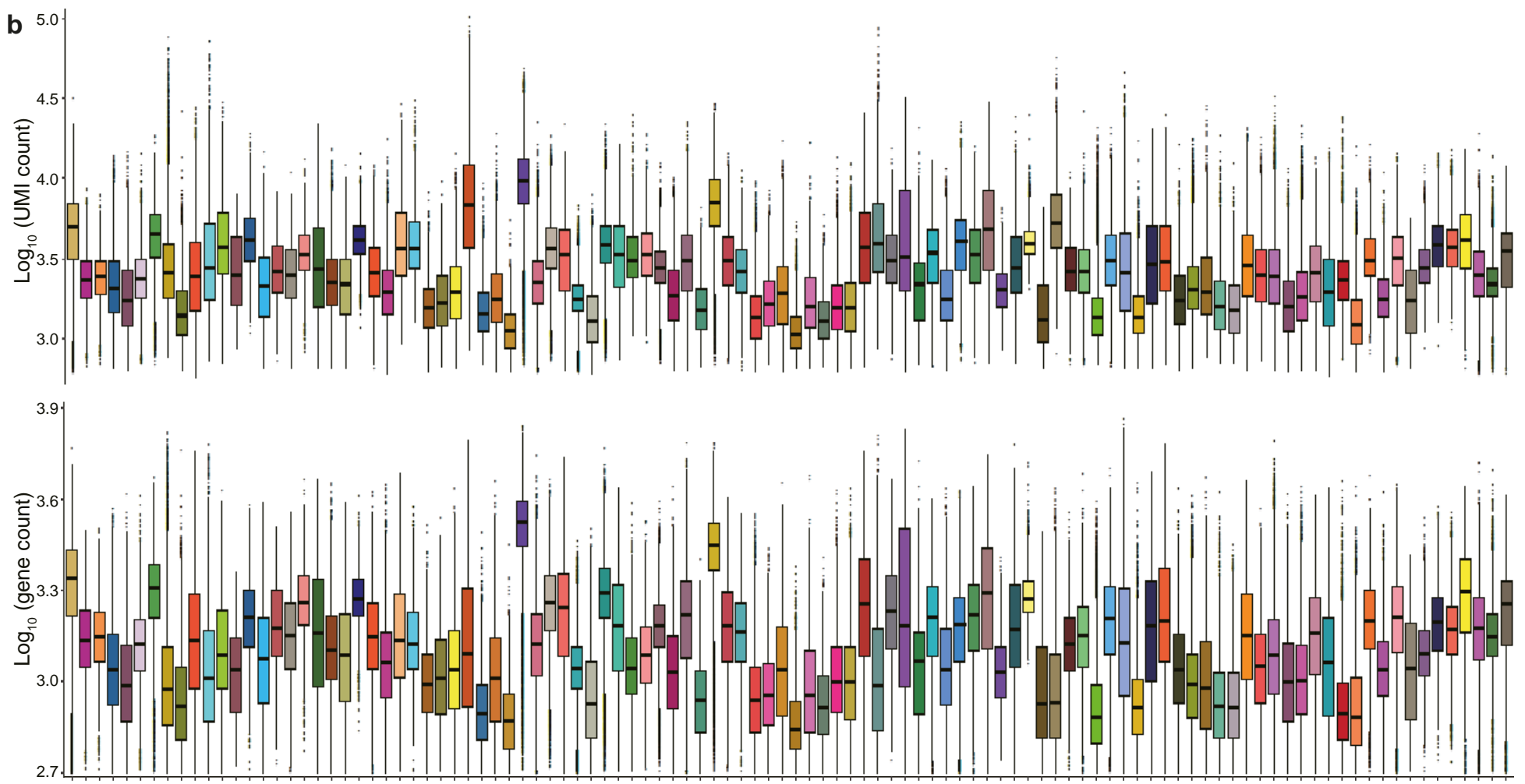

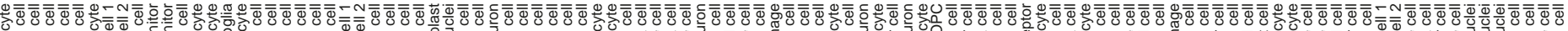

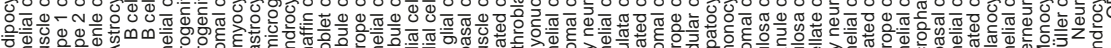

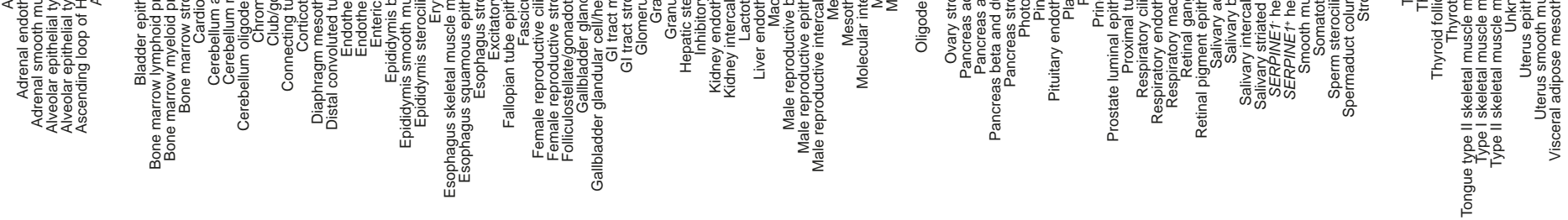


bioRxiv preprint doi: https://doi.org/10.1101/2021.12.13.472311; this version posted December 13, 2021. The copyright holder for this preprint (which was not certified by peer review) is the author/funder, who has granted bioRxiv a license to display the preprint in
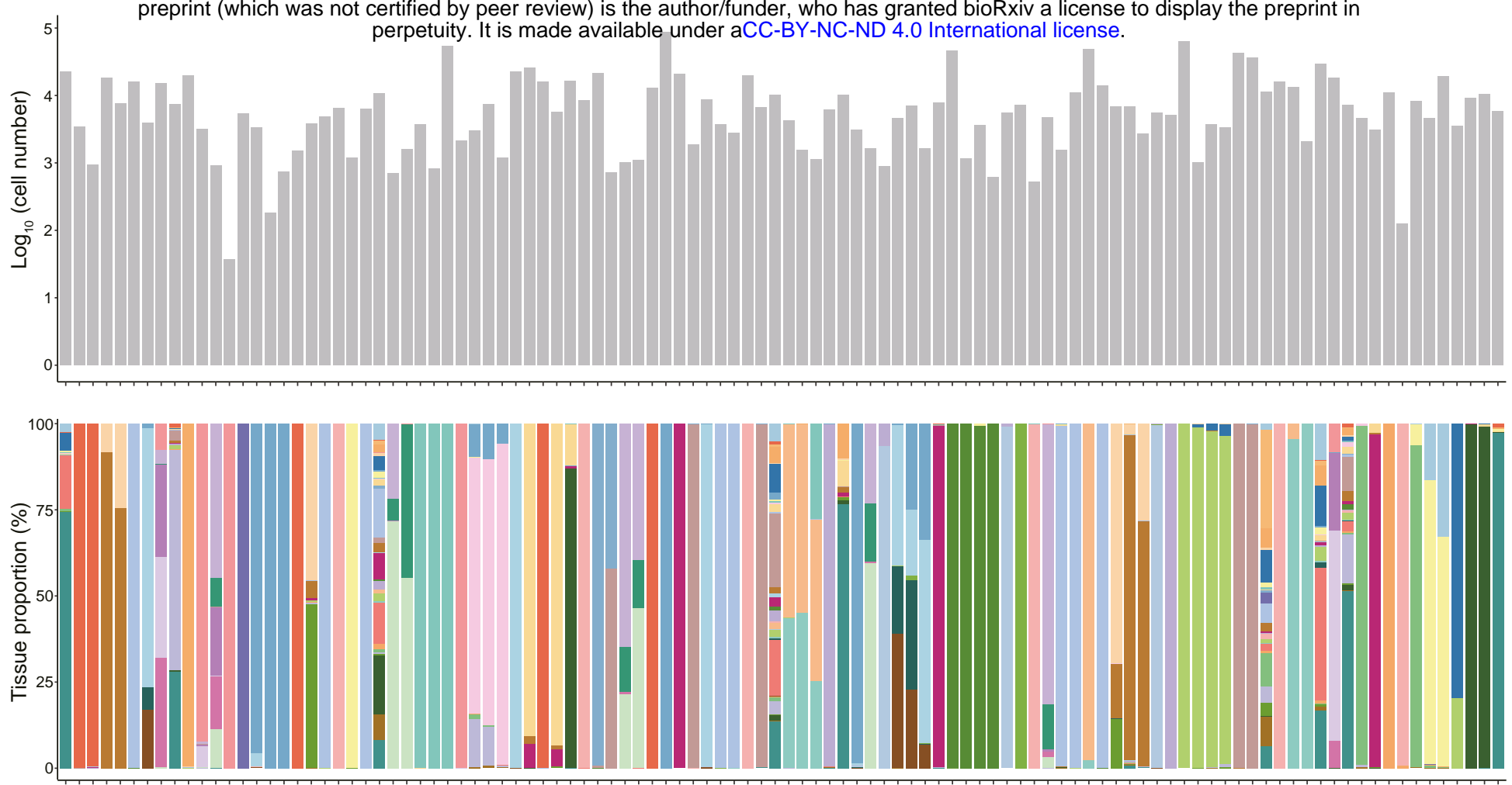

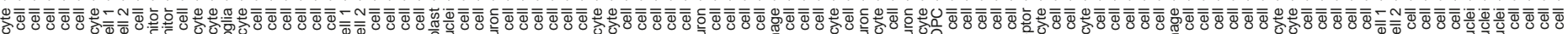

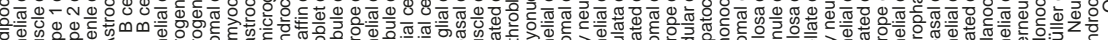

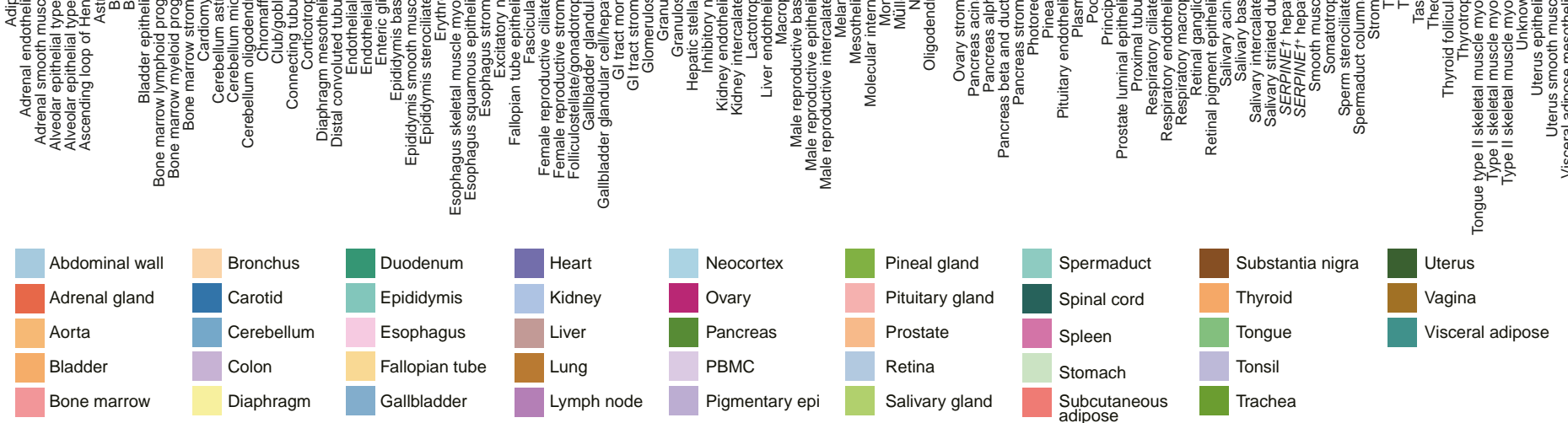




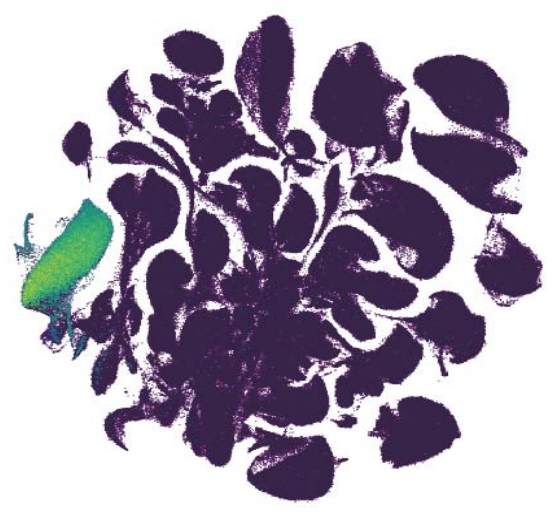

ROS1 (epididymis sterociliated cell)

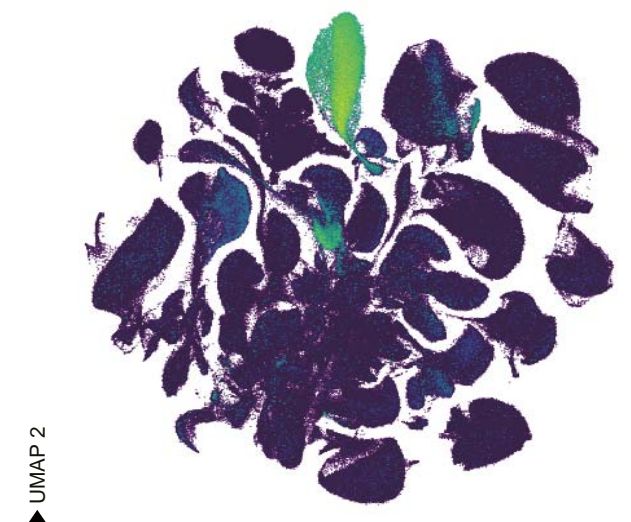

b

FLT1 (endothelial cell)

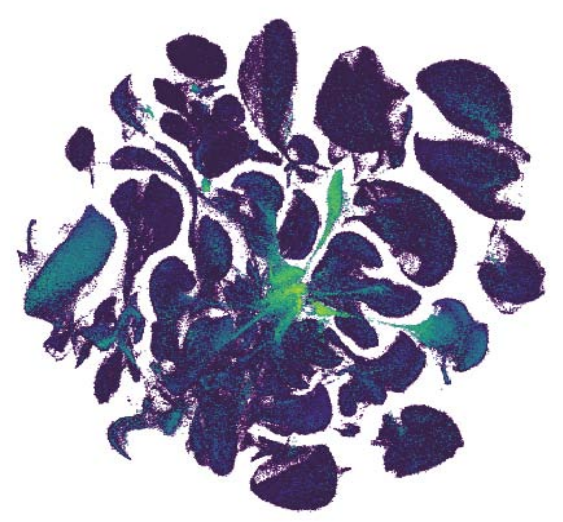

TTN (skeletal myonuclei)

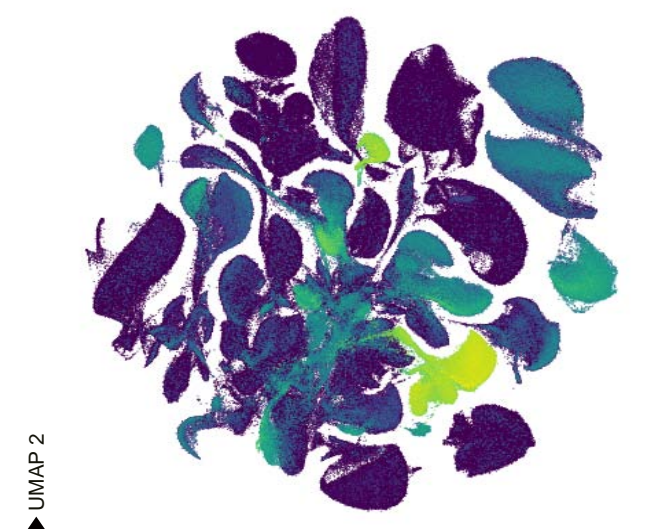

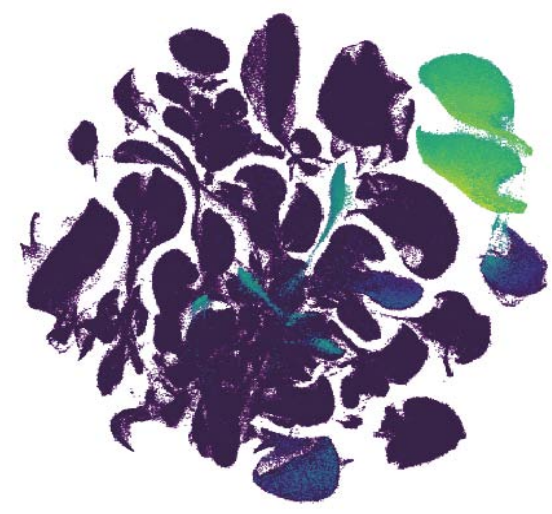

GCG (pancreas alpha cell)

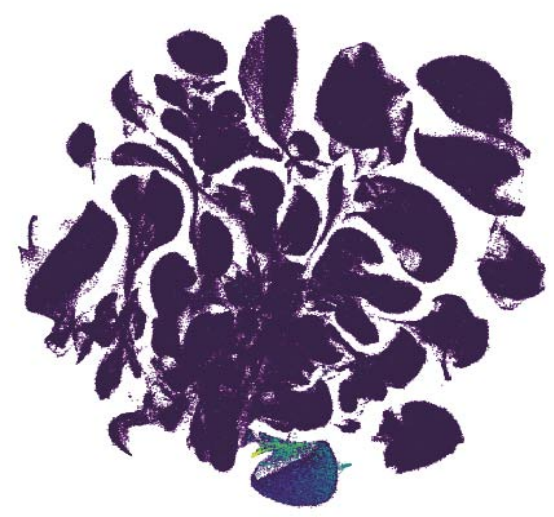

DCN (stromal cell)

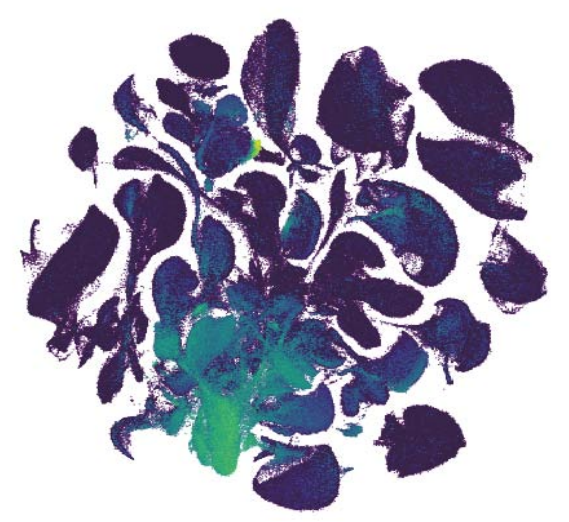

ADIPOQ (adipocyte)

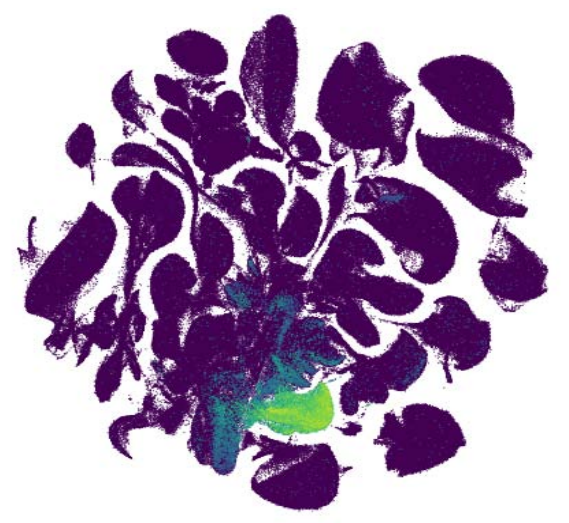

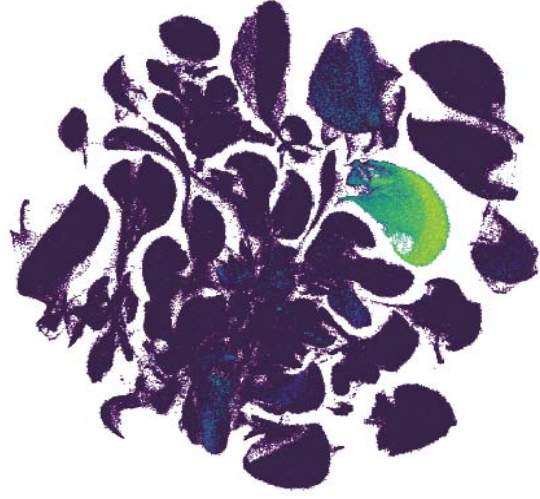

CYP11B1 (fasciculata cell)

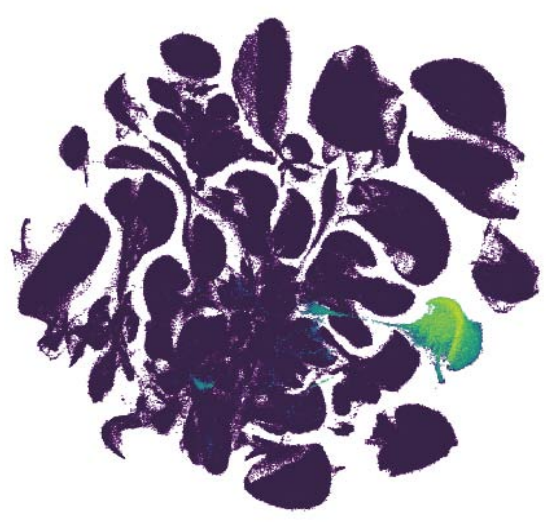

PTPRC (immune cell)

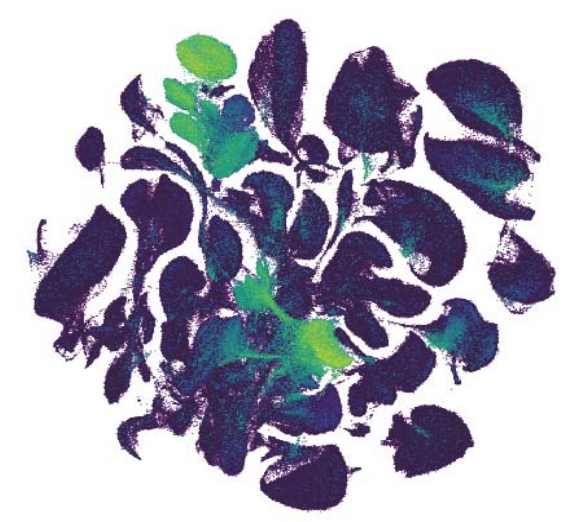

ITLN1 (mesothelial cell)

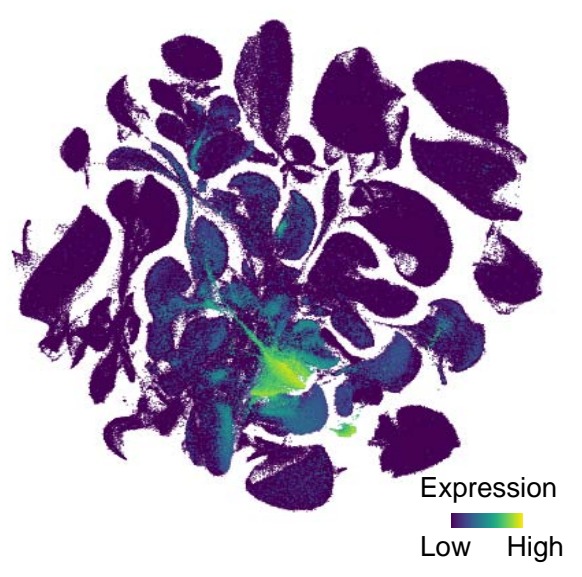


bioRxiv preprint doi: https://doi.org/10.1101/2021.12.13.472311; this version posted December 13,2021. The copyright holder for this preprint (which was not certified by peer review) is the author/funder, who has granted bioRxiv a license to display the preprint in
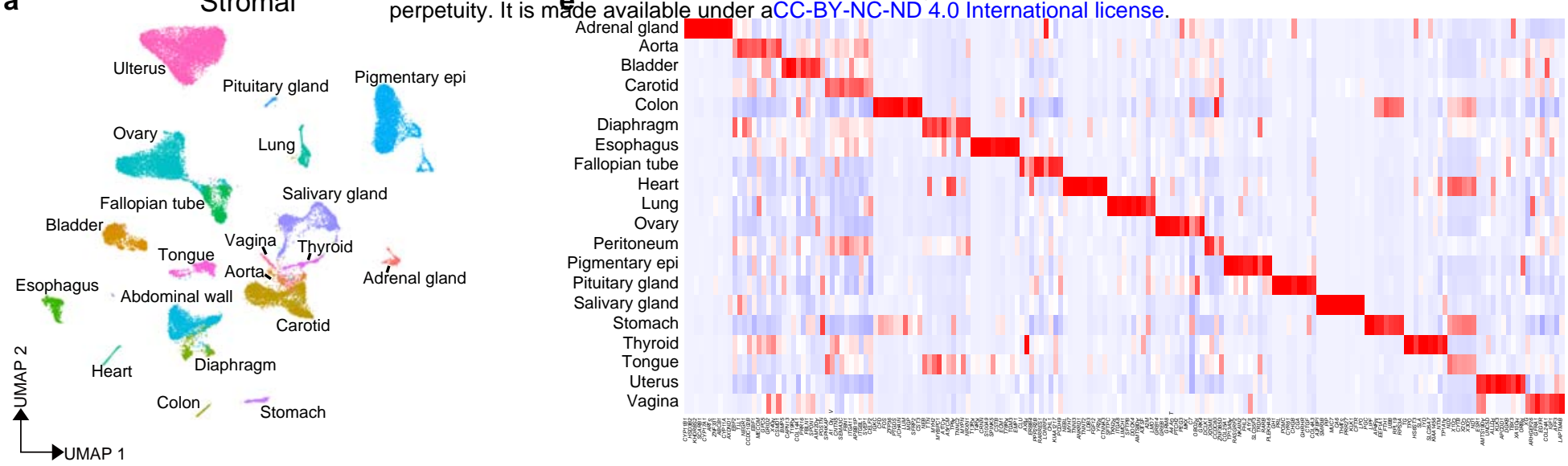

b

Macrophage
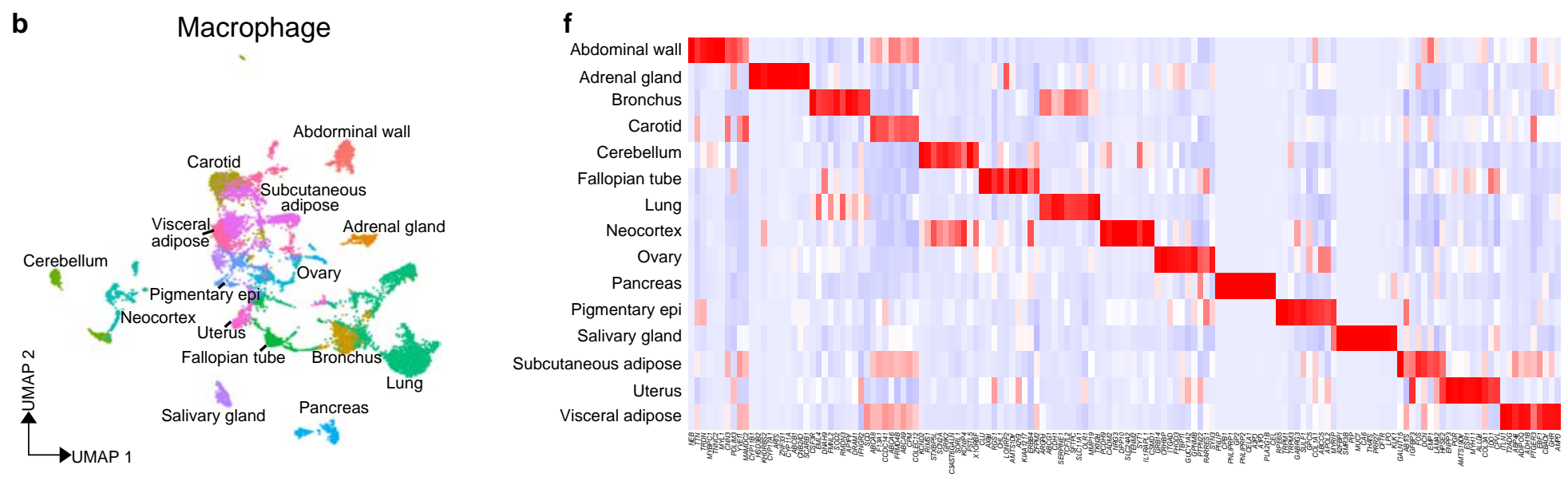

C

\section{Endothelial}

g

Gallbladder

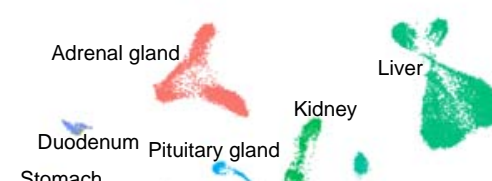

Stomach

Stomach Salivary gland Thyroid
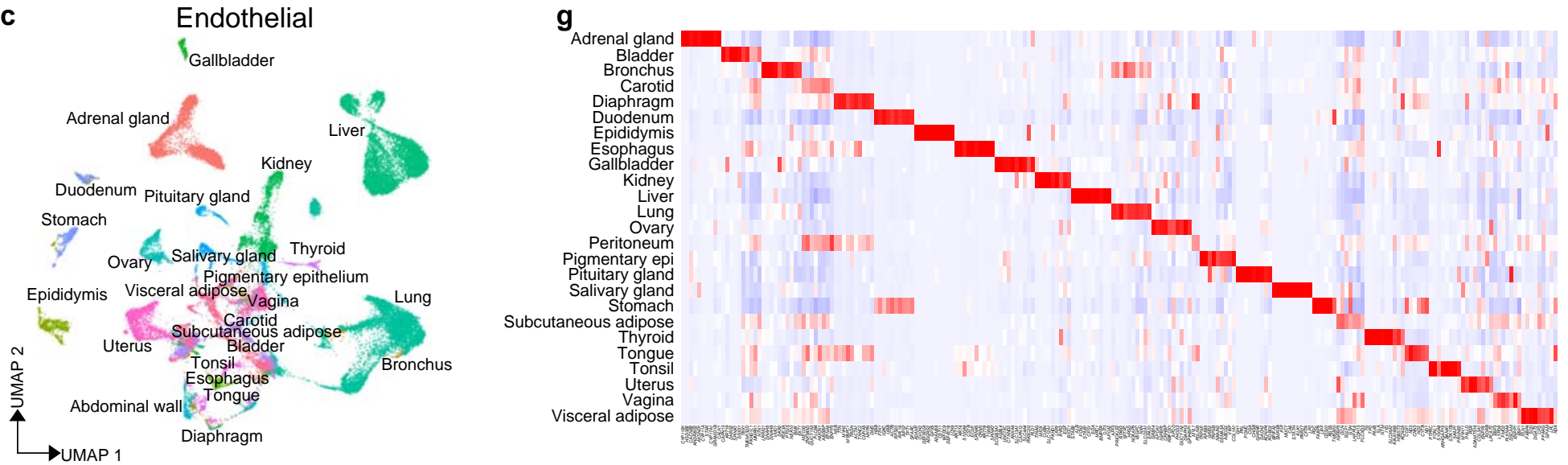

d

Smooth muscle
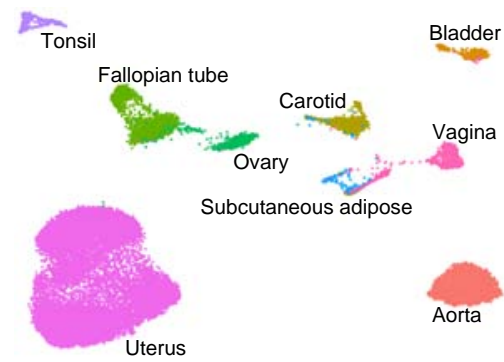

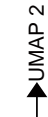

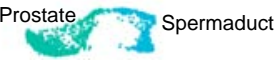

h

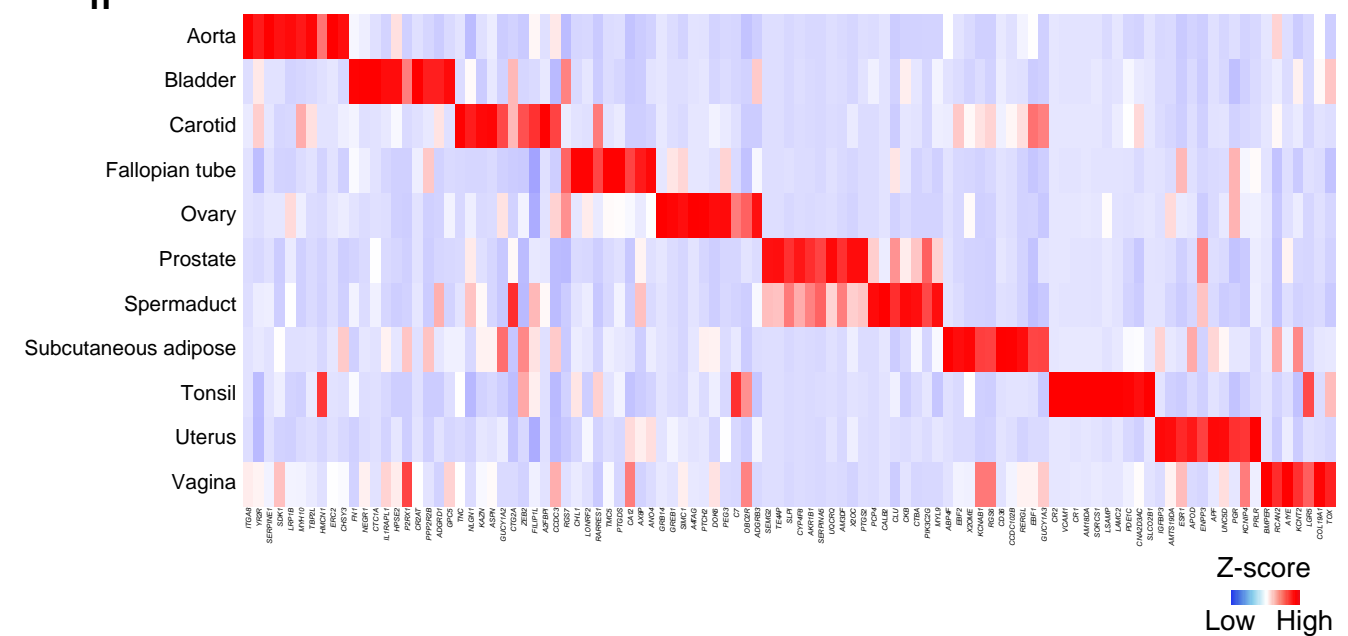


bioRxiv preprint doi: https://doi.org/10.1101/2021.12.13.472311; this version posted December 13, 2021. The copyright holder for this preprint (which was not certified by peer review) is the author/funder, whohas granted bioRxiv a license to display the preprint in

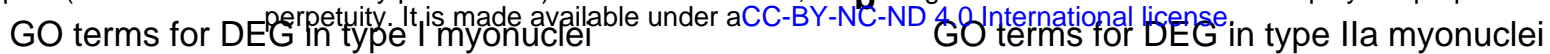

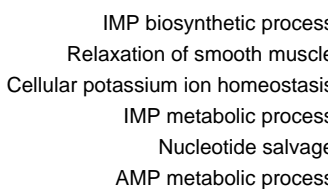

Purine-containing compound salvage Negative regulation of smooth muscle contraction Cell volume homeostasis

Negative regulation of muscle contraction Cellular response to extracellular stimulus Regulation of type B pancreatic cell proliferation Cellular response to external stimulus Type B pancreatic cell proliferation Regulation of myoblast proliferation Myoblast proliferation Muscle system process

Positive regulation of epithelial cell proliferation Fat cell differentiation

Vascular associated smooth muscle cell migration Positive regulation of sodium ion transport Actin-mediated cell contraction Actin filament-based movement

Regulation of sodium ion transmembrane transporter activity Regulation of sodium ion transmembrane transport Positive regulation of sodium ion transmembrane transporter activity Regulation of sodium ion transport Positive regulation of sodium ion transmembrane transport Synapse organization

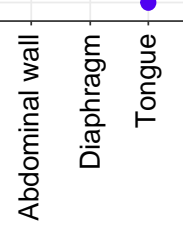

GO terms for DEG in type Ilb myonuclei

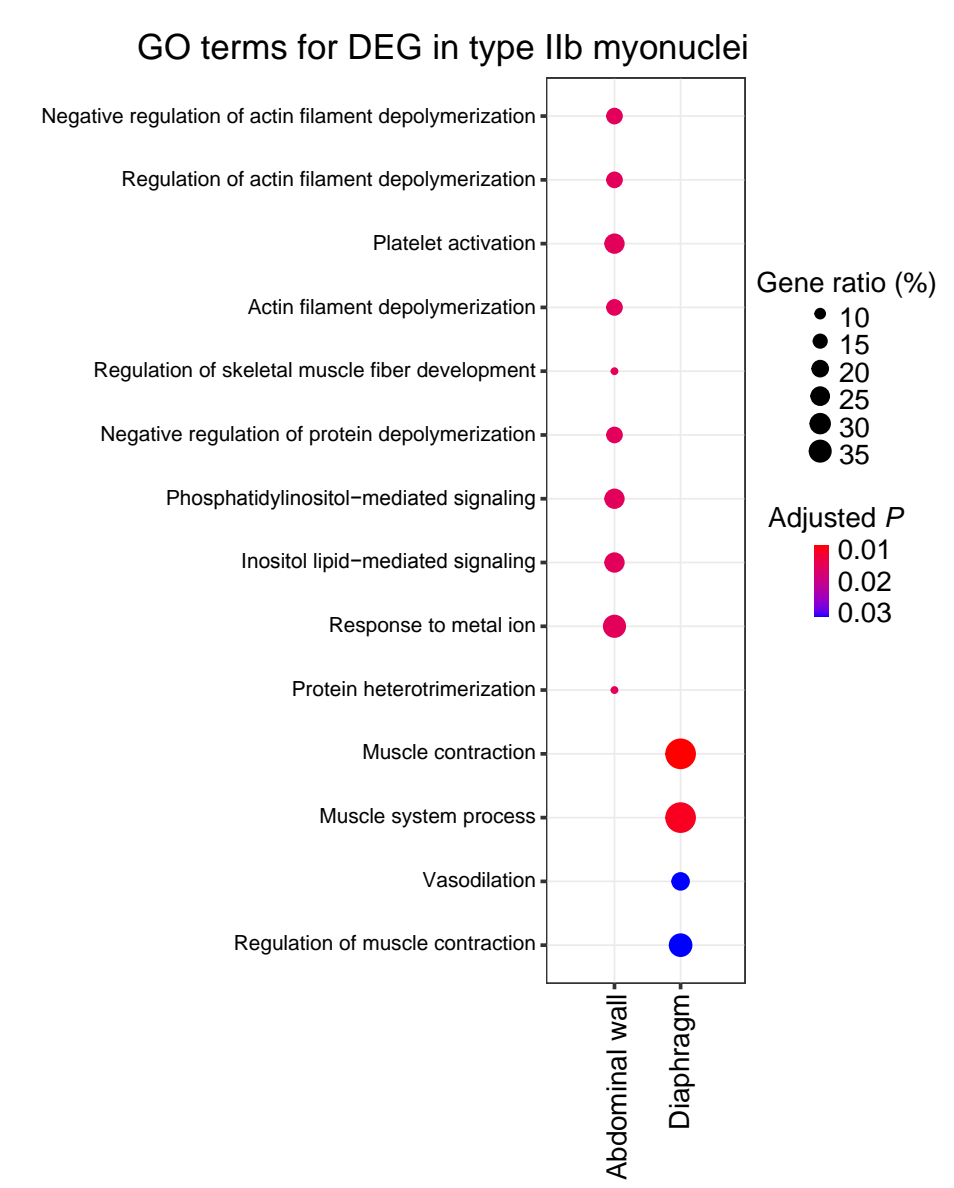

C
Gene ratio (\%)

- 10

15

20
2
0

Adjusted $P$

0.02

0.03

0.04

Mitochondrial AT

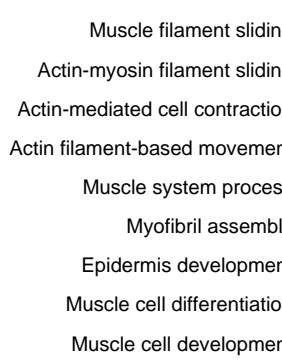

Cellular component assembly involved in morphogenesis

Multicellular organismal movement

Musculoskeletal movement

Respiratory electron transport chain

Electron transport chain

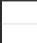

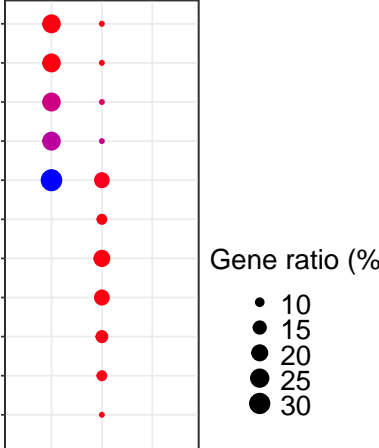

Adjusted $P$

0.01

0.02

0.03 


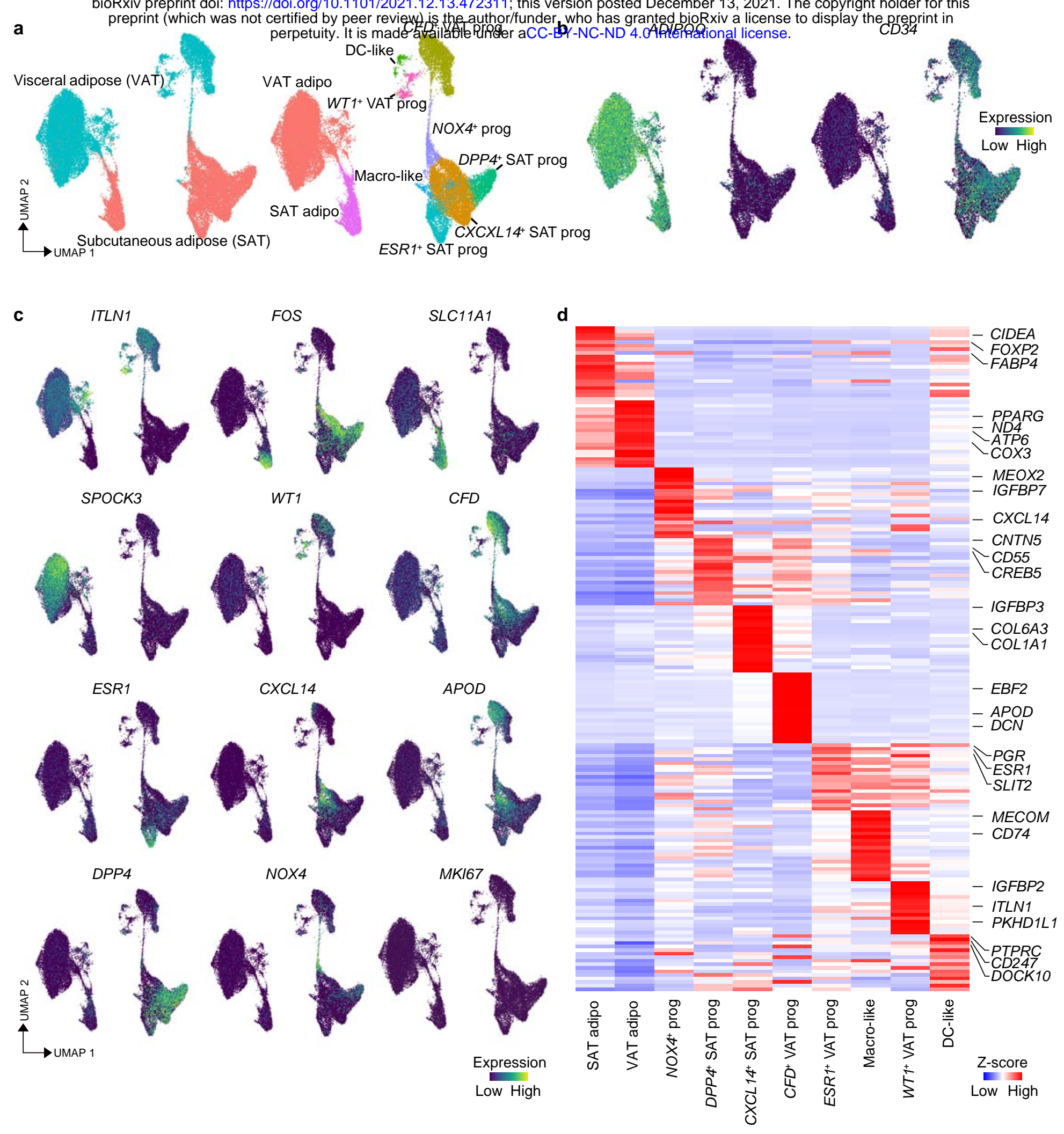


bioRxiv preprint doi: https://doi.org/10.1101/2021.12.13.472311; this version posted December 13, 2021. The copyright holder for this preprint (which was not certified by peer review) is the author/funder, who has granted bioRxiv a license to display the preprint in

a LGR6

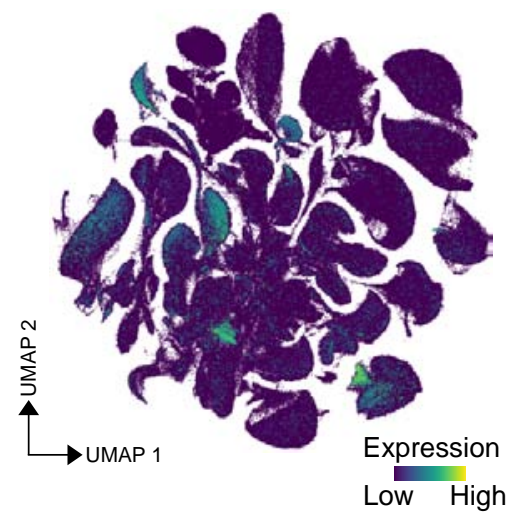
perpetuity. It is made available under aCC-BC-NC-ND 4.0 InteตRational license.
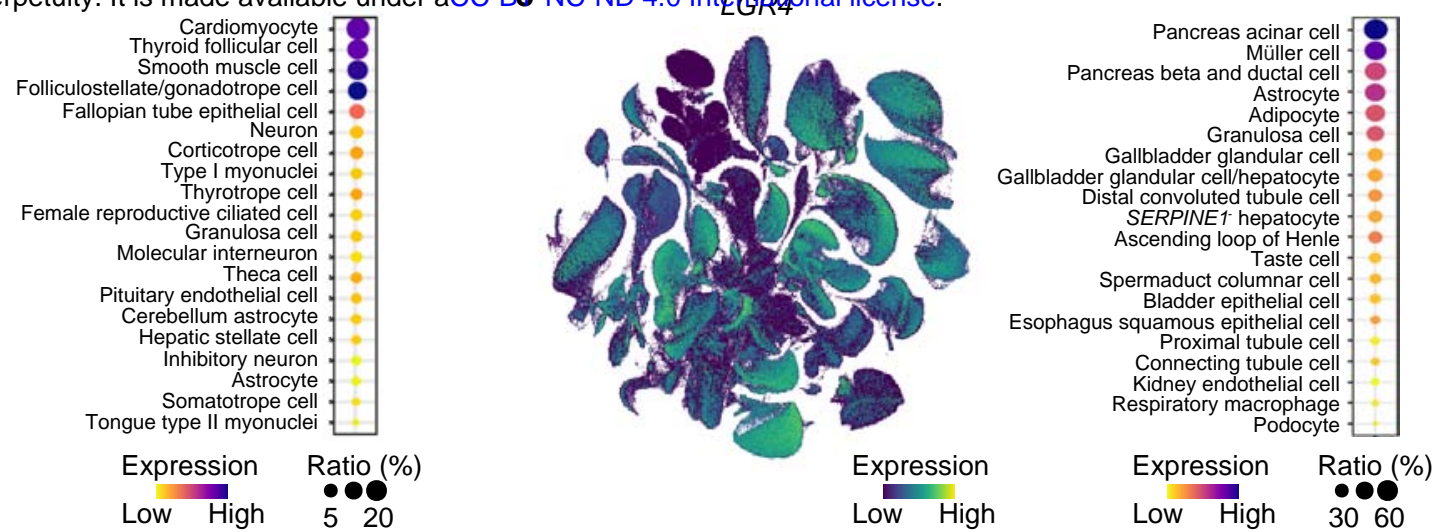

b LGR5 and LGR6 co-expressed

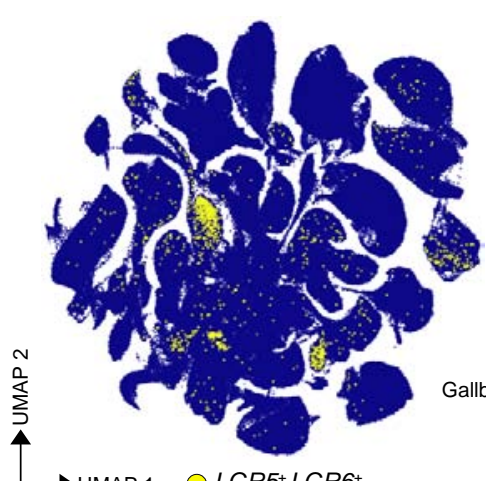

$L G R 5^{+} L G R 6^{+}$

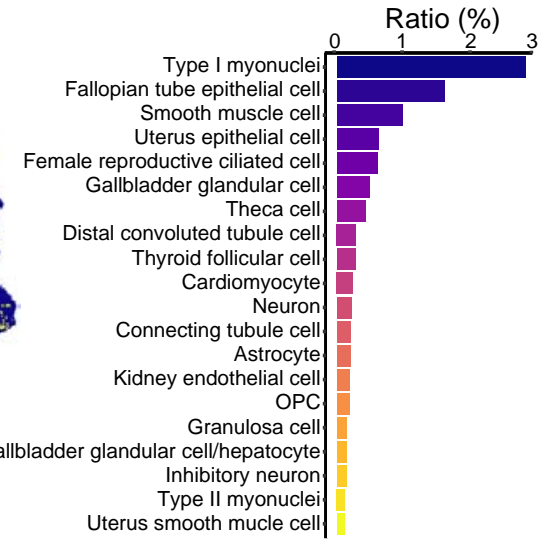


bioRxiv preprint doi: https://doi.org/10.1101/2021.12.13.472311; this version posted December 13,2021 . The copyright holder for this preprint (which was not certified by peer review) is the author/funder who has granted bjoBxiv a license to djsplay the preprint in

Expression

Low High

Abdominal wall

Adrenal gland

Aorta

Bone marrow

Cerebellum

Diaphragm

Duodenum

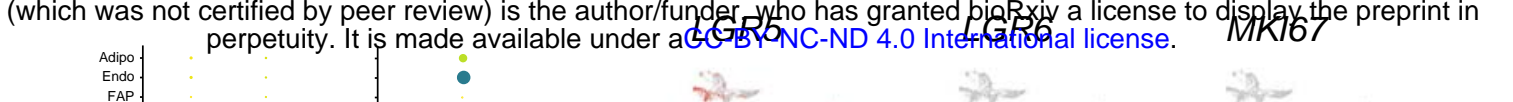


bioRxiv preprint doi: https://doi.org/10.1101/2021.12.13.472311; this version posted December 13, 2021. The copyright holder for this preprint (which was not certified by peer review) is the author/funder who has granted bjoBxiv a license to djsplay the preprint in Expression

Low High

Epididymis

Fallopian tube

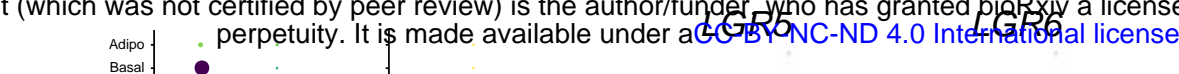

Esophagus

Gallbladder
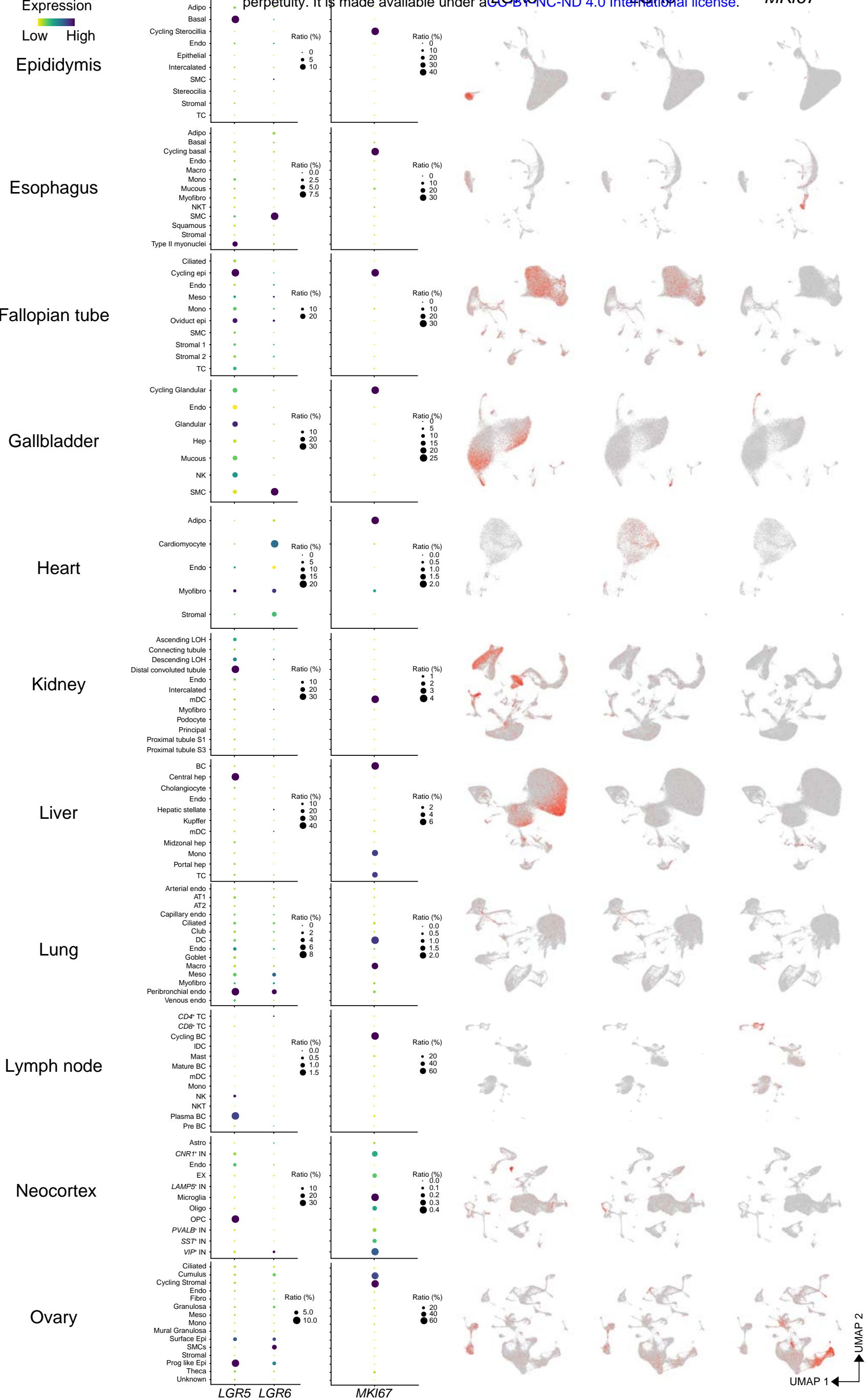

Expression Low High 
bioRxiv preprint doi: https://doi.org/10.1101/2021.12.13.472311; this version posted December 13, 2021. The copyright holder for this preprint (which was not certified by peer review) is the author/funder who has granted biokiviv a license to djsplak the preprint in
Expression

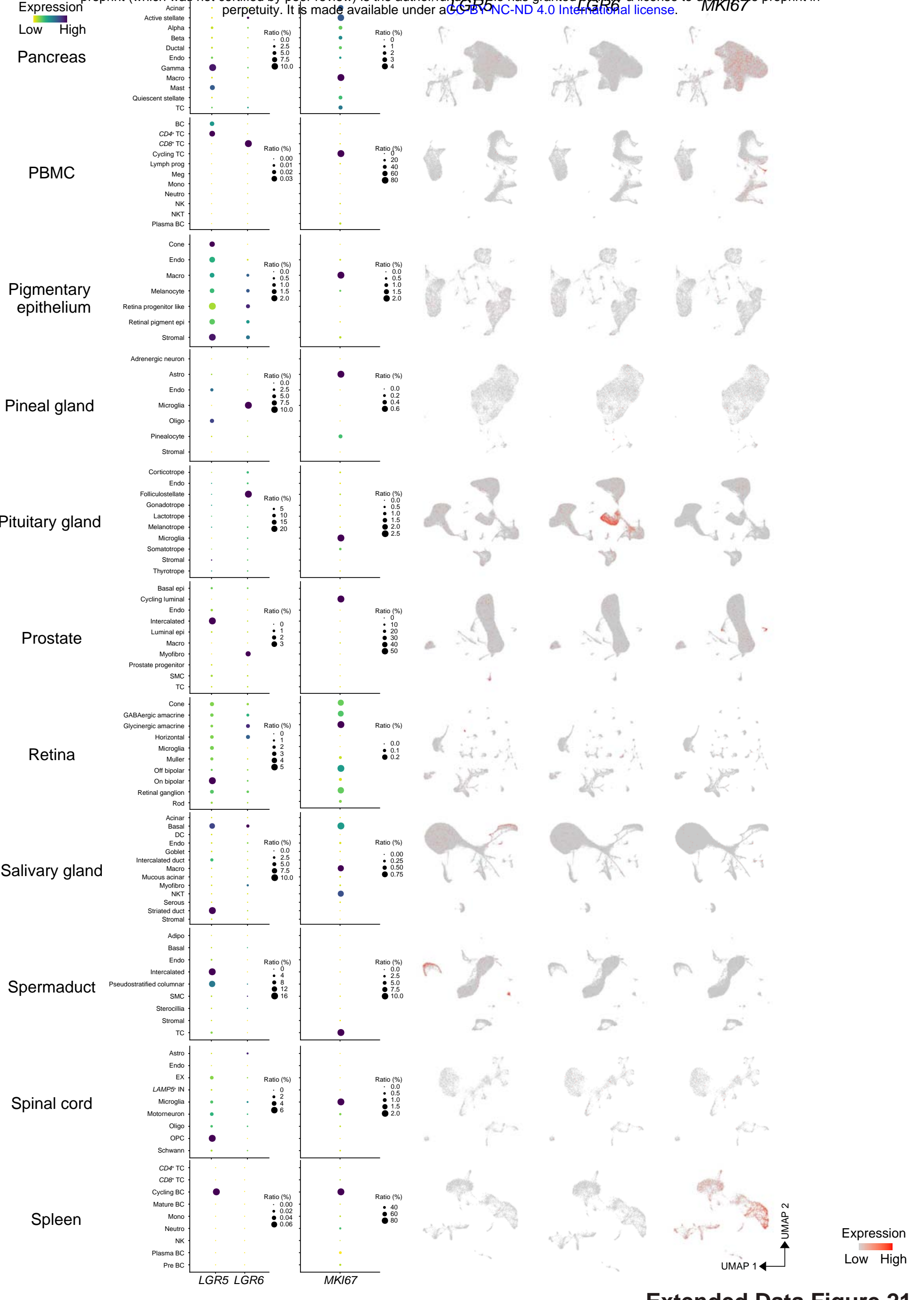


bioRxiv preprint doi: https://doi.org/10.1101/2021.12.13.472311; this version posted December 13, 2021. The copyright holder for this preprint (which was not certified by peer review) is the author/funder who has granted bjoBxiv a license to djsplay the preprint in

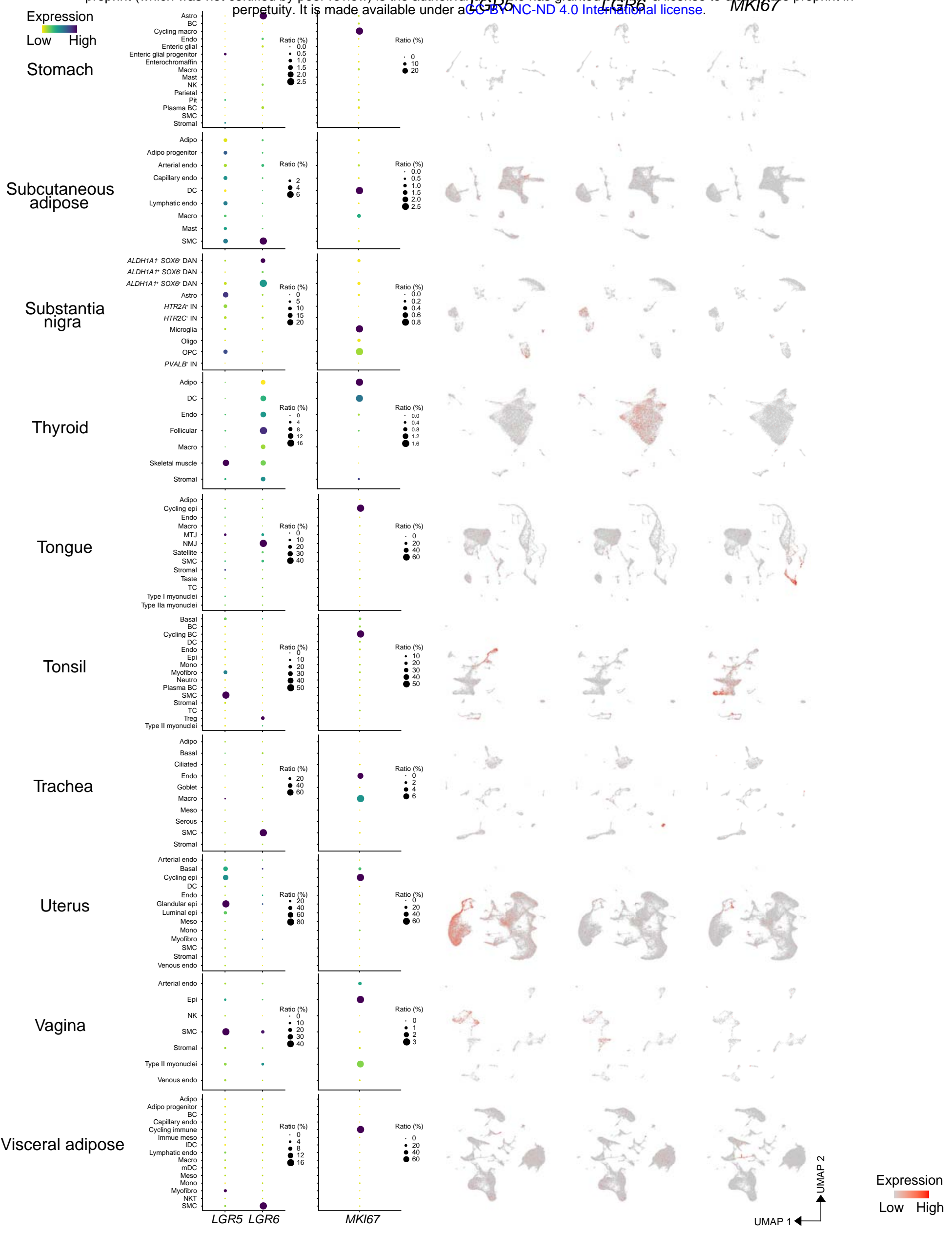


bioRxiv preprint doi: https://doi org/10.1101/2021.12.13.472311; this version posted December 13, 2021. The copyright holder for this preprint (which was not certified by peer review) is the author/funder, who has granted bioRxiv a license to display the preprint in

$$
\text { snRNA-seq }
$$
perpetuity. It is made available under aCC-BY-NC-ND 4.0 International license.

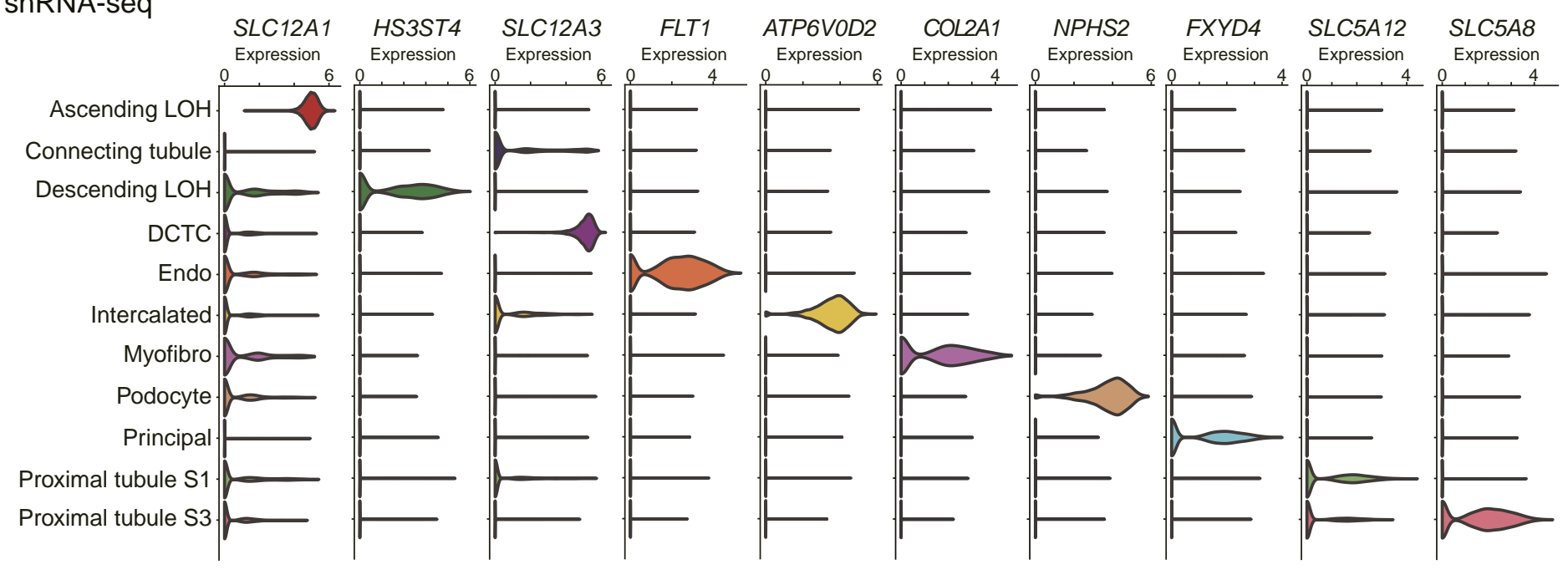

b

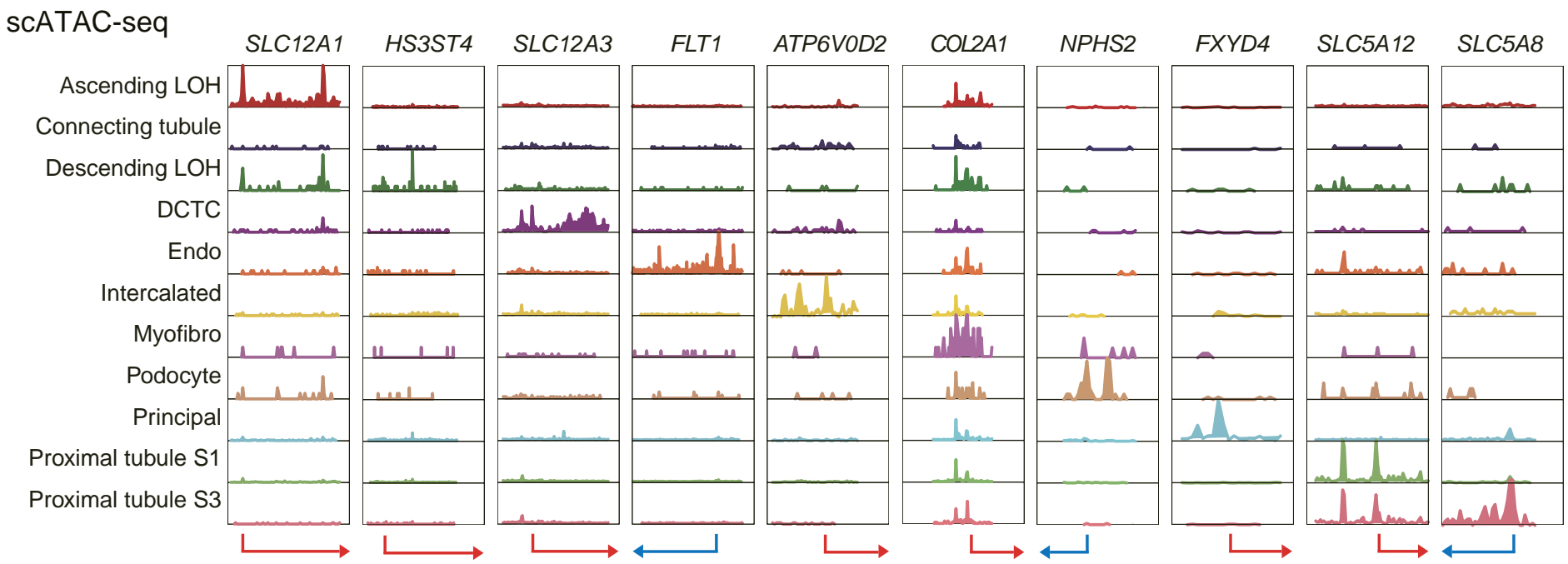


bioRxiv preprint doi: https://doi.org/10.1101/2021.12.13.472311; this version posted December 13, 2021. The copyright holder for this preprint (which was not certified by peer review) is the author/funder, who has granted bioRxiv a license to display the preprint in perpetuity. It is made available under aCC-BY-NC-ND 4.0 International license.
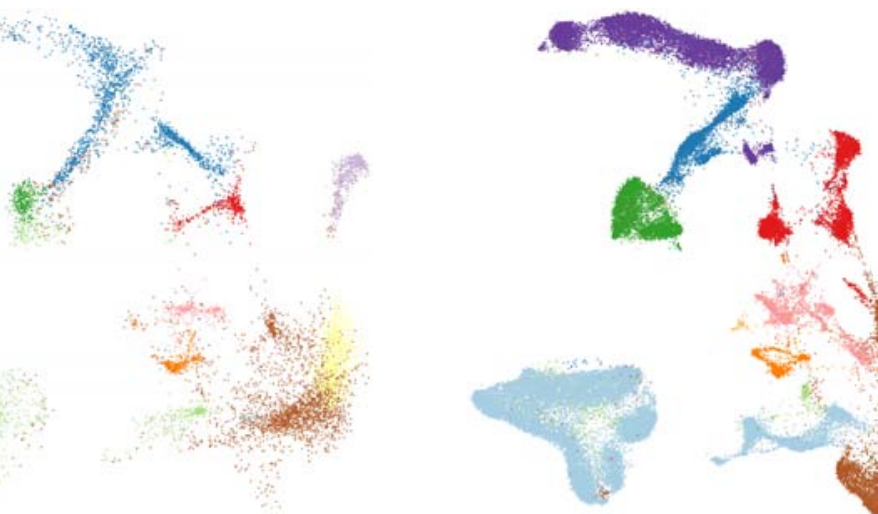

Ascending $\mathrm{LOH}$

Descending LOH Endo

- Intercalated

mDC

Podocyte

- Principal

Proximal tubule S1

- Connecting tubule DCTC

LGR5 (human kidney)

LGR5 (monkey kidney)
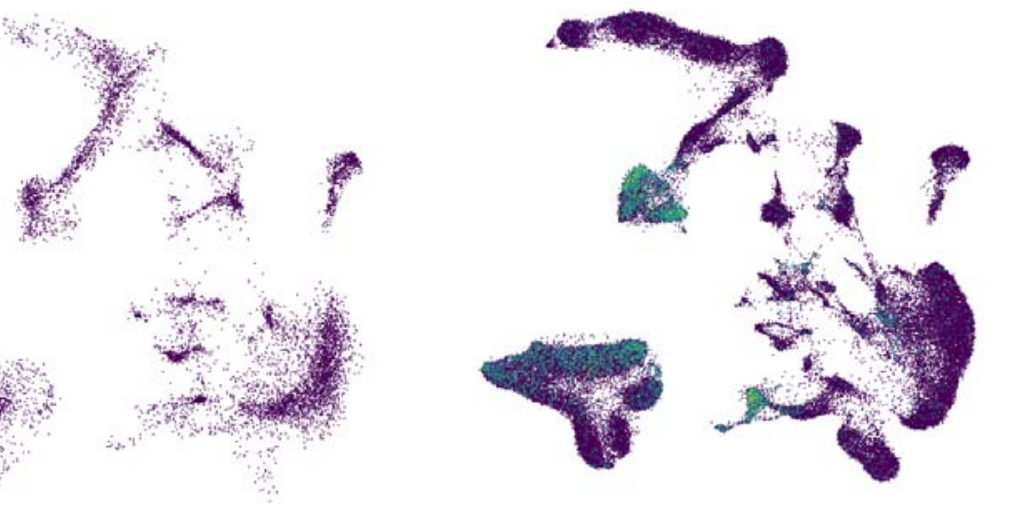

C
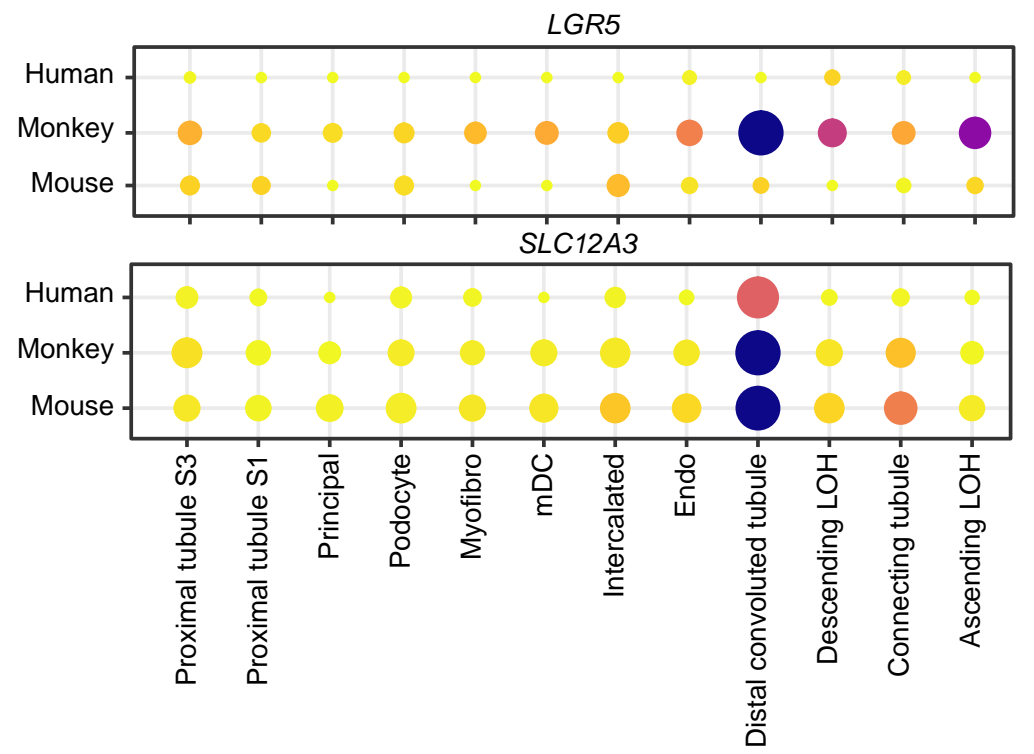

LGR5 (mouse kidney)<smiles>CC#CC#[As]</smiles>

Expression Low High

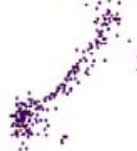

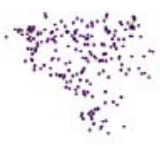

Ratio (\%)

10

20

30

25

50

75

Expression

Low High 

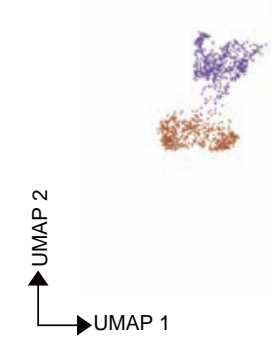

b

c

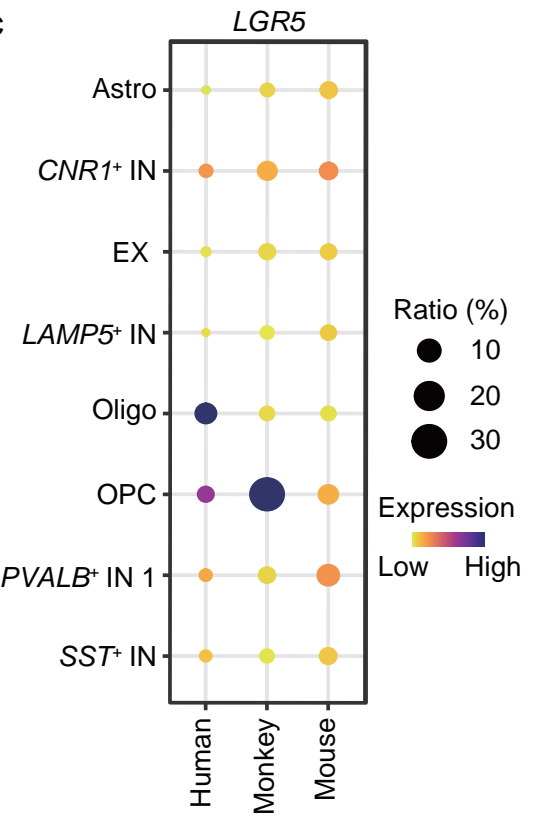

d

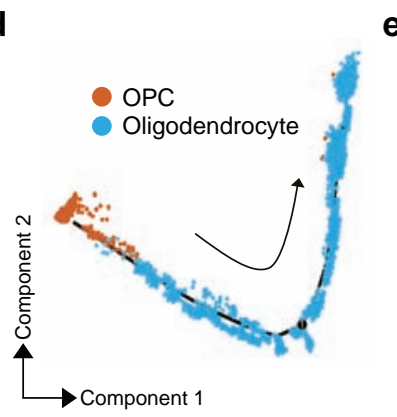
LGR5 (human neocortex)

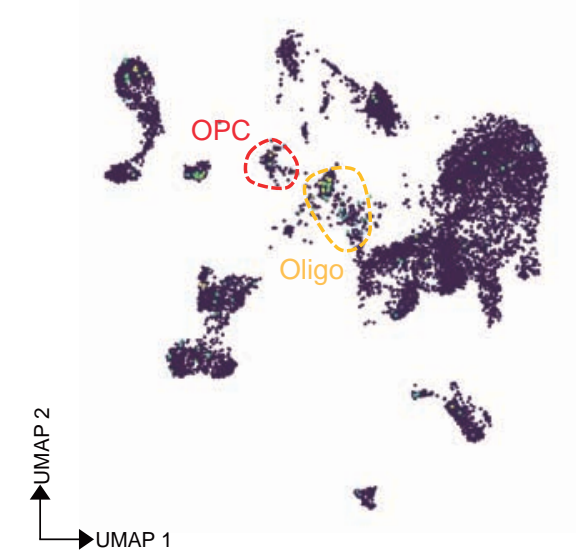

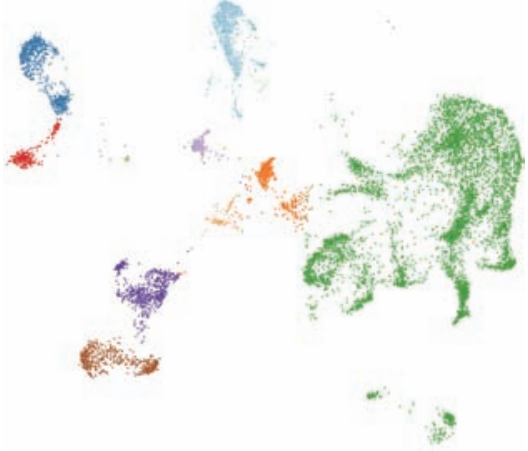

QY.

$$
\begin{array}{ll}
\text { Astro } & \text { Endo } \\
\text { CNR1+ IN } & \text { EX }
\end{array}
$$
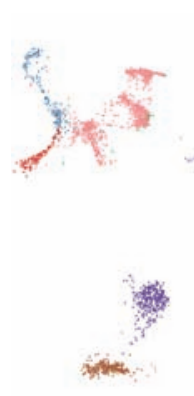

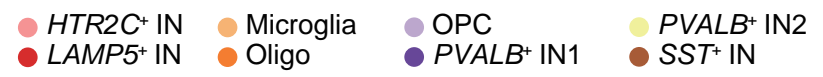

LGR5 (monkey neocortex)
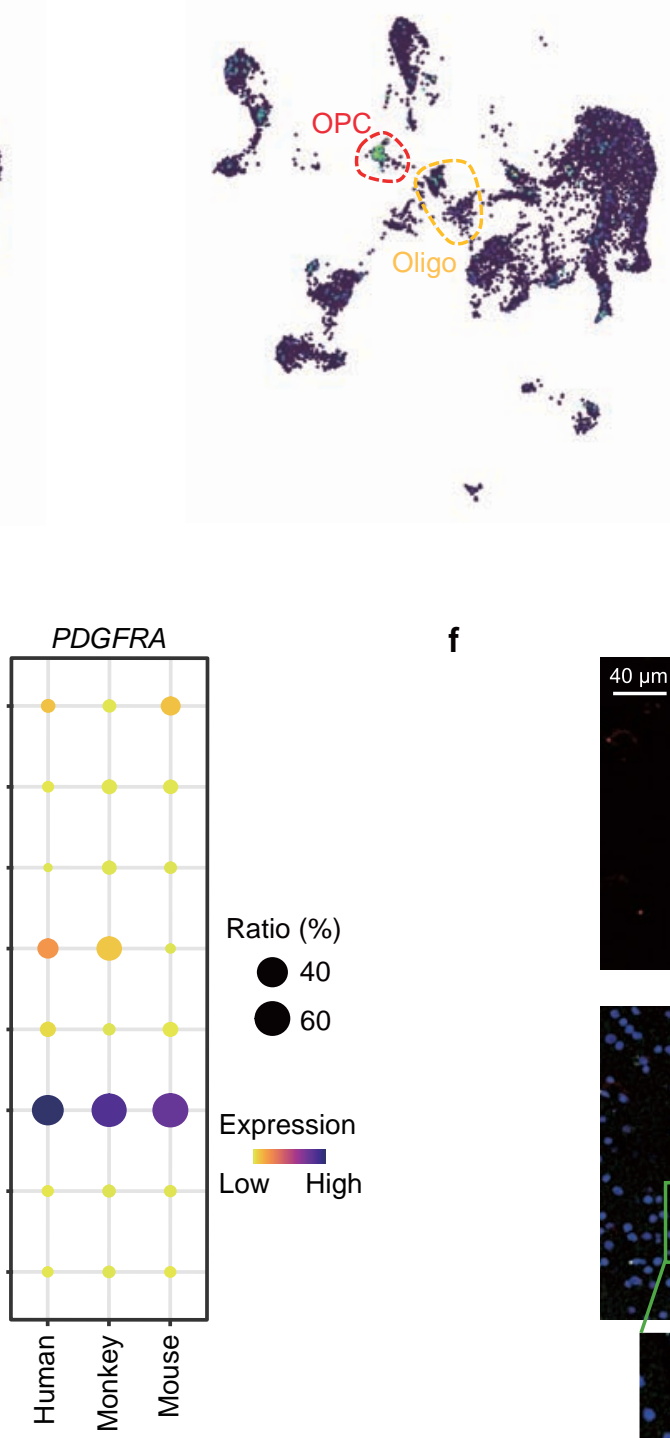

LGR5 (mouse neocortex)

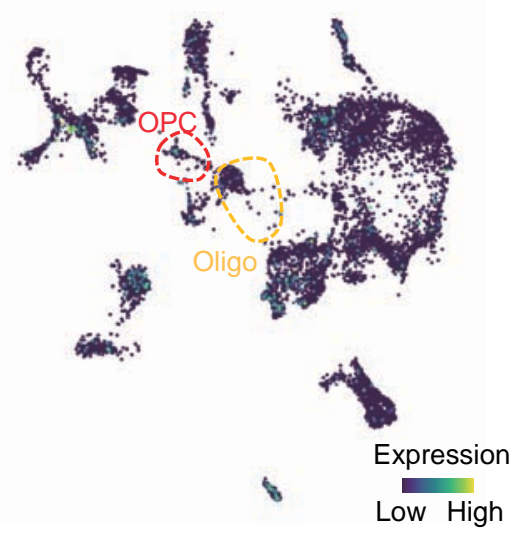

f
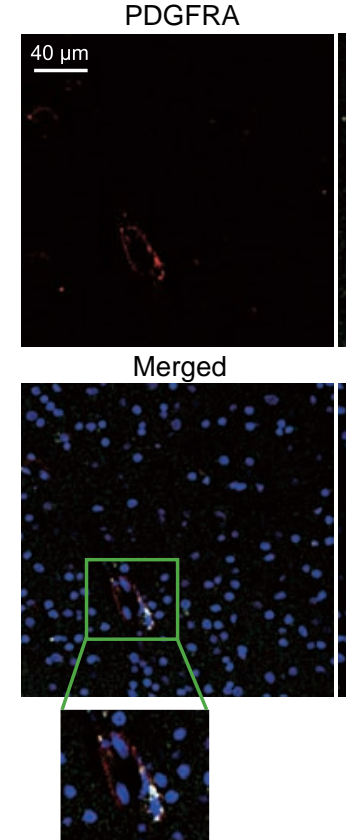

e

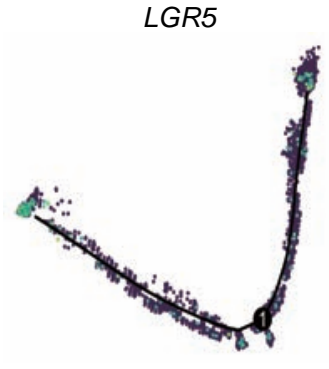

OLIG2

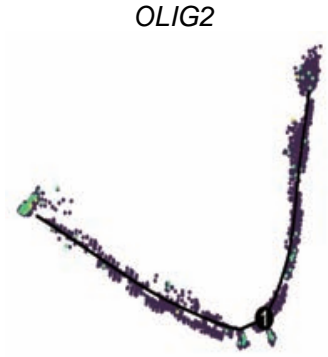

PDGFRA

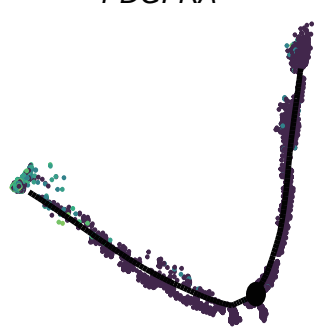

LGR5

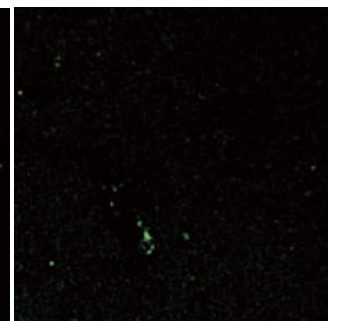

DAPI
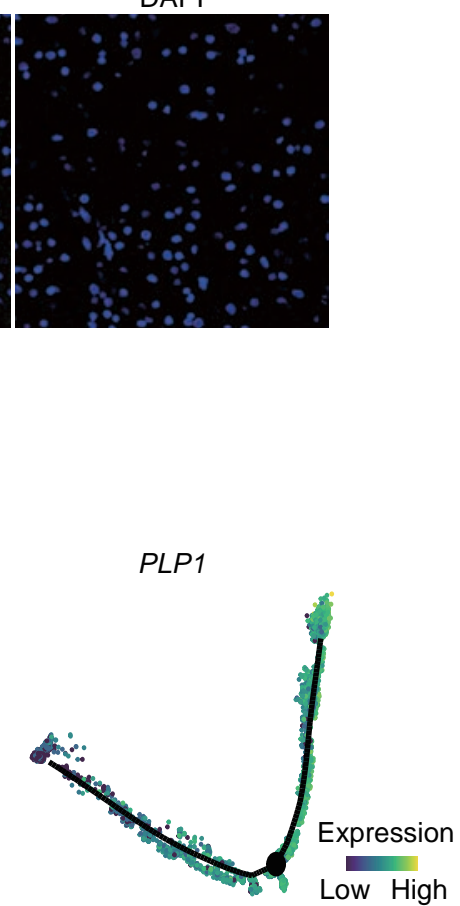
bioRxiv preprint doi: https://doi.org/10.1101/2021.12.13.472311; this version posted December 13, 2021. The copyright holder for this preprint (which was not certified by peer review) is the author/funder, who has granted bioRxiv a license to display the preprint in a

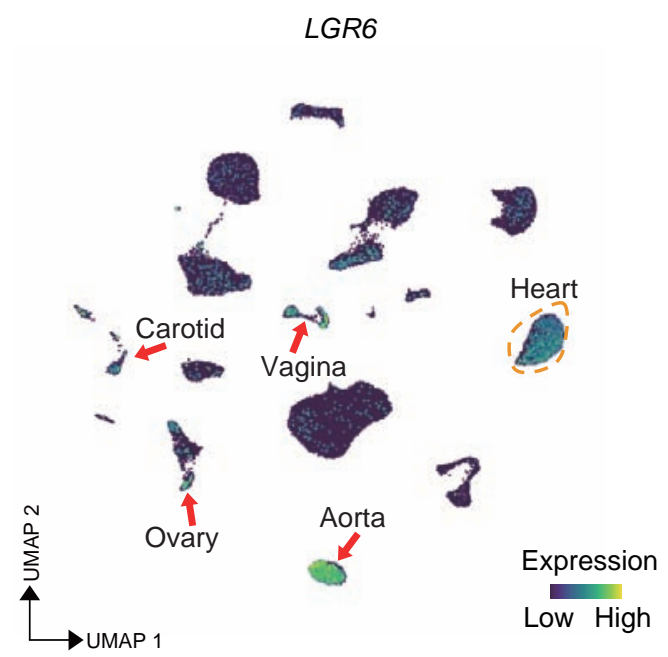

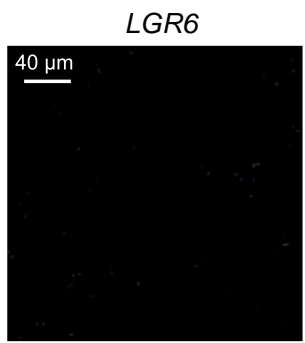

Merged

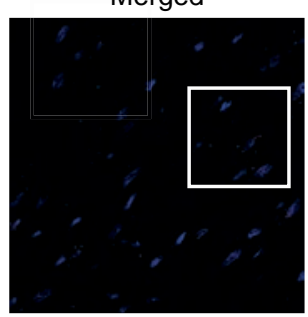

Monkey heart (5,881 nuclei)

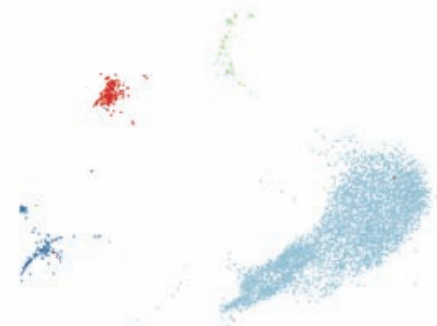

$\begin{array}{lll}\text { Cardiomyocyte } & \text { Macro } & \text { SMC Unknown } \\ \text { - Endo } & \text { Pericyte } & \text { Stromal }\end{array}$

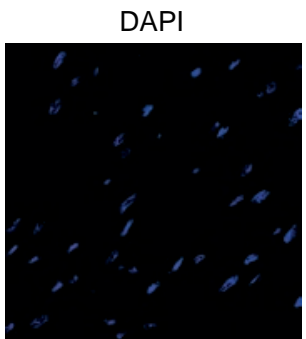

Merged (zoom)

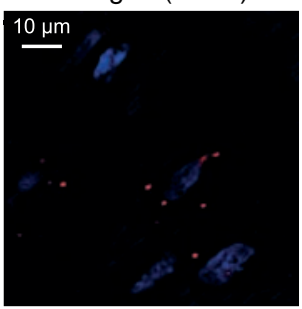

Mouse heart $(8,904$ nuclei)

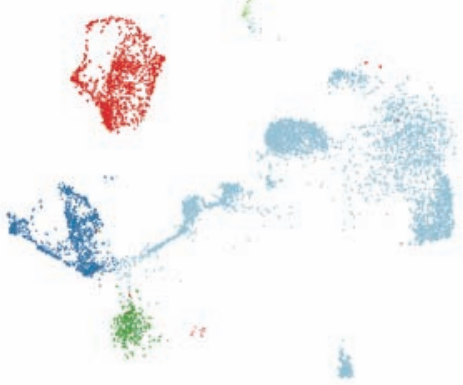

LGR6 (mouse heart)

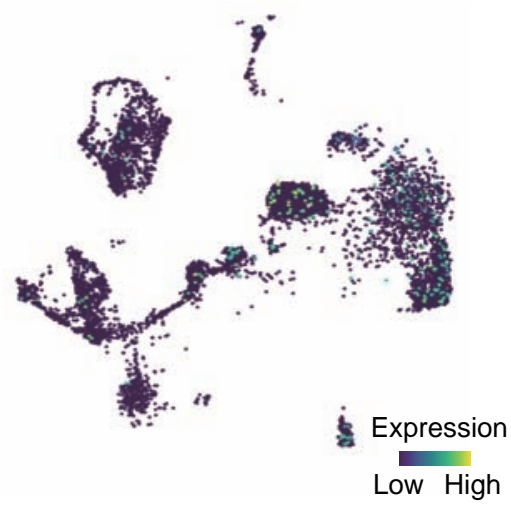

GO terms for DEG in folliculostellate

g

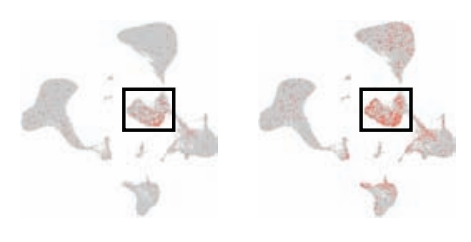

CXCR4
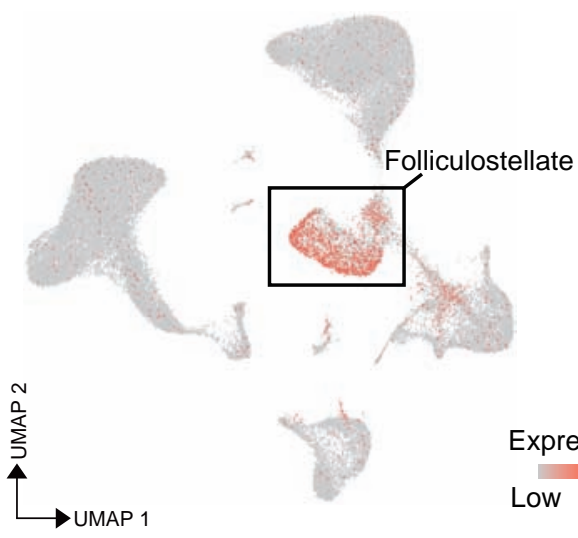

Expression

Low High
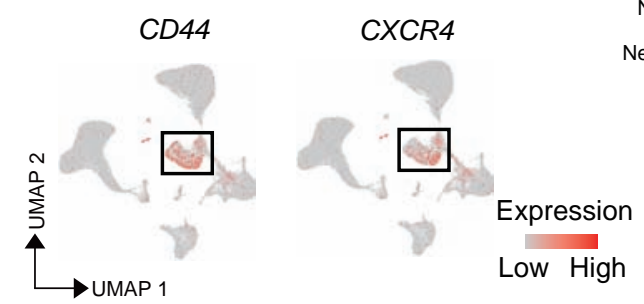
bioRxiv preprint doi: https://doi.org/10.1101/2021.12.13.472311; this version posted December 13, 2021. The copyright holder for this

preprint (which was not certified by peer review) is the author/funder, who has granted bioRxiv a license to display the preprint in

a perpetuity. It is made available:under

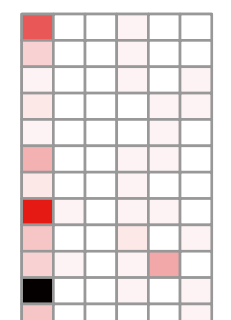

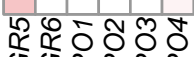

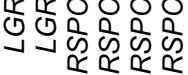

\section{$0 \quad 2.5$}

b

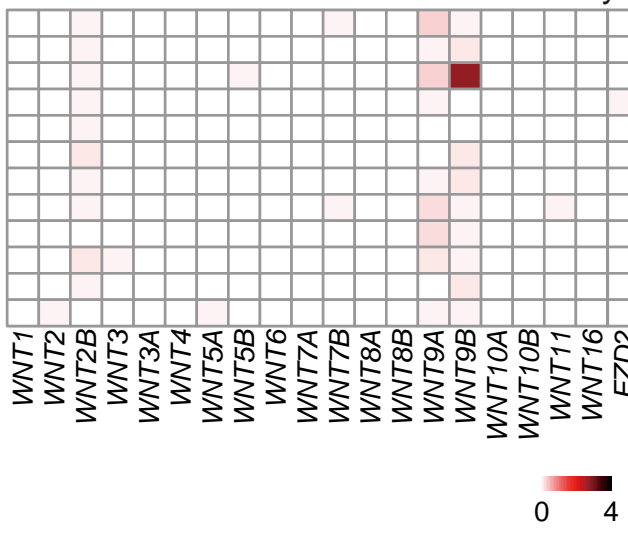

Ligand RSPO1-4 : Receptor LGR5,6

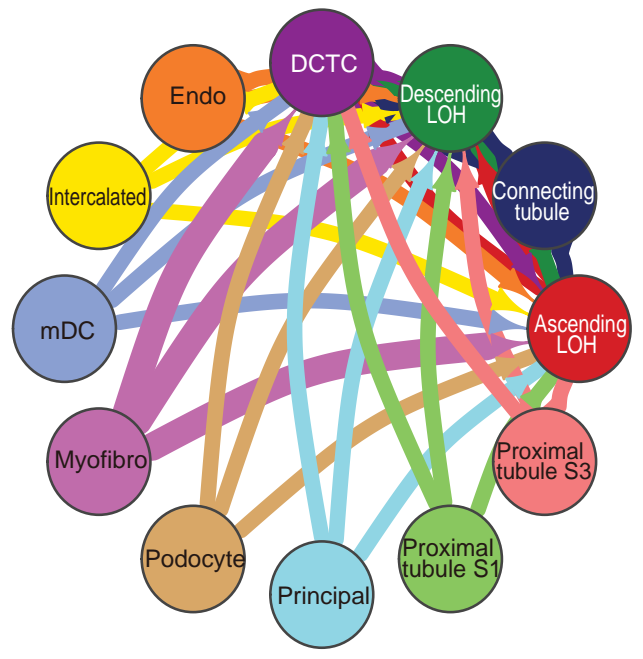

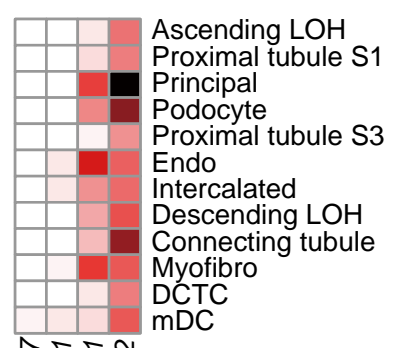

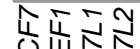

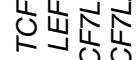

C

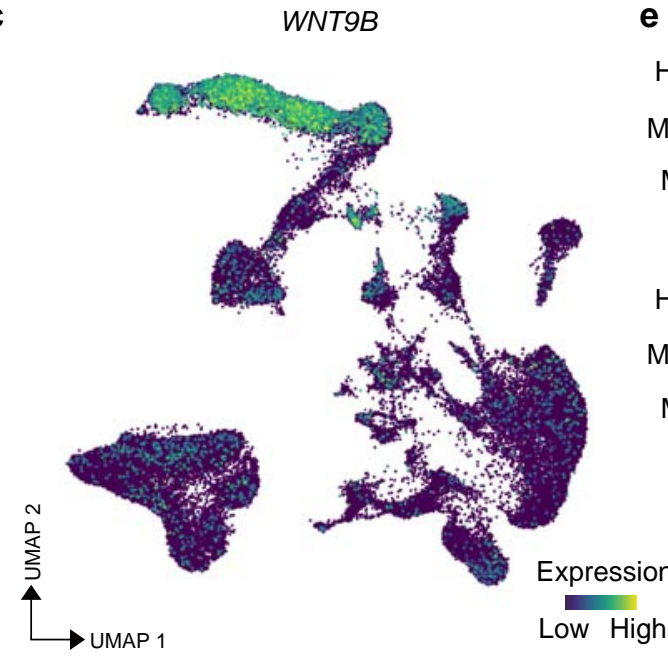

d

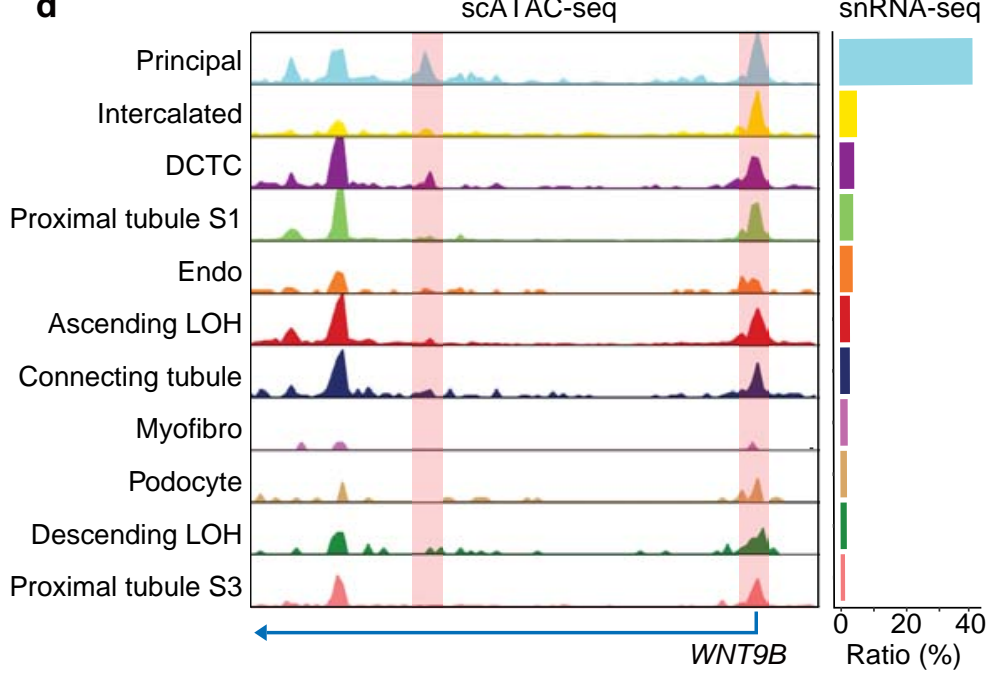

e
Principal

tercalated

ule S1

do

WNT9B
08 Expression

Ligand WNT factors : Receptor FZD etc
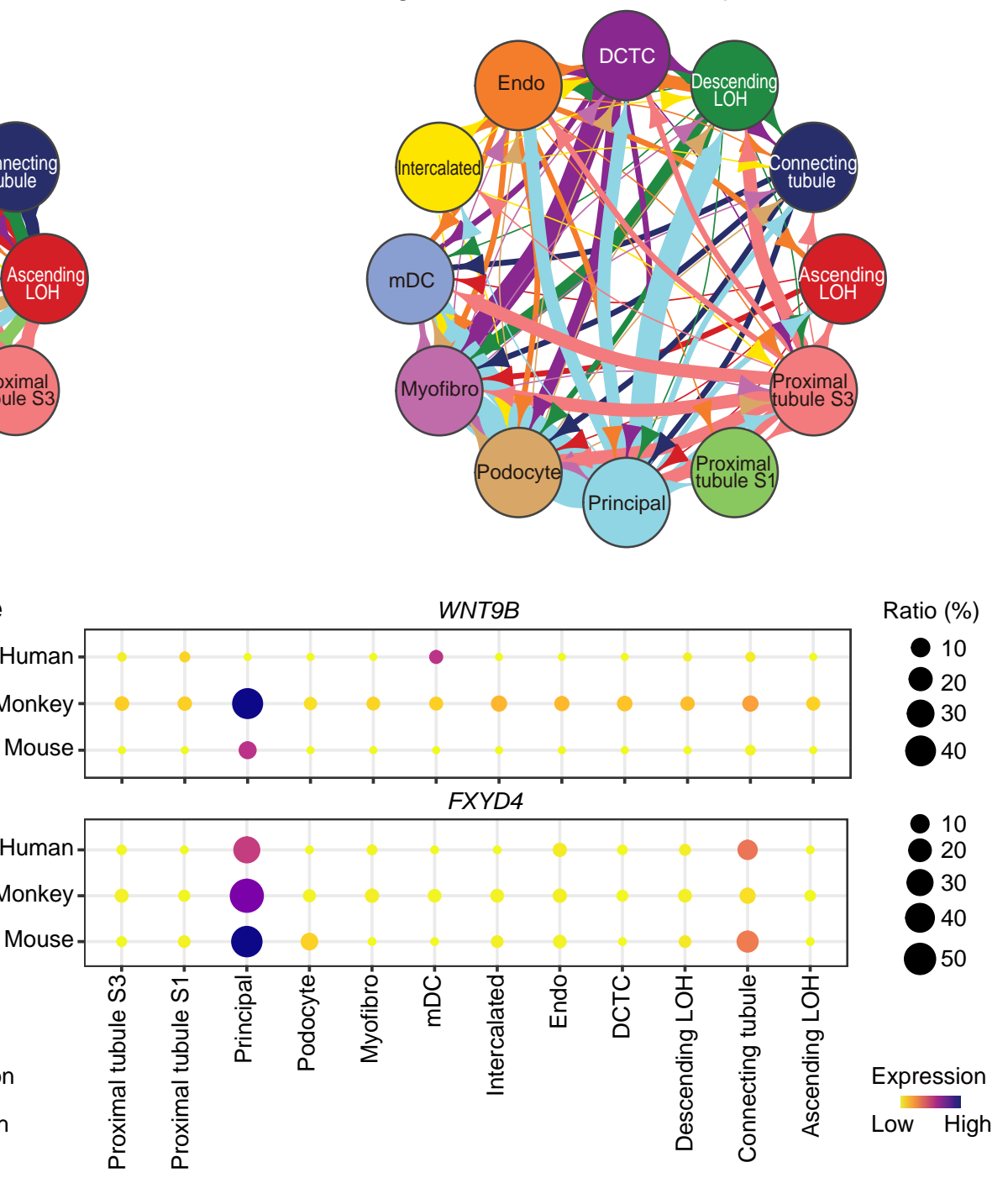

f

Proxim

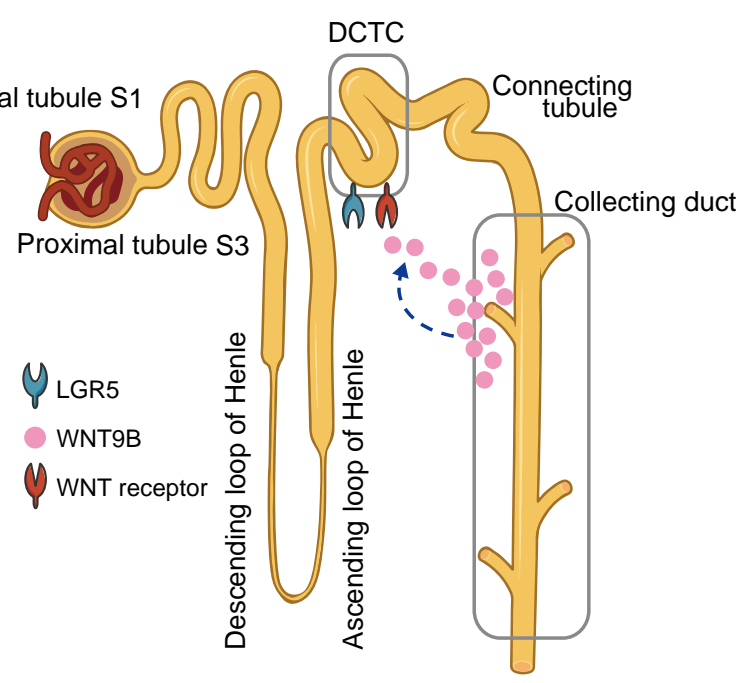


1101/2021.12.13.472311; this version posted December 13,2021 . The copyright holder for this preprint (which was not certified by peer review) is the author/funder, who has granted bioRxiv a license to display the preprint in perpetuity. It is made availabte underach-BY-NC-ND 4.0 International license.
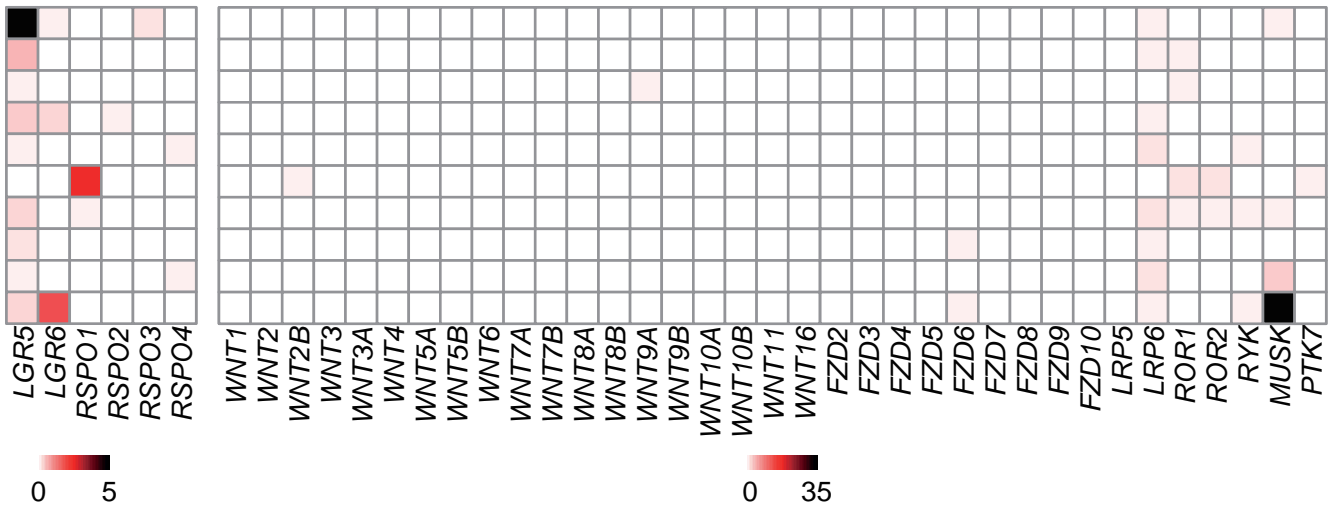

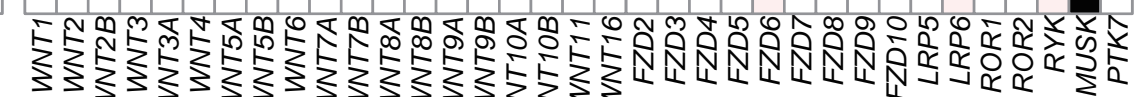

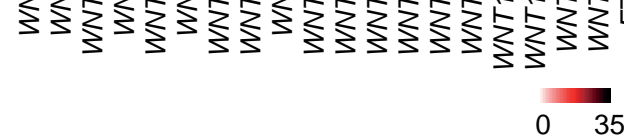

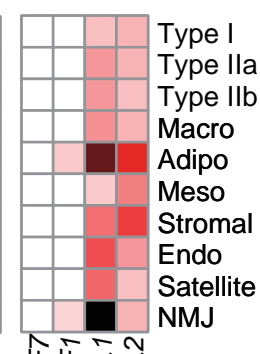

出出先

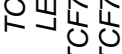

$0 \quad 10$ Expression
Ligand RSPO1-4 : Receptor LGR5,6

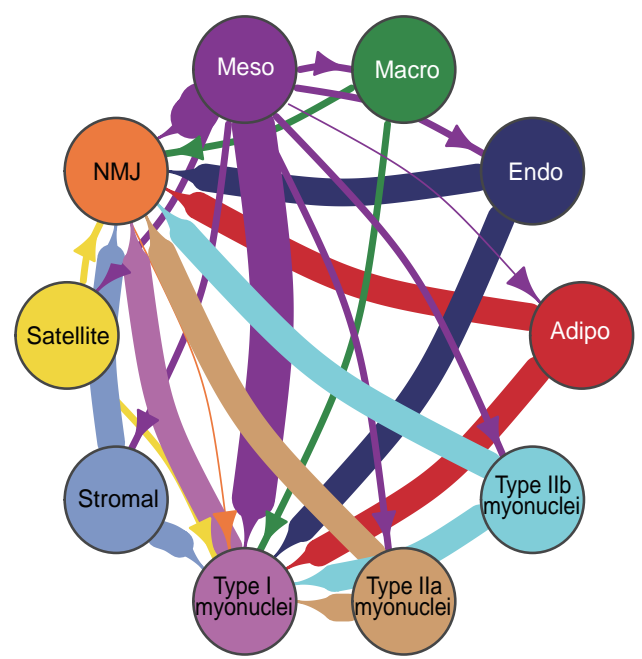

Ligand WNT factors : Receptor FZD etc

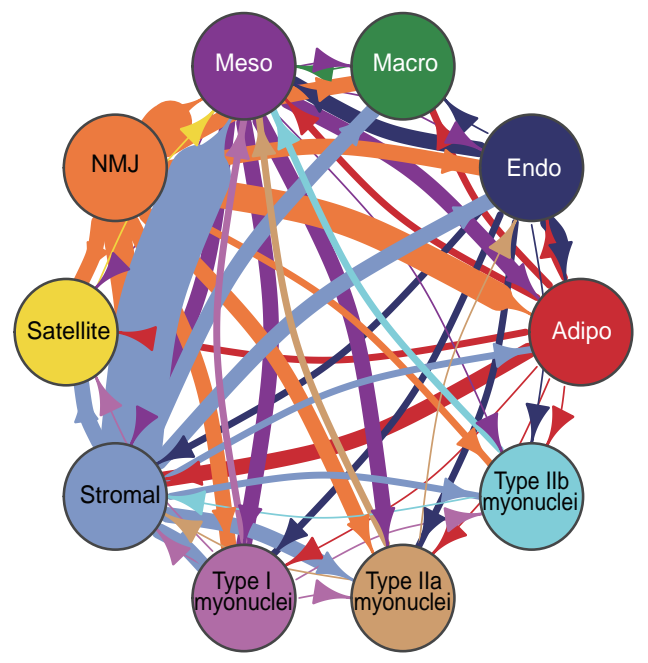

Epididymis
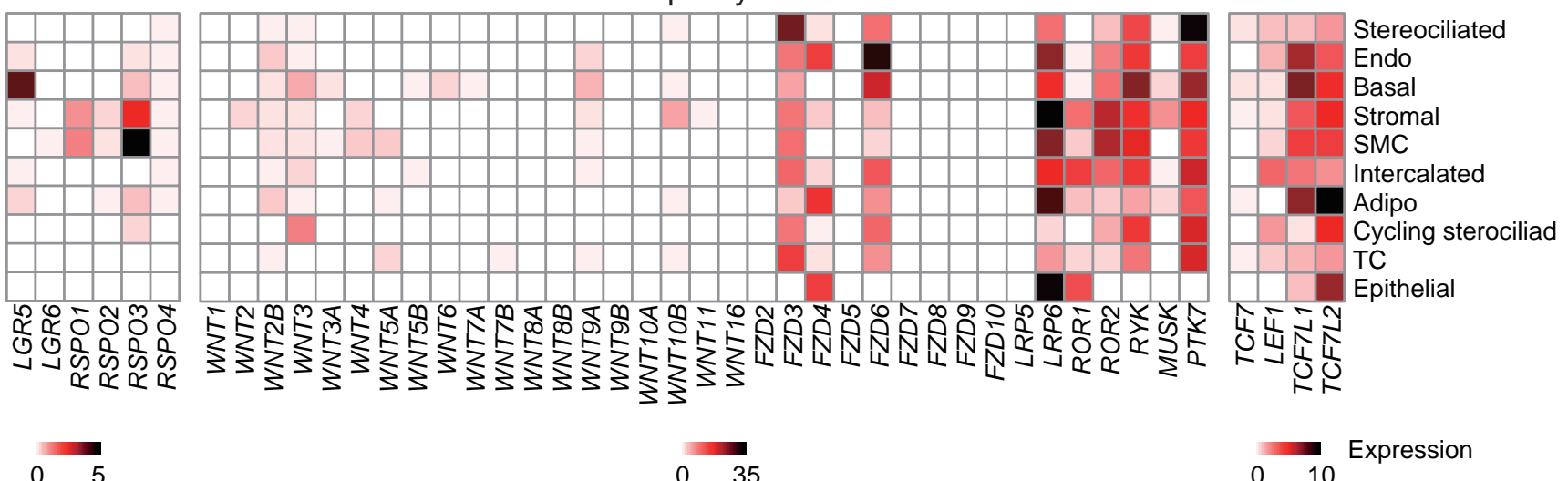

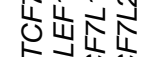

Endo

$0 \quad 10$ Expression

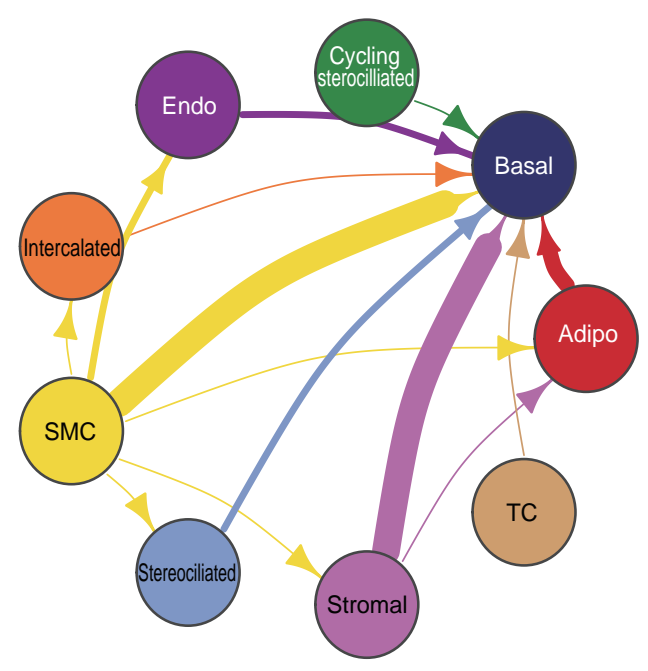

Ligand WNT factors : Receptor FZD etc

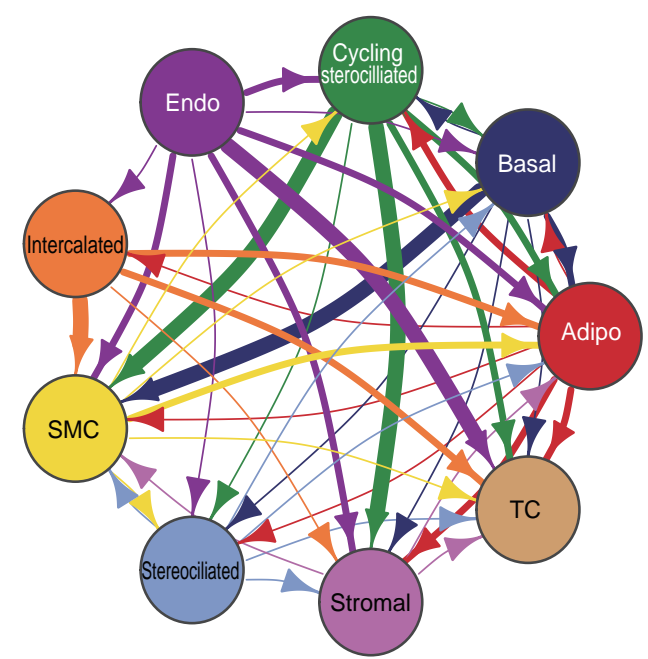


bioRxiv preprint doi: https://doi.org/10.1101/2021.12.13.472311; this version posted December 13, 2021. The copyright holder for this preprint (which was not certified by peer review) is the author/funder, who has granted bioRxiv a license to display the preprint in perpetuity. It is made avaitable $y$ hder a a 6 C-BY-NC-ND 4.0 International license.

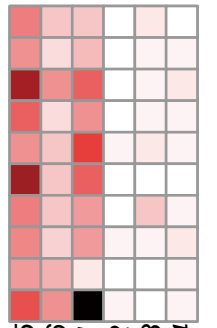

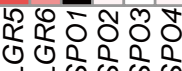

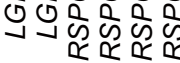

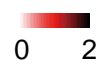

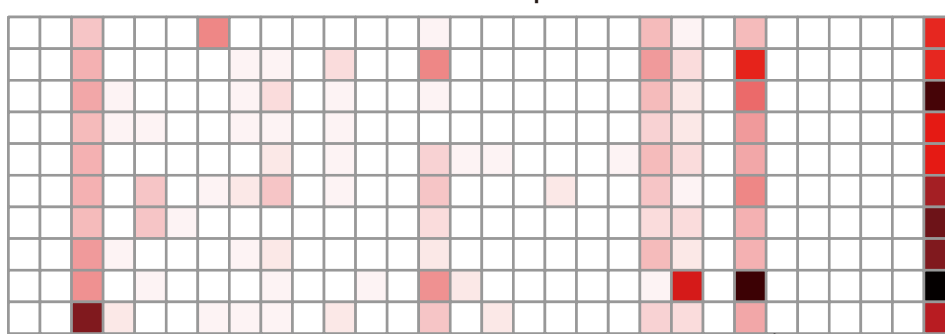

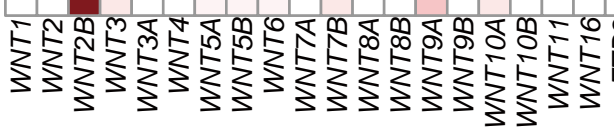

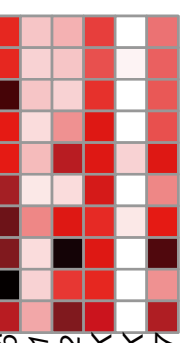

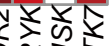

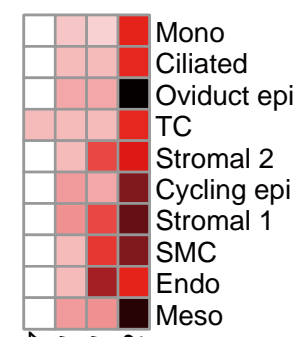

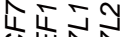

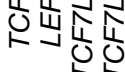

04 Expression
Ligand RSPO1-4 : Receptor LGR5,6

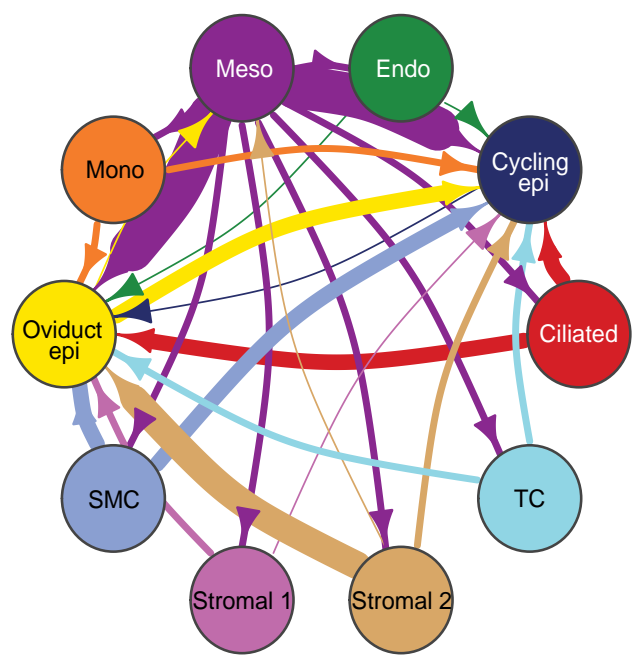

Ligand WNT factors : Receptor FZD etc

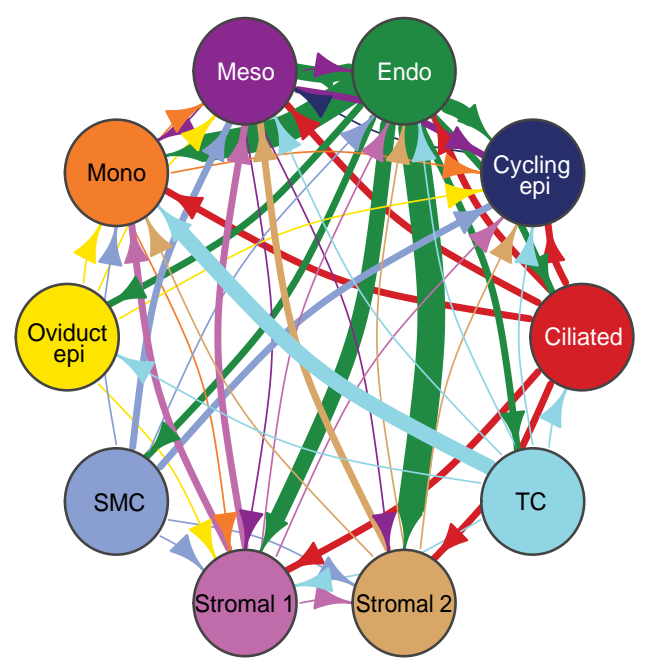

Liver

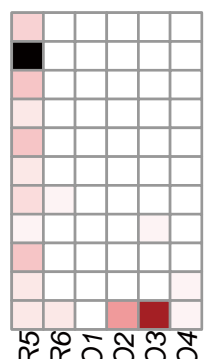

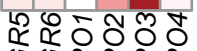

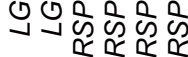

$0 \quad 3$

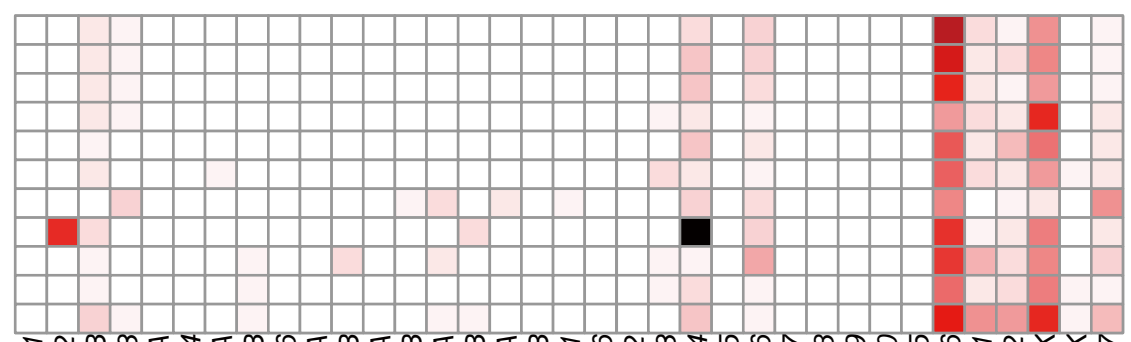

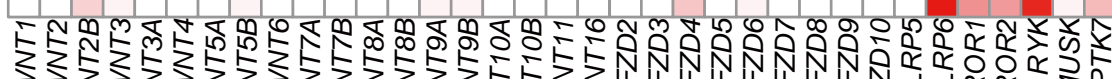

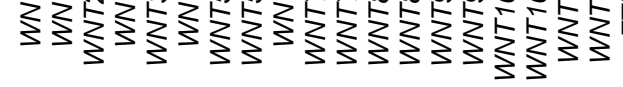

Ligand RSPO1-4 : Receptor LGR5,6

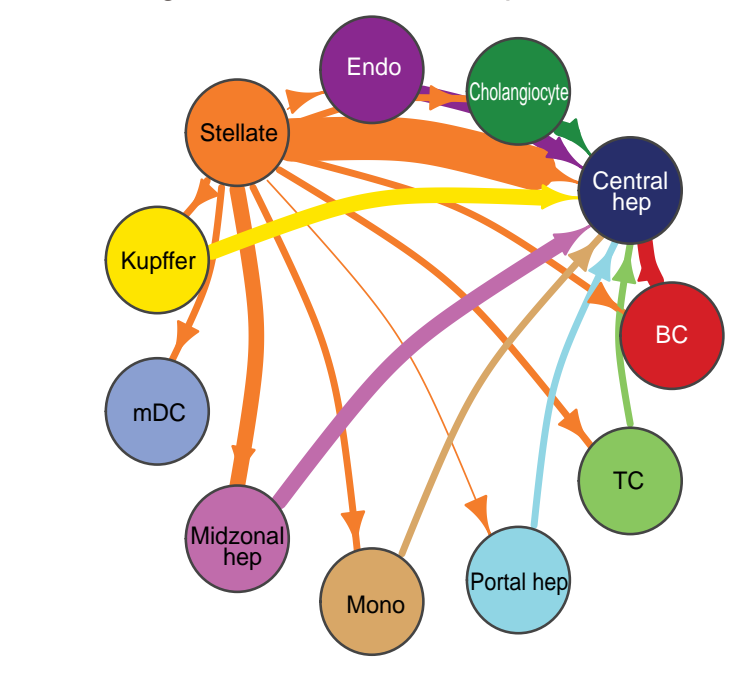

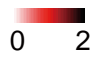

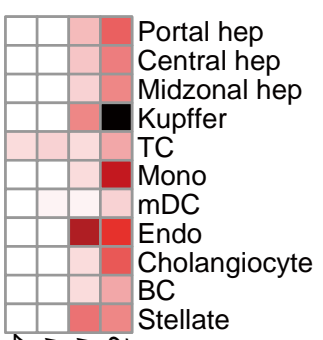

$\widehat{4} \sqrt{4}-7 \sqrt{3}$

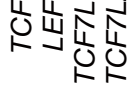

010 Expression
Ligand WNT factors : Receptor FZD etc

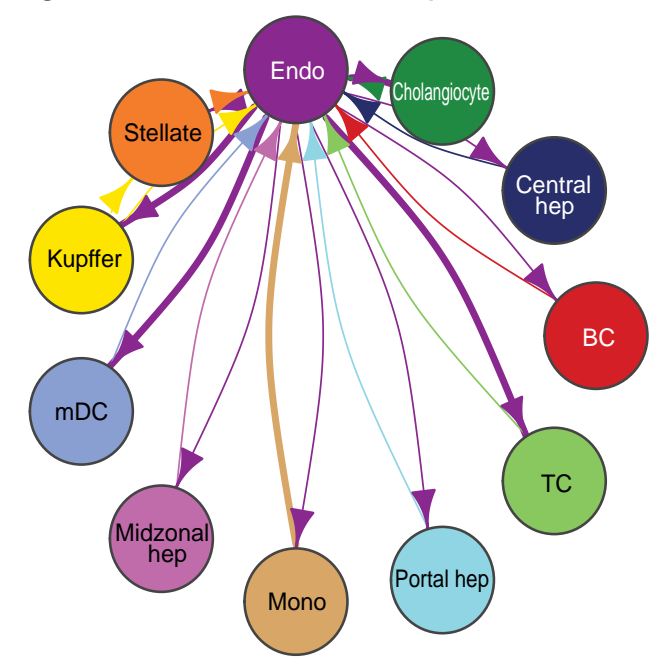


bioRxiv preprint doi: https://doi.org/10.1101/2021.12.13.472311; this version posted December 13, 2021. The copyright holder for this

preprint (which was not certified by peer review) is the author/funder, who has granted bioRxiv a license to display the preprint in perpetuity. It is made availablectuder aCC-BY-NC-ND 4.0 International license.

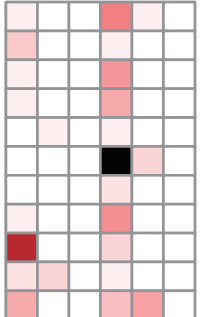

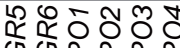

9
$y$

$0 \quad 2.5$

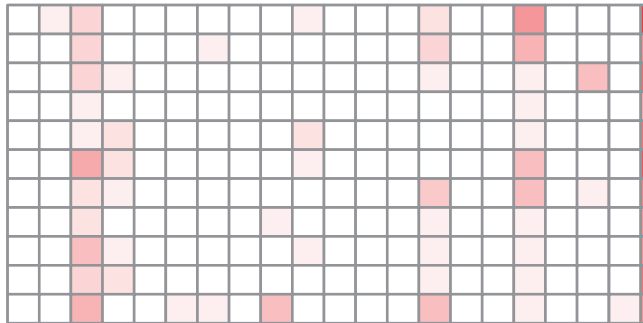

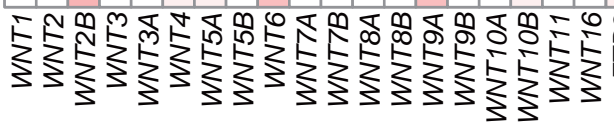

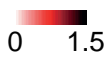

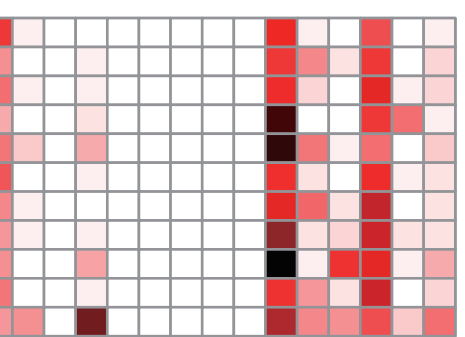

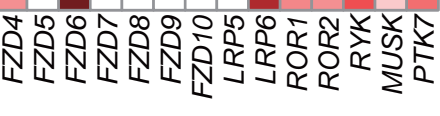

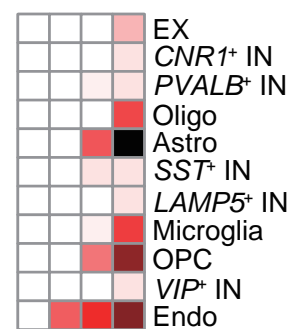

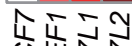

虬出곤

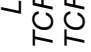

03

Expression
Ligand RSPO1-4 : Receptor LGR5,6

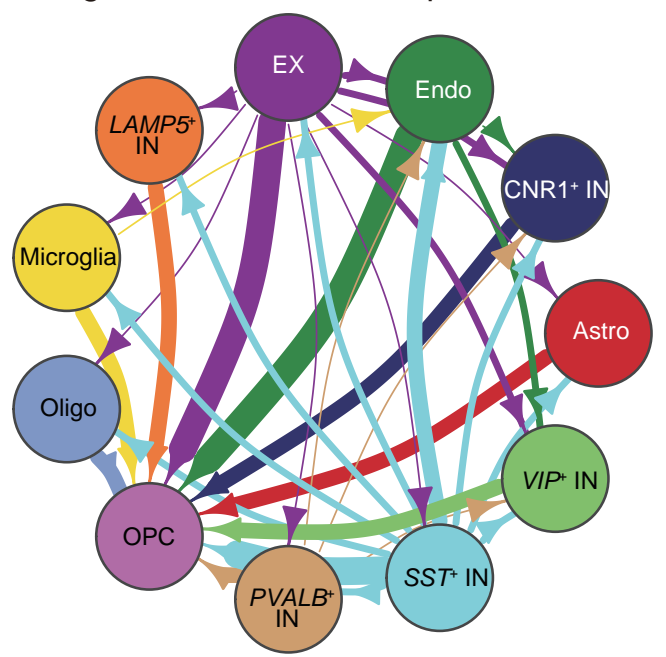

Ligand WNT factors : Receptor FZD etc

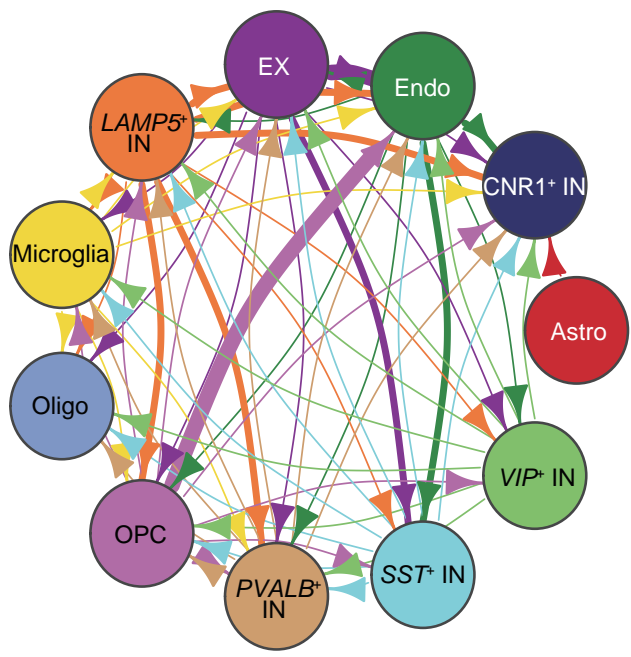

Ovary

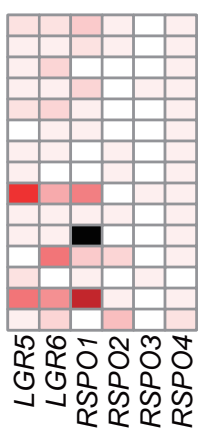

02

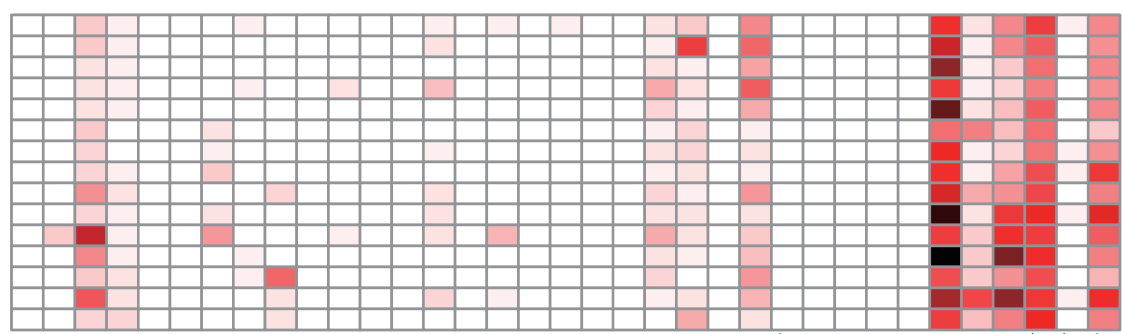

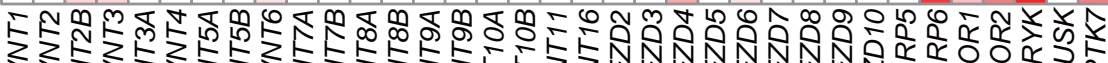

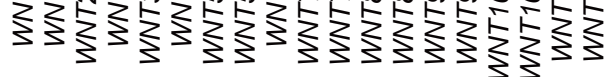

Ligand RSPO1-4 : Receptor LGR5,6

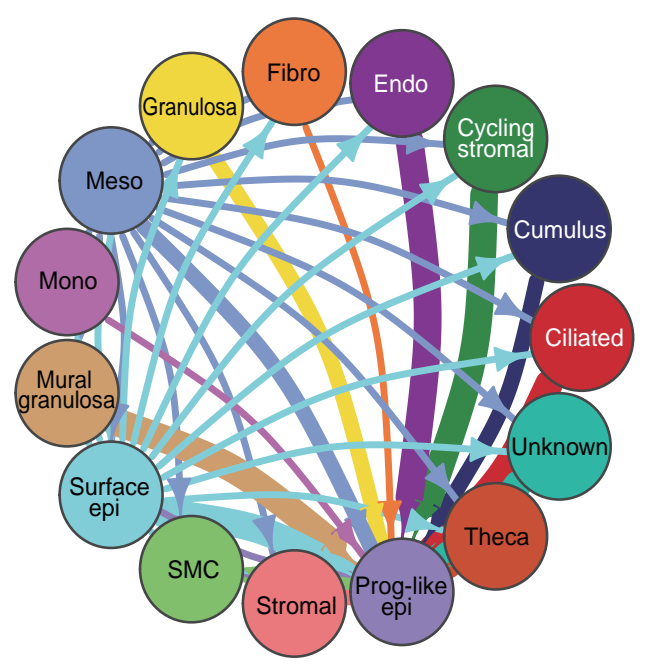

Ligand WNT factors : Receptor FZD etc

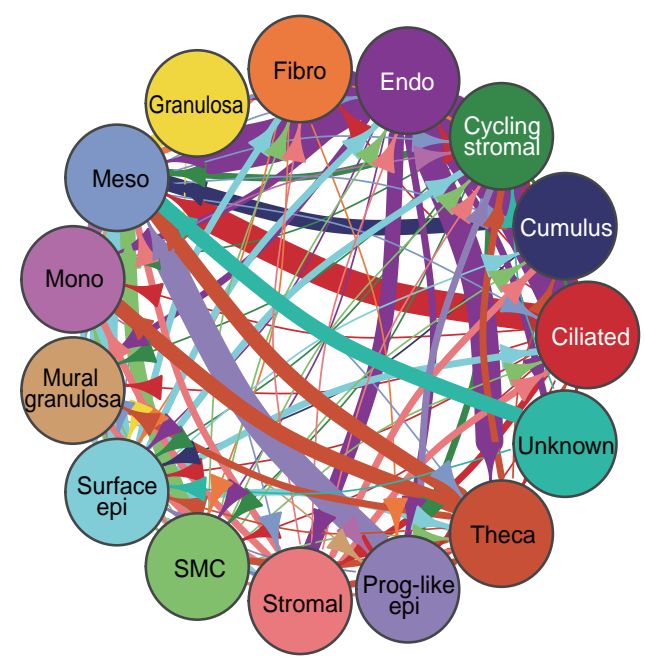


bioRxiv preprint doi: https://doi.org/10.1101/2021.12 13.472311; this version posted December 13, 2021. The copyright holder for this

preprint (which was not certified by peer review) is the author/funder, who has granted bioRxiv a license to display the preprint in perpetuity. It is made available under aCC-BY-NC-ND 4.0 International license.

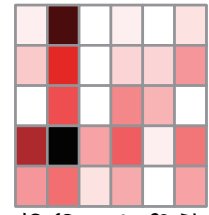

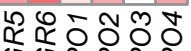

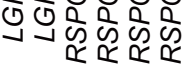

0.7

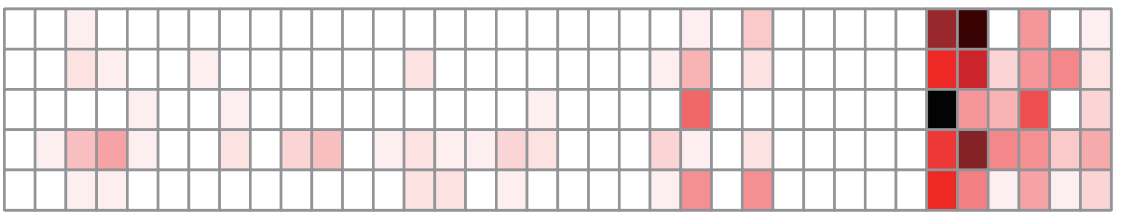

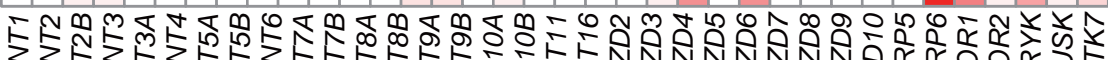

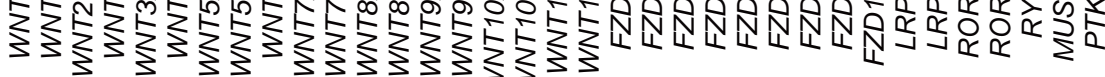

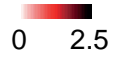

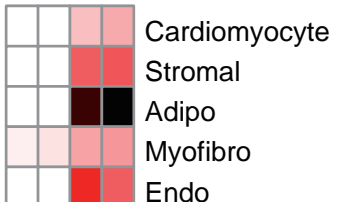

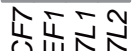

\section{Endo}

Ligand RSPO1-4 : Receptor LGR5,6

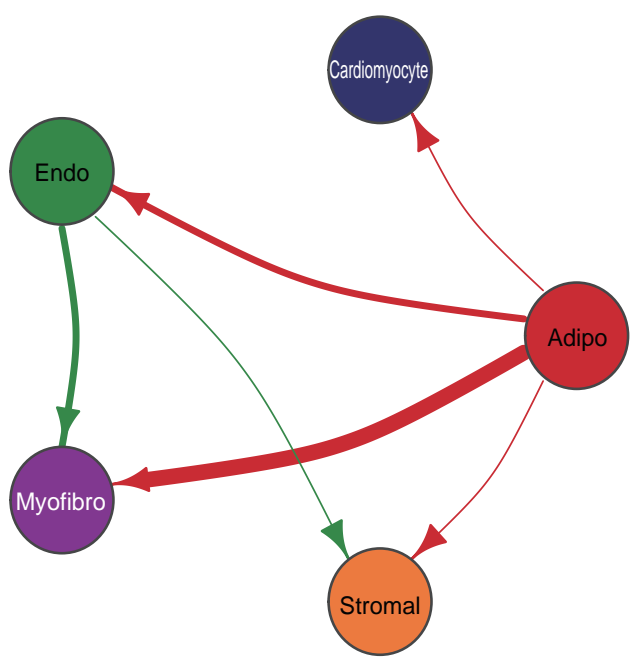

Ligand WNT factors : Receptor FZD etc

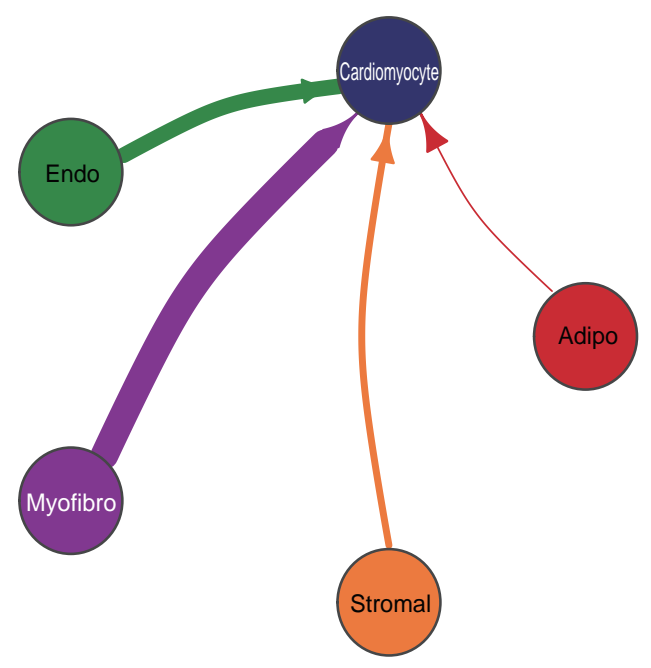

Pituitary gland

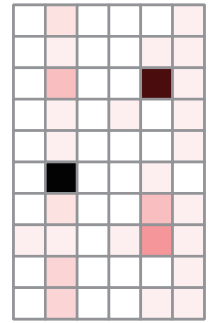

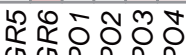

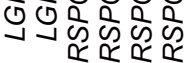

$0 \quad 2.5$

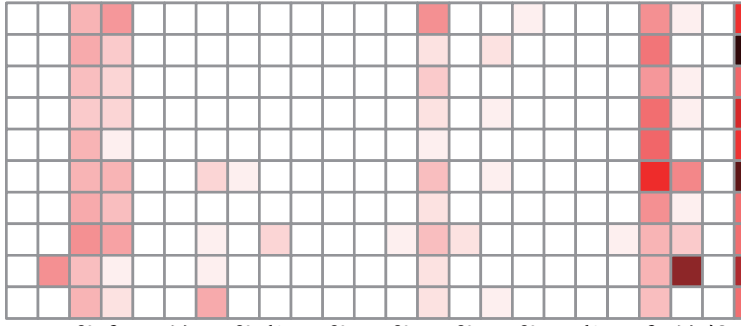

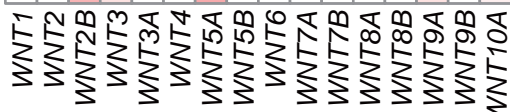

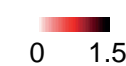

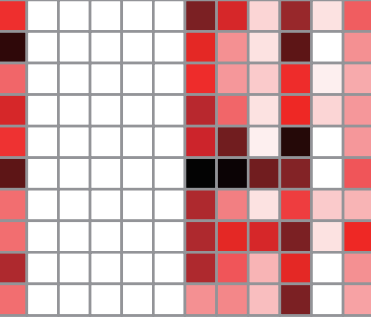

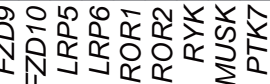

$$
\text { 年 }
$$

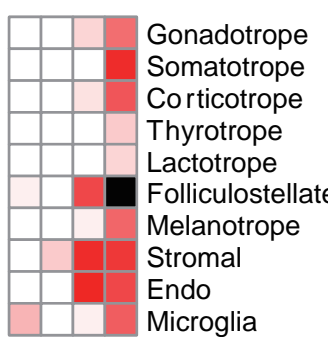

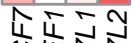

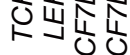

U난

04 Expression
Ligand RSPO1-4 : Receptor LGR5,6

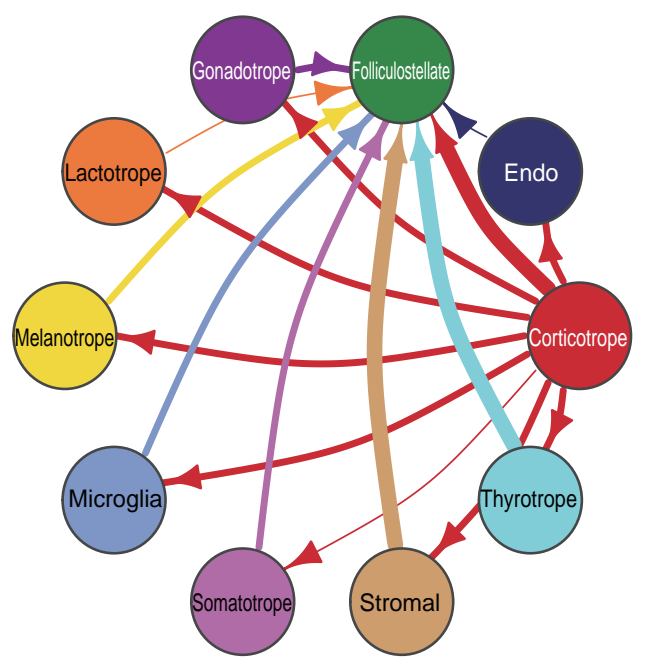

Ligand WNT factors : Receptor FZD etc

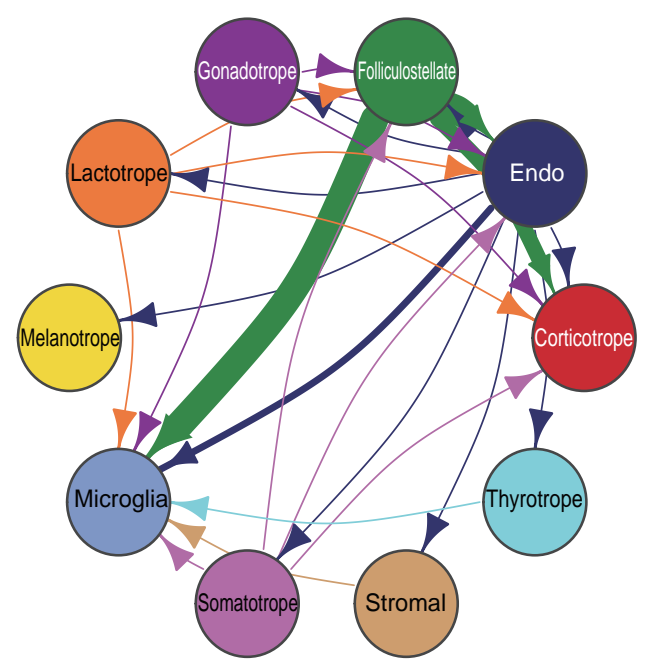


bioRxiv preprint doi: https://doi.org/10.1101/2021.12.13.472311; this version posted December 13, 2021. The copyright holder for this preprint (which was not certified by peer review) is the author/funder, who has granted bioRxiv a license to disglasy.ther preprint in

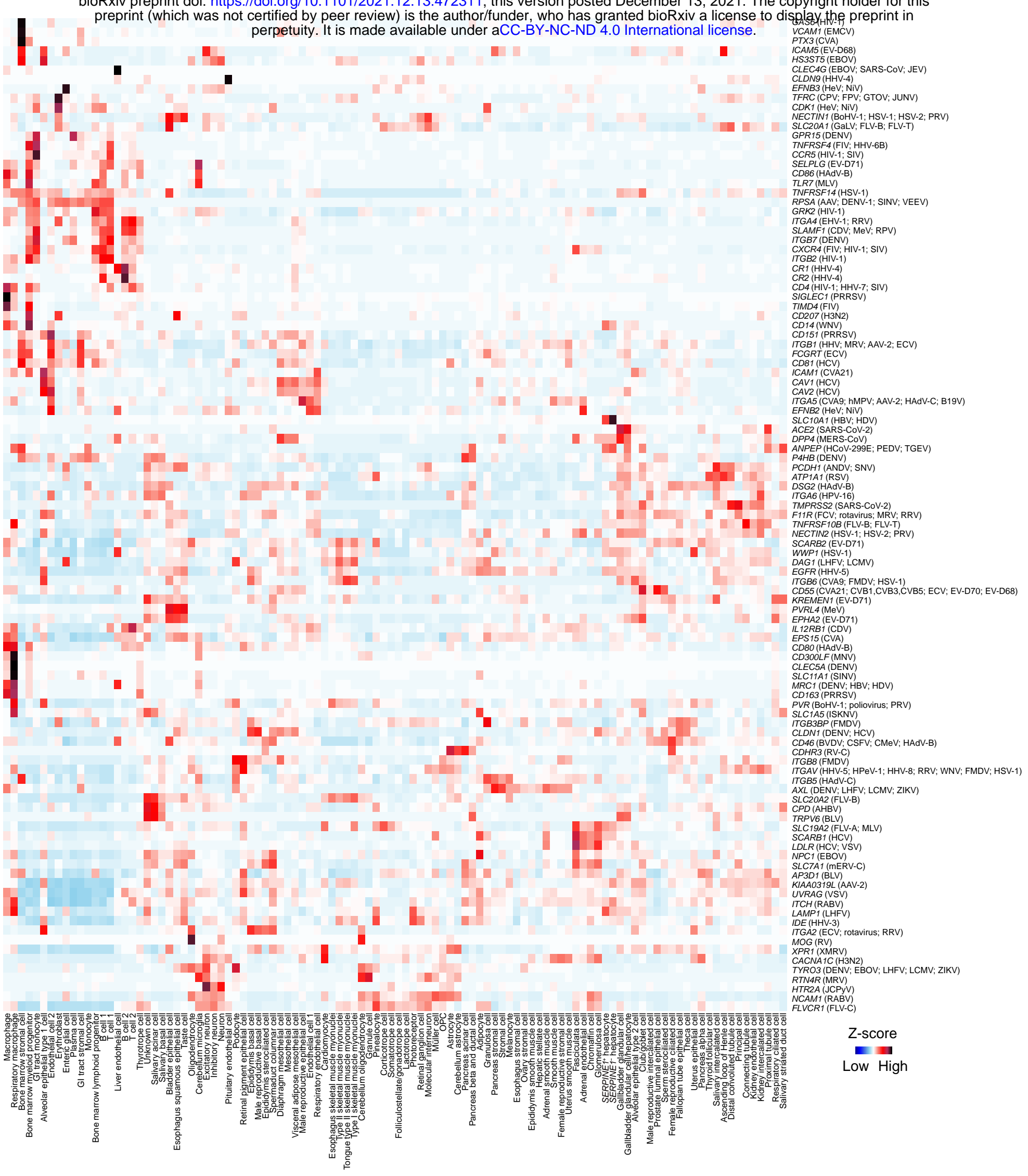


bioRxiv preprint doi: https://doi.org/10.1101/2021.12.13.472311; this version posted December 13, 2021. The copyright holder for this

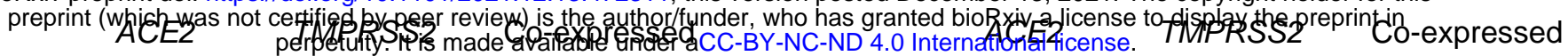

Abdominal wall Epididymis

Adrenal gland Esophagus

Aorta

Fallopian tube

Bone marrow

Bladder

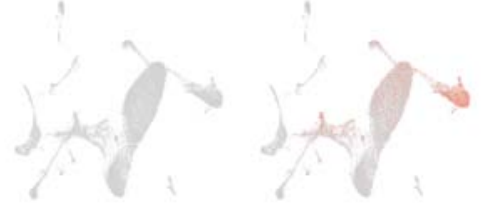

Gallbladder

Heart

Bronchus

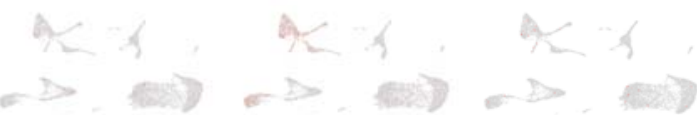

Cerebellum

Carotid

Colon
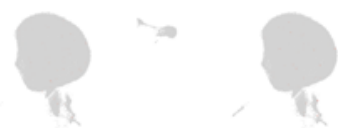

Kidney
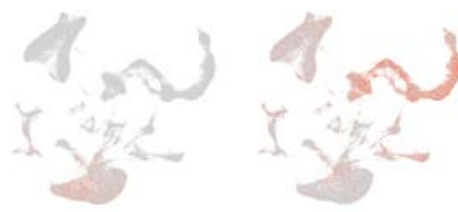

Liver
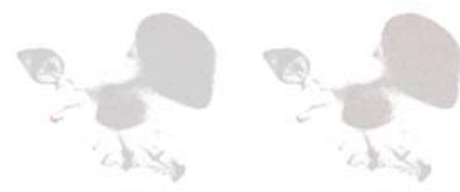

Lung

Lymph node

Neocortex

Diaphragm

Expression

Low High

Ovary

Duodenum

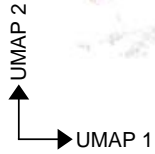


Pancreas

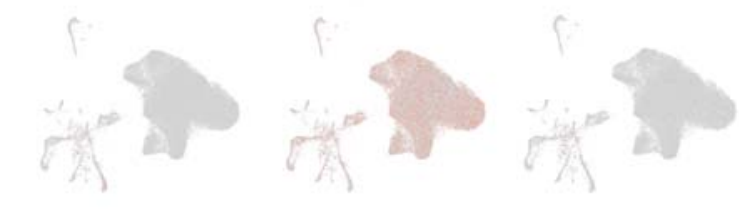

Stomach

PBMC

Pigmentary epithelium

Subcutaneous adipose

Pineal gland

Substantia nigra

Thyroid

Pituitary gland
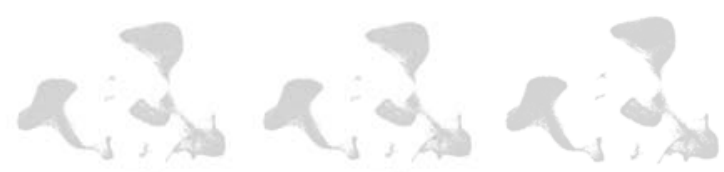

Tongue

Prostate

Retina

Trachea

Salivary gland
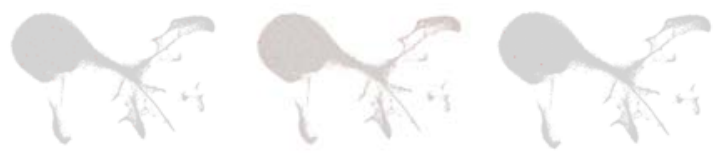

Tonsil

Spermaduct

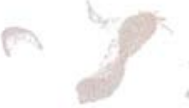

P 8

Vagina

Uterus

Spinal cord

Visceral adipose 
bioRxiv preprint doi: https://doi.org/10.1101/2021.12.13.472311; this version posted December 13,2021 . The copyright holder for this
preprint (which was not certified by peer review) is the author/funder, who has granted bioRxiv a license to display the preprint in

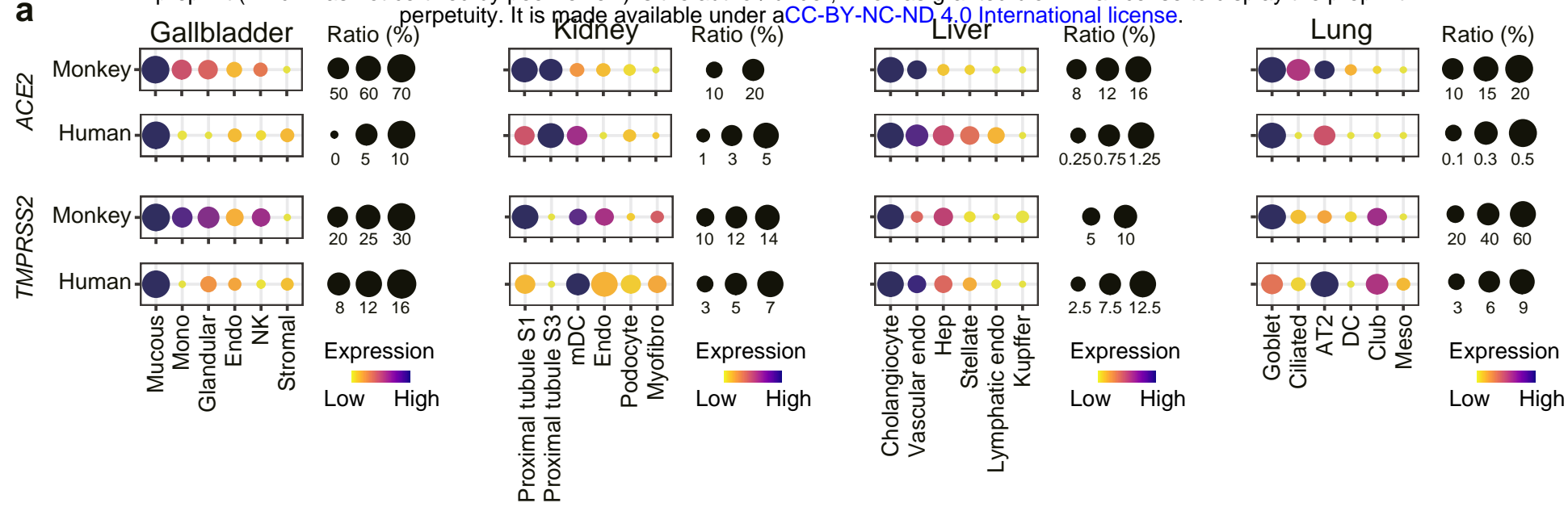

b

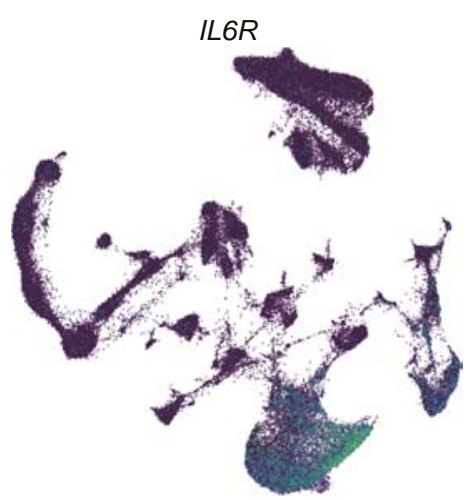

$A C E 2^{+} I L 6 R^{+}$

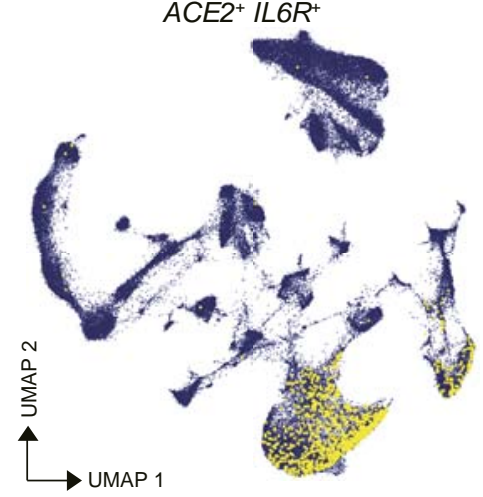

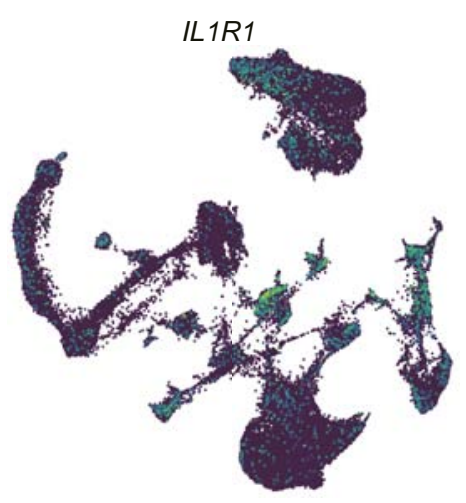

ACE2 $^{+} I L 1 R 1^{+}$

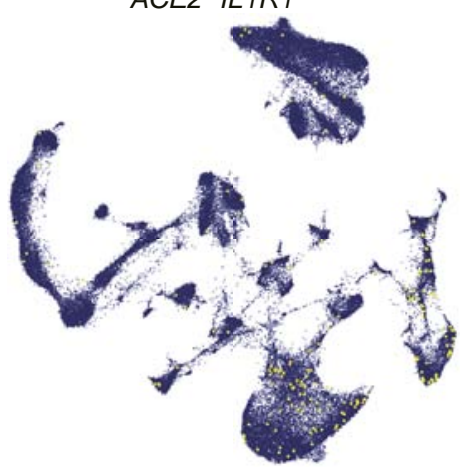

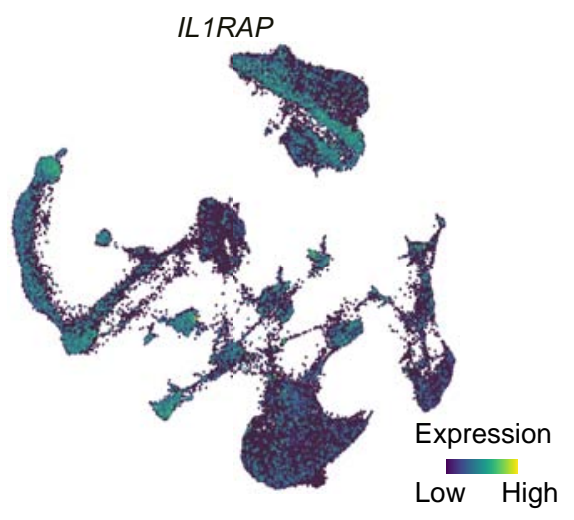

ACE2 $^{+}$ILIRAP ${ }^{+}$

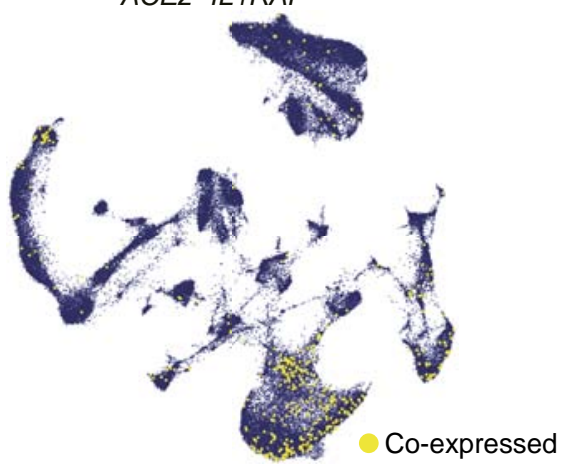

C

\begin{tabular}{|ll|}
\hline$\prod_{\text {ACE2 }}$ & $Y_{\text {ILGR }}$ \\
$Y_{\text {TMPRSS2 }}$ & IL6 \\
& ILARS-CoV-2 \\
\hline
\end{tabular}

Macrophages
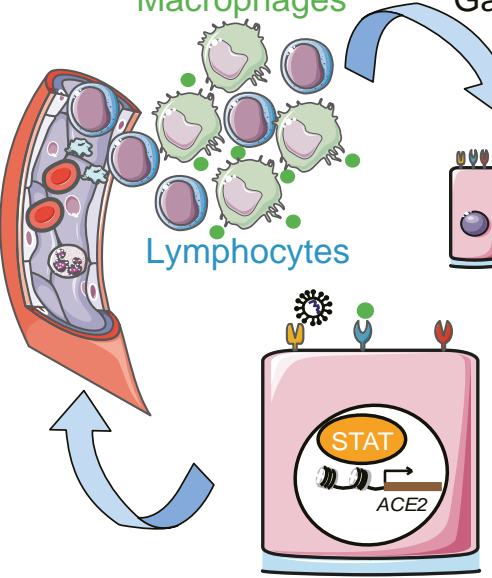

萧 Lung 漟

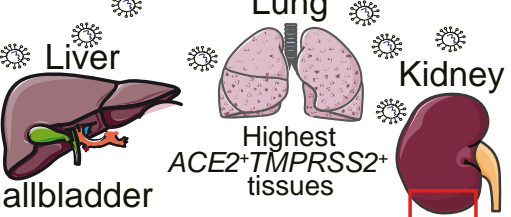

Gallbladder
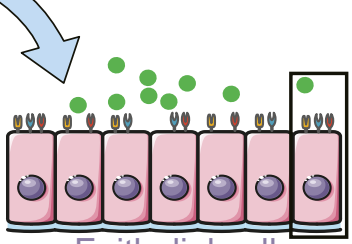

Epithelial cells

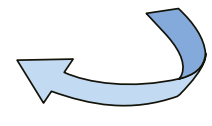

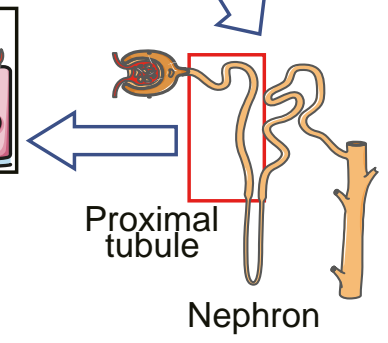

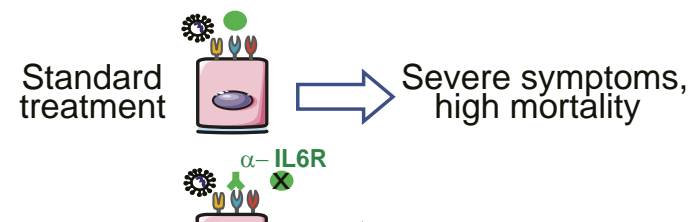

Tocilizumab $\square$ Manageable disease

Nephron

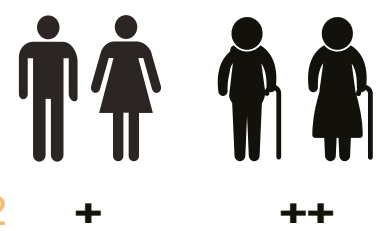

$+$

$++$ 
bioRxiv preprint doi: https://doi.org/10.1101/2021.12.13.472311; this version posted December 13, 2021. The copyright holder for this preprint (which was not certified by peer review) is the author/funder, who has granted bioRxiv a license to display the preprint in

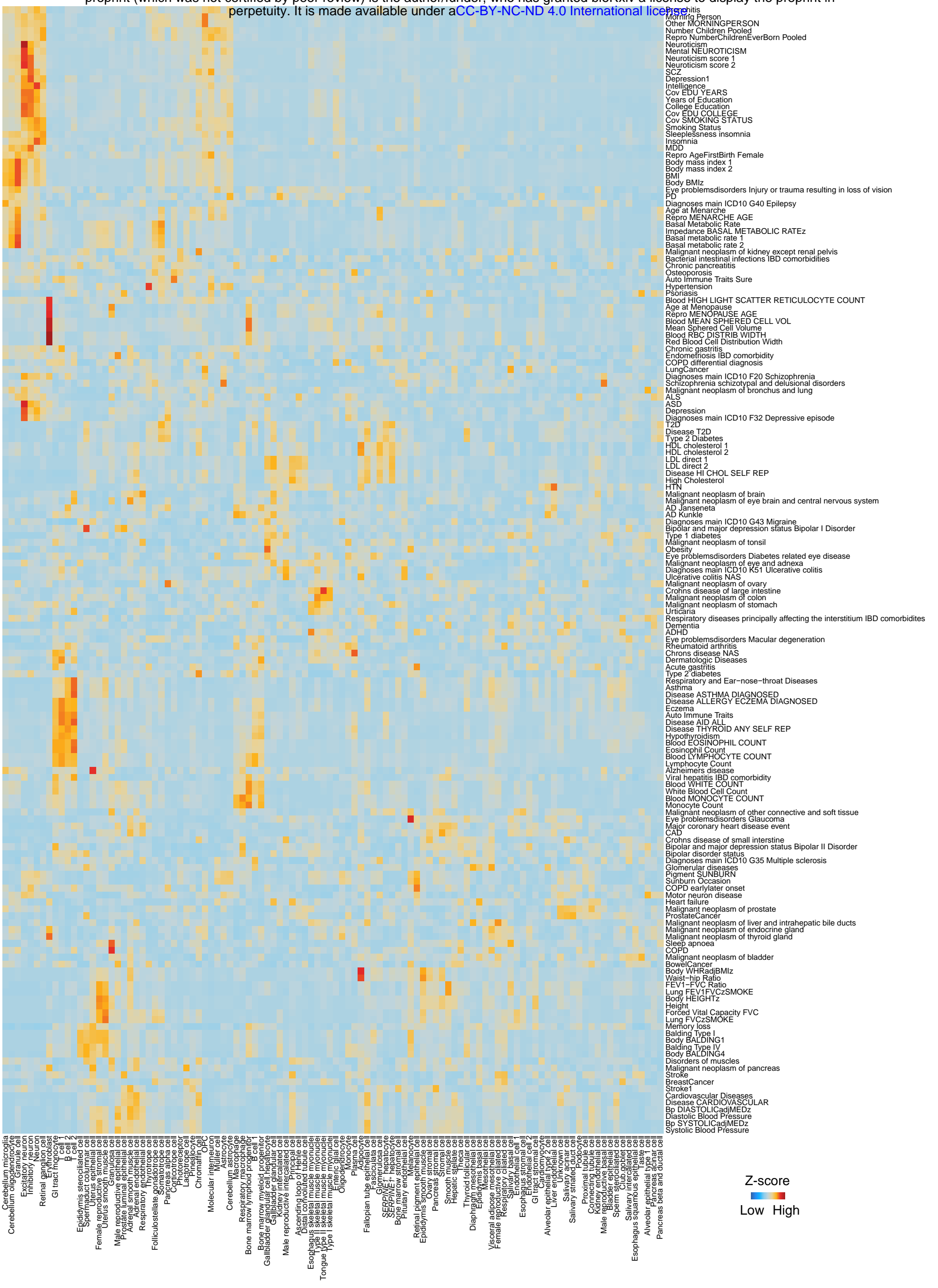


bioRxiv preprint doi: https://doi.org/10.1101/2021.12.13.472311; this version posted December 13, 2021. The copyright holder for this preprint (which was not certified by peer review) is the author/funder, who has granted bioRxiv a license to display the preprint in

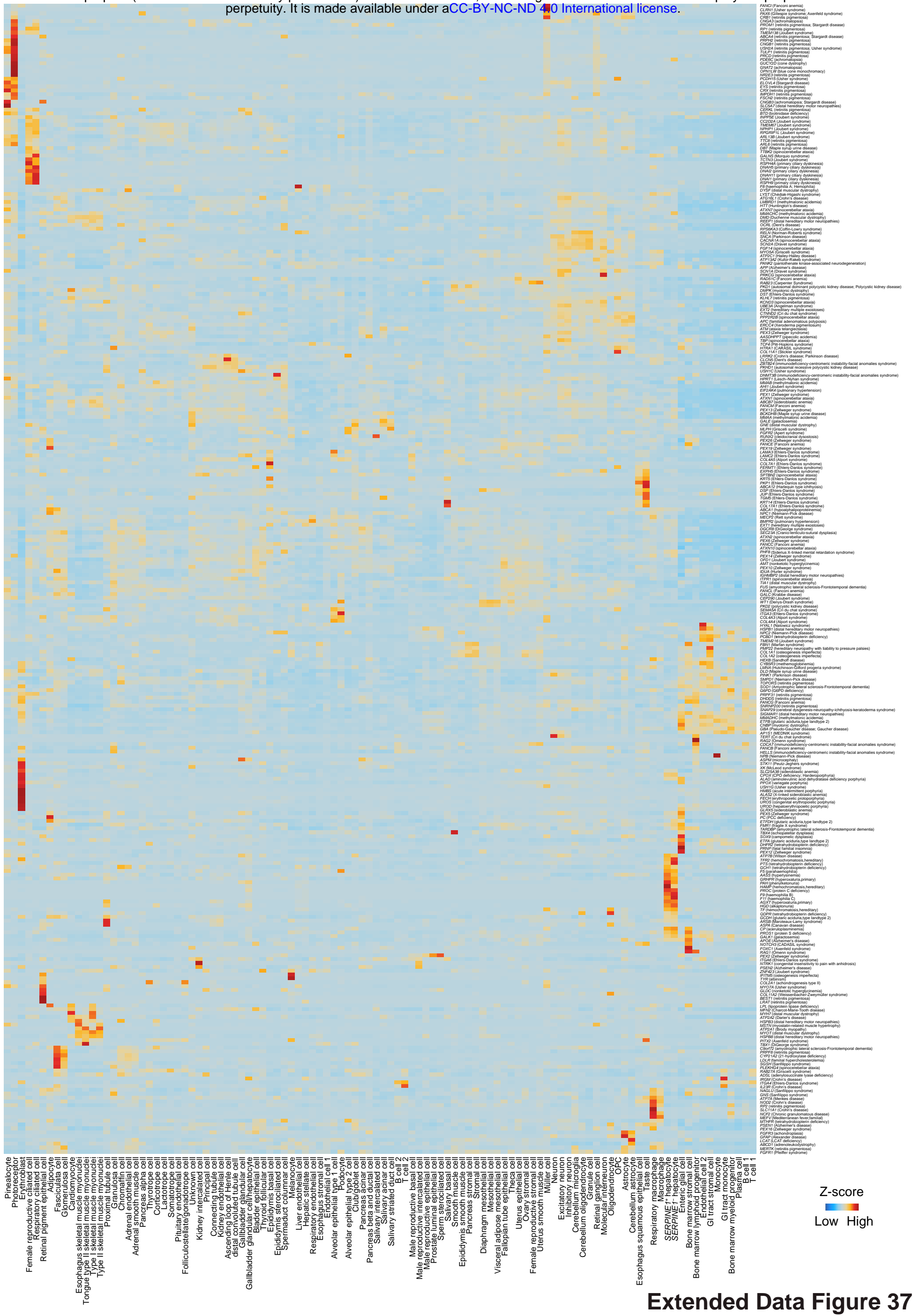

